\title{
Does non-suicidal self-injury function primarily as an experientially avoidant behaviour within Aotearoa New Zealand?
}

Robyn Lisa Langlands

\author{
A thesis submitted to Victoria University of Wellington \\ in fulfilment of the requirements for the degree of \\ Doctor of Philosophy in Psychology
}

Victoria University of Wellington 

Theoretical, empirical, and experiential attempts at disentangling the functions of Non-Suicidal Self-Injury (NSSI) have been driven by the desire to answer the complex question: Why do people engage in self-injurious behaviours? A recently developed behavioural model of NSSI - the Experiential Avoidance Model (EAM; Chapman, Gratz, \& Brown, 2006) - proposes that self-injury functions primarily as a form of negatively reinforced, experiential avoidance and places particular emphasis on emotional avoidance. Experiential avoidance is conceptualised as a behavioural process whereby people are unwilling to tolerate distressing emotions, thoughts, memories, or physical sensations and engage in behaviours to change, avoid, or escape from these aversive, intrapersonal experiences (Hayes, Wilson, Gifford, Follette, \& Strosahl, 1996).

Although the results of international studies support the key assumptions of this model to varying degrees (Klonsky, 2007; Klonsky \& Glenn, 2008; Nock \& Prinstein, 2004), the EAM has never been empirically evaluated within Aotearoa New Zealand. To determine whether experiential avoidance is the primary function of NSSI for people living within Aotearoa New Zealand, I designed and conducted three studies. For my first study, I interviewed 24 people who had engaged in nonsuicidal self-injurious behaviours in the previous 12 months about the antecedents and consequences of their most recent episode of self-injury. The interviews were analysed using a framework based on behavioural principles, which I developed for the purpose of this research. This method of analysis, which I called Interpretative Functional Analysis, allowed me to identify, and then compare, the functions served by discrete self-injurious episodes. Results supported the EAM (Chapman et al., 2006) in that self-injury episodes functioned predominantly as attempts to avoid or escape from intense, negative emotional experiences. Cognitive avoidance, however, also played a significant role in the self-injury trajectory, which highlighted the importance of investigating unwanted thoughts in subsequent studies. 
The second study involved surveying 198 people across Aotearoa New Zealand who had self-injured in the previous 12 months to further test whether the key assumptions of the EAM (Chapman et al., 2006) apply to a New Zealand-based population. Quantitative findings supported the model and were consistent with extant international studies in that experientially avoidant, intrapersonal functions (i.e., affect regulation and self-punishment) were identified as primary to the reinforcement and maintenance of NSSI. Intrapersonal functions, in general, were more highly endorsed than interpersonal functions. Finally, both negative affect and cognitions decreased following episodes of self-injury, while joviality increased. The increase in positive emotions undermines the EAM's (Chapman et al., 2006) exclusive focus on negative reinforcement, suggesting that positive reinforcement also has an important role to play in the continued use of NSSI.

Analyses of the open-ended, survey responses highlighted the impact of particular contextual factors (such as interpersonal conflict and community norms) on the incidence and maintenance of NSSI. Conducting a thematic analysis of the consequences of people's most recent episode of NSSI allowed me to identify two distinct themes within this data corpus. Specifically, through self-injury participants assumed two paradoxical roles, that of transgressor and helper.

For my final study, I surveyed university students across two time-points (Time $1 N=408$, Time $2 N=224$ ) about their general intrapersonal experiences (i.e., emotions and thoughts) and dispositional coping styles (e.g., global experiential avoidance, thought suppression). Negative intrapersonal experiences and avoidant coping styles were found to vary as a function of NSSI history and recency. Negative automatic thoughts and guilt at Time 1 also predicted new episodes of self-injury at Time 2. Additionally, thought suppression, not global experiential avoidance, was identified as a partial mediator of Time 1 relationships between negative intrapersonal experiences and NSSI. To conclude, the findings from this thesis are situated within a global context, and implications for clinical practice and future research studies are discussed. 


\section{ACKNOWLEDGEMENTS}

Top billing in this list of acknowledgements undoubtedly belongs to my husband, George. In the almost 14 years we have spent together, I have always considered myself unimaginably lucky to have found him and for him to have chosen me. This PhD odyssey has been incredibly difficult at times and without George, I do not know that I would have reached the end. If I had, I certainly would have been malnourished and absolutely miserable by now. His support has been unwavering and I am still amazed that he never once asked me, in the past four and a half years, when I was going to be finished. Despite the enormous impact this project has had on our lives and my constant anxiety about completing it, he has always simply said that it will be done when it is done. And now it is. Thank you my Gorgeous, I love you more each day.

I am indebted to my participants who trusted me with their stories, took the time to fill in my surveys, and who believed that this research would be of benefit to others. I hope I have done justice to their experiences and reflections. I am also grateful to all the individuals, groups, and organisations that disseminated information about my research to potential participants on my behalf.

My supervisor, Marc Wilson, has also been unfailingly supportive. He has patiently listened to me, constructively challenged me, advocated for me, and taught me. There were many times when, not having enough faith of my own, I had to rely on his faith in my abilities to sustain me. His consistent affirmation and CBT'esque objections to my self-doubt have been invaluable. Certainly, our afternoon sessions, spent discussing self-injury and many other vaguely relevant (but often tangential) topics, have been a highlight of this process for me.

Thank you also to my family; my parents, Joan and Graeme Anderson, who instilled in me a love of education and always supported my desire to continue learning, and my sisters, Lara and Kate, whose friendship I cherish. My parents-inlaw, Jane and Simon Langlands, also played an important support role in choosing 
to invest in a house with George and I, thus providing us with a very comfortable roof over our heads and a savings plan to boot.

I am also fortunate enough to have met and held onto some amazingly warm, smart, and funny friends. Thanks to Prabhat Sethi, Linda Rodenburg, Phil Cook, and Wendy Faust for being in my life. Special mention goes to Jessie Wilson and Erica Chadwick-kindred spirits whom I met while completing my $\mathrm{PhD}$. I know we will be lifelong friends.

At the risk of revealing myself as a "crazy cat lady", I have also appreciated my cats' love and company. Thanks to Wol, Fisher, and Tandem for all the times you distracted me from myself and this thesis by mewing for attention and affection; walking over my keyboard and typing random, strings of letters; and chasing the cursor.

Thanks to all the numerous other people who have helped me in various ways, shapes, and forms including: Monica Cartner, Bridget Greaney, Gaynor Parkin, Murray Patton, Egan Bidois, Gerard Hoffman, Moana Kerr, Garth Healey, Kuni Shepherd, Clive Banks, Lynne Russell, John McDowall, and Tony Ward. I also appreciated the insightful comments and suggestions provided by my three examiners - Dr Deirdre Brown (Victoria University of Wellington), Dr Cate Curtis (University of Waikato), and Dr David Klonsky (University of British Columbia).

Finally, I will always be grateful to the Tertiary Education Commission, the Freemasons Charity, and Victoria University of Wellington for the generous financial support I received to complete my PhD. 
List of tables $\quad$ xii

List of figures $\quad$ xiii

$\begin{array}{ll}\text { Thesis overview } & 1\end{array}$

Chapter one: What is non-suicidal self-injury? 4

1. INTRODUCTION 4

2. SELF-INJURY DEFINITIONS ARE SOCIO-CULTURALLY BOUND 5

2.1 Historical perspectives

$\begin{array}{ll}2.2 \text { Cross-cultural perspectives } & 7\end{array}$

3. WHAT COUNTS AS SELF-INJURY WITHIN WESTERN CULTURES? 8

3.1 Definitional debates $\quad 10$

3.2 Differentiating non-suicidal self-injury from suicide attempts $\quad 14$

3.2.1 Consumer Perspectives

$\begin{array}{ll}\text { 3.2.2 Professional Perspectives } & \mathbf{1 6}\end{array}$

3.3 Should self-injury be classified as a symptom or a syndrome? 18

$\begin{array}{lr}\text { 3.3.1 Self-injury as a symptom } & 19\end{array}$

$\begin{array}{ll}\text { 3.3.2 Self-injury as a syndrome } & 21\end{array}$

3.3.2.1 Proposed diagnostic criteria $\quad \mathbf{2 1}$

3.3.2.2 Is taxonomising self-injury within the DSM useful or necessary?

3.3.2.3 How would a NSSI disorder impact on non-Western cultures?

4. DEFINING SELF-INJURY FOR THE PURPOSE OF THIS THESIS 26

Chapter two: Why do people self-injure? 27

1. INTRODUCTION 27

2. HOW PREVALENT IS SELF-INJURY? 28

3. RISK FACTORS AND CORRELATES OF SELF-INJURY 29

$\begin{array}{ll}3.1 \text { Individual characteristics } & 29\end{array}$

3.1.1 Gender $\quad 29$

3.1.2 Ethnicity 31

3.1.3 Sexuality 33

3.2 Psychological characteristics $\quad 34$

3.2.1 Mental health disorders $\quad 34$

3.2.2 Suicidality

3.2.3 Temperament 38 
$\begin{array}{lr}\text { 3.2.4 Alexithymia } & 40\end{array}$

$\begin{array}{lr}\text { 3.3 Environmental characteristics } & 40\end{array}$

$\begin{array}{ll}\text { 3.3.1 Child maltreatment } & 40\end{array}$

3.3.2 Peer victimisation $\quad 42$

$\begin{array}{ll}3.4 \text { Summary and limitations } & 43\end{array}$

4. SELF-REPORTED REASONS AND MOTIVATIONS FOR SELF-INJURY 44

$\begin{array}{lr}4.1 \text { Intrapersonal Reasons } & 45\end{array}$

$\begin{array}{ll}\text { 4.1.1 Emotional reasons } & 45\end{array}$

4.1.1.1 Specific emotions $\quad 4 \mathbf{4 6}$

$\begin{array}{ll}\text { 4.1.1.2 Specific affect states } & \mathbf{4 7}\end{array}$

4.1.1.3 General emotional experiences $\quad 48$

$\begin{array}{lr}\text { 4.1.2 Cognitive reasons } & 48\end{array}$

$\begin{array}{ll}\text { 4.1.2.1 Specific cognitions } & 48\end{array}$

4.1.2.2 General cognitive states $\quad 49$

$\begin{array}{ll}\text { 4.1.3 Physiological reasons } & 50\end{array}$

4.1.3.1 Releasing pressure or tension $\quad \mathbf{5 0}$

4.1.3.2 Inducing stimulation $\quad 51$

$\begin{array}{ll}\text { 4.1.4 Self-punishment } & 52\end{array}$

4.2 Interpersonal Reasons $\quad 53$

$\begin{array}{ll}\text { 4.2.1 Support-seeking } & 53\end{array}$

$\begin{array}{ll}\text { 4.2.2 Avoidance } & 54\end{array}$

$\begin{array}{lr}4.3 \text { Limitations } & 54\end{array}$

$\begin{array}{lr}\text { 4.4 Summary } & 56\end{array}$

5. SELF-REPORTED ANTECEDENTS AND CONSEQUENCES OF SELF- 57 INJURY

$\begin{array}{lr}5.1 \text { Retrospective studies } & 58\end{array}$

$\begin{array}{lr}5.2 \text { Prospective studies } & 59\end{array}$

$\begin{array}{lr}5.3 \text { Summary and limitations } & 60\end{array}$

6. LABORATORY STUDIES $\quad 61$

$\begin{array}{ll}\text { 7. CONCLUSION } & 63\end{array}$

Chapter three: The functions of self-injury: Theoretical 66 conceptualisations

$\begin{array}{ll}\text { 1. INTRODUCTION } & 66\end{array}$

2. SINGLE-FUNCTION MODELS OF SELF-INJURY 68

2.1 Affect regulation $\quad 68$

2.2 Self-punishment $\quad 70$

2.3 Anti-dissociation $\quad 71$

2.4 Anti-suicide $\quad 72$

2.5 Sexual $\quad 73$

$\begin{array}{ll}2.6 \text { Sensation-seeking } & 74\end{array}$

2.7 Interpersonal boundaries $\quad 74$ 
3. MULTI-FUNCTION MODELS OF SELF-INJURY 76

3.1 The Four Functions Model $\quad 76$

$\begin{array}{ll}\text { 3.1.1 Assumptions of the FFM } & 76\end{array}$

$\begin{array}{ll}\text { 3.1.2 Empirical support for the FFM } & 78\end{array}$

3.2 The Experiential Avoidance Model $\quad 80$

$\begin{array}{lr}\text { 3.2.1 Assumptions of the EAM } & 80\end{array}$

3.2.2 Empirical support for the EAM

3.3 Evaluating the FFM and the EAM $\quad 86$

$\begin{array}{ll}\text { 3.3.1 Predictive accuracy } & 86\end{array}$

$\begin{array}{ll}\text { 3.3.2 Internal coherence } & 87\end{array}$

$\begin{array}{lr}\text { 3.3.3 External consistency } & 89\end{array}$

$\begin{array}{lr}\text { 3.3.4 Unifying power } & 89\end{array}$

$\begin{array}{lr}\text { 3.3.5 Fertility } & 90 \\ 3.3 .6 \text { Simplicity } & 90\end{array}$

$\begin{array}{lr}3.3 .6 \text { Simplicity } & 90\end{array}$

4. THE CURRENT THESIS $\quad 91$

Chapter four: An Interpretative Functional Analysis of selfinjury

1. INTRODUCTION 94

2. STUDY OVERVIEW 96

3. STAGE ONE: SCREENING FOR SELF-INJURIOUS BEHAVIOURS 97

$\begin{array}{ll}3.1 \text { Method } & 97\end{array}$

$\begin{array}{ll}\text { 3.1.1 Recruitment strategy } & 97\end{array}$

$\begin{array}{lr}\text { 3.1.2 Participants and procedure } & 99\end{array}$

$\begin{array}{lr}\text { 3.1.3 The Deliberate Self-harm Inventory } & 100\end{array}$

$\begin{array}{ll}3.2 \text { Results } & 101\end{array}$

$\begin{array}{ll}\text { 3.2.1 Demographic and diagnostic information } & 101\end{array}$

$\begin{array}{ll}\text { 3.2.2 Prevalence of different types of self-injury } & 101\end{array}$

4. STAGE TWO: THE INTERVIEWS 105

$\begin{array}{ll}\text { 4.1 Participants and procedure } & 105\end{array}$

$\begin{array}{ll}\text { 4.2 Analysing the interview transcripts } & 109\end{array}$

$\begin{array}{ll}\text { 4.2.1 What is a functional analysis? } & \mathbf{1 1 0}\end{array}$

4.2.2 Utilising functional analyses with typically developing populations 110

$\begin{array}{ll}\text { 4.2.3 Interpretative Functional Analysis } & 112\end{array}$

4.2.3.1 Rationale $\quad \mathbf{1 1 2}$

4.2.3.2 Epistemological assumptions $\quad \mathbf{1 1 4}$

$\begin{array}{lr}\text { 4.2.3.3 Coding system } & \mathbf{1 1 4}\end{array}$

4.2.3.3.1 Relevant learning history $\quad \mathbf{1 1 5}$

4.2.3.3.2 Antecedents $\quad \mathbf{1 1 5}$

$\begin{array}{ll}\text { 4.2.3.3.3 Target Behaviour } & \mathbf{1 1 7}\end{array}$ 
4.2.3.3.4 Consequences $\quad \mathbf{1 1 7}$

4.2.3.3.5 Escape or access: Functions of the target behaviour $\quad \mathbf{1 1 8}$

4.2.3.4 Steps in the Interpretative Functional Analysis $\quad \mathbf{1 1 8}$

4.3 Interview results

121

4.3.1 Intrapersonal escape/avoidance 122

4.3.1.1 Establishing operations $\quad \mathbf{1 2 3}$

4.3.1.2 Discriminative stimuli $\quad 131$

4.3.1.3 Consequences $\quad \mathbf{1 3 5}$

4.3.1.4 Summary $\quad 139$

4.3.2 Interpersonal escape/avoidance 139

$\begin{array}{ll}\text { 4.3.3 Intrapersonal access } & \mathbf{1 4 0}\end{array}$

4.3.3.1 Establishing operations and discriminative stimuli $\quad \mathbf{1 4 1}$

4.3.3.2 Consequences

4.3.3.3 Summary $\quad \mathbf{1 4 4}$

$\begin{array}{ll}\text { 4.3.4 Interpersonal access } & \mathbf{1 4 5}\end{array}$

$\begin{array}{lr}\text { 4.3.4.1 Establishing operations } & \mathbf{1 4 5}\end{array}$

$\begin{array}{lr}\text { 4.3.4.2 Discriminative stimuli } & 147\end{array}$

$\begin{array}{lr}\text { 4.3.4.3 Consequences } & \mathbf{1 4 8}\end{array}$

4.3.4.4 Summary $\quad \mathbf{1 5 0}$

$\begin{array}{ll}\text { 4.3.5 Aversive consequences } & 150\end{array}$

4.3.5.1 Negative emotions and thoughts $\quad \mathbf{1 5 0}$

4.3.5.2 The wound and scarring $\quad \mathbf{1 5 2}$

4.3.5.3 Restricted clothing choices $\quad \mathbf{1 5 3}$

4.3.5.4 Reactions from others $\quad \mathbf{1 5 4}$

4.3.5.5 Exclusive experience of punishing consequences $\mathbf{1 5 6}$

4.3.5.6 Summary $\mathbf{1 5 6}$

4.3.6 Summary of Interpretative Functional Analysis results

5. STAGE THREE: EMAIL OR TELEPHONE FOLLOW-UP 157

6. STAGE FOUR: FEEDBACK QUESTIONNAIRE 158

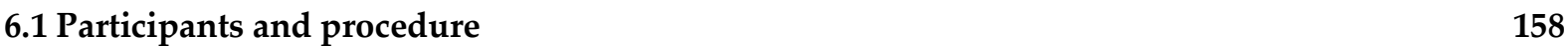

$\begin{array}{ll}\text { 6.2 Measure } & 158\end{array}$

6.3 Results $\quad 159$

$\begin{array}{ll}\text { 7. DISCUSSION } & 161\end{array}$

7.1 Forms and frequencies of self-injurious behaviours 161

7.2 Is self-injury primarily an experientially avoidant behaviour within Aotearoa New 162

Zealand?

7.2.1 The role of cognitions 163

$\begin{array}{ll}\text { 7.2.2 What about positive reinforcement? } & 165\end{array}$

7.3 Strengths of Interpretative Functional Analysis $\quad 166$

7.4 Limitations of Interpretative Functional Analysis $\quad 166$

$\begin{array}{ll}7.5 \text { Is participating in a study about self-injury harmful? } & 167\end{array}$

$\begin{array}{ll}7.6 \text { Conclusion } & 169\end{array}$

Chapter five: Quantifying the functions of self-injury 170

1. INTRODUCTION 
2. METHOD 171

$\begin{array}{lr}2.1 \text { Procedure } & 171\end{array}$

$\begin{array}{ll}2.1 .1 \text { Recruitment strategy } & 171\end{array}$

$\begin{array}{ll}\text { 2.1.2 Inclusion/exclusion criteria } & 173\end{array}$

$\begin{array}{ll}\text { 2.1.3 General ethical considerations } & 173\end{array}$

2.1.4 Ethical issues specific to Internet Mediated Research 173

2.1.4.1 Meeting the inclusion criteria $\quad \mathbf{1 7 4}$

2.1.4.2 Exiting the survey before being debriefed $\quad \mathbf{1 7 4}$

2.1.4.3 Protecting anonymity 175

2.2 Participants

175

2.2.1 Flow of participants through the study 175

2.2.2 Demographics and descriptive characteristics of the sample 177

2.3 Measures

179

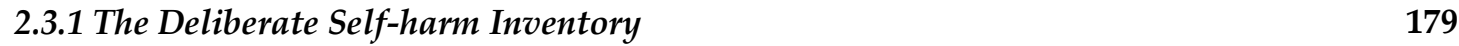

2.3.2 Inventory of Statements about Self-injury 179

2.3.3 Positive and Negative Affect Schedule 181

2.3.4 Revised Automatic Thoughts Questionnaire 182

$\begin{array}{lr}2.4 \text { Open-ended questions } & 183\end{array}$

3. QUANTITATIVE RESULTS 183

3.1 Characteristics of participants' self-injurious behaviour 183

$\begin{array}{lr}\text { 3.1.1 Global NSSI episodes } & 184\end{array}$

3.1.2 Most recent NSSI episodes 186

$\begin{array}{lr}3.2 & \text { Is affect regulation the primary function of NSSI? } \\ \end{array}$

3.2.1 Self-reported functions of participants' global NSSI episodes 188

3.2.2 Self-reported functions of participants' most recent NSSI episode 190

3.3 Are intrapersonal functions more highly endorsed then interpersonal? 192

$\begin{array}{ll}\text { 3.3.1 Cluster analyses } & 193\end{array}$

3.3.1.1 Cluster analysis of global NSSI functions 193

3.3.1.2 Cluster analysis of participants' most recent NSSI episode 195

3.3.2 Comparing intrapersonal and interpersonal functions 196

3.4 Do people report a decrease in negative affect and an increase in positive affect 198

following NSSI?

3.5 Does the content of people's cognitions change following NSSI? 201

$\begin{array}{lr}3.6 \text { Summary of quantitative findings } & 202\end{array}$

4. OPEN-ENDED RESPONSES 2203

$\begin{array}{lr}\text { 4.1 Antecedents of NSSI } & 203\end{array}$

4.2 Thematic Analysis of the consequences of NSSI $\quad 205$

$\begin{array}{ll}\text { 4.2.1 Self becomes transgressor } & 206\end{array}$

4.2.1.1 Concealing transgressions from others $\quad 208$

4.2.1.2 Being judged by others for transgressions $\quad 210$

4.2.1.3 Transgressions cause other to suffer $\quad \mathbf{2 1 1}$

$\begin{array}{ll}\text { 4.2.2 Self becomes helper } & 211\end{array}$

4.2.2.1 Regulating emotions $\quad 212$

4.2.2.2 Accessing support and/or treatment $\quad 214$

4.2.2.3 The physical wound $\quad 215$

$\begin{array}{ll}\text { 4.3 Summary of findings from the open-ended responses } & 216\end{array}$

$\begin{array}{ll}\text { 5. DISCUSSION } & 216\end{array}$ 
5.1 Affect regulation and self-punishment are the primary single functions of NSSI 216

$\begin{array}{ll}5.2 \text { Intrapersonal functions are more highly endorsed than interpersonal functions } & 218\end{array}$

5.3 Negative affect decreased and positive affect increased following NSSI 219

$\begin{array}{lr}5.4 \text { Negative cognitions decreased following NSSI } & 220\end{array}$

$\begin{array}{lr}5.5 \text { Strengths } & 221\end{array}$

$\begin{array}{lr}5.6 \text { Limitations } & 222\end{array}$

$\begin{array}{lr}5.7 \text { Conclusion } & 222\end{array}$

Chapter six: Comparing the intrapersonal experiences and coping styles of people with and without a history of NSSI

$\begin{array}{lr}2.3 \text { Measures } & 229\end{array}$

2.3.1 The Deliberate Self-harm Inventory 1229

2.3.2 The Acceptance and Action Questionnaire $\quad 229$

2.3.3 The Positive and Negative Affect Schedule 230

2.3.4 The White Bear Suppression Inventory 230

2.3.5 The Depression Anxiety Stress Scale $\quad 231$

2.3.6 The Automatic Thoughts Questionnaire - short version 231

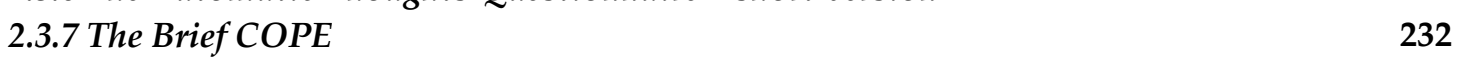

$\begin{array}{lr}\text { 3. RESULTS } & 233\end{array}$

3.1 Prevalence and frequencies of self-injury types $\quad 233$

3.2 Do people who have self-injured experience more negative emotions and thoughts? 235

3.3 Do people who have self-injured have a greater tendency towards avoidant coping? 241

3.4 Is NSSI predicted by negative emotions and thoughts? $\quad 244$

3.5 Does avoidance mediate relationships between negative intrapersonal experiences 248 and NSSI?

\section{DISCUSSION}

4.1 Experience of negative emotions and thoughts $\quad 251$

4.2 Tendencies towards avoidant coping $\quad 252$

4.3 Do negative emotions and thoughts cause self-injury? 253

4.4 Specific types of avoidance may underlie self-injury $\quad 254$

$\begin{array}{lr}4.5 \text { Strengths } & 255\end{array}$

$\begin{array}{lr}\text { 4.6 Limitations } & 255\end{array}$

$\begin{array}{lr}4.7 \text { Conclusions } & 255\end{array}$

Chapter seven: General discussion 
6. WHERE TO FROM HERE?

6.1 More sophisticated methods are necessary experiential avoidance?

6.5 How do functions of self-injury change over time?

References

\section{Appendices (on CD attached to back cover)}

Appendix A: Information sheet

Appendix B: Screening survey

Appendix C: Support organisations

Appendix D: Interview questions

Appendix E: Consent to contact clinician

Appendix F: Consent form

Appendix G: Evaluation of research participation

Appendix H: Online survey, including information and debriefing sheets

Appendix I: Coding scheme

Appendix J: Debriefing information (for email)

Appendix K: Online survey (Times 1 and 2)

Appendix L: Options for support 
Table 1. Contrasting definitions and referents of the three most commonly used terms for self-injurious behaviours

Table 2. Frequencies and recency of different types of NSSI

Table 3. Number of participants $(N=23)$ who endorsed each statement

Table 4. Frequencies of different types of NSSI

Table 5. Frequencies of different types of NSSI for participants' most recent episode

Table 6. Descriptive statistics and reliability coefficients for the ISAS in reference to participants' global and most recent episodes of NSSI

Table 7. Agglomeration schedule, Ward's method and squared Euclidean distance for global NSSI functions

Table 8. Agglomeration schedule, Ward's method and squared Euclidean distance for functions of participants' most recent NSSI episode

Table 9. Descriptive statistics and reliability coefficients for the PANAS-X subscale responses before and after participants' most recent episode of NSSI

Table 10. Ranks for PANAS-X subscales before and after participants' most recent episode of NSSI

Table 11. Antecedent event categories with percentage endorsement and qualitative examples

Table 12. Frequencies of NSSI types reported at Times 1 and 2

Table 13. NSSI recency at Times 1 and 2

Table 14. Time 1 reliability coefficients, descriptive statistics, and ANOVAs for the No NSSI versus Lifetime NSSI groups

Table 15. Two binary logistic regression models for NSSI versus No NSSI 


\section{LIST OF FIGURES}

$\begin{array}{ll}\text { Figure 1. } & \text { The elaborated FFM. }\end{array}$

$\begin{array}{lll}\text { Figure 2. } & \text { The Experiential Avoidance Model. } & 81\end{array}$

Figure 3. Functional schematic of Melanie's most recent episode of NSSI. 120

Figure 4. Functions fulfilled by each participant's most recent episode of NSSI. $\quad 121$

Figure 5. Flow of participants through the online survey. 176

Figure 6. Dendrogram using Ward linkage for functions of participants' global 194 episodes of NSSI.

Figure 7. Dendrogram using Ward linkage for functions of participants' most recent NSSI episode.

Figure 8. Thematic Map.

Figure 9. Flow of participants through the study at Times 1 and 2.

Figure 10. Cross-lagged panel model of NSSI and global negative affect.

Figure 11. Cross-lagged panel model of NSSI and negative automatic thoughts.

Figure 12. Cross-lagged panel model of NSSI and hostility.

Figure 13. Cross-lagged panel model of NSSI and guilt.

Figure 14. Cross-lagged panel model of NSSI and fear.

Figure 15. Cross-lagged panel model of NSSI and sadness.

Figure 16. Unstandardised regression coefficients for the relationship between negative affect and NSSI which is not mediated by experiential avoidance.

Figure 17. Unstandardised regression coefficients for the relationship between negative affect and NSSI which is partially mediated by thought suppression.

Figure 18. Unstandardised regression coefficients for the relationship between fear and NSSI which is not mediated by experiential avoidance. 
Figure 19. Unstandardised regression coefficients for the relationship between fear and NSSI which is partially mediated by thought suppression.

Figure 20. Unstandardised regression coefficients for the relationship between hostility and NSSI which is not mediated by experiential avoidance.

Figure 21. Unstandardised regression coefficients for the relationship between hostility and NSSI which is partially mediated by thought suppression.

Figure 22. Unstandardised regression coefficients for the relationship between guilt and NSSI which is not mediated by experiential avoidance.

Figure 23. Unstandardised regression coefficients for the relationship between guilt and NSSI which is not mediated by thought suppression.

Figure 24. Unstandardised regression coefficients for the relationship between sadness and NSSI which is not mediated by experiential avoidance.

Figure 25. Unstandardised regression coefficients for the relationship between sadness and NSSI which is partially mediated by thought suppression.

Figure 26. Unstandardised regression coefficients for the relationship between automatic thoughts and NSSI which is not mediated by experiential avoidance.

Figure 27. Unstandardised regression coefficients for the relationship between automatic thoughts and NSSI which is partially mediated by thought suppression.

Figure 28. NSSI within an adaptive coping hierarchy. 


\section{THESIS OVERVIEW}

Answering the question of whether Non-Suicidal Self-Injury (NSSI) functions primarily as a experientially avoidant behaviour within Aotearoa New Zealand first necessitated an extensive review of the extant literature about how NSSI is defined, how it functions, and how it is conceptualised within behavioural models of selfinjury. This review comprises the first three chapters of my thesis. In Chapter 1, I consider how definitions of self-injurious behaviours are socio-culturally bound, focusing in particular on the ways in which what counts as self-injury within Western cultures has been shaped by both consumer and professional perspectives. I present the evidence in support of NSSI being distinguished from suicide attempts, and critique suggestions that NSSI should be classified as a syndrome, rather than symptom, of psychopathology. To conclude the chapter, I define the parameters of NSSI for the purpose of my thesis.

My second chapter concentrates on why people self-injure. I review the current evidence base about risk factors and correlates of NSSI, including individual, psychological, and environmental characteristics. Following this detailed examination of the factors that are associated with, or predict, self-injury, I delineate the most commonly reported intrapersonal (i.e., emotional, cognitive, and physiological) and interpersonal (i.e., support-seeking and avoidance) reasons for self-injury. Literature detailing the self-reported antecedents and consequences of NSSI is also reviewed, along with laboratory studies about why people self-injure.

Building on the evidence that I present in Chapter 2, I then move on to discuss functional models of NSSI in Chapter 3. Individual reasons, antecedents, and consequences of self-injury are typically grouped into an array of single function models. I review the literature in support of the eight most common single-function models and discuss the limitations of such conceptualisations of NSSI, before outlining and critiquing two multi-function models - the Four Functions Model (Nock, 2008; Nock \& Prinstein, 2004, 2005) and the Experiential Avoidance Model 
(EAM; Chapman, Gratz, \& Brown, 2006) - according to well-established epistemic values. I close the chapter with a summary of the empirical studies that I conducted for the purpose of this thesis.

Chapter 4 details the first empirical study I conducted to test whether NSSI functions primarily as an experientially avoidant behaviour within Aotearoa New Zealand. This interview study consisted of four stages: (1) screening potential participants for self-injurious behaviours, (2) interviewing participants about the antecedents and consequences of their most recent episode of NSSI, (3) following up with participants subsequent to the interviews, and (4) distributing a research evaluation questionnaire to interview participants. The methods and results of these four stages are presented and discussed in Chapter 4.

My fifth chapter describes the findings of a nation-wide survey I conducted to quantitatively test the assumptions of the EAM in a larger sample of people living in Aotearoa New Zealand. More specifically, I examined whether affect regulation is the primary function of NSSI, whether intrapersonal functions are more highly endorsed than interpersonal functions, and whether the valence of people's emotions and the content of their cognitions changes following NSSI. A series of open-ended questions, contained within the survey, about the antecedents and consequences of participants' most recent episode of NSSI are also qualitatively analysed and discussed.

In Chapter 6, the final empirical chapter of my thesis, I compare the intrapersonal experiences and coping styles of people who have self-injured with those who have never engaged in self-injurious behaviour across two time-points. I present and discuss a series of analyses conducted to test the following hypotheses: (1) people with a history of NSSI will experience more negative emotions and thoughts, and evidence a greater tendency towards avoidant coping than people without a history of NSSI, (2) NSSI will be predicted by negative emotions and thoughts, and (3) avoidance will mediate the relationships between negative intrapersonal experiences and NSSI. 
I conclude my thesis in Chapter 7 by discussing the most pertinent findings from my three studies and considering how my research relates to the broader conceptual issues outlined in Chapter 1, such as whether NSSI should be classified as a mental disorder. Finally, before outlining how future research can build on the strengths and limitations of my own studies, I reflect on the clinical implications of my research. 


\section{CHAPTER ONE}

\section{What is non-suicidal self-injury?}

"We express our distress through bodily idioms that are both peculiar to distinctive cultural worlds and constrained by our shared human condition." (Kleinman, 1988, p. xiii)

\section{INTRODUCTION}

"I am a cutter." This deceptively simple phrase is how one of my participants described himself at the start of our interview. Sitting across from me with rolled-up sleeves to expose his heavily scarred forearms, I wondered how and why cutting had become a defining aspect of his identity. What did those scars mean to him and what did they communicate to others in his community, given that self-injury exists firmly outside of the boundaries of what is deemed to be socially acceptable behaviour within Aotearoa New Zealand?

Understanding what factors lead to Non-Suicidal Self-Injury (NSSI), why selfinjurious behaviours become entrenched over time, and how people make sense of self-injury is complicated because NSSI is a multi-faceted behaviour, that "cannot be understood without reference to biological, psychological, social, and cultural components" (Favazza \& Rosenthal, 1993, p. 138). As a Clinical Psychologist in training, my focus in this thesis is on clarifying the psychological factors that trigger, reinforce, and maintain self-injurious behaviours in populations from Aotearoa New Zealand. Although I touch on biological factors in my discussion of the psychology of self-injury, they are, by and large, beyond the scope of this thesis.

Social and cultural norms, however, while not explicitly investigated in my studies do warrant an in-depth discussion at this stage of my thesis because these norms determine what counts as NSSI and, as a result, which behaviours I examine in this research. Every person who self-injures is situated in a particular sociocultural context, which influences the meaning of self-injury to that person and others in their community. It is therefore imperative, before I begin to delve into the theoretical and empirical research that constitutes my thesis, to consciously reflect on 
how the socio-cultural norms within Aotearoa New Zealand and other Western countries have shaped the way in which the self-injurious behaviours under investigation are defined.

Before focusing on how self-injurious behaviours are currently conceptualised in Western societies, I briefly discuss the importance of recognising that definitions of self-injury are social constructs, which shift across time and between cultures. What counts as self-injury within contemporary, Western socio-cultural contexts has been shaped by both professionals and mental health consumers. Two definitional questions, in particular, have been extensively debated in the literature on selfinjurious behaviours: (1) Can self-injury be distinguished from suicidal behaviours? (2) Should self-injury be categorised as a symptom of mental illness or a separate clinical syndrome? (Cresswell, 2005). After discussing the evidence that self-injury can be differentiated from suicide attempts and the rationale behind classifying NSSI as a syndrome rather than symptom, I conclude this chapter by outlining the definition of self-injurious behaviour used in this thesis.

\section{SELF-INJURY DEFINITIONS ARE SOCIO-CULTURALLY BOUND}

Two ways of exploring how current socio-cultural contexts shape our understanding of NSSI are to reflect on how this understanding has shifted over time and to compare our Western conceptualisations of self-injury with those of other cultures. I concur with Watters (2010) who argues that, "Symptoms of mental illnesses are the lightning in the zeitgeist, the product of culture and belief in specific times and specific places" (p. 3). Exploring historical and cross-cultural perspectives on NSSI, even though it is not always conceptualised as a symptom of psychopathology, can help us to illuminate the underlying assumptions and values that currently dominate our thinking about self-injurious behaviours (Watters, 2010).

\subsection{Historical perspectives}

Browsing through journal articles on self-injury, it quickly becomes apparent that our knowledge about NSSI in Western cultures has evolved significantly within 
past decades. For example, it is hard to fathom a journal editor today publishing the following strikingly pejorative and reductive description of women who self-injure:

In summary, the cutter is an attractive, intelligent, unmarried young woman, who is either promiscuous or overly afraid of sex, easily addicted, and unable to relate successfully to others. She is an older one in a group of siblings with a cold, domineering mother and a withdrawn, passive, hypercritical father. She slashes her wrists indiscriminately and repeatedly at the slightest provocation, but she does not commit suicide. She feels relief with the commission of her act. (Graff \& Mallin, 1967, p. 38)

This extract is objectionable for many reasons: the use of blaming, invalidating statements ("slightest provocation"), the blatant contradictions ("promiscuous" versus frigid), the characterisation of parents as villains, the use of stigmatising language such as "cutter" and "slashes", and the unnecessary attention to physical features ("attractive"), intelligence, and marital status. Furthermore, the overly confident, sweeping assertions made in this description are astounding considering that this research was based on only 20 female patients; the authors excluded the one male because they "felt he was atypical" (Graff \& Mallin, 1967, p. 36).

Of course, the socio-cultural context in which such historical accounts were produced was vastly different to the environment we currently inhabit. For a start, Psychoanalysis was the dominant therapeutic modality; understandings of what selfinjury was and who self-injured were largely governed by this theoretical framework (Shaw, 2002). In contrast, the contemporary emphasis on evidence-based treatments (i.e., Dialectical Behavioural Therapy [DBT]; Linehan, 1993a) has shaped current conceptualisations of NSSI to focus on behavioural functions (Shaw, 2002).

Second, research on NSSI began proliferating from the mid-1980s, a trend that has since continued unabated (Nock, 2010; Shaw, 2002). With the increase in publications on self-injury, the myth of the typical self-injurer (Hodgson, 2004) has been dispelled. Current perspectives on NSSI acknowledge the complexity of selfinjurious behaviours, which are carried out by both males and females (Andover, Primack, Gibb, \& Pepper, 2010; Claes, Houben, Vandereycken, Bijttebier, \& Muehlenkamp, 2010), manifest across diverse cultures (Brausch \& Gutierrez, 2010), 
and associated with an array of risk factors and psychopathological correlates (Fliege, Lee, Grimm, \& Klapp, 2009; Gratz, 2003; Nock, 2009).

Third, the mid-1980s witnessed the advent of the psychiatric survivor movement whose members actively challenged clinical constructions of selfinjurious behaviours. The role of mental health consumers in defining what NSSI is (and what it is not) will be discussed in more detail later in this chapter, but it is worth noting here that consumer testimonies about self-injury have exerted considerable influence in the past three decades on both practitioners' and the general public's perspectives of NSSI (Cresswell, 2005).

Finally, recent technological advances have not only altered the way in which both lay and academic information about self-injury is disseminated, but also the quality and quantity of that information. In particular, the Internet facilitates access to support groups for people who self-injure, along with stories, graphic video clips, and clinical information about NSSI, all of which can contribute to the normalising or "narrative reinforcement" of these behaviours within certain communities (Whitlock, Lader, \& Conterio, 2007, p. 1139). Undoubtedly, this interactive, online environment, which simply did not exist in the past, has influenced the way in which self-injury is understood on an individual and collective level.

\subsection{Cross-cultural perspectives}

Until recently, psychological or psychiatric research with people from nonWestern cultures who self-injure was virtually non-existent. Twentieth century academic investigations of NSSI almost exclusively document self-injurious behaviours among middle-class, white women (Shaw, 2002); Favazza's seminal work on the interplay between culture and self-injury, which was first published in 1987, is one of the major exceptions. Examining the relationships between what Favazza (2011) terms culturally sanctioned body modification and pathological self-injury, brings into clear focus the role of culture in determining not only what types of selfinjury are carried out, but also what meaning is ascribed to these behaviours and how that meaning is communicated to others. 
Both socially acceptable and unacceptable self-injurious behaviours are posited to "serve an identical purpose, namely, an attempt to correct or prevent a pathological, destabilizing condition that threatens the community, the individual, or both" (Favazza, 1996, p. 222). Self-injury is a preventative or remedial activity that allows individuals and communities to create order out of perceived or impending chaos and, as such, examples of ritualistic, religious, and mythic self-injury abound in both Western and Non-Western cultural practices and narratives (see Favazza, 2011 for a detailed review).

Given the importance of culture in demarcating the limits of socially acceptable self-injury, the rigorous application of current Western classification systems to Non-Western cultures would inevitably pathologise culturally appropriate self-injurious behaviours. For example, Australian Aboriginal peoples traditionally cut themselves when in mourning (Farrelly \& Francis, 2009; Sheldon, 2001). Such "sorry cuts" are not only socially accepted but may be expected in Aboriginal communities as an expression of grief (Sheldon, 2001, p. 440). These behaviours form a marked contrast to socially acceptable grieving practices within Western cultures.

Certainly, it is instructive to contemplate how the treatment of Westerners who cut themselves would change if these behaviours were reframed to become socially acceptable "distress cuts". While I am not advocating that this should be the case, it is possible that any self-stigma (Ben-Zeev, Young, \& Corrigan, 2010) engendered by a history of self-injurious behaviours, which could in turn fuel subsequent episodes, may be ameliorated if these behaviours were consistently viewed as adaptive responses to distress. Indeed, such a reframing is what consumer activists have attempted to achieve (Pembroke, 1996a).

\section{WHAT COUNTS AS SELF-INJURY WITHIN WESTERN CULTURES?}

It is clear that socio-cultural norms play a fundamental role in how NSSI is defined; however, a shared socio-cultural context does not necessarily imply consensus. What counts as NSSI ultimately depends on who is doing the defining: 
Acts of self-injury can hold specific meanings for particular individuals or subgroups who may choose to accept, revise, or challenge hegemonic social and cultural understandings.

In seeking to unpack the complexities and contradictions inherent in how selfinjurious behaviours are defined and classified within Western cultures, I stumbled upon the burgeoning study of personal epistemology. Broadly speaking, personal epistemology is concerned with how people define, construct, and evaluate knowledge in order to make sense of the world around them (Hofer, 2002). Although the field of personal epistemology is not without its own controversies around what are highly abstract and complex concepts (Schommer-Aikins, 2002), my critique, by necessity, is simply informed by this field and does not seek to delve into or resolve these debates.

Personal epistemologies are extremely relevant to the question of what counts as NSSI within Western cultures; how researchers, clinicians, and mental health consumers define and categorise self-injury depends on their ways of knowing and what forms of knowledge they privilege. The contrast between empirical knowledge, favoured by professionals, and experiential knowledge, favoured by consumers, is highlighted by Cresswell (2005): “'Official' knowledge stresses scientific classification, professional expertise, and statistical evidence: 'Survivor' knowledge, by contrast, emphasises individual experience, the traumas of the life-course, and the personal testimony of the survivor as itself expert data" ${ }^{1}$ (p. 1668). By analysing the accounts of self-harm survivors published in Self-harm: Perspectives from personal experience, Cresswell demonstrates how some people actively resist and reject psychiatric hegemony through producing visceral testimonies about survival through self-injury. These testimonies function as a form of political practice designed to challenge and remediate professional conceptualisations of self-injury (Cresswell, 2005).

\footnotetext{
${ }^{1}$ Cresswell (2005) uses the word "official" as a synonym for psychiatric.
} 
Given that many of these testimonies are directed at bio-medical approaches to NSSI, survivor activists are not necessarily challenging all professional depictions of self-injury. Furthermore, many of those who self-injure do not choose to become activists or even to align themselves with this movement: "People vary in the resources available to them to resist or rework the cultural meanings of illness" (Kleinman, 1988, p. 26). However, those that do choose to challenge 'official' knowledge production are able to locate themselves within the system as the creators, rather than simply the recipients, of knowledge (Clinchy, 2002) and thus begin to wield the power to influence collective understandings of NSSI. For the remainder of this chapter, I will examine how the interplay between professional and consumer epistemologies have shaped the current understanding of NSSI within Western cultures, and how discussions about ways to define and classify selfinjury have informed the operationalisation of NSSI for the purpose of this thesis.

\subsection{Definitional debates}

Cutting, self-mutilation, self-wounding, self-abuse, self-injury, deliberate selfharm (DSH), and self-inflicted violence are just a selection of the terms that have been used to denote self-injurious behaviours within academic literature. ${ }^{2}$ Most writers acknowledge the lack of consensus on how self-inflicted, harmful behaviours should be defined and commonly present their own definitions. As is evident in Table 1, this limits comparability of NSSI research findings because these definitions typically draw on idiosyncratic combinations of referents, including: self-inflicted, deliberate/purposeful, direct, suicidal intent, lethality, severity, tissue damage, function, and social acceptability.

This inconsistent use of referents has resulted in the same term (e.g., NSSI) being defined in multiple ways, while different terms (e.g., NSSI and DSH) are given

\footnotetext{
${ }^{2}$ To ensure consistency throughout this thesis, I generally refer to all non-suicidal self-injurious behaviours as NSSI even if this is not the term originally used by the author(s). For example, if authors use the term deliberate self-harm but their definition is consistent with that of non-suicidal self-injury, I refer to the behaviours as NSSI (with the exception of direct quotations). When authors have not distinguished between behaviours on the basis of suicidal intent, I refer to this as self-harm.
} 
Table 1

Contrasting definitions and referents of the three most commonly used terms for self-injurious behaviours

\section{Referents}

\section{Definition}

\begin{tabular}{|c|c|c|c|c|c|c|c|c|}
\hline \multicolumn{9}{|c|}{ Referents } \\
\hline 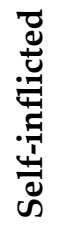 & 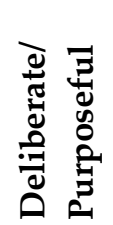 & 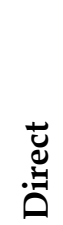 & 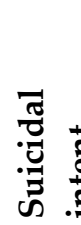 & 节 & 苞 & 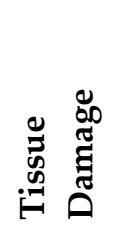 & 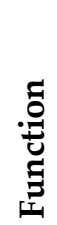 & (ت) \\
\hline
\end{tabular}

\section{Self-injury}

"Self-injury is intentional, self-effected, low-lethality bodily harm of a socially unacceptable nature,

performed to reduce psychological distress" (Walsh, 2006, p. 4).

"Non-suicidal self-injury (NSSI) is defined as the deliberate destruction of one's body tissue without

suicidal intent and for purposes not socially sanctioned" (Klonsky \& Glenn, 2009, p. 215).

"Nonsuicidal self-injury refers to purposeful, non-life-threatening self-inflicted injuries without suicidal

intent that aim to alleviate emotional distress" (Kokaliari \& Berzoff, 2008, p. 259).

\section{Self-harm}

"Deliberate self-harm may be defined as the deliberate, direct destruction or alteration of body tissue,

without conscious suicidal intent but resulting in injury severe enough for tissue damage to occur"

(Gratz, 2003, p. 192).

“Deliberate self-harm includes any intentional act of self-injury or self-poisoning (overdose), irrespective of the apparent motivation or intention" (Hawton \& Rodham, 2006, p. 11).

"Self-harm can be defined as socially unacceptable, intentional alteration or destruction of body tissue without conscious suicidal intent" (Croyle \& Waltz, 2007, p. 332)

\section{Self-mutilation}

"Self-mutilation refers to a complex group of behaviours in which there is deliberate destruction or alteration of body tissue without conscious suicidal intent" (Favazza, 1989, p. 113).

"Self-mutilative behavior (SMB) refers to the direct and deliberate destruction of one's own body tissue without suicidal intent" (Nock \& Prinstein, 2005, p. 140).

"A theoretical definition of self-mutilation is the intentional act of tissue destruction with the purpose of shifting overwhelming emotional pain to a more acceptable physical pain" (Hicks \& Hinck, 2008, p. 412). 
the same or similar definitions. For example, Gratz's (2003) definition of DSH "as the deliberate, direct destruction or alteration of body tissue, without conscious suicidal intent but resulting in injury severe enough for tissue damage to occur" (p. 192) essentially describes the same behaviours as Klonsky and Glenn's (2009) definition of NSSI as "the deliberate destruction of one's body tissue without suicidal intent and for purposes not socially sanctioned" (p. 215).

It is hardly surprising that, given the disparate use of terminology and accompanying referents, varying levels of agreement exist between professionals and mental health consumers as to the empirical and experiential validity of selfinjury definitions. Use of certain referents in defining self-injury is undisputed; for example, few researchers, clinicians, or consumers would argue against self-injury being purposeful and self-inflicted. Including the word deliberate, however, has been criticised because it implies "premeditation and wilfulness" (Pembroke, 1996b, p. 2) and insinuates that the person who self-injures is to blame for their behaviour (Taylor, 2003).

Another referent that is seldom disputed in definitions or studies of nonsuicidal self-injurious behaviours is direct. A distinction is typically drawn between direct, self-inflicted, purposeful behaviours where the damage is immediate (e.g., cutting) and indirect behaviours where the damage accrues over time (e.g., eating disorders) (Walsh, 2006). Aside from the temporal qualities of the damage, Walsh (2006) asserts that another difference between indirect and direct self-injury is that people who engage in indirect self-injury seldom do so with the explicit aim of hurting themselves. For example, someone with an eating disorder is more likely to report being motivated to restrict food in order to lose weight (Walsh, 2006). In contrast, people who engage in direct self-injury do consciously aim to hurt themselves (Walsh, 2006).

Within the category of purposeful, direct self-injury, the most controversial referent has been suicidal intent because it is an ephemeral construct that is often extremely difficult to quantify (Freedenthal, 2007). One solution is to view self- 
injurious behaviours on a continuum with the following three anchors: NSSI, ambivalent or ambiguous suicide attempt, and unambiguous suicide attempt (Linehan, Comtois, Brown, Heard, \& Wagner, 2006). Although a theoretically sensible approach, it can be difficult on a practical level to determine where individual acts of self-injury fall on the continuum.

For instance, if a person burns the back of their hand with a cigarette, it is unproblematic to conclude that this behaviour is a type of NSSI, given that cigarette burns cannot cause death and it would be extremely unlikely for anyone to believe that they could. In comparison, overdosing on prescribed medication or illegal drugs is an ambiguous behaviour because it can result in death and is frequently used as a means to attempt or complete suicide. The latest available suicide statistics from Aotearoa New Zealand show that 11\% of people who completed suicide in 2008 poisoned themselves with solids or liquids, compared with only $2 \%$ who cut or pierced themselves (Ministry of Health, 2010). ${ }^{3}$

Other referents (e.g., lethality, tissue damage, function) included in self-injury or self-harm definitions can help to clarify, and support, the parameters of suicidal and non-suicidal behaviours, although this is not always the case. In particular, it can be difficult to classify self-poisoning as suicidal or non-suicidal on the basis of tissue damage and lethality. Tissue damage can result from self-poisoning, but the extent of such damage, if it does occur, can be hard to determine. Likewise, lethality on its own is not always an accurate indicator of intent as some people may hurt themselves with the intent to die but unwittingly use methods that are not sufficiently lethal, whereas others may accidentally complete suicide when they were only intending to self-injure (Brown, Henriques, Sosdjan, \& Beck, 2004; Freedenthal, 2007).

Given the difficulties associated with clarifying suicidal intent specifically in cases of self-poisoning, overdosing and the ingestion of toxic substances are more

\footnotetext{
${ }^{3}$ All of the percentages reported in this chapter have been rounded to zero decimal places.
} 
typically included in studies where the researchers have not discriminated between harmful behaviours on the basis of suicidal intent. In these studies, self-harm is used as an umbrella term to encompass both self-poisoning and self-injury (e.g., Hawton \& Rodham, 2006). However, once again, this is not always the case.

Exceptions to this trend include Gratz's (2003) definition of DSH discussed above, and Nixon, Cloutier, and Jansson's (2008) use of the term non-suicidal selfharm as a superordinate of self-injury (e.g., cutting), overdosing on medication, using drugs or alcohol, and swallowing non-ingestible items or substances. Behavioural measures of NSSI also vary as to whether or not self-poisoning is included; for example, self-poisoning is excluded from the Deliberate Self-harm Inventory (DSHI; Gratz, 2001), while swallowing chemicals, but not drug overdoses, is included in the Inventory of Statements about Self-injury (ISAS; Klonsky \& Olino, 2008).

Verifying the absence of suicidal intent by assessing suicidality indicators, such as lethality and extent of tissue damage (Walsh, 2006), thus clearly has the potential to be misleading. In light of these difficulties, the experiential and empirical evidence that supports distinguishing between behaviours on the basis of suicidal intent warrants further discussion given that I am investigating non-suicidal selfinjurious behaviours in this thesis. In the following section, I will briefly review the literature that demonstrates NSSI is a discrete set of behaviours to suicide attempts, before examining whether given this distinction it is useful to classify NSSI as either a symptom or syndrome of psychopathology.

\subsection{Differentiating non-suicidal self-injury from suicide attempts}

Although the increase of robust empirical research on the differences between non-suicidal and suicidal self-injury is fairly recent, distinguishing between these behaviours is certainly not a novel endeavour. Writing in 1935, Menninger argued that self-injury was a strategy used to avert suicide completion, which "represents a victory...of the life instinct over the death instinct" (p. 466). This corresponds with reports from mental health consumers that self-injury provides respite from overwhelming thoughts and emotions, thus allowing them to continue functioning 
(Alexander \& Clare, 2004; Harris, 2000; Himber, 1994), and Favazza and Conterio's (1988) contention that self-injury is a form of self-help.

Consumer perspectives articulated through personal narratives, research participation, and consultation appear to have informed professional perspectives over time to the point where professional and consumer beliefs about the importance of defining self-injury according to intent have become more aligned. Certainly, there is now substantial experiential and empirical evidence to support distinguishing between NSSI and suicide attempts.

\subsubsection{Consumer perspectives}

Self-injury as self-preservation is powerfully articulated by Kettlewell (1999) in her memoir, Skin Game:

Somewhere over the course of that winter I started thinking about killing myself, though not so much because I wanted to be dead, precisely, as because I yearned for resolution, for escape from the scratching distress of now...I needed to kill something in me, this awful feeling like worms tunnelling along my nerves. So when I discovered the razor blade, cutting, if you'll believe me, was my gesture of hope. That first time, when I was twelve, was like some kind of miracle, a revelation. The blade slipped easily, painlessly through my skin, like a hot knife through butter. As swift and pure as a stroke of lightning, it wrought an absolute and pristine division between before and after. All the chaos, the sound and fury, the uncertainty and confusion and despair - all of it evaporated in an instant, and I was for that moment grounded, coherent, whole. Here is the irreducible self. I drew the line in the sand, marked my body as mine, its flesh and its blood under my command. (p. 57)

Accessing hope and avoiding suicide through self-injury has similarly been

documented by other women who have written about the fundamental role that selfinjurious behaviours have played in securing their survival:

I don't cut myself in an attempt to die! It's not the same thing! The feelings behind my scars are totally different...I look at these lines and shapes as battle scars-necessary sacrifices for the greater good...I really believe had I not cut, I would have died. The feelings I had while doing this were so intense that they would have overpowered me. In my mind it was a choice. Cut or die. It's that easy. (Vega, 2007, p. 141)

Self harm is a survival strategy and frequently represents the least possible damage an individual can get away with. It is an exercise in extreme restraint.

Self harm is about self-worth, self-preservation, lack of choices, coping with the uncopeable, speaking the unspeakable. Self harm gives a physical face to pain that might otherwise extinguish life. If I had not self-injured, I wouldn't be here today. (Pembroke, 1998, p. 20) 
A harm minimisation philosophy is evident within these extracts in that self-injury, when compared to suicide, is presented as the less damaging, and therefore more desirable, option.

In recognition of the paradox that self-injury can sustain life but also cause accidental death, the National Self-harm Network (NSHN) in the United Kingdom published a book entitled: Cutting the risk: Self-harm, self-care $\mathcal{E}$ risk reduction. This book provides explicit instructions about how people should injure themselves to prevent long-term damage or unintended death. When introducing the book, Pembroke (2000) writes that, "Professionals frequently equate recovery with the cessation of self-harm, but that's simplistically shallow and unrealistic. If we do less damage and feel better about ourselves, take greater care of ourselves, then that's a success" (p. 7). Directly challenging professionals who define self-injury as a form of suicidal behaviour, the NSHN frames it as "a valid method of survival, until survival is possible by other means" (p. 6).

\subsubsection{Professional perspectives}

Recent empirical findings support the experiential evidence (that self-injury is not synonymous with attempted suicide) presented by mental health consumers, such as Pembroke (1998), Kettlewell (1999), and Vega (2007). Researchers have identified that while non-suicidal and suicidal self-injurious behaviours do share certain features, there are sufficient, significant differences to warrant distinguishing between these behaviours (Brausch \& Gutierrez, 2010; Whitlock \& Knox, 2007; Wichstrøm, 2009).

Studies investigating the relationship between suicidal behaviours and NSSI routinely demonstrate that not all people who self-injure report past suicidal ideation or attempts (Plener, Libal, Keller, Fegert, \& Muehlenkamp, 2009; Whitlock, Eckenrode, \& Silverman, 2006). In one study, 66\% of university students who had self-injured did not report any past suicidal ideation or attempts (Whitlock et al., 2006), while in another study, $25 \%$ of adolescents had self-injured but only $7 \%$ had attempted suicide (Plener et al., 2009). 
These prevalence rates, however, differ between community and psychiatric samples. Among a group of adolescent inpatients who had self-injured in the previous year, 70\% also reported having attempted suicide (Nock, Joiner, Gordon, Lloyd-Richardson, \& Prinstein, 2006). Although this rate is much higher than reported in community samples, there were still a substantial minority of adolescents (30\%) who had self-injured but had never attempted suicide.

Furthermore, people with a history of self-injury and suicide attempts typically report that they have self-injured significantly more times than they have attempted suicide (Walsh, 2006). Recent ecological momentary assessment research where adolescents and young adults were invited to complete questions, multiple times a day, about any self-destructive thoughts or behaviours they experienced over a two-week period, showed that out of the 1,262 episodes reported, 27\% were categorised as NSSI thoughts, $8 \%$ as NSSI behaviour, and $2 \%$ as suicidal ideation (Nock, Prinstein, \& Sterba, 2009). Additionally, while 87\% of the participants selfinjured without suicidal intent at least once during the course of the study, a minority of $33 \%$ reported suicidal ideation, and none reported any suicide attempts.

Among people who self-injure, non-suicidal self-injurious thoughts and behaviours are not only more prevalent than suicide ideation and attempts, but also are associated with, and predicted by, discrete factors (Wichstrøm, 2009). Prior NSSI and becoming sexually active at younger age have been identified as specific risk factors for NSSI, while conduct problems and suicidal thoughts have been identified as specific risk factors for suicide attempts (Wichstrøm, 2009). Protective factors specific to NSSI and suicide attempts were satisfaction with social support and parental care respectively (Wichstrøm, 2009).

Studies have also identified that suicidal and non-suicidal self-injury serve different functions (Brown, Comtois \& Linehan, 2002; Himber, 1994; Polk \& Liss, 2009), lending further support to distinguishing between these behaviours. Withinand between-person analyses showed that women diagnosed with Borderline Personality Disorder (BPD) more often endorsed using NSSI, as opposed to suicide 
attempts, to express anger, reduce dissociation, and distract themselves (Brown et al., 2002). In comparison, suicide attempts were more often endorsed as a way to improve the lives of others. Self-punishment evidenced a more complicated relationship with NSSI and suicide attempts (Brown et al., 2002). While selfpunishment was more likely to be endorsed in the context of NSSI than suicide attempts between persons, this difference was not identified within persons, suggesting that women with BPD who injure themselves both with and without suicidal intent are likely to do so as a form of self-punishment (Brown et al., 2002).

Although the evidence to date supports the argument that NSSI and suicide attempts are phenomenologically different behaviours, researchers have also found a number of similarities between these behaviours (Brausch \& Gutierrez, 2010; Muehlenkamp \& Gutierrez, 2007; Wichstrøm, 2009). For example, Brown and colleagues (2002) found that particular functions (e.g., emotion relief, interpersonal influence) were similarly endorsed for both NSSI and suicide attempts.

Certain risk factors have also been identified as common to adolescents with history of both NSSI and suicide attempts, and adolescents with a history of NSSI only, but these shared factors tend to vary in intensity between the two groups. Adolescents who have self-injured and attempted suicide report fewer reasons for living, higher rates of suicidal ideation and depressive symptoms, and judge themselves more harshly than adolescents who have only self-injured (Brausch \& Gutierrez, 2010; Dougherty et al., 2009; Muehlenkamp \& Gutierrez, 2007). However, adolescents who have attempted suicide and/or self-injured are at significantly greater risk of suicide completion than adolescents who have never engaged in selfinjurious behaviours (Brausch \& Gutierrez, 2010; Muehlenkamp \& Gutierrez, 2007).

\subsection{Should self-injury be classified as a symptom or a syndrome?}

While non-suicidal and suicidal self-injurious behaviours share certain features, it is clear that they can, and should, be viewed as distinct behaviours. Several researchers who support this distinction have debated whether NSSI should be classified as a symptom of psychopathology or a psychopathological syndrome 
(Favazza \& Rosenthal, 1993; Muehlenkamp, 2005; Pattison \& Kahan, 1983). The evolution of this classification debate is especially pertinent in light of the proposed addition of Non-Suicidal Self-Injury Disorder to the fifth edition of the Diagnostic and Statistical Manual of Mental Disorders (DSM-5; American Psychiatric Association, 2010). Of course, as the following discussion will emphasise, both personal epistemologies and socio-cultural norms once again come into play when defining what counts as a symptom of disorder versus a disorder in and of itself. It is therefore timely to reflect on the propriety and utility of including a self-injury syndrome in the DSM-5, and the impact that such an inclusion may have on nonWestern cultures.

\subsubsection{Self-injury as a symptom}

Traditionally, NSSI among typically developing populations has been conceptualised within academic discourse as a symptom of mental disturbance or disorder, most notably that of BPD. Unfortunately because self-injury is a DSM-IVTR diagnostic criterion for BPD (American Psychiatric Association, 2000), the relationship between self-injurious behaviour and BPD can easily become tautological; self-injurious behaviour is taken as evidence of a BPD diagnosis, and the presence of a BPD diagnosis is then used to explain future episodes of NSSI (Cipani \& Schock, 2007).

Reducing self-injury to the status of a BPD symptom has been criticised by Babiker and Arnold (1997) who declare their professional understanding of selfinjury to be "at odds" (p. 11) with the structuralist epistemology that informs psychiatric research and practice. Self-injury is perceived as one symptom in a "pathology package" (p. 14), which requires immediate intervention and eradication. In contrast, they view self-injury as an embodied signifier of distress, thus emphasising the sociological and communicative aspects of self-injurious behaviour.

Conceptualising self-injury as a sign of suffering, rather than psychopathology, corresponds with how many people who self-injure articulate the 
reasons behind their behaviours (Kettlewell, 1999; Pembroke, 1996a; Vega, 2007). The juxtaposition between professional and consumer understandings of NSSI is a reminder that: "The meanings of symptoms are standardized "truths" in a local cultural system, inasmuch as the groups' categories are projected onto the world, then called natural because they are found there" (Kleinman, 1988, p. 10). When consumers call into question professional conceptualisations of NSSI as indicative of pathology they are questioning these "truths".

Recent research similarly refutes the primacy of self-injury as a symptom of BPD finding that not all people who self-injure meet the criteria for a mental health disorder, and those who do meet the criteria for one or more disorders are a diagnostically heterogeneous group (Hintikka et al., 2009; Nock et al., 2006). Given this heterogeneity, self-injurious behaviours are now more routinely conceptualised within academic discourse as a maladaptive coping mechanism, rather than a symptom of a specific disorder.

Through emphasising the coping function of self-injury, professional perspectives have become more aligned with those of consumer activists, although one important difference remains. Consumer activists tend to dispute the use of the qualifier, maladaptive, preferring instead to endorse self-injury as a legitimate and adaptive strategy for managing overwhelming, and often life-threatening, distress (Pembroke, 1996a). The utility of self-injury thus lies in the functions that these behaviours fulfil, which will be discussed in more detail in Chapters 2 and 3. Certainly from a functional perspective, any behaviour can be understood as an adaptive response to the environment (Sturmey, Ward-Horner, Marroquin, \& Doran, 2007a), thus making the use of the word maladaptive a misnomer within this paradigm.

One way in which consumers challenge the labelling of NSSI as maladaptive is by critiquing the similarities and differences between NSSI and socially acceptable forms of self-injury: 
Socially acceptable forms of self-harm include; excessive smoking, drinking, exercise, liposuction, bikini-line waxing, high heels and body piercing...The socially acceptable range of self-harm clearly does not include; self-cutting, burning, smashing bones and pouring toxic substances over or into our bodies...Some forms of self-harm don't have the social seal of approval. It is denied as an expression of distress. It goes against the pre-occupation with maintaining 'beauty' and achieving some perceived image of 'perfection'. (Pembroke, 1996b, pp. 2-3)

An examination of the parallels between socially acceptable and unacceptable selfinjury (Turp, 2003) serves to destabilise the legitimacy of dominant academic and practitioner conceptualisations of NSSI by focusing on the role of social and cultural norms in defining where the boundaries of psychopathology lie.

\subsubsection{Self-injury as a syndrome}

Building on the argument that self-injury is a maladaptive coping mechanism, several academics at different times have advocated for non-suicidal self-injurious behaviours to be classified as a unique clinical syndrome. Proposed diagnostic labels include: deliberate self-harm syndrome (Pattison \& Kahan, 1983), repetitive selfmutilation syndrome (Favazza \& Rosenthal, 1993), and deliberate self-injury syndrome (Muehlenkamp, 2005). The possible inclusion of NSSI disorder in the fifth edition of the DSM (American Psychiatric Association, 2010), due to be published in 2013, has revived this nosological debate. In the following sections, I discuss the proposed diagnostic criteria for a self-injury syndrome or disorder, whether classifying NSSI within a psychiatric taxonomy is useful or necessary, and finally whether such a classification would impact negatively on non-Western cultures.

\subsubsection{Proposed diagnostic criteria}

In 1983, Pattison and Kahan presented the first comprehensive attempt at describing a deliberate self-harm syndrome, characterised by direct, low lethality self-harmful behaviours. They suggested that the syndrome, which typically begins in late adolescence and comprises of multiple self-injury episodes over a number of years, should be included in the fourth edition of the DSM as an impulse control disorder. Drawing on 56 published case studies, they identified the following six psychological symptoms: an irresistible urge to self-injure, feeling overwhelmed and 
unable to cope, heightened negative emotions, an inability to problem-solve due to cognitive constriction, feeling relieved after self-injury, and depression.

Informed by the work of Pattison and Kahan (1983), Favazza and Rosenthal (1993) similarly proposed that self-injury should be included in the DSM as an impulse disorder, although they used the label, repetitive self-mutilation syndrome. Their suggested diagnostic criteria predominantly focused on the trajectory of selfinjury: (A) a preoccupation with thoughts of self-injury, (B) a persistent inability to resist the urge to self-injure, (C) an escalation of tension immediately prior to selfinjury, and (D) a feeling of relief or gratification during self-injury. Criterion E differentiated NSSI from injuries carried out with conscious suicidal intent or in the context of psychotic experiences or intellectual disabilities. Furthermore, Favazza and Rosenthal advised that self-injury be considered a symptom of disorder until it becomes "an overwhelming preoccupation" (p. 136), at which stage it should be elevated to the status of a syndrome.

More recently, Muehlenkamp (2005) advocated for the inclusion of a Deliberate Self-Injury Syndrome in the DSM. This syndrome bears close resemblance to previous incarnations (Favazza \& Rosenthal, 1993; Pattison \& Kahan, 1983), but expands on some of the earlier criteria. For example, Muehlenkamp's description of the emotional precipitants of NSSI are more detailed: "Preceding the act of selfinjury, there is a psychological experience of increasing tension, anger, anxiety, dysphoria, or general distress, which the person feels he or she cannot escape from or control" (p. 333).

As mentioned earlier, the debate about whether self-injury should be classified as a syndrome has been revived because of the impending publication of the DSM-5. It has been proposed that a diagnosis of NSSI be included in the fifth edition under the category 'Other Disorders' (American Psychiatric Association, 2010). Criterion A of the self-injury disorder specifies the location (i.e., on the body surface, thus excluding overdoses, ingestion of toxic substances or objects), recency (i.e., within the past 12 months), number of episodes (i.e., injured on at least 5 days), 
and severity (i.e., bleeding, bruising, or pain is probable) of the self-injurious behaviour. Lack of suicidal intent is determined through self-report or inferred from the regular use of non-lethal means.

To qualify for the diagnosis, people also need to show evidence of at least two of the four precipitating factors described in Criterion B: (1) negative emotions or cognitions, (2) a preoccupation with self-injury, (3) recurrent urges to self-injure, and (4) anticipation that the self-injury will serve a useful purpose, such as emotional relief. Criterion C stipulates that the "behaviour and its consequences cause clinically significant distress or impairment in interpersonal, academic, or other important areas of functioning", while Criterion D differentiates NSSI from other disorders that include self-injurious behaviours.

\subsubsection{Is taxonomising self-injury within the DSM useful or necessary?}

Those in favour of a self-injury diagnosis argue that classification is useful to ensure a clear, consistent definition of self-injurious behaviours, which in turn will facilitate much-needed research on the etiology, maintenance, and treatment of NSSI (Muehlenkamp, 2005). As a separate diagnosis, NSSI would no longer be conflated with BPD or suicidal behaviours (Muehlenkamp, 2005; Pattison \& Kahan, 1983). Finally, the clinical utility of a self-injury diagnosis for people without any other mental health disorders has been highlighted (Muehlenkamp, 2005).

Academic opposition to the creation of a self-injury syndrome has centred on the limited research available on the causes and course of self-injurious behaviours, the link between self-injury and suicide, and the evidence of high psychiatric comorbidity in people who injure themselves on purpose (Muehlenkamp, 2005). Given the now well-established differences between self-injury and suicide attempts discussed above and the fact that comorbidity does not preclude the existence or diagnosis of other disorders (Muehlenkamp, 2005), I will briefly speculate about the possible consequences of creating a new diagnosis in the absence of sufficient research. 
The paucity of longitudinal research into how self-injurious behaviours manifest and change over time (Muehlenkamp, 2005; Prinstein, 2008) is problematic as it could result in the unnecessary pathologising of what may be predominantly adolescence-limited behaviours (cf. Moffitt, 1993). One unintended consequence (Wykes \& Callard, 2010) of a self-injury diagnosis could be that young people who experiment with self-injury are 'encouraged' to adopt the identity of a self-injurer through being diagnosed as such. Furthermore, an NSSI diagnosis could constrain our developing understanding of how to treat and ultimately prevent more chronic self-injurious behaviour (Wykes \& Callard, 2010). Of course, the dilemma is that there is likely insufficient research to warrant the creation of a self-injury diagnosis, but without a fixed definition of NSSI that would accompany a diagnosis, it is more difficult to develop a robust evidence base about self-injury (Muehlenkamp, 2005).

The proposal to classify self-injury within the structuralist bounds of the DSM also runs counter to the increasingly functionalist approach taken by self-injury researchers. The importance of functionalism in understanding why people selfinjure will be discussed in more detail in Chapter 4, but it is useful to note here that one of the key differences between structuralists and functionalists is that the former maintain the causes of behaviour lie within people, whereas the latter maintain the causes of behaviour lie within the environment (Sturmey et al., 2007a).

Diagnoses therefore can be counter-productive in dealing with problem behaviours, particularly if they prevent a thorough assessment of the contingencies maintaining the behaviour (Cipani \& Schock, 2007). This can lead to the tautology described earlier in this chapter where certain behaviours are interpreted as evidence of a disorder, and the disorder is then used to justify the presence of the behaviours (Cipani \& Schock, 2007). The resulting danger is that practitioners fail to appreciate, or remain cognisant of, the influence of social and cultural norms on definitions and manifestations of self-injury.

It is imperative to consider the impact that a self-injury diagnosis may have on people who self-injure and qualify for the diagnosis, as well as those who self- 
injure but do not qualify for the diagnosis. While it is likely that some consumers will find a self-injury diagnosis validating, others may feel stigmatised by being labelled with NSSI disorder. Conversely, those people who do not meet the threshold required for a diagnosis may be denied access to mental health support or feel that their distress has been minimised.

\subsubsection{How would a NSSI disorder impact on non-Western cultures?}

Although the DSM is a classification system based on Western conceptualisations of mental distress and disorder, it is increasingly being used around the world to guide diagnosis and treatment (Watters, 2010; Wykes \& Callard, 2010). Given that socio-cultural norms determine what meanings are ascribed to selfinjurious behaviours, it is important to consider how the proposed addition of NSSI disorder to the DSM-5 (American Psychiatric Association, 2010) will shape nonWestern cultures' understanding and treatment of these behaviours.

In Crazy like us: The globalization of the American psyche, Watters (2010) argues that American constructions of mental illness are not only rapidly changing the way in which psychological distress is perceived in non-Western societies, but also the way in which this distress is experienced. He contends that mental health practitioners who promote the use of DSM criteria in countries that do not subscribe to Western models of health and wellbeing unintentionally become vectors for the disorders they are attempting to treat.

To support his argument, Watters (2010) refers to the work of Shorter (1987) who maintains that each culture has a "symptom pool" (p. 69) that changes over time; people communicate psychological distress through a restricted set of symptoms that are drawn from this pool. Particular behaviours become legitimised as culturally appropriate signifiers of disorder through their inclusion in the symptom pool (Shorter, 1987). Given the global power exerted by the DSM, the socalled "bible" of mental disorders (Watters, 2010, p. 3), a NSSI diagnosis may influence the way in which other cultures manifest distress and inadvertently increase the prevalence of self-injurious behaviours. 


\section{DEFINING SELF-INJURY FOR THE PURPOSE OF THIS THESIS}

This chapter has highlighted the importance of considering how both sociocultural norms and personal epistemologies shape our understanding of what counts as non-suicidal self-injurious behaviours. As a trainee Clinical Psychologist, I value structuralist, functionalist, and experiential ways of knowing, and believe that all of these epistemologies can, and should, inform research and practice. By using a mixed-methods design (interviews and surveys) in this thesis, I have attempted to blend these three approaches and balance both experiential and empirical knowledge.

The following definition of self-injury, used in this thesis, is a combination of the definitions presented in Table 1 and is informed by the debates discussed earlier in this chapter: NSSI is the purposeful, direct, and self-inflicted destruction or alteration of one's body tissue without suicidal intent and for reasons not socially sanctioned. It is distinct from major or stereotypic self-injury conducted in the context of psychotic or developmental disorders respectively (Favazza, 2011). Furthermore, given the difficulties in clarifying suicidal intent in cases of selfpoisoning (see pp. 13-14), overdosing and the ingestion of toxic substances are also excluded from my definition of NSSI. Finally, my understanding of self-injury is consistent with current clinical guidelines (National Institute for Clinical Excellence, 2004; Penrose-Wall, Farris, \& Berkery, 2005); self-injury is a behaviour, not a disorder, which fulfils an array of different functions.

In the following two chapters, I review what leads people to injure themselves on purpose in the absence of suicidal intent and why they may become reliant on these behaviours to cope with distress, before describing the three studies I conducted to investigate whether self-injury functions primarily as an experientially avoidant behaviour within Aotearoa New Zealand. 


\section{CHAPTER TWO}

\section{Why do people self-injure?}

"I know why I self-injure. I do it at times of extreme emotion: anger, self-hatred, stress, grief and guilt. I do it to punish myself. When I feel I am losing control, I reach for a razor and prove to myself that I can, at least, have control over my body." (Ross, 1996, p. 13)

\section{INTRODUCTION}

Publications on self-injury have more than tripled within the last decade (Nock, 2010), which has contributed to an increased awareness of the incidence and prevalence of these behaviours, especially among young people (Ross, Heath, \& Toste, 2009). The burgeoning evidence base addressing self-injurious behaviours can be divided into two broad domains: studies that investigate the risk factors and correlates of NSSI (e.g., Gratz, Conrad, \& Roemer, 2002; Hankin \& Abela, 2011; Wichstrøm, 2009), and studies that investigate why people self-injure (e.g., Klonsky \& Glenn, 2009; Lloyd-Richardson, Perrine, Dierker, \& Kelley, 2007; Nock \& Prinstein, 2004). Both streams of research are vital to developing a comprehensive understanding of what factors increase the likelihood that people will self-injure, and, once they begin self-injuring, how their self-injurious behaviours are reinforced and maintained over time.

The studies for this thesis fall into the latter domain; my research is concerned with why people living in Aotearoa New Zealand self-injure and seeks to further clarify the behavioural functions of NSSI. In particular, I am interested in whether self-injury, carried out within the context of Aotearoa New Zealand, can be understood primarily as a form of experiential avoidance, a behavioural process that reflects people's unwillingness to tolerate aversive, intrapersonal experiences (Hayes, Wilson, Gifford, Follette, \& Strosahl, 1996). Extant research suggests that affect regulation is the primary function of NSSI (e.g., Klonsky, 2007; Klonsky \& Glenn, 2009; Nock \& Prinstein, 2004), but the broader, overarching concept of experiential avoidance, which includes but is not limited to affect regulation, has 
seldom been examined in the international literature and has never been empirically investigated within Aotearoa New Zealand.

Although I am not focussing on the risk factors and correlates of NSSI, it would be remiss to omit a discussion of these characteristics because they provide the context, through specifying who is more likely to self-injure, in which to situate my empirical work for this thesis. With this in mind, I review the research conducted on the individual, psychological, and environmental factors that predict, or are associated with, self-injury, before focusing in more detail on what we currently know about why people are motivated to hurt themselves on purpose. ${ }^{4}$

\section{HOW PREVALENT IS SELF-INJURY?}

Before discussing self-injury correlates, risk factors, and motivations, it is essential to briefly summarise how many people self-injure and when they begin engaging in these behaviours. Unfortunately, precise prevalence rates of NSSI in clinical and community populations are unknown; figures tend to vary considerably across studies due to inconsistent operational definitions and modes of measurement (Ross et al., 2009). Evidence from population-based surveys suggests that $2 \%$ to $6 \%$ of adults have self-injured without suicidal intent in their lifetimes (Bebbington et al., 2010; Briere \& Gil, 1998; Klonsky, 2011), although the rate of lifetime NSSI was as high as $17 \%$ in a random sample of university students (Whitlock et al., 2006). The identical prevalence rate of $17 \%$ was reported in a population-based study of adolescents and young adults (Nixon et al., 2008). Much higher prevalence rates of $36 \%$ to $63 \%$ have been observed among clinical populations (Claes, Vandereycken, \& Vertommen, 2007; Swenson, Spirito, Dyl, Kittler, \& Hunt, 2008; Weismoore \& Esposito-Smythers, 2010). On average, people begin self-injuring between the ages of 11 to 15 (Hankin \& Abela, 2011; Nixon, Cloutier, \& Aggarwal, 2002; Yates, Carlson, \& Egeland, 2008).

\footnotetext{
${ }^{4}$ It should be noted that my review about why people are motivated to self-injure is similarly structured to those presented by other authors (e.g., Klonsky, 2007).
} 


\section{RISK FACTORS AND CORRELATES OF SELF-INJURY}

The equifinality of NSSI is evident in the wide range of individual, psychological, and environmental characteristics that are associated with selfinjurious behaviours. Individual factors that have been implicated in the development of NSSI include gender, ethnicity, and sexuality. Psychological factors, such as mental health disorders, suicidality, temperament, and alexithymia are also thought to play an aetiological role in self-injury, along with environmental characteristics, including experiences of maltreatment during childhood and peer victimisation.

\subsection{Individual characteristics}

\subsubsection{Gender}

Non-suicidal self-injury traditionally has been considered to be a gendered phenomenon, with more females than males engaging in NSSI, until recent evidence from community-based studies suggested that this gender ratio may be a sampling artefact. Much of the earlier research on self-injury focused on clinical populations, which were comprised of a disproportionate number of women (Whitlock et al., 2006). Within these populations, people diagnosed with BPD have been of specific interest to researchers because self-injury is one of the $D S M-I V$-TR criteria for the disorder (American Psychiatric Association, 2000). Since women are purportedly three times more likely to be diagnosed with BPD than men (Skodol \& Bender, 2003), the research focus on self-injury within BPD populations has arguably supported a sampling bias in the study of NSSI.

Preliminary evidence has also shown that there are gender differences in the forms of NSSI used by females and males, with females being more likely to cut themselves and males being more likely to burn themselves (Andover et al., 2010; Whitlock et al., 2006). Studies that have focussed exclusively on particular forms of self-injury (e.g., cutting) thus may have drawn premature conclusions about gender differences in NSSI, as a result of gendered preferences for certain forms of selfinjury. 
Although it is possible that being female is not necessarily a risk factor for NSSI, the evidence to support gender equivalency in NSSI prevalence rates is far from conclusive. Several cross-sectional studies of community-based adolescents and young adults have reported that females are significantly more likely to selfinjure than males (Hoff \& Muehlenkamp, 2009; Nixon et al., 2008; Plener et al., 2009; Ross \& Heath, 2002), while others have failed to find any significant gender differences in NSSI prevalence (Andover et al., 2010; Claes, Houben, et al., 2010; Gratz et al., 2002; Muehlenkamp \& Gutierrez, 2004). In a prospective study with Norwegian adolescents conducted over five years, females were twice as likely to self-injure as males and being female was a risk factor for NSSI (Wichstrøm, 2009).

One of the reasons for these conflicting results could be due to the age group being assessed for NSSI, as the ratio of female to male self-injury may vary according to stages in the life cycle (Hawton \& Harriss, 2008). Data collected on hospital presentations for DSH over 10 years showed that overall, females were one and a half times more likely than males to present to hospital following an act of DSH, but this general ratio masked sizeable differences between age groups. Females were eight times more likely to self-harm than males in the 10-14 year old age group and approximately three times more likely to self-harm in the 15-19 year old age group. This female to male ratio in DSH rates then decreased considerably from the age of 20 until age 50, when males engaged in higher rates of DSH than women (Hawton \& Harriss, 2008).

Although caution is required when extrapolating these results to gender differences in NSSI - this study relied exclusively on hospital admissions for selfharm and did not distinguish between different types of self-harm on the basis of intent-it is possible that a similar, age-related trend may be observable in female to male ratios of NSSI. An archival study of self-reported, lifetime prevalence rates of NSSI among secondary school students over 5 years showed that while there were no gender differences in NSSI rates for the first three years of secondary school, selfinjury was more prevalent amongst females than males during the last two years of 
school (Muehlenkamp, Williams, Gutierrez, \& Claes, 2009). Population-based research on the prevalence of NSSI throughout the life cycle is needed to determine whether self-injurious behaviours are actually more common among females than males.

\subsubsection{Ethnicity}

Sampling biases have also precluded a thorough investigation of the role of ethnicity in the development of NSSI. Historically, self-injury has been conceptualised as a largely Caucasian phenomenon, but this conclusion is based on a body of literature where the overwhelming majority of participants are white (Gratz, 2006; Shaw, 2002). The dearth of evidence into the impact of ethnicity on developmental trajectories of NSSI is especially problematic considering that, as I discussed in Chapter 1, what counts as pathological self-injury is culturally bound (Favazza, 2011).

The few studies that have recruited ethnically diverse samples have presented conflicting results as to whether Caucasians are more likely than other ethnicities to engage in NSSI. No significant differences in the lifetime prevalence of NSSI were found in an ethnically diverse sample (i.e., 35\% Caucasian, 37\% African American, 16\% multi-ethnic etc.) of secondary school students (Brausch \& Gutierrez, 2010). However, in an inpatient sample with approximately even numbers of Caucasian and African American adolescents and a small percentage of other ethnicities (e.g., Hispanic, Native American), young people from ethnic minorities were less likely to have engaged in NSSI, but more likely to have attempted suicide (Boxer, 2010).

In Aotearoa New Zealand, young Māori people are two to three times more likely to complete suicide than non-Māori youth (Beautrais \& Fergusson, 2006), but the difference in NSSI prevalence between ethnicities is unclear. More Māori (25\%) than Pākehā (19\%) youth reported DSH in a recent national survey of health and wellbeing among young New Zealanders (Fortune et al., 2010), but the survey question referred to engaging in self-harm or any actions that may have resulted in 
injury or death, rendering it impossible to separate out non-suicidal from suicidal self-injury.

Given the importance of culture in delineating the taxonomical boundaries of NSSI, studies conducted by Māori researchers with tangata whaiora ${ }^{5}$ and community-based Māori populations are essential to determine the extent of NSSI among Māori people. Utilising questions about self-injury, which are developed within a Western paradigm of NSSI, to determine how many Māori self-injure and the factors that place Māori at risk of self-injuring, makes it less likely that an indepth understanding of this phenomenon among Māori will result.

Furthermore, significant cross-cultural differences may be overlooked because Western-based questions about NSSI do not take into account contextual factors that are specific to Māori, or other indigenous peoples and minority groups. For example, it has been argued that the higher suicide rate among Māori is a result of the enduring consequences of colonisation, such as the loss of cultural identity and connectedness to Māori culture (Lawson-Te Aho \& Liu, 2010). Any research that examines NSSI among Māori needs to take the complex dynamics of postcolonialism into account (Wilson, 1999).

Aotearoa New Zealand is also home to a number of other ethnic groups, including Pacific peoples and Asians, whose experiences of NSSI are yet to be examined. Unique socio-cultural factors will undoubtedly affect NSSI rates in these communities. For instance, a recent review found that South Asian women living in the United Kingdom are significantly more likely to self-harm than Caucasian women, but the authors did not discriminate between suicidal and non-suicidal selfharm (Husain, Waheed, \& Husain, 2006). Factors implicated in the high rates of DSH among these women included racism, domestic violence, forced marriage, and the pressure to abide by their families' or communities' beliefs about izzat, defined as

\footnotetext{
5 Tangata whaiora is the Māori term for mental health consumers.
} 
"family or personal honour/respect, or as status and prestige in the eyes of the community" (Chew-Graham, Bashir, Chantler, Burman, \& Batsleer, 2002, p. 342).

\subsubsection{Sexuality}

Other individual characteristics that have been associated with higher rates of NSSI are sexual orientation and same-sex attraction. In one study, university students who were uncertain about their sexual orientation or who identified as bisexual were more likely to report self-injury than heterosexual students (Whitlock et al., 2006). Concerns about sexual orientation were also associated with DSH in a community sample of adolescents, but given that the definition of DSH included suicide attempts, the specific role of NSSI in this association is unclear ( $\mathrm{O}^{\prime}$ Connor, Rasmussen, Miles, \& Hawton, 2009). In another study that examined the relationship between NSSI and sexual orientation, adolescents who identified as homosexual or bisexual were significantly more likely to have self-injured than heterosexual adolescents (Deliberto \& Nock, 2008).

Within Aotearoa New Zealand, the impact of sexual attraction on self-harm has been examined in two research studies. Same-sex attraction or being attracted to both sexes placed male and female secondary school students at significantly higher risk of engaging in self-harm behaviours (Lucassen et al., 2011). Adult New Zealanders who reported same-sex attraction were similarly found to be at risk of self-harm (Skegg, Nada-Raja, Dickson, Paul, \& Williams, 2003). Unfortunately NSSI was conflated with DSH in both of these studies making it impossible to generalise these results to people who self-injure without suicidal intent.

Further research is needed before it can be conclusively determined whether non-heterosexual orientation and/or attraction are risk factors for NSSI. Such research should take advantage of increasingly sophisticated understandings of sexuality. For instance, in their longitudinal study of adolescent health, SavinWilliams and Ream (2007) assert that sexual orientation should be measured as a dimensional, rather than categorical, construct and define sexuality on the basis of attraction, behaviour, and identity. 


\subsection{Psychological characteristics}

\subsubsection{Mental health disorders}

Although NSSI is listed as a symptom of BPD in the DSM-IV-TR (American Psychiatric Association, 2000), it has been associated with a range of other mental health conditions, including disordered eating (Ross et al., 2009; Whitlock et al., 2006), depression, and anxiety disorders (Hintikka et al., 2009; Hoff \& Muehlenkamp, 2009). In a Finnish study, adolescent girls who cut themselves were more than three times as likely to have a mental health diagnosis than age-matched controls (Hintikka et al., 2009), although given that the study design was crosssectional, it is not possible to determine whether the high rate of mental disorders in this sample was a causal factor in the development of NSSI. Furthermore, since the type of NSSI was limited to cutting, such high rates of mental disorders may not be observed among people who use other forms of self-injury.

Preliminary support for the suggestion that different forms of NSSI may be associated with different types of mental disorders has been provided by Andover, Pepper, Ryabchenko, Orrico, and Gibb (2005). University students who had cut themselves reported more anxiety than those who used other forms of self-injury, but the rates of depressive symptoms were similar in both groups. Further analyses by Andover and colleagues identified that although the students who had selfinjured were significantly more likely to report symptoms of depression and anxiety compared to a control group, these differences were no longer significant after controlling for BPD symptoms even though none of their participants met the threshold for a diagnosis of BPD.

The nature of the relationships between mental health disorders and NSSI, however, will most likely differ according to the severity of psychopathology experienced by participants. For example, in a prospective research study conducted over 10 years, Zanarini, Laudate, Frankenburg, Reich, and Fitzmaurice (2011) found that major depression was a significant predictor of NSSI in people diagnosed with BPD. Additional studies are needed to tease out the contribution of various forms of 
psychopathology to NSSI and how comorbid disorders interact to influence the manifestation of self-injurious behaviour.

It is also probable that any interactions between mental disorders and NSSI will change over time both within and between individuals. In a study of secondary school students using archival data gathered over five years, Muehlenkamp et al. (2009) found that as females' rates of NSSI increased over time, their symptoms of depression decreased. Given that NSSI is thought to function primarily as an affect regulation strategy (Klonsky, 2009), one possible explanation for this pattern is that self-injury was increasingly used to reduce depressive symptoms. It is unclear why this pattern was not observed in males, but Muehlenkamp et al. (2009) hypothesise their ability to identify statistically significant changes may have been impeded by the small number of male participants.

It is important to keep in mind that although a range of mental health disorders have been identified as correlates or predictors of NSSI, the experience of psychopathology is not a prerequisite for engaging in self-injurious behaviours. In one study, $21 \%$ of the adolescents who had cut themselves did not meet the criteria for an Axis-I disorder as measured by the Structured Clinical Interview for DSM-IVTR (Hintikka et al., 2009). However, the authors acknowledge that these adolescents may meet the criteria for the Axis-II disorder of BPD later in life.

\subsubsection{Suicidality}

Owing to the historical conflation of non-suicidal and suicidal self-injury, which I discussed in-depth in Chapter 1 (see pp. 14-18), research on the role of suicidality (i.e., suicidal ideation and attempts) in NSSI is limited. In Chapter 1, I cited experiential and empirical evidence in support of a distinction between NSSI and suicide attempts. In the current section, I review this empirical evidence in more detail to further unpack the complex relationship between non-suicidal and suicidal self-injury.

Repetitive DSH, irrespective of suicidal intent, has been identified as a suicide risk factor for both females and males (Zahl \& Hawton, 2004), but the unique 
contribution of NSSI to suicide completion is unclear. To address this, researchers have started to compare people who have self-injured or attempted suicide, with those who have self-injured and attempted suicide, in order to identify correlates and risk factors specific to each group. In particular, the impact of suicidal ideation and past suicide attempts on NSSI has been examined.

A comparison of a group of secondary school students who had self-injured to a group who had attempted suicide found no difference in suicidal ideation between the two groups (Muehlenkamp \& Gutierrez, 2004). However, when secondary school students who had self-injured and attempted suicide were compared to those who had only engaged in self-injury, those with a history of NSSI and suicide attempt(s) reported significantly higher levels of suicidal ideation than those who with a history of NSSI only (Brausch \& Gutierrez, 2010; Muehlenkamp \& Gutierrez, 2007).

Slightly different results were obtained in a sample of adolescent outpatients (Jacobson, Muehlenkamp, Miller, \& Turner, 2008). In this study, when adolescents with a history of NSSI only were compared to adolescents who had engaged in NSSI plus attempted suicide, and adolescents who had only attempted suicide, those who had only engaged in self-injury had significantly lower rates of suicidal ideation. The group of adolescents who had self-injured and attempted suicide, and the group who had only attempted suicide but had not self-injured, did not differ significantly from each other on suicidal ideation.

Evidence in support of a relationship between NSSI and suicide attempts has been mixed. No association between NSSI episodes and suicide attempts was found in a group of inpatient adolescents (Nock et al., 2006), but in a group of adult inpatients, those with a history of NSSI were more likely to have attempted suicide than those without a history of NSSI (Andover \& Gibb, 2010). Moreover, Andover and Gibb (2010) found that suicide attempts were positively associated with NSSI frequency. Although there was no difference between current suicidal ideation versus NSSI frequency and history in predicting suicide attempts, the number of 
times people had self-injured was a stronger predictor of suicide attempts than hopelessness, depression, and BPD symptoms. Additionally, the presence of an NSSI history was a significant predictor of attempted suicide regardless of how many times people had self-injured.

The predictive value of NSSI has been examined not only for suicide attempts and ideation, but also for plans and gestures (Whitlock \& Knox, 2007). Among people who reported NSSI and suicidality, NSSI strongly predicted all forms of suicidality (i.e., ideation, plans, gestures, and attempts). However, these results rely on the assumption that NSSI precedes or occurs simultaneously with suicidality (Whitlock \& Knox, 2007), which may not be the case. Evidence from a prospective study of Norwegian adolescents showed that while previous suicide attempts predicted future NSSI, previous NSSI did not predict future suicide attempts, suggesting that NSSI is not a risk factor for suicide attempts but, rather, prior suicide attempts place adolescents at risk of engaging in NSSI (Wichstrøm, 2009).

Given that self-injuring without suicidal intent does appear to place some people at risk of attempting suicide, it is important to consider why this may be the case. Joiner (2005) argues that one way people acquire the ability to complete suicide is through practising self-injury until these behaviours become habitual and unthreatening. Cross-sectional studies that have identified a positive association between the frequency of NSSI and suicide attempts (Andover \& Gibb, 2010; Whitlock \& Knox, 2007) provide preliminary support for Joiner's theory, but such support is tempered by research that has failed to find evidence of this association (Nock et al., 2006).

If people do become habituated to the prospect of suicide through self-injury, what prompts the shift from one to the other? One possibility is that when people who use self-injury as a coping strategy surpass a particular threshold of distress, which cannot be alleviated by other means (including self-injury), they may attempt suicide (Whitlock \& Knox, 2007). More specifically, the connection between suicidal and non-suicidal self-injury may lie in the shared, unresolved distress that motivates 
these behaviours (Whitlock et al., 2006). Prospective, longitudinal research is clearly needed to test Joiner's (2005) habituation hypothesis.

\subsubsection{Temperament}

Specific temperament traits, such as impulsivity and emotional reactivity, have been identified as individual risk factors for NSSI. As mentioned earlier, several researchers have suggested that NSSI be classified as an impulse control disorder (Favazza \& Rosenthal, 1993; Pattison \& Kahan, 1983), but findings about the relationship between NSSI and impulsivity have been mixed. Utilising child and parent reports of temperament dimensions, Baetens, Claes, Willem, Muehlenkamp, and Bijttebier (2011) found that lack of effortful control (i.e., a decreased capacity for attention and behaviour regulation) was one of the most robust predictors of NSSI in a group of secondary school students.

However, others maintain that there is insufficient evidence to support the role of impulsivity in NSSI, in part, because it is a heterogeneous construct that has been operationalised and measured differently across studies, resulting in conflicting findings (Glenn \& Klonsky, 2010a). For example, Herpertz (1995) categorised NSSI episodes as impulsive if people evidenced the following three criteria: a lack of premeditation and consideration of consequences, acting on the decision to selfinjure within minutes, and an urge to self-injure, whereas other studies have assessed the association between impulsivity and self-injury through assorted validated measures (e.g., Jutengren, Kerr, \& Stattin, 2011; MacLaren \& Best, 2010).

Two studies that utilised multiple methods (e.g., self-report and laboratorybased tasks) to measure impulsivity sought to clarify whether people who self-injure are more impulsive than those who do not self-injure (Glenn \& Klonsky, 2010a; Janis \& Nock, 2009). Both studies found the same pattern of results: people who had selfinjured reported greater impulsivity than people who did not have a history of NSSI, but these differences were not evident in performance-based measures of impulsivity. 
One possible explanation for these inconsistencies is that only particular negative emotions trigger impulsivity in people who self-injure; such emotions would therefore need to be induced in a laboratory setting to capture the relationship between NSSI and impulsivity (Glenn \& Klonsky, 2010a; Janis \& Nock, 2009). It is also essential to examine this relationship over time in order to disentangle whether impulsivity is a cause or effect of NSSI (Janis \& Nock, 2009).

Another temperamental risk factor for self-injury that is garnering increased attention from researchers is whether people are predisposed to experience, and react to, emotions in specific ways (Nock, Wedig, Holmberg, \& Hooley, 2008). This focus is congruent with the conceptualisation of NSSI as an emotion regulation strategy and draws on research conducted with people diagnosed with BPD (Rosenthal et al., 2008). Emotional vulnerability has been proposed as a core mechanism underlying the development of BPD; people who are emotionally vulnerable are characterised by "high sensitivity to emotional stimuli, emotional intensity, and slow return to emotional baseline" (Linehan, 1993b, p. 43). Emotion reactivity is comparable to emotion vulnerability in that it has been theorised to include three components: (1) how emotionally sensitive people are to stimuli, (2) how intensely they experience emotions, and (3) how long emotions persist for following their initiation (Nock et al., 2008).

Due to the absence of a comprehensive, self-report measure of emotion reactivity, Nock et al. (2008) recently developed the Emotion Reactivity Scale to assess emotional sensitivity, arousal/intensity, and persistence. Preliminary evidence demonstrated the validity and reliability of the scale, and people who had recently self-injured reported themselves as significantly more emotionally reactive compared to people who had not self-injured. Moreover, the relationship between psychopathology and NSSI was mediated by emotion reactivity. Unfortunately, the cross-sectional nature of the data precluded any definitive conclusions about whether emotion reactivity drives people with mental health disorders to engage in NSSI (Nock et al., 2008). 


\subsubsection{Alexithymia}

Researchers are not only interested in the way in which people who selfinjure react to emotions, but also how they understand and articulate these emotions. Alexithymia, which has been associated with NSSI (Paivio \& McCulloch, 2004; Zlotnick et al., 1996), is a term that encapsulates a set of difficulties with identifying and discriminating between various emotions, and with verbally communicating those emotions (Kooiman, Spinhoven, \& Trijsburg, 2002). Little is known about whether alexithymia is causally related to self-injury as the research conducted to date has been cross-sectional. Certainly, more work is needed on whether specific groups of people who self-injure are more likely to exhibit alexithymic tendencies, how the different components of alexithymia contribute to the manifestation of self-injury, and whether alexithymia is a causal factor for NSSI.

For instance, Oyefeso, Brown, Chiang, and Clancy (2008) found that people addicted to opiates who had self-injured reported significantly more difficulty identifying their emotions than people addicted to opiates who had not self-injured. However, these two groups did not differ on their self-reported ability to describe how they were feeling. There is also preliminary evidence to suggest that people who have difficulty identifying and expressing their emotions may turn to selfinjury following traumatic experiences. In a cross-sectional study with female university students, alexithymia fully mediated the relationship between child maltreatment (i.e. physical and emotional abuse and neglect) and self-injurious behaviours (Paivio \& McCulloch, 2004).

\subsection{Environmental characteristics}

\subsubsection{Child maltreatment}

The experience of child maltreatment has been proposed as a causal factor in the aetiology of NSSI (Yates, 2004), yet the evidence to support this contention remains conflicted. A group of university students who had been abused were significantly more likely to have self-injured than those without an abuse history (Muehlenkamp, Kerr, Bradley, \& Adams Larsen, 2010), but neither childhood 
physical or sexual abuse was associated with NSSI in a sample of adolescent inpatients (Weismoore \& Esposito-Smythers, 2010). However, in another adolescent sample, self-injuring without suicidal intent in the previous 12 months was significantly related to physical neglect, emotional abuse, and sexual abuse (Glassman, Weierich, Hooley, Deliberto, \& Nock, 2007). Within Aotearoa New Zealand, childhood sexual abuse has been identified as a risk factor for self-injury among depressed adults (Joyce et al., 2006).

The type of maltreatment is one factor which may influence the nature of the relationship between NSSI and abuse history. Indeed, Yates et al. (2008) reported that community-based adults who had been sexually abused as children were almost 10 times as likely to engage in recurrent self-injury (three or more episodes) but those who had been physically abused were more than seven times as likely to engage in intermittent self-injury (one to two episodes). The finding that the type of abuse experienced may impact on the frequency of NSSI was supported in a study by Whitlock et al. (2006). In their sample of university students, single versus repeated incidents of NSSI were differentially associated with particular types of abuse. When compared to students who had never self-injured, those who had selfinjured once were more likely to report past emotional abuse, while those who had self-injured repeatedly were more likely to report past emotional and sexual abuse.

Child sexual abuse (CSA) has arguably received the most attention in relation to NSSI, potentially as a result of the high prevalence of both CSA and self-injury in populations with BPD (Lieb, Zanarini, Schmahl, Linehan, \& Bohus, 2004). Indeed, CSA was a significant predictor of NSSI in people with BPD in a longitudinal 10-year study (Zanarini et al., 2011). However, meta-analytic and review studies have not supported such definitive results.

A recent meta-analysis of 43 studies demonstrated a small relationship between CSA and self-injury, with CSA explaining at most $5 \%$ of the variance in NSSI aetiology (Klonsky \& Moyer, 2008). Furthermore, this relationship became more tenuous when other psychological and environmental risk factors (e.g., 
dissociation, family functioning) were controlled for. The authors concluded that (1) CSA is either a proxy risk factor for NSSI in that the relationship between CSA and NSSI exists because of shared psychological risk factors or (2) CSA may mediate the relationship between other risk factors and NSSI. This conclusion is partially supported by a review that identified CSA as a non-specific risk factor for engaging in self-harm irrespective of suicidal intent (Maniglio, 2011).

In light of the conflicting evidence regarding the contribution of different types of childhood maltreatment to the development of NSSI, it is important for researchers to examine factors that may mediate or moderate the relationships between types of maltreatment and self-injury to shed light on these relationships. For example, when Weierich and Nock (2008) examined the impact of CSA and physical and emotional abuse/neglect on adolescent self-injury, they found that NSSI was only significantly related to CSA. Further analyses revealed that this relationship was fully mediated by Post-traumatic Stress Disorder (PTSD) symptom clusters of avoidance/numbing and re-experiencing. Shenk, Noll, and Cassarly (2010) similarly found that posttraumatic stress symptoms fully mediated the relationship between child abuse and NSSI among female adolescents.

\subsubsection{Peer victimisation}

Another factor that has been implicated in the development of NSSI among young people is bullying, but it has not been extensively investigated. Research from Aotearoa New Zealand showed that reports of bullying were greater among secondary school students who had self-harmed than those who had not selfharmed, but did this study did not distinguish between NSSI and DSH (Garisch \& Wilson, 2010). Peer victimisation was identified as a risk factor for NSSI in a Swedish study where secondary school students were interviewed at two time points, 12 months apart (Jutengren et al., 2011), but did not predict NSSI among a community group of adolescents in the United States (Heilbron \& Prinstein, 2010).

Indeed, the relationship between different types of peer victimisation and NSSI appears complex. An investigation of peer-nominated overt (e.g., hitting) and 
relational (e.g., exclusion, spreading rumours) victimisation in community-based adolescents showed that female victims of overt bullying were less likely to report having self-injured, while male victims of overt bullying were more likely to report having self-injured (Heilbron \& Prinstein, 2010). In a sample of female, adolescent inpatients, the relationship between peer relational difficulties (i.e., overt victimisation, relational victimisation, and negative friendship interactions) and NSSI was mediated by emotional dysregulation (Adrian, Zeman, Erdley, Lisa, \& Sim, 2011). Once again, further research is needed to replicate and clarify these findings.

\subsection{Summary and limitations}

Given the diversity of the individual, psychological, and environmental correlates and risk factors for self-injurious behaviour, it is worth investigating whether there are particular typologies of NSSI. In two recent studies with university students, researchers have attempted to clarify whether people who selfinjure can be divided into clinically distinct subgroups (Klonsky \& Olino, 2008; Whitlock, Muehlenkamp, \& Eckenrode, 2008). Utilising latent class analyses, both studies provided evidence for distinct typologies of NSSI, which were based on factors such as the form, frequency, contextual features, and functions of the selfinjurious behaviour.

Although identifying clinically relevant distinctions between subgroups of people who self-injure is a promising start to unpacking the complexity and heterogeneity of NSSI, there are still many unresolved questions that need to be addressed. When considered in isolation, the majority of the risk factors that have been empirically tested do not only contribute to the aetiology of NSSI, but are predictive of numerous psychopathological outcomes (Nock, 2010). Little research has been conducted on the interaction of multiple risk factors to determine how these interactions influence the development and maintenance of NSSI (Gratz, 2003).

Furthermore, Fliege et al. (2009) have disputed whether many of the characteristics identified in the literature as predictors of NSSI are, in fact, risk 
factors for self-injury. After reviewing 59 studies on NSSI, they concluded that many of the risk factors cannot be characterised as such because NSSI is typically investigated cross-sectionally rather than longitudinally, there is insufficient evidence that the risk factors were present before the self-injurious behaviour began, and none of the studies prospectively investigated new incidents of NSSI. Certainly, Glenn and Klonsky (2011) identified that several well-established correlates of NSSI failed to prospectively predict self-injurious behaviours.

In sum, identifying the contribution of varied risk factors and correlates to the development of NSSI is essential to create effective prevention programmes, and much more work is needed in this area. However, even if a solid, comprehensive evidence base about these factors did exist, this information would still be of limited treatment utility because the factors that are implicated in the development of problem behaviours do not necessarily maintain those behaviours (Cipani \& Schock, 2007). In other words, knowing who is more likely to self-injure does not provide the answer to why people self-injure. To understand why people engage in NSSI, it is essential to examine how these behaviours are reinforced and maintained over time by identifying the reasons or motivations people give for their self-injury, and the antecedents and consequences of these behaviours.

\section{SELF-REPORTED REASONS AND MOTIVATIONS FOR SELF-INJURY}

Reasons for NSSI can be divided into two broad categories: those that are motivated by intrapersonal factors and those that are motivated by interpersonal factors (Yates, 2004). Intrapersonal reasons are driven by a person's desire to alter their internal state, while interpersonal reasons are driven by a person's desire to communicate with others in their environment (Yates, 2004). In the following section, I review the most commonly reported intrapersonal and interpersonal reasons for self-injury, across a range of populations, before discussing the limitations of these studies. It should be noted that I have followed the precedent set by other researchers in using the words 'reason' and 'motivation' interchangeably to refer to the purpose of the self-injurious behaviour (Klonsky, 2007). 


\subsection{Intrapersonal Reasons}

The most common self-reported intrapersonal reasons for NSSI reflect a need to decrease, eliminate, or escape from unwanted negative emotions (e.g., anger), cognitions (e.g., suicidal ideation, traumatic memories), affect states (e.g., dissociation), and physiological conditions (e.g., tension). Additionally, many people report self-injuring to punish themselves which, in all likelihood, stems from the experience of negative emotions (Chapman et al., 2006) or cognitions as will be discussed later in this chapter.

Given my approach of inspecting individual items where possible, I have divided the reasons reported in the literature according to whether they fall under the categories of emotions/affect, cognitions, or physiological states. Of course, it should be noted that these intrapersonal events are all intricately interconnected and, as a result, they overlap experientially and conceptually. The complex interplay of experiences that results in named emotion is explicated by Barrett, Gendron, and Huang (2009) as follows:

\footnotetext{
The basic idea is that during emotional experience ("how do I feel?") and emotion perception ("is the rat afraid?"; "is my friend angry?"; " is my dog guilty?), representations of internal sensations from the body (experienced as affect) and external sensations from the world are made meaningful via the process of categorization (just as visual sensation are transformed into sight). This categorization uses emotion knowledge that has been learned via prior experience. Together, different recipes (the combination and weighting of these three sources of information-sensations from the world, sensations from the body, and prior experience) create the variety of mental states that represent your own feelings of your experience or someone else's behavior named with emotion words. (p. 431)
}

Although quantitative explorations of the reasons and motivations for NSSI cannot do justice to this complexity, they can provide insight into people's internal experiences and how these experiences contribute to self-injury.

\subsubsection{Emotional reasons}

Emotional motivations for self-injury predominate in measures designed to assess why people self-injure, and can be conceptualised along two intersecting continua of arousal (i.e., bodily activation) and valence (i.e., pleasantness) (Barrett, 1998). The conceptualisation of NSSI as a strategy for regulating aversive emotions 
or mood states has led researchers to primarily focus on the role of negatively, rather than positively, valenced emotions in the maintenance of self-injury.

\subsubsection{Specific emotions}

A number of specific emotions, such as anger, frustration, loneliness, and excitement have been investigated in studies of why people self-injure; these emotions have been differentially endorsed as reasons for NSSI across diverse populations of study participants. Reasons for self-injury that describe the elimination or expression of anger and frustration, both high arousal, negative emotions, have been reported by a majority of the participants in several studies (Brown et al., 2002; Laye-Gindhu \& Schonert-Reichl, 2005; Nixon et al., 2002). It is typically unclear whether the anger and frustration experienced by participants is directed at themselves or other people as studies seldom differentiate between selfand other-directed anger. One of the few studies that did make this distinction found that $63 \%$ of a community group of adolescents reported self-injuring because they were angry with themselves, whereas only 39\% reported self-injuring because they were angry with others (e.g., parents, friends) (Laye-Gindhu \& Schonert-Reichl, 2005). ${ }^{6}$

Low arousal, negative emotions that have been identified as reasons for NSSI in both community and psychiatric populations include feeling empty, lonely, and helpless (Favazza \& Conterio, 1989; Nixon et al., 2002; Oyefeso et al., 2008; Swannell, Martin, Scott, Gibbons, \& Gifford, 2008). Like high arousal, negative emotions, the particular low arousal, negative emotions measured, and the extent to which they are endorsed, depends on what questionnaire is used and the population sampled.

One emotion that has been examined in several NSSI studies is loneliness. In the first large-scale survey study to examine the motivations for self-injury, $47 \%$ of an all female sample reported injuring themselves "to feel less lonely" (Favazza \&

\footnotetext{
${ }^{6}$ To maintain consistency, all the percentages in this chapter have been reported without decimal places. Where percentages were reported with one or more decimal places in the original source, I have rounded these figures to zero decimal places.
} 
Conterio, 1989, p. 286). In contrast, 76\% of adolescent inpatients reported injuring themselves to "reduce a feeling of being utterly alone" (Swannell et al., 2008, p. 101) and $63 \%$ of community-based adolescents reported injuring themselves because they "felt all alone" (Laye-Ghindhu \& Schonert-Reichl, 2005, p. 452).

Few positive emotions have been investigated as reasons for NSSI and when they are included in questionnaires, the results tend to be variable. For example, selfinjuring for excitement was endorsed by $53 \%$ of the adolescent inpatients in one study (Swannell et al., 2008), but by only 7\% of the adolescent inpatients in another study (Nixon et al., 2002). This large discrepancy is surprising considering the items were worded very similarly and both study samples consisted of adolescent inpatients. The instructions on how to complete the questions may have differed between measures, but these instructions were not reported.

Concluding whether excitement motivates adolescents to self-injure is impossible from these studies. It is equally as difficult to determine whether excitement is a common motivation for NSSI in adult inpatient or community populations because other studies that have examined self-injuring for excitement did not report how many of their participants endorsed this reason (Kumar, Pepe, \& Steer, 2004; Osuch, Noll, \& Putnam, 1999). It is likely that self-injuring for excitement is motivated by boredom, which may be more common among adolescent and adult inpatients in restricted environments, but further research is necessary to determine whether this is the case.

\subsubsection{Specific affect states}

The two affect states that are most commonly investigated in studies about the reasons and motivations for self-injury are depression and dissociation. Over $80 \%$ of the community-based and inpatient adolescent participants in two separate studies endorsed self-injuring because they were depressed or trying to cope with depressed mood, making this the most commonly endorsed motivation for NSSI in these samples (Laye-Gindhu \& Schonert-Reichl, 2005; Nixon et al., 2002). A lower 
rate of endorsement (58\%) for the item, "to feel less depressed" (p. 286), was found among women in Favazza and Conterio's (1989) study.

In general, items about using NSSI to manage dissociation do not appear to rate as highly as depression items. Among two adult samples, 38\% to 57\% of people reported self-injuring to end dissociative states (Briere \& Gil, 1998, Favazza \& Conterio, 1989). Similar results were found in a group of hospitalised adolescents where just under half reported self-injuring "to stop feeling numb or out of touch with reality" (Nixon et al., 2002, p. 1337). In contrast, $87 \%$ of the inpatient adolescents in Swannell et al.'s (2008) study endorsed self-injuring to "decrease an empty feeling" (p. 101) making this the second most highly endorsed reason for NSSI in this sample.

Non-suicidal self-injury has not only been reported as an anti-dissociation strategy, but also has occasionally been examined as a means of inducing dissociation. A substantial proportion of adolescents (30\%-82\%) have reported injuring themselves to induce feelings of numbness, which provided some respite from their overwhelming emotions (Laye-Ghindhu \& Schonert-Reichl, 2005; Swannell et al., 2008). Apart from these two studies, self-injuring to induce, rather than reduce, dissociation does not appear to have been widely investigated.

\subsubsection{General emotional experiences}

Some studies included items that lacked specificity and referred to general, aversive emotional or affective experiences, such as "to stop bad feelings" (LloydRichardson et al., 2007, p. 1189), "to cope with emotional pain" (Oyefeso et al., 2008, p. 230), and "to get rid of intolerable emotions" (Klonsky, 2009, p. 263). Although these items were endorsed by a majority of the participants in these studies, it is impossible to isolate the specific emotions they refer to.

\subsubsection{Cognitive reasons}

\subsubsection{Specific cognitions}

Along with emotional reasons for NSSI, particular cognitions, especially to do with suicidal ideation or traumatic memories, have been identified by numerous 
people as motivations for their self-injurious behaviour. Self-injury, as a strategy to prevent suicidal ideation or attempts, has been reported by $41 \%$ to $74 \%$ of community-based and inpatient adolescents (Laye-Gindhu \& Schonert-Reichl, 2005; Nixon et al., 2002; Swannell et al., 2008). Certainly, endorsing self-injury as survival is consistent with the consumer perspectives presented in Chapter 1 (see pp. 15-16).

However, in studies where participants have been asked to rank their reasons for NSSI, averting suicidal thoughts has not been rated very highly. Only $7 \%$ of women with BPD rated "to prevent me from acting on suicidal feelings" as one of their top three reasons for NSSI (Shearer, 1994, p. 525), while 6\% of university students rated "to avoid the impulse to attempt suicide" as a primary reason for selfinjury and $18 \%$ rated it as a secondary reason (Klonsky, 2009, p. 264).

A number of people also report using self-injury as a way of distracting themselves from traumatic memories. In a clinical sample, in which over $90 \%$ of the participants had been sexually abused, 58\% reported self-injuring as a "distraction from memories" and 39\% to "stop flashbacks" (Briere \& Gil, 1998, p. 615). Using selfinjury to avoid or distract oneself from negative memories has also been reported by more than half of two inpatient adolescent samples (Nixon et al., 2002; Swannell et al., 2008).

A lower rate was found among a group of women diagnosed with BPD - only 15\% endorsed self-injuring to "keep bad memories away" (Shearer, 1994, p. 525)— but caution is required when interpreting these results as the women who participated in this study were asked to rank their top three reasons for NSSI. Consequently, the results may underestimate the number of women with BPD who self-injure to avoid or escape negative memories. Unfortunately, detailed information about the content of the memories or flashbacks is not reported in any of these studies.

\subsubsection{General cognitive states}

As with the general affect items, some researchers have included vague items about the cognitive motivations for NSSI in their questionnaires. For example, $72 \%$ 
of the women in Favazza and Conterio's (1989) sample reported self-injuring "to control their mind when it is racing" (p. 286), but the content of these thoughts is unclear. Likewise, 55\% of a group of secondary school students recalled self-injuring to get their mind off their problems and $20 \%$ to prevent themselves from "thinking bad thoughts" (Laye-Gindhu \& Schonert-Reichl, 2005, p. 452). Once again, these items are too general to provide sufficient information about the specific thoughts or problems that motivated these people to injure themselves.

\subsubsection{Physiological reasons}

\subsubsection{Releasing pressure or tension}

Items that refer to physiological reasons for self-injury often describe NSSI as a means through which to release tension. It should be noted that the release of tension is closely linked with self-injuring to cope with negative emotions. Indeed, it is probable that experiences of tension are triggered by intense emotions (Chapman et al., 2006). Furthermore, a number of questionnaire items actually specify the release of emotional tension. Eighty-five percent of a group of university students reported that their primary motivation for self-injuring was "to release emotional pressure that builds up inside me", while the remaining $15 \%$ reported it as their secondary motivation for engaging in NSSI (Klonsky, 2009, p. 263). In Briere and Gil's (1998) study, 77\% of the participants reportedly self-injured to "release pent-up feelings" (p. 615).

Measures used in other studies have not specified the release of emotional tension per se, but have rather referred to the elimination of tension in general. In two studies, one involving women diagnosed with BPD (Kleindienst et al., 2008) and one involving adult psychiatric inpatients (Herpertz, 1995), tension release was the most frequently reported motivation for NSSI. Similarly high rates of endorsement ( $45 \%-74 \%$ ) for the utility of NSSI as a tension release or relaxation strategy have been found in other studies (Favazza \& Conterio, 1989; Laye-Ghindhu \& Schonert-Reichl, 2005; Nixon et al., 2002). 


\subsubsection{Inducing stimulation}

Aside from using self-injury to release tension, people have also reported selfinjuring to produce stimulation, an experience which is often likened to a drug high (Kleindienst et al., 2008; Oyefeso et al., 2008; Swannell et al., 2008). For example, 47\% of a clinical sample of women with BPD endorsed using NSSI to "achieve a kick or high" (Kleindienst et al., 2008, p. 233) and 66\% of a clinical sample of adolescents endorsed using NSSI "to experience a high that feels like a drug high" (Swannell et al., 2008, p. 101). Inducing stimulation through self-injury can be thought of as comparable to self-injuring for excitement, which was discussed earlier.

Another motivation for self-injury that is rarely investigated and, when it is examined, seldom endorsed is using self-injury to induce sexual arousal. In a sample of women with BPD, only 5\% reported self-injuring "to provide a sense of physical release that feels much like sexual release"(Shearer, 1994, p. 525), while $12 \%$ of another clinical sample purportedly self-injured to experience "sexual arousal or pleasure" (Briere \& Gil, 1998, p. 615).

The role of pain as a stimulus has also been explicitly investigated in some of the measures of the reasons and motivations for self-injury. However, in these items, physical pain is presented as a means through which people can gain control over, or distract themselves from, unpleasant emotions or affect states. For example, 92\% of adolescent inpatients reported self-injuring because physical pain distracted them from their emotional pain (Swannell et al., 2008). Other items that have been endorsed by more than half of the participants in two separate studies include using self-injury to "produce a pain I can control" (Klonsky, 2009, p. 263) and "to feel something, even if it was pain" (Lloyd-Richardson et al., 2007, p. 1189). In all of these items, the primary motivation for NSSI appears to be the control or regulation of affect, which is made possible through the distraction provided by experiencing pain. 


\subsubsection{Self-punishment}

Self-punishment is often one of the most highly endorsed reasons for selfinjury. In three adult clinical samples, self-punishment received the highest level of endorsement of all the reasons provided for NSSI (Briere \& Gil, 1998; Brown et al., 2002; Shearer, 1994). However, it is a construct that defies simple classification within the emotional, cognitive, or physiological categories discussed thus far because it could feasibly fit within all three of these categories. Some studies have demonstrated that the desire to punish oneself through self-injury can be triggered by positive and negative experiences or affect states. For example, 50\% of hospitalised adolescents reported self-injuring to "punish self for being bad/bad thoughts" and 26\% reported self-injuring to "punish self for feeling good" (Nixon et al., 2002, p. 1337). In another study of adolescent inpatients, a majority endorsed the motivations to "punish myself for being bad" (84\%), to "punish myself for positive feelings/experiences" (68\%), and to "punish myself for telling secrets" (53\%) (Swannell et al., 2008, p. 101).

Self-punishment was also primarily driven by negative self-evaluations in a sample of women with BPD; 49\% endorsed using self-injury "to punish myself for being "bad" in some way (e.g., angry, selfish, etc.)" (Shearer, 1994, p. 525). In Oyefeso and colleagues' (2008) study, 68\% of the participants used NSSI to express their self-hatred, while Laye-Gindhu and Schonert-Reichl (2005) found that more than $60 \%$ of the adolescents in their study were motivated to self-injure because they did not like themselves and felt like failures.

It is hardly surprising that people report injuring themselves as punishment for both positive and negative experiences, given that NSSI is associated with low self-esteem (Brausch \& Gutierrez, 2010; Claes, Houben, et al., 2010). If the desire to self-punish is driven by low self-worth, it is plausible that people would blame themselves for any negative experiences they have and feel undeserving of positive experiences. In such cases, both negative and positive experiences may lead to feelings of guilt and shame which, in turn, could trigger self-injurious behaviour. It 
is thus clear from the examples discussed that self-injury can be used as a form of punishment in the context of negative emotions and cognitions about oneself, and for experiencing positive emotions.

\subsection{Interpersonal Reasons}

Interpersonal reasons for self-injury, which reflect support-seeking or avoidance, have not been investigated as thoroughly in the literature as intrapersonal reasons for self-injury, and when they have been included in measures assessing why people self-injure, they are seldom endorsed as highly as intrapersonal reasons. Concerns about perpetuating damaging stereotypes about self-injury as a purely attention-seeking behaviour may have encouraged researchers to avoid investigating intrapersonal reasons for NSSI (Nock, 2008). However, some people do claim to use self-injury to communicate with those around them or to exert control over their interpersonal environment (Nock, 2008; Yates, 2004) and, as a result, it is important to examine these motivations.

\subsubsection{Support-seeking}

As is common with all of the research investigating motivations for NSSI that I have presented thus far, the number of people who report using self-injury as a way of eliciting attention and support from others varies between studies. In one adult clinical sample, $40 \%$ of the participants endorsed self-injuring to "get attention, ask for help" but only $16 \%$ reported injuring themselves to get attention from their therapist and 9\% to receive medical attention (Briere \& Gil, 1998, p. 615). The discrepancy in these ratings may indicate that NSSI is used more frequently to elicit support and attention from family members or friends, rather than clinicians.

Indeed, over $50 \%$ of a sample of secondary school students were motivated to injure themselves to receive more attention from their friends or parents (LloydRichardson et al., 2007). Similarly, 41\% of community-based adolescents reported injuring themselves so that others would notice them (Laye-Gindhu \& SchonertReichl, 2005). Much lower rates of attention-seeking were reported in an adolescent inpatient population; only $10 \%$ recalled self-injuring to "get care or attention from 
others" (Nixon et al., 2002, p. 1337). Among a clinical group of women diagnosed with BPD, 17\% reported using self-injury as an indirect way of obtaining care and support from others because they felt unable to request this support verbally (Shearer, 1994).

The need for support and care often seems to occur in the context of distressing, overwhelming emotions. Over $70 \%$ of one group of adolescent inpatients reported injuring themselves to demonstrate to others how angry and hurt they felt (Swannell et al., 2008), whereas approximately one third of two different samples of secondary school students were motivated to hurt themselves to show others how desperate they were (Laye-Gindhu \& Schonert-Reichl, 2005; Lloyd-Richardson et al., 2007). Some adolescents have also reported using NSSI as a way to hurt, shock, irritate, or get back at people (Laye-Gindhu \& Schonert-Reichl, 2005; Swannell et al., 2008), or to feel more connected to a group of peers (Lloyd-Richardson et al., 2007).

\subsubsection{Avoidance}

Even less commonly investigated interpersonal reasons involve using NSSI to avoid having to complete tasks, fulfil responsibilities, or face the consequences of particular actions. More than $50 \%$ of a group of secondary school students reported self-injuring "to avoid school, work, or other activities" (Lloyd-Richardson et al., 2007, p. 1189). However, in another sample of secondary school students, only $16 \%$ endorsed self-injuring because they wanted to avoid certain tasks (Laye-Gindhu \& Schonert-Reichl, 2005). Further research is necessary to determine whether avoiding responsibilities is a common motivation for NSSI across different study populations.

\subsection{Limitations}

The literature on self-reported reasons and motivations for NSSI is limited in several ways. First, researchers investigating why people injure themselves on purpose typically develop questionnaires based on clinical experience and extant publications. As a result, only three self-report measures designed to assess why people self-injure have been used in more than one published study: the Functional Assessment of Self-mutilation (Lloyd-Richardson et al., 2007), the Self-injury 
Motivation Scale (Osuch et al., 1999), and the Inventory of Statements About Selfinjury (Klonsky \& Glenn, 2009).

The measures used in each study typically include various numbers of items about the same concepts (e.g., managing negative emotions through NSSI), but the different wording used in these items makes it difficult to reliably compare study results. Not only do some items include specific emotions, affect states, or cognitions, while others simply refer in general to negative feelings or thoughts, but the verbs used in each item differ. Some of the verbs contained in the items discussed include: to get rid of, to cope with, to express, to distract, to control, to release, to decrease, to feel, and to produce. These verbs hint at different functional mechanisms underlying the motivations for self-injury and, as such, items that include the same emotion or cognition word but have different verbs cannot be compared directly. For example, someone who endorses self-injuring to get rid of unpleasant feelings may not endorse self-injuring to express unpleasant feelings, as the latter implies a communicative function.

Furthermore, each of these questionnaires measures the reasons for NSSI in different ways. In some studies, participants rated their reasons for NSSI on likert scales (e.g., Kleindienst et al., 2008; Laye-Gindhu \& Schonert-Reichl, 2005); in others, they identified their primary and secondary motivations (Klonsky, 2009), ranked their top three motivations for self-injury (Shearer, 1994), or simply checked yes or no to identify which motivations were relevant to their experience of NSSI (Oyefeso et al., 2008). These diverse levels of measurement make it difficult to compare results across studies.

Second, the length of time since the participants' most recent episode of NSSI and their completion of the study measures varies widely both within and between studies. In Favazza and Conterio's (1989) study, 64\% of the participants had injured themselves in the month prior to the study whereas only $13 \%$ of the participants in Oyefeso's et al. (2008) study had self-injured in the previous 12 months. Moreover, many authors do not report how much time has elapsed between people's most 
recent self-injurious behaviour and their study participation, or even if that information was collected (e.g., Herpertz, 1995; Osuch et al., 1999; Swannell et al., 2008). One study did specify that participants had to have injured themselves within the previous two months to be included in the research (Brown et al., 2002), while another only included participants who had self-injured every month for the six months prior to the study (Nixon et al., 2002). Such specifications, however, are rare and the variation in time since last self-injury is a concern given that all of the studies reviewed thus far have relied on retrospective self-report and the accuracy of participants' memories are likely to decrease as the length of time since their last episode of NSSI increases (Tourangeau, 2000).

Finally, it is possible that the self-reported reasons for NSSI do not accurately reflect people's motivations for injuring themselves. Rather, people may be motivated to provide socially desirable responses (Nock, 2008) or their reasons may be post-hoc attributions to help them to make sense of their self-injury (Yates, 2004). The way in which people retrospectively appraise and justify their self-injurious behaviour, however, may be as clinically relevant as any factors that are truly motivating that behaviour: If people recall successfully escaping aversive affect states or accessing support through self-injuring, they are more likely to use NSSI in the future when they feel similarly distressed and overwhelmed (Chapman \& Dixon-Gordon, 2007; Kamphuis, Ruyling, \& Reijntjies, 2007).

\subsection{Summary}

In spite of the limitations associated with self-report studies of the reasons and motivations for self-injury, it is reasonable to conclude from this literature that self-injury is used primarily to alleviate or escape from aversive intrapersonal experiences, including emotions, cognitions, and physiological states (Klonsky, 2007; Nock, 2008). Although interpersonal reasons have been examined in fewer studies, where they have been included they are seldom as highly endorsed as intrapersonal reasons for NSSI. However, a substantial number of people still report using NSSI to 
communicate with, or exert influence over, others and, as such, interpersonal reasons for NSSI cannot be dismissed as irrelevant.

Rather, it is apparent that people injure themselves for multiple intrapersonal and interpersonal reasons. In studies where the actual number of reasons for NSSI has been reported, rates range from a median of four among drug dependent adults (Oyefeso et al., 2008) to an average of eight among adolescent inpatients (Nixon et al., 2002). However, these figures apply to participants' general experiences of selfinjury rather than to specific episodes. Only one study, to date, has examined the motives given by participants for a single episode of NSSI: Women with BPD identified an average of 10 reasons for their most recent self-injury (Brown et al., 2002). This study demonstrates that multiple reasons or motivations also underlie individual episodes of self-injury, at least amongst a sample of women with BPD. Further research is necessary to determine whether other populations, such as inpatient or community-based adolescents, would similarly report multiple motivations for single episodes of NSSI.

\section{SELF-REPORTED ANTECEDENTS AND CONSEQUENCES OF SELF-INJURY}

The literature on self-reported reasons for NSSI demonstrates that people are primarily motivated to hurt themselves to reduce, eliminate, or gain control over aversive intrapersonal experiences. In particular, there appears to be strongest support within these studies for reasons to do with negative emotions or affect states. Unfortunately, this research only suggests, but does not explicitly investigate, the emotional antecedents for NSSI, and fails to provide evidence that these emotions actually do change following acts of self-injury. Rather the process of change is typically implied in the way in which items are worded. For example, endorsing a reason, such as 'I self-injure to relieve negative emotions', assumes rather than directly asks whether negative emotions are present before and are eliminated after self-injuring. Several researchers therefore have retrospectively or prospectively examined specific emotional and physiological antecedents and 
consequences of self-injurious behaviour; cognitive antecedents and consequences have been largely neglected.

\subsection{Retrospective Studies}

Antecedents and consequences of NSSI are usually identified by asking research participants to indicate which affect or physiological states they commonly experience before and after NSSI. People routinely report decreased negative affect and increased positive affect following their engagement in self-injurious behaviour (Chapman \& Dixon-Gordon, 2007; Kamphuis et al., 2007; Kemperman, Russ, \& Shearin, 1997; Kleindienst et al., 2008). In one study, women recruited from a selfinjury support group completed a measure of negative and positive mood states, in relation to how they felt immediately before, after, and one day following episodes of NSSI (Kamphuis et al., 2007). Anger, depression, fatigue, and tension scores decreased significantly following self-injurious behaviour, while vigour (e.g., energetic, alert) scores showed a significant increase. Significantly more women reported a decrease in tension than any other internal state, leading the authors to conclude that NSSI functions primarily as a tension reduction strategy.

Similar results have been obtained in other studies. More than $90 \%$ of a sample of women diagnosed with BPD reported that the tension and pressure they felt prior to injuring themselves was significantly reduced after NSSI, while their ratings of positive affect items (e.g., "relaxed", "euphoria") were significantly higher after NSSI (Kleindienst et al., 2008). Analyses of change scores (i.e., rates of change from before to after NSSI) for university students showed that the high arousal, negative affect state-overwhelmed-decreased the most while low arousal, positive affect states - relief, calmness, satisfaction, and relaxation-increased the most (Klonsky, 2009).

However, not all people experience a decrease in negative emotion following NSSI. Almost half of a group of female inmates reported that the main emotional consequence of their most recent episode of NSSI was negative (e.g., sadness, guilt) (Chapman \& Dixon-Gordon, 2007). Other researchers have similarly found that self- 
conscious emotions, such as shame and guilt, are reported to increase following engagement in self-injurious behaviour (Kemperman et al., 1997; Laye-Gindhu \& Schonert-Reichl, 2005). It thus appears that while NSSI is an adaptive strategy to reduce overwhelming tension and certain aversive emotions, the act of self-injuring can also evoke other negative emotions (e.g., guilt). Given that the experience of aversive emotions precipitates NSSI, negative feelings consequent to self-injury have the potential to trigger future self-injurious behaviour, leading to a vicious cycle.

\subsection{Prospective Studies}

The application of prospective methodologies, such as ecological momentary assessment (EMA), to the study of NSSI is a recent empirical development. To date, only two studies have used EMA to examine the antecedents and consequences of NSSI. In one of these studies, women diagnosed with Bulimia Nervosa used palmtop computers to report their emotions, stressors, and any self-injurious behaviours or Bulimic symptoms at six, semi-random times a day for two weeks (Muehlenkamp et al., 2009). They were also asked to complete an additional set of questions each time they engaged in one or more of a set of pre-identified behaviours, including selfinjury. Analyses of the NSSI episodes showed a significant increase in negative affect and decrease in positive affect in the lead-up to the self-injurious behaviour. While positive affect increased significantly following NSSI, there was no significant reduction in negative affect.

The authors speculate that the lack of a significant decrease in negative affect may be an artefact of their small sample size and cannot be generalised to people without Bulimia who self-injure (Muehlenkamp et al., 2009). Given a larger sample, it may have been useful to examine the trajectories of particular affect states that precede and follow NSSI episodes. By analysing global positive and negative affect scores, significant decreases in specific negative emotions or affect states may have been overlooked. As previously discussed, evidence from retrospective self-report studies of the emotional antecedents and consequences of NSSI suggests that some 
negative emotions (e.g., anger) typically decrease following self-injury while other emotions (e.g., shame) may increase.

Ecological momentary assessment was also utilised by Nock and colleagues (2009) to investigate the antecedents and consequences of self-injurious thoughts and behaviours. Over two weeks, participants completed a set of questions twice a day and following any self-injurious thoughts or behaviours. Analyses showed that experiencing self- and other-directed anger, self-hatred, rejection, and numbness predicted NSSI episodes, while contrary to expectations, feeling sad or worthless decreased the likelihood of self-injury.

\subsection{Summary and limitations}

As was evident in studies of self-reported motivations for NSSI, one of the major limitations of the literature on the antecedents and consequences of self-injury is once again the failure of researchers to use standardised measures. Only two studies used a validated measure of emotion words (Kamphuis et al., 2007; Muehlenkamp et al., 2009); all others simply examined a range of emotions and affect states, presumably drawn from the NSSI literature. For example, Chapman and Dixon-Gordon (2007) report that they used a "standard list of nine emotions" (p. 546) but it is unclear where this list is derived from or why the emotions are considered standard. This use of disparate emotions and affect states makes it difficult to compare results across studies.

Although prospective investigations of the antecedents and consequences of NSSI involve real-time assessments of emotional states and are thus an improvement on retrospective studies, they nonetheless rely on participants being able to identify and accurately report on their emotional experience (Muehlenkamp et al., 2009) There is also the potential for such studies to have an iatrogenic effect in that people may be more likely to think about, and engage in, self-injury because they are participating in a daily study about NSSI. 


\section{LABORATORY STUDIES}

While phenomenological research of NSSI provides useful information about how the process of self-injury is perceived by those who engage in these behaviours, one of the drawbacks of most of these studies is their reliance on the single method of retrospective self-report. To address this limitation, some researchers have used self-injury proxies to try to simulate the psychophysiological experience of NSSI within a laboratory setting, while concurrently assessing self-reported affect states. While the external validity of such proxies is debatable (Klonsky, 2007), these studies nonetheless provide further evidence in support of the hypotheses that self-injury serves to reduce negative affect states and aversive psychophysiological arousal.

Both the cold pressor test (Russ et al., 1992) and guided imagery (Haines, Williams, Brain, \& Wilson, 1995; Welch, Linehan, Sylvers, Chittams, \& Rizvi, 2008) have been used to simulate NSSI within laboratory settings. In one study, participants completed the cold pressor test by immersing their left hands into a $10^{\circ} \mathrm{C}$ cold-water bath for four minutes, once a day, for three consecutive days. Analyses of averaged mood ratings before and after the cold pressor test showed that women who experienced no pain during NSSI reported significantly lower depression, anxiety, anger, and confusion scores, and significantly higher vigour scores after administration of the test (Russ et al., 1992). In contrast, women who experienced pain during NSSI did not report any significant differences in emotion. The only significant result for the control group was reduced anxiety.

These results lend support to the notion that NSSI functions as an affect regulation strategy although it is unclear why this was not the case for women who experienced pain during self-injury. It is possible that the cold pressor test was an inadequate self-injury proxy for these women, who may rely on reaching a certain threshold of pain during NSSI before they experience an alteration in mood (Russ et al., 1992).

Guided imagery has also been used in attempts to reproduce, within a controlled laboratory environment, the psychophysiological arousal patterns 
associated with NSSI, thus allowing researchers to examine whether NSSI serves a tension reduction function (Haines et al., 1995; Welch et al., 2008). In one study, individualised self-injury scripts were developed for men based on their descriptions of their most recent or salient episode of NSSI (Haines et al., 1995). Each script contained four discrete phases: setting the context, the lead-up to the event, the actual event, and the consequences of the event.

The men were instructed to visualise the events described in each script while their levels of psychophysiological arousal (e.g., heart rate, skin conductance response) were recorded. Psychophysiological arousal was found to increase in the lead-up to the recalled NSSI episode, decrease during the description of the actual self-injury, and remain low subsequent to the episode. This same pattern of arousal was not observed for scripts detailing neutral, aggressive, or accidental injury events. Self-reported ratings of negative emotions were also significantly lower in the final stage of the self-injury scripts when participants visualised the consequences of their self-injurious behaviour (Haines et al., 1995).

Welch and colleagues (2008) replicated the guided imagery procedure conducted by Haines et al. (1995) with a sample of people diagnosed with BPD. Similarly to Haines et al. (1995), participants' negative emotion and urge to selfinjure ratings, along with their skin conductance response rates, were significantly lower following the imagined NSSI episode, thus supporting the conceptualisation of NSSI as a negatively reinforced, emotion regulation strategy (Welch et al., 2008).

Laboratory-based studies lend support to the arguments that NSSI serves as both an emotion regulation and tension reduction strategy, but the external validity of self-injury proxies remain questionable (Klonsky, 2007). It is evident from the research reviewed thus far that NSSI is a complex, multi-determined behaviour (Suyemoto, 1998), making it very difficult to reliably recreate the experience of selfinjury and any accompanying contextual features within an experimental context. 


\section{CONCLUSION}

People endorse both intrapersonal and interpersonal motivations for NSSI, but it appears that intrapersonal reasons, particularly those pertaining to emotion regulation, are reported most frequently across a range of study populations. Indeed, conceptualising NSSI as an emotion regulation strategy is so pervasive that some authors even include this function within their definitions of the behaviour (e.g., Walsh, 2006). However, it is premature to define NSSI solely in terms of emotion regulation because of the limited research that has been conducted both on the role of cognitions in self-injurious behaviours and interpersonal reasons for NSSI.

Although cognitive precipitants for NSSI (e.g., suicidal ideation, traumatic memories) are not as highly or consistently endorsed as emotional ones, the impact of certain types of cognitions, such as negative automatic thoughts, has not been investigated. Of course as I discussed earlier, the experience of cognitions and emotions are intricately linked (Barrett et al., 2009). Indeed, it is probable that any cognitive motivations for self-injury are underpinned by the experience of aversive affect (Chapman et al., 2006). However, it is still necessary to analyse the extent to which different negative thoughts precipitate engagement in self-injury. Furthermore, it would be useful to determine whether particular thoughts evoke negative emotional responses that people with a history of self-injury find difficult to manage, or whether the experience of particular emotions triggers intrusive, negative thoughts. It is likely that a threshold is reached through the combination of sufficiently intense negative emotions, thoughts, and physiological arousal.

Interpersonal factors have been similarly neglected in studies of why people self-injure (Klonsky, 2007). As a result, caution is required before drawing definitive conclusions about the limited role of interpersonal factors in NSSI. Studies that do examine interpersonal reasons for self-injury need to take into account that people may not endorse these reasons for fear they will be perceived as manipulative or attention-seeking, although social desirability is unlikely to influence responses 
when questionnaires are completed anonymously (Klonsky, 2007; Nock, 2008). People also may lack insight into interpersonal motivations for their self-injurious behaviour or may not completely understand why they self-injure (Klonsky, 2007). It is also possible that intrapersonal reasons for NSSI are more salient and therefore easier to recall than interpersonal ones.

Furthermore, while the evidence to date suggests the most common interpersonal motivation for NSSI is communicating one's distress to others to receive attention and support, avoidance has seldom been investigated. However, future studies may find that a strict delineation between intrapersonal and interpersonal factors is not warranted. For instance, it has been suggested that certain interpersonal reasons for NSSI may indirectly serve an affect regulation function (Nock, 2008). People who are unable to effectively regulate their own emotions may look to others for help in managing their emotional well-being; NSSI is the strategy they use to communicate that such help is needed (Nock, 2008).

Extant research on why people are motivated to hurt themselves is also limited in that the majority of studies have relied on retrospective self-reports from convenience samples of predominantly Caucasian women. These results may not be generalisable to other populations. For example, studies involving secondary school and university students have demonstrated that some motivations for self-injuring differ according to gender (Klonsky \& Glenn, 2009; Laye-Ghindhu \& SchonertReichl, 2005; Lloyd-Richardson et al., 2007). In one study, males were more likely than females to report injuring themselves to provoke anger in others, whereas females were more likely than males to report injuring themselves as a form of selfpunishment (Lloyd-Richardson et al., 2007).

In the next chapter, I will discuss how the literature on the reasons for NSSI, and the antecedents and consequences of these behaviours, has been used to inform the development of both single- and multi-function models of self-injury. These models provide frameworks for organising the diverse reasons people give for self- 
injuring and have been instrumental in further developing our understanding of why people choose to hurt themselves on purpose. 


\section{CHAPTER THREE}

\section{The functions of self-injury: Theoretical conceptualisations}

\section{INTRODUCTION}

Successful treatment of self-injurious behaviours necessitates a sophisticated understanding of how these behaviours are reinforced and maintained over time. Various functional models of NSSI, each drawing on different psychological theories, have been proposed to account for why people injure themselves on purpose (Klonsky, 2007; Messer \& Fremouw, 2008; Suyemoto, 1998; Yates, 2004). Evidence for these models was originally derived from case studies and clinical experience (e.g., Menninger, 1935); more recently, the phenomenological and psychophysiological studies reviewed in Chapter 2 have been used to support the empirical and clinical validity of functional models of self-injurious behaviour.

In many cases, the development of what I shall call single-function models has simply been a matter of reframing people's self-reported motivations for selfinjury into specific NSSI models. Single-function models therefore can be conceptualised as being one step up from the reasons and motivations for NSSI that I reviewed in the previous chapter, in that they provide functional categories into which related reasons for self-injury can be grouped; in other words, the singlefunction models subsume individual reasons, motivations, antecedents, and consequences. For example, emotional reasons, antecedents, or consequences can be grouped within the overarching functional model of affect regulation; people who report self-injuring to release anger or manage feelings of depression are understood to self-injure to regulate negative affect.

Individual single-function models, however, cannot adequately account for why people are motivated to hurt themselves on purpose because NSSI, as an overdetermined behaviour, fulfils multiple functions at the same time (Klonsky \& Muehlenkamp, 2007; Suyemoto, 1998). To understand why any one person is 
engaging in NSSI, single-function models thus need to be considered concurrently. Without an overarching theoretical framework to connect these models, any attempt to integrate them is bound to be haphazard and, potentially, of limited clinical utility. Furthermore, I would argue that since little is known about the underlying mechanisms of these models, it is difficult to arrive at a parsimonious solution about which models should be grouped together.

Fortunately, several theory-driven, rather than data-driven, models have been proposed more recently, which I will refer to as multi-function models because they subsume individual single-function models. Some of these models have focused on specific populations, such as people with BPD (Crowell, Beauchaine, \& Linehan, 2009) or those with a history of trauma (Yates, 2004), or particular forms of selfinjury, such as cutting (Yip, 2005), and thus have limited applicability for my thesis because I am interested in how NSSI functions across different populations and multiple forms of self-injury.

Two multi-function models, however, that are applicable across populations and forms of NSSI are the Four Functions Model (FFM; Nock, 2008; Nock \& Prinstein, 2004, 2005) and the Experiential Avoidance Model (EAM; Chapman et al., 2006). These models have been proposed in an attempt to draw together the miscellaneous single-function theories of self-injury and, ultimately, to clarify the functions of NSSI. Unlike the disparate and often under-theorised single-function models, both the FFM and the EAM define function in accordance with social learning and behavioural perspectives (Chapman et al., 2006; Nock, 2008) and, as such, identifying the functions of self-injury requires simultaneously analysing the antecedents and consequences that cause or maintain self-injurious behaviours (Nock, 2008). In this way, functions of NSSI are conceptualised as temporal processes, rather than as categories of particular reasons or motivations. Defining the functions of self-injury in terms of the features which control the behaviour is thought to be fundamental to developing sophisticated treatment and prevention 
programmes that have the capacity to respond to individual characteristics (Nock, 2008; Nock \& Prinstein, 2004, 2005).

In this chapter, I briefly describe the basic premise of each of the singlefunction models, the evidence in support of the hypothesised functions, and the proposed causal mechanisms underlying these functions. I then examine the assumptions and empirical evidence in support of the EAM and FFM, before evaluating these two models within an epistemic values framework.

\section{SINGLE-FUNCTION MODELS OF SELF-INJURY}

According to recent reviews, the following eight single-function models have been repeatedly discussed within the literature on self-injurious behaviours: affect regulation, anti-dissociation, anti-suicide, self-punishment, sexual, interpersonal boundaries, interpersonal influence, and sensation-seeking (Klonsky, 2007; Messer \& Fremouw, 2008; Suyemoto, 1998). Although not an exhaustive list, I have chosen to concentrate my discussion on these single-function models because they have regularly featured in the NSSI literature as explanations for why people self-injure.

\subsection{Affect regulation}

The affect regulation model proposes that people self-injure to reduce or eliminate intense, negative emotions or affective arousal (Chapman et al., 2006; Klonsky, 2007). Evidence in support of this model is primarily derived from the selfreport studies of reasons for NSSI and the laboratory studies that I reviewed in Chapter 2. These studies have typically found that self-injury functions as an adaptive strategy to manage aversive affect and arousal, making affect regulation the most empirically supported, single-function model of NSSI (Klonsky, 2007).

Only one measure - the Inventory of Statements About Self-injury (ISAS) has been developed specifically to assess the single-function models of NSSI by grouping empirically supported reasons and motivations for NSSI into 13 functional subscales (Klonsky \& Glenn, 2009). ${ }^{7}$ Using the ISAS with a group of university

\footnotetext{
7 In developing the ISAS, Klonsky and Glenn (2009) aimed to include all the functions identified within the NSSI literature, as well as additional functions identified by researchers, clinicians, and
} 
students, Klonsky and Glenn (2009) found that affect regulation was the most highly endorsed function for self-injury.

Despite the compelling evidence from these studies that NSSI functions as an affect regulation strategy, there is limited understanding of how self-injuring actually enables people to regulate their emotional experiences. Both psychological and biological explanations have been proposed to account for this effect (Klonsky, 2007). Psychological explanations have focused on the sense of control that may be gained through expressing overwhelming emotions (Suyemoto, 1998) or engaging in self-care (Klonsky, 2007). People who struggle to cope with psychological distress may be more adept at attending to their physical wounds, thus providing them with a sense of mastery over their distress (Klonsky, 2007).

Biological explanations have focused on pain reduction and mood elevation following the release of endogenous opiods (see Sher \& Stanley, 2008 for a review), or the role of physical stimulation in triggering an attentional shift (Niedtfeld et al., 2010). In a brain imaging study, Niedtfeld and colleagues (2010) identified that thermal stimulation (warmth or painful heat), experienced in the context of hyperarousal elicited by negative images, was followed by reduced limbic activity in both controls and participants diagnosed with BPD. They hypothesise that sensory stimulation causes an attentional shift and although both groups demonstrated this shift, it may be that the intense affective arousal experienced by people with BPD when distressed necessitates that they use more painful methods of self-stimulation (e.g., cutting) in order to sufficiently shift their attention (Niedtfeld et al., 2010).

The hypothesis that self-injuring causes an attentional shift is consistent with reports from people that the physical sensation of NSSI distracts them from the emotional distress they are experiencing (Himber, 1994; Polk \& Liss, 2009). Additionally, it is possible that seeing blood prompts a shift of attention from aversive, internal experiences to the site of the physical injury (Glenn \& Klonsky, the ISAS. 
2010b), which may be particularly useful for people who do not feel pain during NSSI. Caring for the wounds might then serve to maintain this focus on the physical body, rather than on emotional distress.

Indeed, the sight of blood has been identified as an important component of the ritual of self-injury for some people as it is associated with calmness and tension relief (Glenn \& Klonsky, 2010b). Although the mechanism through which blood exerts this effect is unknown, it is possible that seeing blood either results in heart rate deceleration or heightened activation of the sympathetic nervous system, which is then followed by an intense parasympathetic reaction because there is no immediate danger (Glenn \& Klonsky, 2010b). Both of these physiological processes-heart rate deceleration and parasympathetic rebound-would result in the suppression of negative emotions (Glenn \& Klonsky, 2010b).

\subsection{Self-punishment}

Self-injury is thought to function as self-punishment by providing a means through which people can denigrate themselves or communicate how angry they are at themselves (Klonsky, 2007). Self-punishment was the second most highly endorsed function of NSSI, after affect regulation, in a population of university students (Klonsky \& Glenn, 2009) and, as I demonstrated in Chapter 2 (see pp. 52 53), self-punishment is one of the most commonly reported reasons for NSSI. More specifically, studies where people have explicitly reported self-injuring to punish themselves (e.g., Nixon et al., 2002; Shearer, 1994), to regulate self-directed anger (e.g., Laye-Gindhu \& Schonert-Reichl, 2005), or to express self-hatred (e.g., Nock et al., 2009; Oyefeso et al., 2008) can all be interpreted as support for the selfpunishment model.

Beyond this phenomenological research, the role of self-punishment in the development and maintenance of NSSI is not well understood. Given that selfinjuring to alleviate anger or guilt, or to express self-hatred is emotionally driven, it is possible that self-punishment is a sub-category of the affect regulation model (Chapman et al., 2006). This is seemingly consistent with Klonsky's (2009) finding 
that although affect regulation and self-punishment items were the most commonly endorsed motivations for NSSI among university students, affect-regulation motivations were typically reported as primary while self-punishment motivations were reported as secondary. Self-punishment therefore may be one specific type of affect regulation.

A number of specific psychological mechanisms have been proposed to underlie the self-punishment function of NSSI. Drawing on self-verification theory (Swann, Hixon, Stein-Seroussi, \& Gilbert, 1990) and cognitive dissonance theory (Festinger, 1978), Chapman et al. (2006) propose that when the disconfirmation of core self-beliefs or cognitive dissonance leads to aversive affect, people may selfinjure as a form of punishment. Getting the punishment they 'deserve' (i.e., selfinjury) validates their negative self-beliefs and restores cognitive balance, which in turn reduces aversive affect. In this way, "self-injury may be experienced as familiar, ego-syntonic, or self-soothing in the face of distress" (Klonsky \& Muehlenkamp, 2007, p. 1050).

Social learning theory (Bandura, 1977) also provides a possible explanation for why people punish themselves through self-injury (Chapman et al., 2006). Socially conditioned relationships between other-inflicted punishment and subsequent relief may generalise to self-punishment for any perceived or actual transgressions. People may also self-injure as a form of pre-emptive self-punishment to minimise or avert punishment by others.

If self-punishment can be subsumed within the affect regulation model, then the physiological mechanisms proposed to underlie affect regulation (e.g., attentional shift as a result of pain or the sight of blood) should be applicable to selfpunishment. Furthermore, self-injuring as a form of punishment can result in a sense of satisfaction (Nock, 2010), which may be related to the release of endorphins.

\subsection{Anti-dissociation}

Anti-dissociation, like self-punishment, has also been categorised as a type of affect regulation (Suyemoto, 1998). According to this model, self-injuring terminates 
depersonalisation or dissociation through the generation of feeling (Klonsky, 2007). As discussed in Chapter 2 (see pp. 47-48), empirical evidence in support of the antidissociation model has been mixed (Klonsky, 2007); 38\% to 87\% of adolescents and adults who self-injure report doing so to end dissociation or feelings of emptiness (Briere \& Gil, 1998, Favazza \& Conterio, 1989; Nixon et al., 2002; Swannell et al., 2008).

Once again, it is likely that self-injury fulfils an anti-dissociative function because the physical sensation and/or the sight of blood trigger certain physiological reactions (Klonsky \& Muehlenkamp, 2007; Polk \& Liss, 2009; Schoppmann, Schröck, Schnepp, \& Büscher, 2007). One such reaction may be the orienting response (Pavlov, 1927), which is the increased cortical activity that occurs in response to novel stimulation (Chapman et al., 2006). Although the role of the orienting response in NSSI is yet to be empirically examined, Chapman et al. (2006) propose that an orienting response to physical pain or the sight of blood may be sufficient to end dissociation, although the intensity of this response should decrease over time as people become habituated to experiencing pain or seeing blood when self-injuring.

Identifying the mechanisms through which NSSI functions as an antidissociation strategy becomes more complicated when taking into account evidence that self-injury not only ends dissociative episodes, but also has been reported to induce dissociation by providing an escape from overwhelming, internal experiences (Himber, 1994; Laye-Ghindhu \& Schonert-Reichl, 2005; Swannell et al., 2008). In one study, retrospective, self-reported levels of dissociation peaked during episodes of NSSI in a group of female inpatients diagnosed with BPD, regardless of whether or not they experienced pain while self-injuring (Kemperman et al., 1997). It appears that NSSI fulfils an antithetical function to anti-dissociation in such cases.

\subsection{Anti-suicide}

Drawing on psychoanalytic theory, NSSI is conceptualised within the antisuicide model as a compromise between the urges to live and die; in other words, self-injury is a form of temporary self-destruction that serves as a substitute for 
suicide (Firestone \& Seiden, 1990; Menninger, 1935; Suyemoto, 1998). This notion of using NSSI to avert suicidal impulses or to manage suicidal ideation, discussed in both Chapters 1 (see pp. 15-16) and 2 (see pp. 48-49), has been supported in a number of self-report studies (Klonsky, 2007).

It is probable that the act of physical injury distracts people from ruminating about taking their own life through the attentional shift process described above and/or improves their mood, at least temporarily, perhaps through the release of endorphins, so that suicide is no longer as salient as the best or only option for dealing with their distress. In this way, self-injuring to avert suicide could also be subsumed under the general framework of affect regulation in that the act of injury may decrease intense, negative feelings associated with suicidal urges or cognitions (Klonsky \& Muehlenkamp, 2007).

\subsection{Sexual}

The sexual model of self-injury is also informed by psychoanalytic theory and contends that people self-injure for sexual satisfaction, to exert control over their sexual development, or to avoid or punish themselves for sexual behaviours or feelings (Suyemoto, 1998). Although a historically prevalent explanation for why people self-injure, the sexual model has rarely been empirically investigated and, when it has been, it has received negligible support (Briere \& Gil, 1998; Kemperman et al., 1997; Shearer, 1994).

That this model holds little weight in contemporary understandings of the functions of NSSI is evident in the fact that the ISAS does not include a subscale addressing the purported sexual functions of self-injury (Klonsky \& Glenn, 2009) and self-injurious behaviours linked to sexual arousal were explicitly excluded from one study (Polk \& Liss, 2007), perhaps because including such motivations would have blurred the distinction between NSSI and sadomasochism. For the few people who do report being sexually gratified through self-injury (e.g., Briere \& Gil, 1998), it is likely that this is associated with the release of endorphins. 


\subsection{Sensation-seeking}

Self-injuring to produce exhilaration or excitement falls within the sensationseeking model of NSSI and in studies where such reasons for NSSI have been examined, they have typically been endorsed by a low percentage of participants (Klonsky, 2007). Once again, it is likely that any heightened arousal following NSSI, which is sometimes likened to a drug high (Swannell et al., 2008), results from the release of endorphins.

It is possible that sensation-seeking lies on the opposite side of the same coin to anti-dissociation, in that the desire to seek positive emotional arousal through selfinjury may stem from feelings of dissociation. If sensation-seeking was simply another descriptor for anti-dissociation, both functions should be similarly endorsed. However, this does not appear to be the case as anti-dissociation is more frequently reported than sensation-seeking (Klonsky \& Glenn, 2009).

\subsection{Interpersonal boundaries}

The interpersonal boundaries model of self-injury, which has rarely been empirically examined perhaps owing to its psychoanalytic roots, is based on objectrelations theory and proposes that people self-injure in order to distinguish themselves from others and to assert their autonomy (Klonsky, 2007). The action of marking the body may engender a sense of control because such marks physically delineate the boundaries between self, other, and the environment (Klonsky, 2007; Klonsky \& Muehlenkamp, 2007).

\subsection{Interpersonal influence}

Self-injuring to influence others tends to be perceived as the most contentious single-function model of NSSI in light of pejorative depictions of people who selfinjure as attention-seeking or manipulative (Nock, 2008). Although self-injuring to access support or care seems to be fairly common (Klonsky \& Muehlenkamp, 2007), interpersonal functions for NSSI are less frequently endorsed than intrapersonal functions (Klonsky \& Glenn, 2009; Nock \& Prinstein, 2004). However, it is possible that these functions are not reported as often because they are considered to be 
socially undesirable (Claes, Klonsky, Muehlenkamp, Kuppens, \& Vandereycken, 2010).

The interpersonal influence model draws on behavioural theory in that the person's self-injury is thought to signal to others that support is needed, and when support is provided, the self-injurious behaviour is reinforced (Klonsky, 2007). Arguably, using self-injury as support-seeking strategy could be conceptualised as affect regulation by proxy; people who struggle to regulate their own affective arousal and negative emotions may need the help of others to cope (Nock, 2008). Although the causal mechanism underlying this model is interpersonal reinforcement, people who self-injure to influence others may not be cognisant that their behaviour is being reinforced by others' responses, which would also influence how frequently they endorsed interpersonal functions (Klonsky, 2007).

\subsection{Summary and limitations}

A range of single-function models, which subsume the reasons, antecedents, and consequences of NSSI discussed in detail in Chapter 2, have been proposed to account for why people self-injure in the absence of suicidal intent. On the surface, some of these models appear to have little in common, but once they are considered in more depth, it becomes apparent that many of the models can be incorporated within an affect regulation framework and, in all likelihood, share the same or similar underlying mechanisms.

Furthermore, as an overdetermined behaviour (Suyemoto, 1998), self-injury is thought to serve multiple functions at the same time which makes it necessary to integrate the single-function models into a cohesive, clinically useful paradigm. One of the most promising ways to achieve this integration has been through the use of behavioural theory as an overarching framework. Behavioural models successfully capture the commonalities and differences of the diverse single-function models because they define the function of a behaviour by identifying both the antecedents and consequences of that behaviour (Nock \& Prinstein, 2004). The result is a paring down of the functions of NSSI into four, simple contingencies: intrapersonal and 
interpersonal consequences that are either negatively or positively reinforced (Nock, 2010).

\section{MULTI-FUNCTION MODELS OF SELF-INJURY}

The FFM (Nock, 2008; Nock \& Prinstein, 2004, 2005) and EAM (Chapman et al., 2006) are two promising behavioural models of NSSI that are garnering varying degrees of empirical support (e.g., Anderson, 2009; Armey \& Crowther, 2008;

Klonsky \& Glenn, 2009; Lloyd-Richardson et al., 2007). In the following section, I will examine the assumptions and research evidence in support of each of these models, before evaluating them according to well-established epistemic values.

\subsection{The Four Functions Model}

\subsubsection{Assumptions of the FFM}

The FFM (Nock, 2008; Nock \& Prinstein, 2004, 2005) proposes that self-injury can be categorised according to whether it serves an automatic (i.e., intrapersonal) or social (i.e., interpersonal) purpose, which, in turn, is either positively or negatively reinforced. These two dimensions intersect to form four primary functions of NSSI: automatic negative reinforcement (i.e., to eradicate or escape from negative thoughts or affective states), automatic positive reinforcement (i.e., to induce positive, physiological states), social negative reinforcement (i.e., to escape interpersonal events or demands), and social positive reinforcement (i.e., to get attention or desired objects) (Nock, 2008; Nock \& Prinstein, 2004, 2005). The term automatic in the context of the FFM refers to self-imposed reinforcement rather than actions that are carried out without conscious awareness (Nock \& Prinstein, 2004, 2005). In a later publication, Nock (2010) revised the FFM terminology to refer instead to automatic consequences as intrapersonal and social consequences as interpersonal. This is consistent with the language used by other NSSI researchers and, as such, the terms intrapersonal and interpersonal are used in this thesis.

In light of the limited empirical support for interpersonal functions, Nock (2008) presented an expanded version of the FFM, which includes hypotheses about the potential mechanisms underlying the social functions of NSSI (see Figure 1). The 
expanded model explains why people may use NSSI as a way to communicate with others. Drawing on theories of animal behaviour and anthropological studies, Nock (2008) suggests that self-injury may be used as a signal to convey strength and fitness or distress. People may begin to self-injure because less costly and less severe communication strategies have not produced desired results.

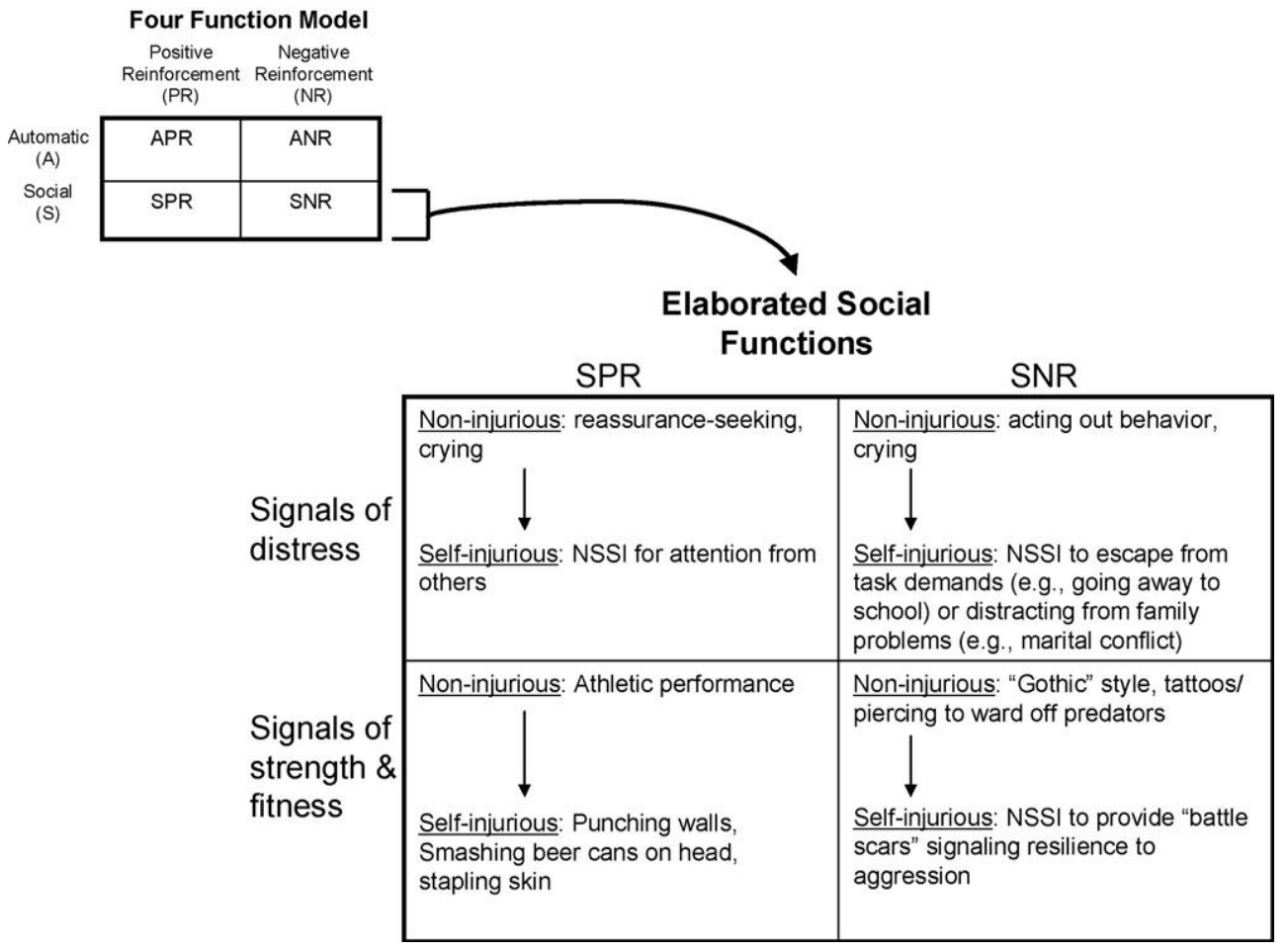

Figure 1. The elaborated FFM. Reproduced from "Actions speak louder than words: An elaborated theoretical model of the social functions of self-injury and other harmful behaviours," by M. Nock, 2008, Applied and Preventive Psychology, 12, p. 164.

For example, if a person seeks comfort from others and comfort is not provided, they may resort to NSSI as a way to gain attention and validation (i.e., self-injury is a signal of distress). If this strategy is successful, they may be more likely to use it in the future when similarly distressed. Likewise, someone may use self-injury to avoid or escape from fulfilling interpersonal obligations when less extreme behaviours (e.g., acting out) have failed. Alternatively, people may injure themselves to prevent victimisation by others or to demonstrate their connectedness with a particular social group (i.e., as a signal of strength or fitness). Once again, if 
self-injury proves to be an effective means of displaying one's strength or fitness to others, it is likely to be reinforced (Nock, 2008).

\subsubsection{Empirical support for the FFM}

To test the hypothesised four functions of self-injury, adolescent inpatients completed the Functional Assessment of Self-mutilation (FASM; Lloyd, Kelley, \& Hope, 1997, cited in Nock \& Prinstein, 2004), which includes a list of 22 reasons for engaging in self-injury (e.g., "to stop bad feelings", "to punish yourself").

Participants were able to endorse multiple reasons. The majority of the participants had injured themselves at least once in the past year, while almost half reported injuring themselves more than 10 times.

After categorising the FASM reasons for self-injury into the four hypothesised functional domains of negatively or positively reinforced intrapersonal and interpersonal consequences, Nock and Prinstein (2004) conducted a factor analysis which confirmed their model's goodness-of-fit with the data. Further analyses provided evidence that the four functions represented associated, but distinct paradigms.

Although this study supported the four function conceptualisation of NSSI, not all functions were equally endorsed (Nock \& Prinstein, 2004). Negatively reinforced, intrapersonal scores were significantly higher than the scores for the other three functions, while the scores for the positively reinforced, intrapersonal function were significantly higher than both the negatively and positively reinforced interpersonal functions. Additionally, $24 \%$ to $53 \%$ of the participants endorsed intrapersonal items, but only $6 \%$ to $24 \%$ of participants endorsed interpersonal items, suggesting that while adolescent inpatients self-injure for multiple reasons, the primary function of their self-injury is intrapersonal emotion regulation (Nock \& Prinstein, 2004).

Research with community-based adolescents provided further support for the structural validity of the FFM, although in contrast to Nock and Prinstein's (2004) findings, interpersonal functions received similar levels of endorsement in this 
sample as intrapersonal functions (Lloyd-Richardson et al., 2007). Furthermore, the endorsement of the different functions of NSSI varied according to the severity of the adolescents' self-injurious behaviour. Minor NSSI (i.e., hitting, pulling out hair, inserting objects, biting, or excoriation) was only significantly associated with intrapersonal functions, while moderate/severe NSSI (i.e., cutting, carving, burning, self-tattooing, scraping or erasing skin) was significantly associated with both intrapersonal and interpersonal functions. Adolescents within the moderate/severe category also used more types of NSSI and injured themselves more often than those in the minor NSSI category.

It thus appears that adolescents who have a more chronic history of NSSI are more likely to endorse multiple functions of self-injury. Furthermore, it is possible that people who are experiencing particular forms of psychopathology may be more likely to endorse certain functions of NSSI. Unfortunately, research efforts to identify clinical correlates of specific functions have yielded discrepant results. For example, past suicide attempts and current suicide ideation were significantly associated with all four functions among community-based adolescents (Lloyd-Richardson et al., 2007), whereas suicide attempts and hopelessness were only associated with intrapersonal, negative reinforcement among adolescent inpatients (Nock \& Prinstein, 2005).

Depressive symptoms have been associated with both intrapersonal (Hilt, Cha, \& Nolen-Hoeksema, 2008; Lloyd-Richardson et al., 2007) and interpersonal reinforcement (Nock \& Prinstein, 2005). Although it seems counter-intuitive that depressive symptoms and suicidal ideation would be significantly correlated with social functions, people may be using NSSI as a way to communicate to others that they are depressed and in need of support (i.e., social positive reinforcement) (Nock \& Prinstein, 2005), or to avoid obligations that they feel unable to fulfil because of their depressive symptoms.

In sum, there is evidence to support four behavioural functions of NSSI, but it appears that intrapersonal consequences, particularly those that are negatively 
reinforced, are more commonly endorsed than interpersonal functions. However, social desirability (Claes, Klonsky, et al., 2010) or a lack of awareness about how the behaviours are functioning may lead to the underreporting of interpersonal functions (Klonsky, 2007). Further research needs to be conducted to determine whether certain individual risk factors, diagnostic categories, or socio-cultural environments are more commonly associated with specific functions.

\subsection{The Experiential Avoidance Model}

\subsubsection{Assumptions of the EAM}

Non-suicidal self-injury is conceptualised within the EAM (Chapman et al., 2006) as a form of experiential avoidance, a functional diagnostic dimension defined as:

the phenomenon that occurs when a person is unwilling to remain in contact with particular private experiences (e.g., bodily sensations, emotions, thoughts, memories, behavioral predispositions) and takes steps to alter the form or frequency of these events and the contexts that occasion them. (Hayes et al., 1996, p. 1154)

Avoidance is thus broadly conceptualised by Hayes and colleagues (1996) to include all escape and avoidant behaviours, which serve to change intrapersonal events and the environmental factors that cause such events to occur.

Given that experiential avoidance is a functional diagnostic dimension, Hayes et al. (1996) argue that numerous mental disorders can be reframed as forms of experiential avoidance for particular client subgroups, thus facilitating the integration of diverse theoretical approaches and promoting research on specific behaviours across traditionally demarcated fields. Clinical syndromes such as Obsessive Compulsive Disorder, BPD, and Substance Use can be classified as experiential avoidance because they all involve clients experiencing aversive, intrapersonal events and then using unhelpful coping strategies in an attempt to avoid these experiences (Hayes et al., 1996).

In developing the EAM, Chapman and colleagues (2006) have followed Hayes et al.'s (1996) lead by reconceptualising self-injurious behaviour as a form of experiential avoidance. The EAM proposes that people experience an unwanted, 
negative emotional response (e.g., anger or frustration) to a particular stimulus (e.g., a fight with their partner) that they are unable to tolerate or manage. As a result, they feel an urge to escape from the undesirable state of arousal. To reduce or eliminate the distress that they are feeling, they injure themselves which provides them with a sense of relief. However, this relief is temporary, necessitating the continued use of self-injury to regulate their emotions and, as a result, the behaviour becomes an automatic way of responding to negative, emotional arousal (see Figure 2).

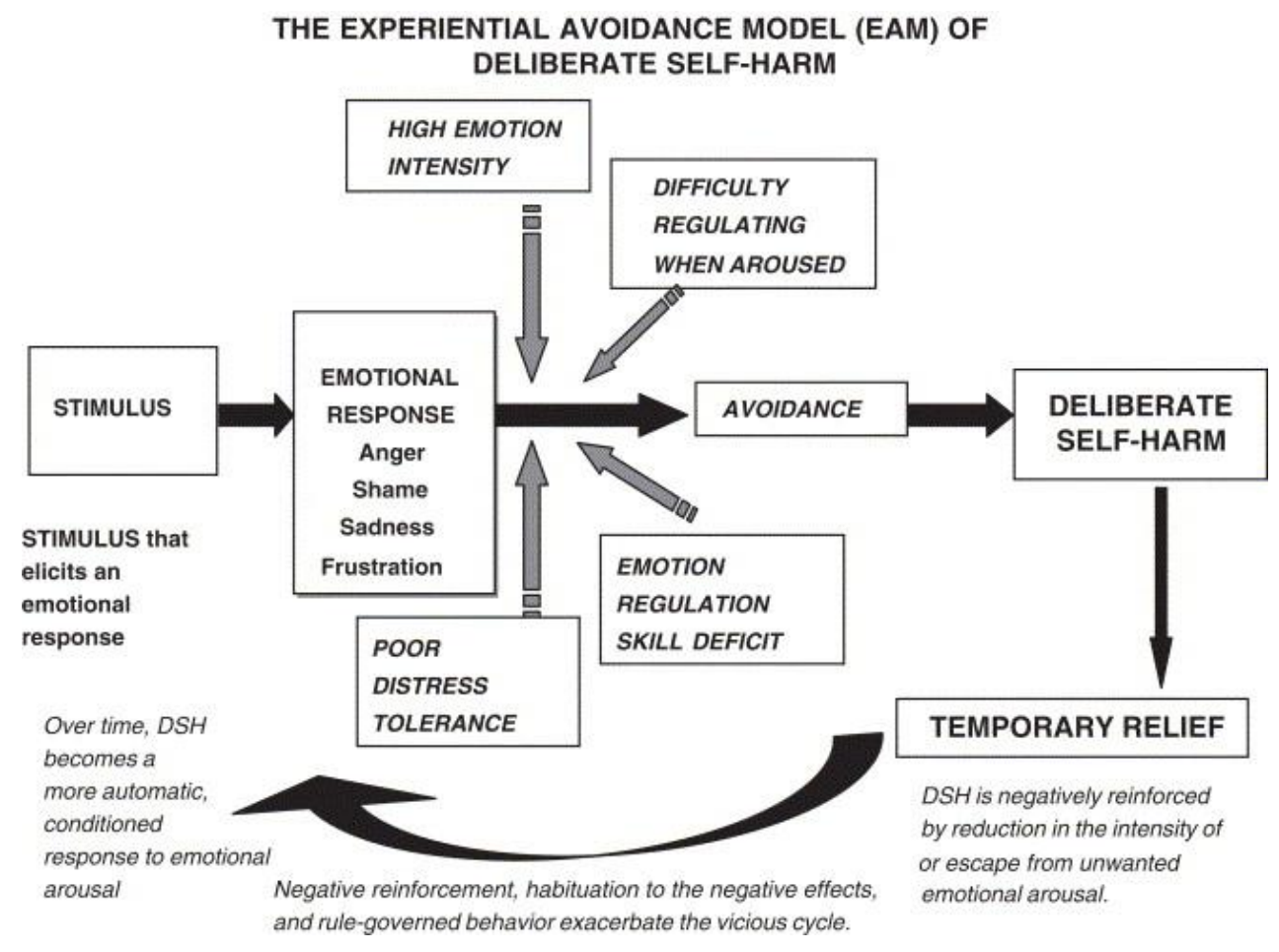

Figure 2. The Experiential Avoidance Model. Reproduced from "Solving the puzzle of deliberate self-harm: The experiential avoidance model," by A.L. Chapman et al., 2006, Behaviour Research and Therapy, 44, p. 373.

There are three key assumptions underlying the EAM. First, Chapman, and colleagues (2006) maintain that NSSI functions primarily as a behaviour of emotional avoidance although they do not discount the possibility that individuals may also utilise self-injury to escape from, or avoid, other unwanted internal experiences such as thoughts, memories, and bodily sensations. Second, NSSI is negatively reinforced 
because the reduction or elimination of unwanted internal states (particularly aversive emotions) is a direct consequence of the self-injurious behaviour. Third, the model suggests that experiential avoidance is a functional response class and, as a result, people who self-injure will also use other experientially avoidant strategies (e.g., thought suppression, substance use).

By drawing on diverse psychological literatures to explain (a) why individuals who self-injure may have heightened experiential avoidance response tendencies, (b) what mechanisms could underlie the function of experiential avoidance in people who self-injure and (c) why NSSI persists over time, Chapman et al. (2006) construct a comprehensive, functional model of self-injury. Although they briefly consider how innate temperamental factors, such as impulsivity, may contribute to heightened experiential avoidance response tendencies, they focus predominantly on the way in which people experience and regulate their emotions.

More specifically, the model proposes that if people who self-injure experience their emotions very intensely, they may find it difficult to control their heightened arousal levels, which in turn may increase their susceptibility to using NSSI as an experiential avoidance strategy. Alternatively, people who self-injure may not experience emotions more intensely, but rather may be unable to tolerate emotional distress. Low distress tolerance may be more extreme when experiencing emotions that typically induce a desire to escape or avoid (e.g., shame). The authors also suggest that emotion regulation skills could be a key factor in determining whether individuals are likely to utilise experientially avoidant behaviours. People who are unable to regulate their emotions effectively, either through the lack of skills or a failure to employ skills when highly aroused, may be at risk of attempting to avoid or escape aversive emotions through the use of NSSI (Chapman et al., 2006).

Although these hypotheses - that people may be susceptible to self-injury because they experience emotions more intensely than others, have low distress tolerance, and/or are unable to regulate their emotions effectively-are useful in identifying people at risk of engaging in NSSI, they do not account for why self- 
injury is a form of avoidance. Three underlying mechanisms are hypothesised to support the theory that NSSI functions primarily as behaviour of emotional avoidance: The act of self-injuring causes the release of endogenous opiates which provide a sense of analgesic relief; the physical pain of self-injury distracts from emotional pain; and/or self-injury is used as a form of punishment for perceived wrongdoing, or to avert actual punishment, and once sufficiently punished, emotional arousal decreases (Chapman et al., 2006). Of course, these are consistent with the proposed mechanisms for the single-function models discussed earlier in this chapter but, as I have already discussed, empirical evidence in support of these hypotheses is lacking (Chapman et al., 2006).

If NSSI does function as a form of avoidance, it becomes necessary to determine how the avoidance is maintained over time. According to the EAM, the repetitive nature of NSSI is potentially related to four factors (Chapman et al., 2006). First, it is more likely that aversive emotions will recur with an increased intensity and frequency if a person attempts to avoid or escape from these emotions by engaging in NSSI. Future attempts to regulate these emotions establish a cyclical pattern of repeated self-injury. Second, people who are unwilling to fully experience their aversive emotions may never learn that such emotions can be tolerated and, as a result, may come to see NSSI as the only or best solution for emotional regulation. Third, people who adopt a verbal rule equating NSSI with emotional release may never give themselves the opportunity to learn alternative ways of coping or may fail to learn from the negative effects of self-injury. Finally, people may simply habituate to the characteristics and negative consequences of NSSI over time, which makes it less likely that they will cease engaging in the behaviour.

\subsubsection{Empirical support for the EAM}

The three key assumptions of the EAM - that NSSI is primarily an emotionally avoidant behaviour, that it is negatively reinforced, and that people who self-injure will also engage in other avoidant behaviours - have been empirically investigated to varying degrees albeit not within Aotearoa New Zealand. 
Evidence that NSSI is an emotionally avoidant, negatively reinforced behaviour is primarily drawn from the self-report, laboratory, and ecological momentary assessment studies reviewed in Chapter 2 (see pp. 44-62). Additionally, research on the functions of NSSI discussed earlier in this chapter overwhelmingly supports the primacy of intrapersonal, negative reinforcement (Klonsky \& Glenn, 2009; Nock \& Prinstein, 2004).

The hypothesis that self-injury is a negatively reinforced, avoidant behaviour has also been examined by testing the relationship between the frequency of people's self-injurious behaviours and their intrapersonal experiences. Aversive selfawareness (i.e., the experience of negative thoughts and feelings about oneself) predicted engagement in NSSI among university students (Armey \& Crowther, 2008), providing further support for the experiential avoidance function of selfinjury. However, this study concentrated on antecedents that predicted NSSI, rather than on the consequences that may have reinforced and maintained the selfinjurious behaviours over time.

Studies that have examined whether the frequency of NSSI is related to negatively reinforced consequences have yielded mixed results. Decreases in negatively valenced, high arousal affect states and increases in positively valenced, low arousal affect states following NSSI, predicted lifetime cutting rates among university students, partially supporting the negative reinforcement hypothesis of NSSI (Klonsky, 2009). Among female inpatients with eating disorders, self-injury frequency was similarly related to increases in positive affect subsequent to NSSI, although this relationship did not reach statistical significance (Claes, Klonsky, et al., 2010). Decreases in negative affect and increases in positive affect following selfinjury suggest that NSSI may be both negatively and positively reinforced.

The final assumption of the EAM, that people who self-injure will engage in other avoidant behaviours (i.e., that NSSI fits within an avoidant response class), has received some support. Undergraduate students with a history of NSSI, who were matched to controls on psychological distress levels, reported significantly higher 
rates of avoidant coping (Andover, Pepper, \& Gibb, 2007). Another study of young adults similarly found significantly higher levels of avoidant coping among people with a history of self-injury when compared to those who had never self-injured (Hasking, Momeni, Swannell, \& Chia, 2008). Moreover, in the latter study, as the severity of self-injurious behaviours increased, so did avoidant coping, which suggests that self-injury may belong in a response class of avoidant coping strategies.

However, it is not clear whether these avoidant coping strategies or behaviours (e.g., thought suppression, substance use) mediate, moderate, or simply co-occur with NSSI. Recently, Najmi, Wegner, and Nock (2007) proposed that people who self-injure and are highly reactive to emotions will try to suppress their negative thoughts as a way of gaining control over their distress. However, unless thought suppression is used to focus on one particular distracter, it typically increases the frequency of the thoughts (Najmi at al., 2007) because "the intention to suppress a thought instigates a monitoring process that ironically increases the cognitive accessibility of the unwanted thought" (Wegner \& Zanakos, 1994, p. 616). Following this paradoxical increase in aversive thoughts and emotions, Najmi and colleagues hypothesised that people will graduate to self-injury because it serves as a focused distracter.

They found that the association between emotional reactivity and rates of selfinjurious behaviour was partially mediated by thought suppression, which suggests that in some incidents, self-injury may be utilised following an unsuccessful attempt to suppress distressing thoughts (Najmi et al., 2007). However, these cross-sectional results would need to be replicated longitudinally before reaching any definitive conclusions about the temporal sequencing of thought suppression and NSSI. Further research is needed to identify the exact nature of the relationships between NSSI and other forms of avoidance.

Finally, NSSI is associated with a number of clinical syndromes, such as Bulimia and Substance Use Disorders (Klonsky \& Muehlenkamp, 2007), that are 
similarly thought to function as forms of experiential avoidance (Hayes et al., 1996; Heatherton \& Baumeister, 1991). This lends support to the hypothesis that NSSI belongs in a functional response class of behaviours primarily aimed at escaping or avoiding negative internal states.

\subsection{Evaluating the FFM and the EAM}

Both the EAM and the FFM rely on operant conditioning principles to explain why people are motivated to injure themselves, and how this behaviour is reinforced and maintained over time. While the FFM includes intrapersonal and interpersonal functions which are either positively or negatively reinforced, the EAM focuses exclusively on the negatively reinforced, intrapersonal function of experiential avoidance. Epistemic values provide an evaluative framework, which can be used to identify the strengths and limitations of each of these models. Although I utilise this framework for my critique, it should be noted that epistemic values are not objective, definitive criteria, but rather subjective, multi-faceted constructs (Rooney, 1992). Nonetheless, they provide a useful starting point for comparing and contrasting theoretical models. Drawing on the work of Kuhn (1977) and McMullin (1983), Howard (1985) identifies and defines six epistemic values: predictive accuracy, internal coherence, external consistency, unifying power, fertility, and simplicity; values which I use to compare the EAM with the FFM.

\subsubsection{Predictive accuracy}

Arguably, the key criterion in determining the validity of a theoretical model is whether that model accurately predicts its hypothesised outcomes (Howard, 1985). To determine whether the EAM and the FFM have adequate predictive accuracy, it is first necessary to revisit the basic assumptions of each model. The FFM proposes that NSSI will serve four primary functions: intrapersonal negative and positive reinforcement, and interpersonal negative and positive reinforcement. In contrast, the EAM predicts that NSSI will serve one primary, intrapersonal function of experiential avoidance. Additionally, the EAM posits that individuals who self- 
injure to avoid or escape unwanted internal experiences will also use other experientially avoidant coping strategies (e.g., binge eating).

Both the EAM and FFM propose, in line with the principles of operant conditioning, that understanding the function of a behaviour involves identifying the antecedents and consequences of that behaviour. Furthermore, a consequence of self-injury would only be considered to reinforce self-injurious behaviour if it increased the likelihood of the person engaging in subsequent episodes of self-injury (Cipani \& Schock, 2007). However, establishing a direct, temporal relationship between consequences and future episodes of self-injury is extremely difficult. Someone may experience a particular consequence (e.g., increased attention from loved ones) following an episode of self-injury, but this does not mean that they injured themselves to elicit that consequence or that they will injure themselves to elicit the same consequence in the future (Nock, 2008).

Although the evidence to date supports the contention that there are four functions of NSSI, people routinely endorse intrapersonal, negative reinforcement as primary to the maintenance of their self-injurious behaviour. The EAM thus surpasses the FFM in terms of predictive accuracy as it is far more likely that a person will report engaging in NSSI to escape or avoid negative intrapersonal experiences, than any of the other three hypothesised functions of NSSI.

However, the predictive utility of the EAM and the FFM ultimately needs to be determined through prospectively and longitudinally assessing the antecedents and consequences of self-injurious behaviours; a challenging task in light of the necessary ethical constraints that accompany any NSSI research (Prinstein, 2008). It is likely that ecological momentary assessment and longitudinal survey methods will prove to be essential in testing and supporting the reinforcement hypotheses of the FFM and EAM (see Muehlenkamp et al., 2009; Nock et al., 2009).

\subsubsection{Internal coherence}

An internally coherent, theoretical model needs to be logically consistent; that is, all the elements of the model should fit together (Howard, 1985). The EAM 
provides a comprehensive overview of how the cycle of self-injury is triggered, reinforced, and maintained over time. As such, it reflects its premise that understanding the function of a behaviour requires identifying both the antecedents and consequences of that behaviour. The only potentially incongruent aspect of the EAM is that the experience of relief following NSSI is hypothesised to negatively reinforce self-injury. If relief is viewed as a positive affect state, then experiencing relief after self-injury would positively reinforce the behaviour (Klonsky, 2009). However, if relief is defined as an absence of distress (Watson \& Tellegen, 1985), then conceptualising it as a negatively reinforced consequence of NSSI is no longer anomalous (Klonsky, 2009).

In contrast to the EAM, the FFM lacks internal coherence because the conceptualisation of the model is inconsistent with the definition of function proposed by Nock and Prinstein (2004). In their paper, they define function as the antecedents and consequences that cause and maintain a behaviour, but they fail to include any antecedents in their model, which focuses exclusively on how selfinjurious behaviour is reinforced. The researchers acknowledge this shortcoming and attempt to address it in a subsequent paper (Nock \& Prinstein, 2005), yet instead of investigating specific antecedents, they focus on clinical correlates (e.g., suicide attempts) and contextual features (e.g., experience of pain while self-injuring). The cross-sectional nature of their data prohibits them from demonstrating whether these correlates and contextual factors actually trigger episodes of self-injury.

Furthermore, although Nock and Prinstein (2004) hypothesised four primary functions of NSSI, they found significantly more support for the intrapersonal negative reinforcement function over the other three functions. This begs the question whether a four functions model, where each of the functions is viewed as primary to the cause and maintenance of self-injury, is conceptually valid. It may be more prudent to consider the automatic negative reinforcement function as primary, with the other functions as secondary. It should be noted, however, that the intrapersonal and interpersonal functions of NSSI were similarly endorsed in one of 
the only other confirmatory factor analyses of the FFM (Lloyd-Richardson et al., 2007).

\subsubsection{External consistency}

Theoretical models that are consistent with established theories from the same or similar disciplines meet the criterion of external consistency (Howard, 1985). As previously discussed, well-established behavioural principles of operant conditioning are fundamental to both the EAM and the FFM. The multi-function structure of the FFM is also consistent with the extensive evidence base on stereotypical self-injury in developmentally disabled populations (Nock \& Prinstein, 2004 , 2005). Furthermore, in his elaboration of the social functions of the FFM, Nock (2008) draws on evidence from both anthropology and theories of animal behaviour (see section 3.1.1).

Through arguing that NSSI functions as an experientially avoidant behaviour, Chapman et al. (2006) rely on the burgeoning evidence base that suggests experiential avoidance is the key to understanding a range of psychological disorders. This literature forms the theoretical foundation of the EAM and was discussed in detail earlier in this chapter (see section 3.2.1).

\subsubsection{Unifying power}

Unifying power refers to a theoretical model's capacity to draw together diverse strands of knowledge into a cohesive whole (Howard, 1985). Both the FFM and EAM draw various single-function models of self-injury into more comprehensive models of NSSI. The FFM is an exhaustive multi-function model in that every single-function model presented in the literature could feasibly be categorised according to whether the purpose for self-injuring reflects an attempt to alter one's intrapersonal or interpersonal environment, and whether it is positively or negatively reinforced.

In contrast, Chapman and colleagues (2006) argue that many of the singlefunction models of NSSI propose that self-injury enables people to avoid, or escape from, unwanted internal experiences, although the emphasis on what the person 
who self-injures is trying to escape from or avoid varies between models. Given this commonality, they propose that the overarching construct of experiential avoidance subsumes the hypotheses put forward by many single-function models. Both the FFM and the EAM thus have the capacity to account for the multiple reasons, reviewed in Chapter 2, which people give for self-injuring.

\subsubsection{Fertility}

A model is considered fertile if it generates new directions for future research on the topic (Howard, 1985). Beyond proposing that NSSI serves four functions, Nock and Prinstein (2004) do not provide any hypotheses about why NSSI may serve different functions for different people, how these functions relate to one another, and what mechanisms underlie each of the four functions. While Nock (2008) did elaborate on the FFM in a subsequent paper, he focussed exclusively on the interpersonal functions of NSSI and, as a result, the intrapersonal functions remain under-theorised.

In comparison, the EAM proposes a gamut of testable hypotheses about why people who self-injure may have heightened experiential avoidance response tendencies, what mechanisms underlie the function of experiential avoidance in people who self-injure, and why NSSI becomes a habitual behaviour (see section 3.2.1).

\subsubsection{Simplicity}

The EAM meets the criteria of simplicity in its depiction of self-injury as a negatively reinforced, cyclical process carried out to avoid or escape from aversive internal states. It could be considered a limitation that the EAM to fails include positive reinforcement contingencies and does not account for any interpersonal functions of NSSI, given the evidence that people do use self-injury to communicate with, and influence, others (e.g., Lloyd-Richardson et al., 2007; Nock \& Prinstein, 2004). However, Chapman et al. (2006) acknowledge that NSSI likely serves multiple functions but are explicit in their assertion that the primary function of NSSI is emotional (experiential) avoidance. 
In comparison, the FFM has a broader scope with its emphasis on four primary functions of NSSI. The four functions of the FFM complement each other to cover a range of contingencies and on the surface the FFM appears to be a simple, falsifiable model. But the difficulty arises when, as discussed earlier, one attempts to determine how the functions relate to one another, whether the model proposes that people who self-injure are likely to endorse all four functions within and/or between episodes, or whether the functions served by self-injury will depend on contextual features and/or psychopathological symptoms. The manner in which the FFM is conceptualised provides no hypotheses for these questions and, as such, it could be thought of as an under-theorised model that is complicated by its over-inclusivity.

\section{THE CURRENT THESIS}

Although several single-function models have been proposed for why people self-injure, research has shown that NSSI serves multiple functions and, as such, individual single-function models are unable to account for the full range of NSSI functions. In contrast, multi-function models, particularly those conceptualised within a behavioural paradigm such as the FFM and the EAM, succeed at integrating diverse single-function models to present more comprehensive perspectives on selfinjurious behaviour.

Both the FFM and the EAM are empirically supported when compared on the basis of adherence to common epistemic values (Howard, 1985), but the EAM is a more internally coherent, fertile, and parsimonious theoretical model of why people hurt themselves on purpose. Additionally, the evidence to date suggests that people primarily injure themselves to regulate their emotional experiences and to punish themselves (Klonsky, 2007), which is consistent with the emphasis placed by Chapman and colleagues (2006) on emotional avoidance. Indeed, as discussed earlier in this chapter, all of the most empirically supported single-function models (e.g., affect regulation, self-punishment, anti-suicide, and anti-dissociation) can be unified under the rubric of experiential avoidance. 
In light of this evidence, my overarching research question for this thesis is: Does NSSI function primarily as an experientially avoidant behaviour within Aotearoa New Zealand? This is an important topic to investigate because this question has not been asked of populations living in Aotearoa New Zealand. As such, our current understanding of why people in this country self-injure is mostly limited to anecdotal reports and evidence gleaned from international studies, which have focused primarily on testing single- rather than multi-function models. This focus has resulted in particular forms of experiential avoidance, such as affect regulation, being extensively investigated, while other forms of experiential avoidance, such as cognitive avoidance, have been largely neglected. Given that developing a sophisticated understanding of the functions of NSSI is essential to effectively treat these behaviours, it is imperative that we do not simply assume that the functions of self-injury within Aotearoa New Zealand are comparable to those endorsed overseas, but rather that we investigate why people living here self-injure in order to establish a New Zealand-specific evidence base about NSSI.

That being said, my general hypothesis is that NSSI will primarily function as a way for people living in Aotearoa New Zealand to escape from or avoid aversive emotional experiences. Therefore, I did not expect to find that the functions of my participants' self-injurious behaviours would differ significantly from those endorsed by people living in other developed Western countries. If the EAM is a valid, explanatory framework for NSSI in Aotearoa New Zealand, this may have important clinical implications. In particular, it may warrant the investigation of Acceptance and Commitment Therapy (ACT) as a treatment for self-injurious behaviours because the central goal of ACT is to decrease experiential avoidance by enhancing psychological flexibility (Hayes, Luoma, Bond, Masuda, \& Lillis, 2006).

To determine whether NSSI functions primarily as an experientially avoidant behaviour within Aotearoa New Zealand, I designed and conducted three studies. For my first study, I interviewed people who had self-injured without suicidal intent in the previous 12 months to elucidate the temporal process of their most recent 
episode of self-injury. By asking about the situational, emotional, and cognitive antecedents and consequences of their self-injurious behaviour, I was able to functionally assess each episode of NSSI to determine whether the majority of these episodes fulfilled an experientially avoidant function as predicted by the EAM (Chapman et al., 2006).

In my second study, an online survey of people across Aotearoa New Zealand who had self-injured in the past 12 months, I examined three primary hypotheses, all of which were informed by the EAM (Chapman et al., 2006), extant literature on the functions of NSSI (e.g., Klonsky, 2007), and the results from my first study. First, I hypothesised that participants would endorse affect regulation as the primary function of NSSI both in relation to their most recent and general episodes of NSSI. Second, I expected participants to endorse intrapersonal functions of NSSI more strongly than interpersonal functions, both in regards to their most recent and general episodes of NSSI. Third, I hypothesised that participants would report a decrease in negative affect/emotion and an increase in positive affect/emotion following their most recent episode of self-injury. Although any increases in positive affect would oppose the exclusive focus on negative reinforcement in the EAM, the findings from my first study and other empirical evidence to date suggested that this possibility should not be excluded.

My final study involved comparing university students, with a history of NSSI, to those who had never self-injured, in order to determine whether people who have self-injured experience higher levels of negative emotions and/or thoughts, and use more avoidant coping strategies in general. Additionally, I was interested in investigating whether the experience of negative emotions and/or thoughts predicts the frequency of NSSI, and, if this is the case, whether these relationships are mediated by people's propensity to avoid aversive intrapersonal experiences. 


\title{
CHAPTER FOUR
}

\section{An Interpretative Functional Analysis of self-injury}

\begin{abstract}
"Behavior analysis is based on a pragmatic philosophy: what is true is what works. Behavior is understood in terms of its function, not its form, and function is always understood in relation to a context." (Hayes \& Bissett, 2000, p. 239)
\end{abstract}

\section{INTRODUCTION}

Most studies examining why people hurt themselves on purpose have relied exclusively on self-report methodologies, but few of these have qualitatively examined people's descriptions of their motivations for self-injury. As highlighted in Chapters 2 and 3, phenomenological research on NSSI has predominantly involved participants completing survey measures, many of which have been developed from the extant literature specifically for each study. People are typically presented with a predetermined set of reasons or motivations for NSSI and asked to endorse the items that reflect their experiences of self-injury.

Although the uniformity and brevity of surveys are an advantagequantitative methods are used to examine the functions of NSSI later in this thesis the information that can be extracted from such measures is limited in scope and depth. Quantitative and qualitative methodologies complement one another, and both are needed to clarify the functions of self-injury; quantitative research provides explanations of phenomena while qualitative research promotes understanding of those phenomena (Hjelmeland \& Knizek, 2010). Indeed, one of the research recommendations in the self-harm guidelines developed by the National Institute for Health and Clinical Excellence (2004) is to utilise qualitative designs to investigate why people hurt themselves on purpose.

Despite being limited in number, qualitative studies on NSSI to date have addressed diverse topics, including understanding the phenomenological experiences of men who self-injure (Russell, Moss, \& Miller, 2010), the role of parents in triggering adolescent self-injury (Kam-shing, Mei-yuk, \& Lam, 2003), and the 
"hostile care" received by women who seek help for self-injury from Accident and Emergency Departments (Harris, 2000, p. 167). However, elucidating the functions of self-injury has seldom been the focus of this research. Rather, the reasons or motivations for NSSI presented in these studies have emerged from discussions or written accounts of self-injurious behaviours in general.

These reasons, however, have been largely consistent with those reported in quantitative studies; for example, self-injury has been described as a way to regulate affect, self-punish, express emotions, and gain control (Harris, 2000; Kam-shing et al., 2003; Rissanen, Kylmä, \& Laukkanen, 2008; Russell et al., 2010). Unfortunately, none of these studies - qualitative or quantitative - about why people self-injure have been carried out in Aotearoa New Zealand. Furthermore, to my knowledge, in the majority of research on self-injurious behaviours within populations from Aotearoa New Zealand, these behaviours have been operationalised as DSH not NSSI (see for example Fortune, 2006; Garisch \& Wilson, 2010; Hatcher, Sharon, \& Collins, 2009; Skegg et al., 2003). Non-suicidal self-injury among New Zealanders thus remains a largely unexplored phenomenon. ${ }^{8}$

The striking absence of both qualitative and quantitative research on NSSI in Aotearoa New Zealand informed the methodology for my first study; I decided that it was important to ground my empirical work for this thesis in stories of self-injury told by people who have experiential knowledge of these behaviours. More specifically, I was interested in clarifying the functions of NSSI through learning about the temporal process of discrete self-injurious episodes. Gathering information about single episodes of NSSI to functionally analyse the behaviour is a technique that is used in DBT (Linehan, 1993a), but I have not found any research studies that have employed this method of analysis with typically developing populations who self-injure.

\footnotetext{
8 There are a few notable exceptions. For examples, see Curtis (2003) and Garisch (2010).
} 
In DBT, therapists complete chain analyses with clients to elucidate the specific events that lead to problem behaviours (including self-injury), along with the consequences that reinforce and maintain those behaviours (Linehan, 1993a; Lynch, Chapman, Rosenthal, Kuo, \& Linehan, 2006). A chain analysis is a precise, highly detailed form of behaviour analysis whereby individuals identify the momentary changes that occurred in a particular behaviour chain (Linehan, 1993b; Lynch et al., 2006). Identifying the behavioural trajectory of a single episode of selfinjury is necessary for two reasons. First, to formulate hypotheses about the functions of a problem behaviour, one needs to focus on specific episodes as particular behaviours, such as cutting, can serve multiple functions for the same person in different contexts (Cone, 1997). Second, the functions fulfilled by a particular behaviour may change over time (Cipani \& Schock, 2007; Dougher \& Hayes, 2000). For example, an adolescent may initially self-injure in a group context to access peer approval, but continue to self-injure privately to escape overwhelming, negative emotions.

In the current study, I concentrated on single episodes of self-injury because I wanted to elicit rich, detailed narratives about the process of self-injurious behaviours, rather than a description of the antecedents and consequences of people's amalgamated experiences of NSSI. To achieve this, I interviewed people about their most recent episode of NSSI, focusing specifically on what led up to these episodes and the consequences that followed. Identifying the antecedents and consequences of NSSI enabled me to analyse whether people's descriptions of the temporal process of self-injury were congruent with the trajectory depicted in the EAM and international studies, and to hypothesise about how many of these episodes fulfilled an experientially avoidant function.

\section{STUDY OVERVIEW}

Ethics approval for the current study was granted by the Multi-region Ethics Committee, a New Zealand Health and Disability Ethics Committee administered by the Ministry of Health. Gaining ethics approval involved extensive consultation with 
individuals and teams within the mental health services at the Capital and Coast District Health Board (CCDHB) and the Hutt Valley District Health Board (HVDHB), as well as with the Student Counselling Service at Victoria University.

Despite the inevitable delays that occurred during this consultation, the process was immensely beneficial in that it enabled me to refine and develop my research method in response to the questions and concerns posed by experienced consumer advocates and clinicians. Additionally, it prompted me to consider thoroughly the impact that my research may have on participants who identified as Māori given that consultation with Māori was a critical component of this ethical review process.

The current study was conducted in four stages. First, people who expressed interest in participating in the study were screened using the DSHI (Gratz, 2001), a self-report questionnaire designed to assess the form, frequency, and severity of nonsuicidal self-injurious behaviours. Second, if people indicated that they had injured themselves on purpose in the previous 12 months, they were invited to participate in an interview. Third, following each interview, I emailed or telephoned the participants to ask whether there was anything else they had thought of that they wanted to share. Finally, I sent each participant a questionnaire asking for feedback on their experiences of taking part in the study. To preserve the structure and flow of the original research process, I have chosen to present the method and results for each stage separately, before discussing whether the findings of this study are consistent with the EAM (Chapman et al., 2006) and international research on the functions of NSSI.

\section{STAGE ONE: SCREENING FOR SELF-INJURIOUS BEHAVIOURS}

\subsection{Method}

\subsubsection{Recruitment strategy}

In an attempt to interview a diverse group of people for this study, I recruited participants through mental health services and from the community. Recruiting participants from the community was vital to ensure that people who had not actively sought help for NSSI, who had not been referred to mental health services, 
or who were no longer engaged with such services were given the opportunity to participate. It also became apparent when consulting with consumer advocates and clinicians that restricting my recruitment efforts to mental health services may limit participation to people that clinicians considered 'well enough' to take part. One consumer advocate suggested that by granting clinicians power as the gatekeepers of study participation, I was privileging their authority over the autonomy of mental health consumers. Furthermore, recruiting participants from the community ensured that I was not relying solely on clinicians remembering to pass study information on to their clients.

To obtain ethics approval to recruit participants from the local mental health services, I had to submit locality assessments for each service that participated in the study. This involved first meeting with the heads of the CCDHB mental health service, the HVDHB mental health service, and the Student Counselling Service, and then consulting with consumer representatives, Psychology Advisors, and mental health clinicians from teams within these services. I was also required to consult with Māori representatives or organisations within each service to ensure that my study was culturally appropriate. Following email contact with team leaders and my attendance at team meetings, participant information sheets (see Appendix A) were given to a range of mental health teams.

The following CCDHB teams agreed to hand out my information sheets to clients who met the study inclusion criteria and might be interested in taking part: Youth Speciality Service, Community Alcohol and Drug Services, Regional Rangatahi Adolescent Inpatient Services, General Adult Mental Health Services, Team for Assertive Community Treatment, and Pember House Community Mental Health Team. Within the HVDHB, the adult community mental health teams, the Youth Speciality Service, and the Central Region Eating Disorders Service all agreed to distribute information sheets. Additionally, clinicians from the Student Counselling Service at Victoria University of Wellington agreed to distribute information sheets on my behalf. The information sheet invited people to contact me 
if they wanted more information about the research or if they wanted to participate in the study.

To recruit community-based participants, I advertised the study in four local community newspapers and placed posters about the study in relevant community venues (e.g., Evolve, which is a youth health service; the offices of the Wellington Mental Health Consumers Union; the Wellington People's Centre) and at various locations around Victoria University's Kelburn campus. The information about my study was also disseminated through a local youth mental health consumer network and to the members of a community public health organisation.

\subsubsection{Participants and procedure}

Adolescents and young adults between the ages of 16 to 34 years who had engaged in at least one type of non-suicidal, self-injurious behaviour within the past 12 months were invited to participate. This age range was selected because research has shown that deliberate self-harm (i.e., non-suicidal self-injury and suicide attempts) is most prevalent among females between the ages of 15 to 24 years and males between the ages of 25 to 34 years of age (Schmidtke et al., 1996). The lower age limit of 16 was selected because within Aotearoa New Zealand, people need to be at least 16 years of age to participate in research without parental consent. Additionally, only people who could speak English fluently were invited to participate. Other exclusion criteria listed on the information sheet were Intellectual Disability, engaging in NSSI exclusively during episodes of mania or psychosis, or current evidence of mania or psychosis. Although these criteria were not formally assessed, it was clear during the interviews that none of the participants met these criteria.

Potential participants were provided with a copy of the information sheet, in which they were assured of their right to withdraw from the study at any time without having to explain their reason(s) for withdrawal. Of the 34 people who contacted me to enquire about the study, one male and one female did not meet the inclusion criteria because they were older than 34 years of age. The 32 people (28 
female, 4 male) who consented via email or telephone to complete the screening survey were posted a copy of the survey, which included basic demographic questions and the DSHI (Gratz, 2001) (see Appendix B), along with a list of support organisations (see Appendix C) they could contact if they found answering the questions distressing. This list contained the contact details of six different support organisations including LifeLine, Youthline, and Warmline (i.e., a helpline run by people who have experienced mental illness). A total of 31 people completed the survey; one female self-excluded after reading the questions because she engaged primarily in indirect forms of self-injury such as disordered eating, rather than direct forms such as cutting. As a result, she decided that her experiences were not relevant to the current study. Each person received a movie voucher for completing the survey. ${ }^{9}$

\subsubsection{The Deliberate Self-harm Inventory}

As mentioned in the study overview, the DSHI (Gratz, 2001) is designed to measure the form, frequency, and severity of people's engagement in non-suicidal self-injurious behaviours. It was chosen for this study because the content best reflected the definition of self-injury used in this thesis (see p. 26). The DSHI contains 17 index questions about different types of NSSI (e.g., "Have you ever intentionally (i.e., on purpose) cut your wrist, arms, or other area(s) of your body (without intending to kill yourself)?") and if people endorse a behaviour, they are asked to respond to a further five questions about the age of onset, frequency, recency, duration, and severity of that particular type of NSSI. The final index question gives people the opportunity to list any other self-injurious behaviours that they have engaged in and then answer further questions about those behaviours.

Responses on the DSHI can be used to derive a continuous variable by adding the frequencies of NSSI types to give a total score or a dichotomous variable by categorising participants on the basis of whether or not they endorse having self-

\footnotetext{
${ }_{9}$ One participant received a $\$ 10$ voucher for the Warehouse instead of a movie voucher because she had hearing difficulties.
} 
injured (Gratz, 2001). Although the validity and reliability of the DSHI has not been extensively assessed, psychometric analyses to date have found adequate construct validity, good internal consistency $(\alpha=.79-.82)$, and high test-retest reliability for the rates of NSSI reported across two administrations $(r=.91-.92)$ (Fliege et al., 2006; Gratz, 2001; Gratz et al., 2011). For the purposes of this study, I modified question 10 of the DSHI (i.e., "Have you ever intentionally (i.e., on purpose) used bleach, comet, or oven cleaner to scrub your skin?") to exclude the word "comet" because it is an American product that is not sold in Aotearoa New Zealand.

\subsection{Results}

\subsubsection{Demographic and diagnostic information}

Twenty-seven $(87.1 \%)$ of the 31 people who completed the DSHI were female. Participants ranged in age from 16 to 33 years $(M=21.65, S D=3.99)$ and $27(87.1 \%)$ identified as Pākehā/New Zealand European. The remaining four participants identified as New Zealand/Samoan $(N=1)$, Indian $(N=1)$, Asian $(N=1)$, and Chinese $(N=1)$. Twenty-five $(80.6 \%)$ participants reported that they had received at least one mental health diagnosis; the mean age of first diagnosis was 17.48 years $(N=23, \mathrm{SD}=4.14)$. The modal number of reported diagnoses per participant was one (range: 0-5). Twenty-one (67.7\%) people reported having been diagnosed with depression, nine (29.0\%) with an anxiety disorder, four $(12.9 \%)$ with an eating disorder, three $(9.7 \%)$ with a personality disorder, two (6.5\%) with Bipolar Disorder, one with Schizophrenia (3.2\%), and three (9.7\%) with other diagnoses (e.g., Gender Dysphoria, Cyclothymia with psychosis). ${ }^{10}$

\subsubsection{Prevalence of different types of self-injury}

Interpreting the responses on the DSHI (Gratz, 2001) proved challenging, as many people seemed unable to accurately recall the information required to answer the questions. As a result, 30 participants responded to some or all of the frequency

\footnotetext{
${ }^{10}$ Although some participants had experienced psychotic episodes in the past, they were still included in the study as the majority of their NSSI, including their most recent episodes, had not occurred in the context of psychosis.
} 
questions (e.g., "How many times have you done this?") with estimates (e.g., "100150?", "unsure-3-5", "approx. 10") or written explanations (e.g., "countless", "too many times to remember", "don't know"). Similar responses were obtained for some of the other questions, such as those requiring people to identify when they last engaged in a particular form of self-injury (e.g., "sometime this year", "no idea, months back") and the number of years that they had used a particular form of selfinjury (e.g., "1-2 years (occasionally)”, "on and off but not a regular occurrence”). Given the difficulties of analysing this data, I emailed Kim Gratz to ask whether she had received similar responses and, if so, how she quantified these. She replied that since modifying the DSHI, she seldom receives qualitative answers (personal communication, August 22, 2008). The revised version of the DSHI instructs people to "Please write an actual number (e.g., 1, 5, or 15 NOT some, many, or few)" when reporting the number of times they have engaged in a form of NSSI and to "Please write the actual number of years you engaged in this behavior" when reporting the number of years that they have self-injured in a particular way.

If I had used the revised questionnaire ${ }^{11}$, I may have received fewer written explanations but, in all likelihood, the frequencies reported by participants would still have been estimates. Unless people had kept a detailed record of their selfinjurious behaviours, it is unlikely they would have been able to quantify how many times they had injured themselves in a particular way, or when these injuries occurred. The following comments, written at the end of two separate DSHI's, illustrate these difficulties: "Some of the "last time you did this" are guestimated due to not keeping any record of these events. Sorry." and "These are all approximate! To the best of my recollection." During one of the interviews, one participant who was reflecting on the process of filling in the DSHI commented that being asked to remember how many times you have self-injured was analogous to being asked how

\footnotetext{
${ }^{11}$ I did not use the revised questionnaire because I was unaware that it had been developed.
} 
many times you have worn high-heeled shoes; an impossible task considering you do not make a note of every time you wear a particular type of shoe.

An additional concern raised by asking people to recall the exact number of times they had injured themselves was highlighted by another person during an interview who said she had considered counting her scars to determine how many times she had injured herself. However, she had realised that this would still be inaccurate as some of the injuries had not left scars or the scars had faded over time. Knowing that people may feel compelled to count their scars for the purposes of a research study appalled me and, as a result, I modified the wording of the DSHI for my subsequent two studies.

However, I still had to somehow make sense of the DSHI data I had obtained for this study. I was primarily interested in extracting the following information from the DSHI: the different types of NSSI people had used, the number of times they had engaged in each type of behaviour, and the recency of those behaviours. As is evident in Table 2, I followed the precedent set by other researchers (e.g., Lundh, Karim, \& Quilisch, 2007; Whitlock et al., 2006) in choosing to categorise both the frequency and recency data.

Cutting was the most commonly endorsed type of NSSI with $96.8 \%$ of the participants reporting that they had cut themselves in their lifetimes, followed by severe scratching (83.9\%), and preventing wounds from healing (80.6\%). Use of at least one other type of NSSI was reported by 12 (38.7\%) participants, including grazing one's skin by rubbing a key back and forth, pouring boiling water over one's skin, and self-flagellation. On average, people reported having engaged in 8.03 types of NSSI in their lifetimes (SD $=2.69$, range: $3-13$ ).

As noted earlier, the most difficult information to analyse from the DSHI was the frequencies of NSSI because of people's tendency to report estimates or provide 
Table 2

Frequencies and recency of different types of NSSI

\begin{tabular}{|c|c|c|c|c|c|c|c|c|c|c|c|}
\hline \multirow{2}{*}{ NSSI type } & \multirow{2}{*}{$\begin{array}{c}N(\%) \\
\text { reporting } \\
\text { NSSI type }\end{array}$} & \multicolumn{5}{|c|}{$N(\%)$ reporting frequency of engagement in NSSI types } & \multicolumn{5}{|c|}{$N(\%)$ reporting recency of NSSI types } \\
\hline & & Never & 1 time & $\begin{array}{c}2-10 \\
\text { times }\end{array}$ & $\begin{array}{l}11-50 \\
\text { times }\end{array}$ & $>50$ times & $\begin{array}{l}\text { Within past } \\
\text { week }\end{array}$ & $\begin{array}{l}\text { Within past } \\
\text { month }\end{array}$ & $\begin{array}{l}\text { Within past } \\
6 \text { months }\end{array}$ & $\begin{array}{l}\text { Within past } \\
12 \text { months }\end{array}$ & $\begin{array}{l}>12 \text { months } \\
\text { ago }\end{array}$ \\
\hline $\begin{array}{l}\text { Cutting wrists, arms, or other } \\
\text { areas of body }\end{array}$ & $30(96.8)$ & $1(3.2)$ & $0(0.0)$ & $8(25.8)$ & $4(12.9)$ & $7(22.6)$ & $6(19.4)$ & $7(22.6)$ & $8(25.8)$ & $4(12.9)$ & $5(16.1)$ \\
\hline $\begin{array}{l}\text { Severe scratching to extent of } \\
\text { bleeding/scarring }\end{array}$ & $26(83.9)$ & $5(16.1)$ & $2(6.5)$ & $15(48.4)$ & $1(3.2)$ & $1(3.2)$ & $2(6.5)$ & $4(12.9)$ & $1(3.2)$ & $6(19.4)$ & $11(35.5)$ \\
\hline Preventing wounds from healing & $25(80.6)$ & $6(19.4)$ & $2(6.5)$ & $5(16.1)$ & $2(6.5)$ & $2(6.5)$ & $4(12.9)$ & $4(12.9)$ & $4(12.9)$ & $4(12.9)$ & $7(22.6)$ \\
\hline Sticking sharp objects into skin & $22(71.0)$ & $9(29.0)$ & $1(3.2)$ & $4(12.9)$ & $7(22.6)$ & $0(0.0)$ & $0(0.0)$ & $4(12.9)$ & $5(16.1)$ & $6(19.4)$ & $6(19.4)$ \\
\hline Carving words into skin & $19(61.3)$ & $12(38.7)$ & $2(6.5)$ & $13(41.9)$ & $2(6.5)$ & $0(0.0)$ & $0(0.0)$ & $2(6.5)$ & $1(3.2)$ & $3(9.7)$ & $13(41.9)$ \\
\hline $\begin{array}{l}\text { Carving pictures/designs/marks } \\
\text { into skin }\end{array}$ & $18(58.1)$ & $13(41.9)$ & $4(12.9)$ & $11(35.5)$ & $1(3.2)$ & $0(0.0)$ & $1(3.2)$ & $2(6.5)$ & $3(9.7)$ & $1(3.2)$ & $11(35.5)$ \\
\hline Burning with lighter/match & $18(58.1)$ & $13(41.9)$ & $1(3.2)$ & $8(25.8)$ & $5(16.1)$ & $0(0.0)$ & $1(3.2)$ & $0(0.0)$ & $3(9.7)$ & $4(12.9)$ & $9(29.0)$ \\
\hline Punching to extent of bruising & $17(54.8)$ & $14(45.2)$ & $1(3.2)$ & $10(32.3)$ & $1(3.2)$ & $0(0.0)$ & $1(3.2)$ & $1(3.2)$ & $6(19.4)$ & $1(3.2)$ & $8(25.8)$ \\
\hline Banging head to extent of bruising & $15(48.4)$ & $16(51.6)$ & $0(0.0)$ & $5(16.1)$ & $4(12.9)$ & $0(0.0)$ & $2(6.5)$ & $1(3.2)$ & $3(9.7)$ & $2(6.5)$ & $7(22.6)$ \\
\hline Burning with cigarette & $13(41.9)$ & $18(58.1)$ & $1(3.2)$ & $5(16.1)$ & $4(12.9)$ & $0(0.0)$ & $0(0.0)$ & $1(3.2)$ & $0(0.0)$ & $3(9.7)$ & $8(25.8)$ \\
\hline Biting to extent of breaking skin & $13(41.9)$ & $18(58.1)$ & $6(19.4)$ & $4(12.9)$ & $1(3.2)$ & $0(0.0)$ & $1(3.2)$ & $0(0.0)$ & $1(3.2)$ & $4(12.9)$ & $6(19.4)$ \\
\hline Rubbing glass into skin & $7(22.6)$ & $24(77.4)$ & $3(9.7)$ & $1(3.2)$ & $1(3.2)$ & $0(0.0)$ & $0(0.0)$ & $0(0.0)$ & $2(6.5)$ & $1(3.2)$ & $4(12.9)$ \\
\hline Rubbing sandpaper on body & $5(16.1)$ & $26(83.9)$ & $4(12.9)$ & $1(3.2)$ & $0(0.0)$ & $0(0.0)$ & $0(0.0)$ & $0(0.0)$ & $1(3.2)$ & $1(3.2)$ & $3(9.7)$ \\
\hline Dripping acid onto skin & $2(6.5)$ & $29(93.5)$ & $1(3.2)$ & $1(3.2)$ & $0(0.0)$ & $0(0.0)$ & $0(0.0)$ & $0(0.0)$ & $0(0.0)$ & $1(3.2)$ & $1(3.2)$ \\
\hline $\begin{array}{l}\text { Using bleach/oven cleaner to scrub } \\
\text { skin }\end{array}$ & $2(6.5)$ & $0(0.0)$ & $0(0.0)$ & $2(6.5)$ & $0(0.0)$ & $0(0.0)$ & $0(0.0)$ & $0(0.0)$ & $0(0.0)$ & $0(0.0)$ & $2(6.5)$ \\
\hline Breaking own bones & $1(3.2)$ & $30(96.8)$ & $1(3.2)$ & $0(0.0)$ & $0(0.0)$ & $0(0.0)$ & $0(0.0)$ & $0(0.0)$ & $0(0.0)$ & $0(0.0)$ & $1(3.2)$ \\
\hline
\end{tabular}

Note. $N$ reporting NSSI type may not equal frequencies as some participants endorsed type of NSSI, but did not report frequency.

Frequencies may not add up to $100 \%$ because of missing data. 
written explanations. When I calculated NSSI frequencies, I only included participants who had provided numerical data. ${ }^{12}$ For example, 30 people reported having cut themselves but only 19 provided numerical responses when asked how many times they had cut themselves.

\section{STAGE TWO: THE INTERVIEWS}

\subsection{Participants and procedure}

All 31 people who completed the DSHI (Gratz, 2001) were invited to take part in one or more interviews. Each person was sent a copy of the interview questions (see Appendix D), as advised by a Clinical Psychologist, because of the sensitive nature of the topic. Receiving a copy of the questions enabled potential participants to make a fully informed decision as to whether they would like to take part. The questions were prefaced by the following paragraph:

Please read the following questions carefully. The purpose of this study is to understand how you experience self-harm ${ }^{13}$ from your perspective, while at the same time addressing the research questions listed below. As a result, Robyn cannot guarantee that she will ask you all of these questions or that these will be the only questions she will ask you during the interview(s). The questions do, however, provide you with an idea about what kinds of topics will be discussed during the interview. Please consider carefully if you would be comfortable answering these questions before you decide whether you would like to participate in an interview.

Additionally, reviewing the questions beforehand gave participants who consented to be interviewed the opportunity to prepare themselves for the interview process. The benefits to participants afforded by this procedure were considered to outweigh the drawbacks that may have occurred from participants over-preparing answers (e.g., to make them more socially desirable).

The interview schedule was developed for this study and was informed by the research literature on the behavioural functions of self-injury. As discussed

\footnotetext{
${ }^{12}$ Whenever participants estimated NSSI frequencies, I chose to include the most conservative value. For example, if a person reported that they had cut themselves 150-200 times, I took 150 as the frequency of cutting. If someone wrote thousands of times, I used 1,000 as the frequency.

${ }^{13}$ In my initial study, I referred to non-suicidal self-injurious behaviours as self-harm because this is the term that is most commonly used in Aotearoa New Zealand to describe these behaviours. However, in light of the terminological discrepancies between self-harm and self-injury, which I discussed in Chapter 1, I decided to use the term NSSI for my subsequent studies.
} 
earlier in this chapter, I was interested in asking people to recall, and reflect on, their most recent episode of NSSI to enable me to hypothesise about the functions of these episodes. Identifying the functions of the NSSI episodes would then allow me to determine whether these descriptions were consistent with the behavioural process of self-injury outlined in the EAM (Chapman et al., 2006).

The questions primarily focused on asking participants to describe what situational, emotional, and cognitive factors had led to their most recent episode of NSSI, how they felt and what they thought about while they were injuring themselves, and whether they had experienced any consequences as a result of the self-injurious behaviour. Participants were also asked about whether they considered their most recent episode of NSSI to be typical of their general pattern of self-injurious behaviour.

Retrospective bias is a limitation of the majority of studies on NSSI but given that I was asking detailed questions about a specific behaviour, I did not want to interview people who had last self-injured years before. In the absence of a definitive boundary of what constitutes current versus historical self-injurious behaviour, I followed the precedent set by other researchers (Brown, Williams, \& Collins, 2007; Whitlock et al., 2008) and invited only those people who had self-injured within the past twelve months to be interviewed.

After being sent the interview questions, two people did not respond to follow-up emails, four people emailed to say that they had decided not to take part in an interview, and 25 people agreed to be interviewed. Unfortunately, due to the small sample size, I was unable to conduct any analyses to determine whether there were significant differences in the types, frequencies, or recency of NSSI between the people who consented and those who declined to take part in an interview.

If people who agreed to be interviewed were engaged in a therapeutic relationship with a mental health clinician, they were to asked to sign a consent form (see Appendix E) giving me permission to inform their clinician about their participation in the study. This requirement was introduced following consultation 
with clinicians who indicated that they would want to know if any of their clients chose to take part. Fifteen people (14 female) reported that they were currently seeing a mental health clinician or that they received mental health support from another professional (i.e., GP, support worker). Out of the 14 people who consented to me contacting their clinician or other support person; one person nominated her GP as her contact person since she was currently engaged in group, not individual, therapy, while another person requested that I contact her support worker. One woman who was engaged in therapy and agreed to take part in an interview did not return her clinician consent form and was not interviewed as a result. The remaining 10 participants were not engaged in therapy or counselling, and, as such, were not required to complete the clinician consent form.

A total of 24 people ( 20 female, 4 male) took part in the interview stage of the study which was conducted over a period of approximately six months. People were invited to bring a support person (e.g., their clinician, a friend, or a family member) with them to the interview; one young woman chose to bring her mother with her. None of the other participants brought a support person to the interview. Additionally, people were given the choice of being interviewed at Victoria University or the mental health service they attended (if applicable). Twenty-two interviews were conducted in a comfortable, private office in the Psychology Department at Victoria University, one interview was conducted at a mental health service attended by the participant, and one interview was conducted at a health service where the participant worked.

Prior to commencing each interview, I reiterated that although I had specific questions to ask, I was interested in learning about any of the person's experiences of NSSI that they wanted to share. That is, the goal of the interview was to understand each person's individual experience of self-injury, while at the same time addressing the research aims. I emphasised that my role as a researcher was to listen to their experiences of self-injurious behaviours; it was not my role to intervene therapeutically unless, as per the standard limits of confidentiality, they were in 
danger of harming themselves or someone else following the interview. Each participant was informed that while I expected the interview to take approximately an hour, we could schedule a second interview if they felt that there was more they wanted to discuss. ${ }^{14}$ Participants were also informed that they could take a break or stop the interview at any time.

I acknowledged that talking about self-injurious behaviours may be distressing and asked whether there was anything I could do to support them if they did become distressed during the interview, or whether they could think of any helpful strategies (e.g., having a cup of tea, taking a cigarette break) to use if they felt upset. ${ }^{15}$ Each person was asked to read through the consent form (see Appendix F) before signing it and given the opportunity to ask questions about the research. On the consent form, people were invited to tick whether they wanted to receive a copy of their interview transcript and/or whether they would like a brief summary of the study results. The majority $(70.8 \%)$ of the participants requested a copy of their transcript and all but one (95.8\%) requested the results summary.

To begin each interview, I asked the person if there was anything that they wanted to talk about first in relation to their self-injurious behaviours or whether they would rather start with the questions I had sent them. This was done in an attempt to give people the space and time to talk about any experiences or issues they felt were relevant to their history of NSSI, rather than immediately focusing on what I wanted to know. The majority of participants opted to begin the interview with the research questions. All of the interviews were audiotaped $\left(\mathrm{M}_{\mathrm{time}}=63\right.$ minutes; range: 29-92 minutes) using a digital audio recorder, the audio files were then uploaded on to a computer, and the original file on the recorder was deleted. The files were stored on a password-protected drive. Using Transcription Buddy V4.0, I transcribed the interviews according to a basic, orthographic notation system,

\footnotetext{
${ }^{14}$ No second interviews were scheduled.

15 This approach was adapted from the University of Washington Risk Assessment Protocol developed by Marsha Linehan and colleagues (Reynolds, Lindenboim, Comtois, Murray, \& Linehan, 2006).
} 
which included all verbal utterances (including repeated and cut-off words) and notable non-verbal utterances (e.g., laughter). ${ }^{16}$ The transcripts, which totalled more than 700 pages, were then checked against the original recordings to ensure accuracy.

At the end of the interview, participants were reimbursed with a Farmers, Warehouse, or Motor Trade Association voucher to the value of $\$ 30$ for taking part and given a list of support organisations (see Appendix C) to contact if they felt distressed. Participants were also invited to contact their clinician (if they had one) or me if they had any concerns. Participants who did not have mental health clinicians, and one participant who was dissatisfied with his clinician, were offered the option of a referral to the Psychology Clinic, which is located in the Department of Psychology at Victoria University. This is a teaching clinic, staffed by two experienced Clinical Psychologists, which is free to students at Victoria and charges up to $\$ 40$ for clients who are not students. Fees were waived for study participants. Participants who declined to accept referrals were informed that they could contact me in the future if they changed their minds. Two male participants requested referrals following their interviews and one female participant emailed me approximately two months after being interviewed to request a referral.

\subsection{Analysing the interview transcripts}

Initially, I attempted to analyse the interview transcripts thematically (Braun \& Clark, 2006), but found that identifying group-level themes across the data corpus precluded an individual-level analysis of the temporal process of each person's most recent episode of NSSI. Rather I needed an analytic method that would allow me to examine how each episode had unfolded over time. Drawing on the behaviour analysis literature, I developed a qualitative method called Interpretative Functional Analysis. Before outlining the rationale, epistemological assumptions, and steps of

\footnotetext{
${ }^{16}$ My choice of transcription conventions reflects my interest in what participants said rather than how they said it, which precluded the need to transcribe linguistic details such as the timing of pauses and syllable lengths. Words in single brackets signify my best interpretation of the word(s). The word unclear in single brackets signifies my inability to interpret what was said.
} 
this method in detail, it is first necessary to discuss the theoretical underpinnings and practical limitations of conducting functional analyses with typically developing populations.

\subsubsection{What is a functional analysis?}

As an idiographic approach to the assessment and treatment of problem behaviours, a functional analysis involves examining how a person's behaviour varies according to their unique learning history and environment (Farmer \& Latner, 2007). The aim of a functional analysis is to establish which contingencies are maintaining a person's problem behaviour so that the associated antecedents and consequences can be targeted to decrease the behaviour (Hanley, Iwata, \& McCord, 2003). Accordingly, the three phases of a functional analysis involve: (1) gathering information about the problem behaviour and variables that may impact on that behaviour, (2) hypothesising about what contextual variables cause and maintain the behaviour to determine the function of the behaviour, and (3) manipulating particular variables (i.e., antecedents and consequences) in an attempt to alter the behaviour (Cone, 1997).

\subsubsection{Utilising functional analyses with typically developing populations}

Functional analyses have been the mainstay of therapeutic assessment and intervention with developmentally disabled populations since the 1960s, but this approach has only more recently been applied to typically developing populations with the advent of Clinical Behaviour Analysis (Anderson, 2007; Dougher \& Hayes, 2000). Originating in the 1990s, Clinical Behaviour Analysis can be understood, in part, as a reaction against the structuralist system used to assess, diagnose, and treat mental illness (Dougher \& Hayes, 2000).

From a structuralist perspective, problem behaviour is perceived as an indicator of underlying pathology; as such, causes of behaviour can be found within people (Follette, Naugle, \& Linnerooth, 2000; Sturmey et al., 2007a). In contrast, functionalists maintain that behaviour is an adaptive response to the environmental context and, as such, causes of behaviour lie within the environment (Sturmey et al., 
2007a). Clinicians working within a behavioural paradigm view functional approaches as non-pathologising compared to structuralist approaches, because the target for change is the environment rather than the person (Sturmey et al., 2007a).

Structuralism, however, is privileged over functionalism within the domain of mental health research and clinical practice because of the hegemony of the DSM (American Psychiatric Association, 2000). Classifying problem behaviours on the basis of shared topographical features has advantages: it provides a shared, common language for clinicians; indicates which behaviours are likely to covary; and points to possible interventions (Nelson-Gray \& Farmer, 1999).

However, structural approaches to psychopathology also have a number of disadvantages. As a syndromal classification system, the DSM-IV-TR (American Psychiatric Association, 2000) provides insight into what a person has but not how they interact with the environment and failing to identify the functions of problem behaviours makes it more difficult to alter these behaviours (Cipani \& Schock, 2007; Cone, 1997). Given that one behaviour can serve many functions and a single function (e.g., escape) can maintain a range of topographically dissimilar behaviours, functional classification systems (see Cipani \& Schock, 2007) arguably have more utility than syndromal classification systems when it comes to understanding problem behaviours (Cone, 1997).

Clinical Behaviour Analysis (CBA) is informed by Applied Behaviour Analysis (ABA) in that it involves "the application of the assumptions, principles and methods of modern functional contextual behavior analysis" to mental disorders (Dougher, \& Hayes, 2000, p. 11), but it also has three key differences:

(1) ABA focuses on external stimuli that can be observed (Kohlenberg, Tsai, \& Dougher, 1993); CBA focuses on intrapersonal stimuli (e.g., thoughts, emotions) because these internal processes ${ }^{17}$ are extremely relevant to the development and maintenance of psychopathology (Hayes et al., 2006).

\footnotetext{
17 Within the field of radical behaviourism, private events such as thoughts, emotions, and somatic sensations qualify as behaviours that can be functionally assessed (Cuper, Merwin, \& Lynch, 2007).
} 
(2) To gather information about problem behaviours, applied behaviour analysts typically depend on direct observations (Hanley et al., 2003). In contrast, clinical behaviour analysts working within mental health settings are seldom able to observe problem behaviours because they are internal processes or they occur outside of therapy (Farmer \& Latner, 2007; Miltenberger, 2005). Consequently, self-report is an important source of information utilised by clinical behaviour analysts (Miltenberger, 2005).

(3) Applied behaviour analysts manipulate specific variables to test whether or not they reinforce the problem behaviour (Kohlenberg et al., 1993), whereas clinical behaviour analysts are typically unable to manipulate variables that may reinforce problem behaviours as these are often internal (Miltenberger, 2005).

\subsubsection{Interpretative Functional Analysis}

\subsubsection{Rationale}

Given that the overarching research question in this thesis is whether NSSI functions primarily as an experientially avoidant behaviour within Aotearoa New Zealand, it was necessary for me to assess whether the descriptions of specific selfinjury episodes aligned with the operant process described in the EAM (Chapman et al., 2006). My interview questions were designed to elicit the antecedents and consequences of each person's most recent episode of self-injurious behaviour and, as such, assessing these descriptions in order to hypothesise the function(s) of the behaviours appeared to be the most appropriate form of analysis.

To analyse the interviews, I developed a method called Interpretative Functional Analysis which was informed by the principles of CBA. Although I have named this method Interpretative Functional Analysis ${ }^{18}$, it does not meet the

\footnotetext{
${ }^{18}$ Although I functionally assessed, rather than analysed, each episode of NSSI, I chose to call my method Interpretative Functional Analysis, not Interpretative Functional Assessment, because I went beyond the realm of individual assessment to then compare the antecedents and consequences of NSSI episodes both within and between functions.
} 
requirements for a full functional analysis as defined within CBA because I was unable to work therapeutically with people to bring about changes in their behaviours. As discussed earlier, a functional analysis consists of three phases: gathering information about a problem behaviour, developing a hypothesis about the function(s) of that behaviour, and manipulating relevant variables in an attempt to decrease or eliminate the behaviour (Cone, 1997). I only conducted one interview with each person in a research rather than therapeutic context; accordingly, I did not complete the treatment phase of a functional analysis.

Instead, I focused on the first two phases of information gathering and hypothesis formulation, collectively called a functional assessment (Cipani \& Schock, 2007; Cone, 1997), the outcome of which is a "hypothesis statement identifying environmental variables that likely evoke and maintain the behavior of concern" (Anderson, 2007, p. 459). Assessing the context in which the self-injury occurred (e.g., the antecedents) in conjunction with the consequences that followed the behaviour, allowed me to hypothesise about the functions of participants' NSSI (Cone, 1997).

Once I had formulated a set of hypotheses about the functions of each selfinjury episode, I compared the hypothesised functions across the group of participants to identify similarities and differences between antecedents and consequences both within the same function and across different functions. In sum, the aims of conducting an Interpretative Functional Analysis were to: (1) assess each person's most recent episode of NSSI to derive a hypothesis statement about the function(s) of their behaviour and (2) compare the antecedents and consequences of the self-injurious behaviours both within and between the different functions.

Functionally assessing discrete episodes of self-injury to compare behavioural functions across a group of research participants is unorthodox. Certainly, functional analyses of problem behaviours within typically developing populations have been published as case studies (e.g., Farmer \& Latner, 2007) and within treatment manuals (e.g., Linehan, 1993a), but I have been unable to identify any studies where 
these individual analyses were then compared at a group level. However, I believe that the form of analysis I developed for this study was the most appropriate method to answer my research question.

\subsubsection{Epistemological assumptions}

I included the word 'interpretative' when labelling my method as an explicit recognition of the impact of my values and perspectives when writing the interview questions, interviewing the participants, transcribing the data, formulating hypotheses about the functions of the behaviours, and writing up the results. Each of these phases involved differing levels of interpretation, all of which were informed by my own personal epistemologies, values, and understanding of the self-injury literature. I concur with Angen (2000) that:

Truth, from an interpretive perspective, is no longer based on a one-to-one correspondence to objective reality. It is acknowledged that what we can know of reality is socially constructed through our intersubjective experiences within the lived world, which results in a form of truth that is negotiated through dialogue. (p. 386)

Additionally, the absence of a treatment phase in the functional analyses I conducted prevented me from testing my hypotheses, which instead remained as functional interpretations.

Arguably the most important epistemological assumption underlying Interpretative Functional Analysis, which I touched on when comparing ABA to CBA, is that CBA considers self-report to be a valid form of data. While applied behaviour analysts privilege independent observation and dismiss self-report as an unreliable and invalid approach to assessing behaviour (Cipani \& Schock, 2007), clinical behaviour analysts maintain that it is possible to identify reinforcers through self-report while remaining cognisant of the limitations associated with this method (Sturmey, Ward-Horner, Marroquin, \& Doran, 2007b).

\subsubsection{Coding system}

The three term contingency of antecedent-behaviour-consequence formed the basis of my coding system, but I also took into account relevant historical antecedents that provided insight into each person's current episode of NSSI. 
Behaviours were then categorised according to whether they served an escape/avoidant or access function (Cipani \& Schock, 2007).

\subsection{Relevant learning history}

Historical variables are relevant to completing a functional assessment if they impact on, or point to, significant, contemporary antecedents that are amenable to change (Farmer \& Latner, 2007; Follette et al., 2000). Since historical events (e.g., trauma) can have an impact on current behaviour and each person's learning history determines which behaviours are maintained over time (Sturmey et al., 2007b), I extracted historical information from the interview transcripts that I interpreted as relevant to participants' most recent episode of NSSI. However, it should be noted that it is often difficult to determine the extent to which these antecedents impact on current behaviour, especially when the information about how the behaviour functions is gathered through retrospective self-report.

\subsection{Antecedents}

Identical behaviours can fulfill different functions depending on the antecedents of those behaviours (Cipani \& Schock, 2007); consequently, it is possible to infer the functions of a behaviour by identifying the antecedents (Stickney \& Miltenberger, 1999). Temporally remote antecedents can function as setting events which then have an effect on more immediate antecedents (Stickney \& Miltenberger, 1999). For example, receiving a low grade for a report might lead someone to question their academic abilities and subsequent thoughts of inadequacy and failure then lead to an episode of NSSI.

Two types of antecedents were identified in the interview transcripts: Establishing Operations (EO) and Discriminative Stimuli (SD). Establishing operations are conditions (e.g., deprivation, aversive stimulation) that establish particular consequences as reinforcers (Dougher \& Hackbert, 2000; Follette et al., 2000); people are motivated to engage in specific behaviours because of EOs (Follette et al., 2000; Miltenberger, 2005). To identify an EO, a useful question to ask is: "Why does the person "want" this consequence?" (McGill, 1999, p. 399). When a person 
engages in self-injury to gain the consequence of relief, it is likely that they wanted that relief because they were experiencing aversive internal stimulation (e.g., anxiety, negative thoughts). In this instance, the aversive internal stimulation would be classified as an EO because it establishes the relief as a reinforcer for self-injury.

Establishing operations have a value-altering effect in that they determine how effective or potent a particular reinforcer will be in a specific situation (Laraway, Snycerski, Michael, \& Poling, 2003; Michael, 1993; Miltenberger, 2004). A reinforcer's value thus shifts along a continuum depending on the intensity of the EO (Laraway et al., 2003). For example, food deprivation is an EO but people can be more or less food-deprived. The more hungry a person is, the more likely it is that food will be an effective reinforcer. Establishing operations also have a behaviour-altering effect in that they increase the likelihood of the person engaging in behaviours that are typically followed by that reinforcer (Laraway et al., 2003; Michael, 1993; Miltenberger, 2004).

The second type of antecedents, SD's, signal the likelihood that a behaviour will elicit a particular consequence because the stimulus has typically been present when that behaviour was reinforced in the past (Farmer \& Latner, 2007; Kearney, 2008). The presence of a $S_{D}$ (e.g., NSSI implements) therefore signals to the person that there is an opportunity for reinforcement or, in other words, the likelihood that a particular reinforcer is available (Miltenberger, 2005).

Since a specific behaviour can be triggered by a number of different $S_{D}$ 's (Cuper, Merwin, \& Lynch, 2007), the capacity of stimuli to function as reinforcers depends on the EOs. Discriminative stimuli therefore work in conjunction with EOs to bring about particular behaviours. An SD will only impact on behaviour when an EO is present because a person has to want a particular consequence in order to be to be influenced by the opportunity to experience that consequence (Laraway et al., 2003). For example, a razor blade may have been established as an SD in that it signals the opportunity for the release of intense anger following cutting behaviour. If the EO of intense anger is absent, it is unlikely that the person will use the razor blade to cut themselves because they will not be motivated to seek relief. 


\subsection{Target behaviour}

Since the focus of my analysis was the functions of people's self-injury, not the topographical features of these behaviours, I did not purposefully seek specific details beyond asking how they had hurt themselves and, occasionally, how many times they had hurt themselves during their most recent episode. Some participants did chose to volunteer detailed information about how they had injured themselves and the implements they had used for this purpose, while others provided little information about the actual self-injurious behaviour.

\subsection{Consequences}

Particular behaviours are selected for through operant conditioning; that is, behaviours impact on the environment to produce consequences and are subsequently more or less likely in particular contexts because of those consequences (Cipani \& Schock, 2007; Sturmey et al., 2007b). The likelihood of a behaviour reoccurring depends on whether an individual views the particular consequences of that behaviour as positive or aversive. Positive consequences maintain or increase behaviour, whereas aversive consequences temporarily or permanently suppress behaviour (Goldfried \& Sprafkin, 1976).

There are four general categories of consequences - positive reinforcement, negative reinforcement, positive punishment, and negative punishment (Sturmey et al., 2007b) - all of which can be intrapersonally or interpersonally mediated. As a result, I coded for four categories of reinforcement: intrapersonal negative reinforcement, intrapersonal positive reinforcement, interpersonal negative reinforcement, and interpersonal positive reinforcement. I coded aversive consequences (i.e., punishers) more generally because when considering clinical behaviour problems (e.g., substance abuse), the aversive consequences are usually delayed and so do not function to suppress the behaviour (Goldfried \& Sprafkin, 1976). For example, aversive consequences following NSSI, such as others' reactions to scarring, are typically delayed compared to positive consequences, such as an adrenalin rush or relief of emotional distress, which are immediate. 


\subsection{Escape or access: Functions of the target behaviour}

The function of a behaviour refers to the way in which that behaviour impacts on the environment (Hanley et al., 2003). As summarised by Cipani and Schock (2007), behaviour serves two overarching functions: to escape or avoid negative events and to access positive events. Escape or avoidance behaviours are maintained via negative reinforcement, while access behaviours are maintained via positive reinforcement (Cipani \& Schock, 2007). Furthermore, behaviours can be intrapersonally or interpersonally mediated (Cipani \& Schock, 2007). Each episode of self-injury therefore was coded according to whether it functioned as intrapersonal or interpersonal escape/avoidance or access.

\subsubsection{Steps in the Interpretative Functional Analysis}

In order to complete the Interpretative Functional Analysis, I followed the seven steps outlined below:

(1) I read through all of the interview transcripts to identify any extracts that could be relevant to each person's most recent episode, including their learning history, any experiences that motivated them to self-injure, the antecedent events or circumstances that occurred before they self-injured, descriptions of the target behaviour, and the consequences that followed that behaviour. I also identified any information related to whether they considered their most recent episode to be typical of their general pattern of self-injury.

(2) The relevant extracts from each interview transcript were then cut and pasted into a word document for each person under the following headings: relevant learning history, establishing operations, discriminative stimuli, target behaviour, consequences, and typicality of episode. Any surrounding, contextual information that was important in order to understand and interpret the extract was retained, including any of my questions or comments that led to particular explanations or elaborations from participants. 
(3) Once tabulated, I read through the selected extracts for each person and summarised any information from the extracts that I deemed relevant to a functional assessment of each person's most recent episode of NSSI.

I then completed schematic representations (c.f., Braun \& Clarke, 2006) of the behavioural process of each person's most recent episode of self-injury (see Figure 3 for an example). The structure of these diagrams was adapted from those presented in Follette et al. (2000) and Farmer and Latner (2007). The black circle represents the probability that the discriminative stimuli and establishing operations evoked the target behaviour in the context of the relevant learning history.

(5) An iterative and reflexive process was followed whereby I re-read the extracts to check that I had summarised all of the information that was relevant to completing a functional assessment of each episode and that I had included all of this information in the diagrams. I also re-read the original transcripts to ensure that extracts and the diagrams represented each person's most recent episode as they had described it and that I had not inadvertently left out pertinent information.

(6) Based on the information depicted in the schematic representations, I formed a hypothesis as to the primary function of each person's most recent episode of self-injurious behaviour in the context of their relevant learning history. For example, I hypothesised that Melanie's $\mathrm{s}^{19}$ most recent episode of NSSI (see Figure 3) functioned as an escape from aversive thoughts and emotions.

(7) The hypothesised functions were then compared across participants to identify any similarities or differences in the antecedents and consequences of the NSSI episodes within the same function, and to identify any similarities or differences between functions.

${ }^{19}$ All of the participants were given pseudonyms to protect their identities. 


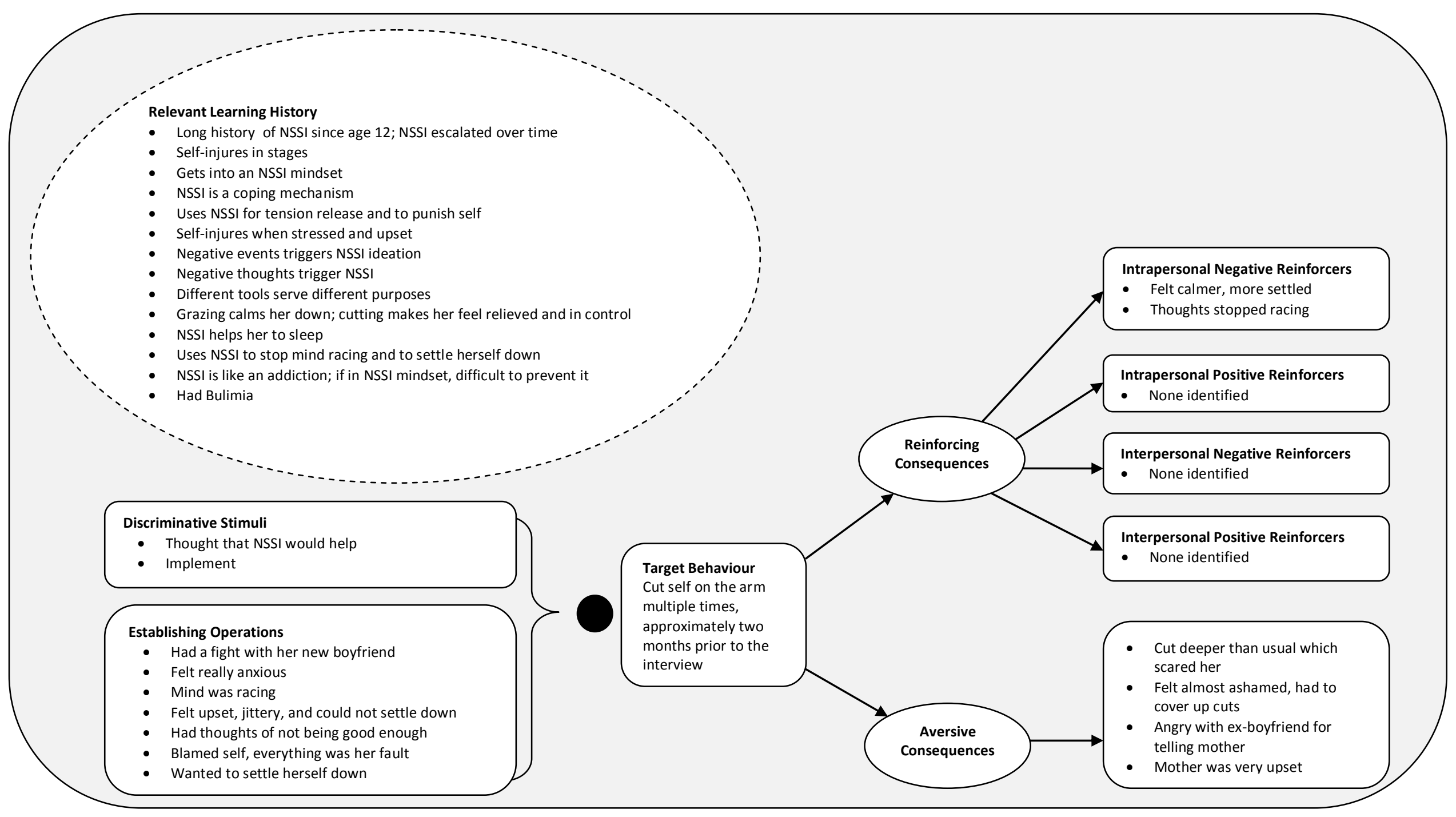

Figure 3. Functional schematic of Melanie's most recent episode of NSSI. 


\subsection{Interview results}

Of the 24 people who were interviewed, one participant provided too little information about their most recent episode for a functional assessment and three participants did not identify experiencing any form of reinforcement following their self-injurious behaviour. As a result, only 20 episodes of self-injury are included in the following analysis. Figure 4 shows which participants' episodes were hypothesised to fulfil each function. The colour purple is used to specify participants whose episode was hypothesised to only fulfil one function, blue specifies participants whose episode fulfilled two functions, and green specifies participants whose episode fulfilled three functions.

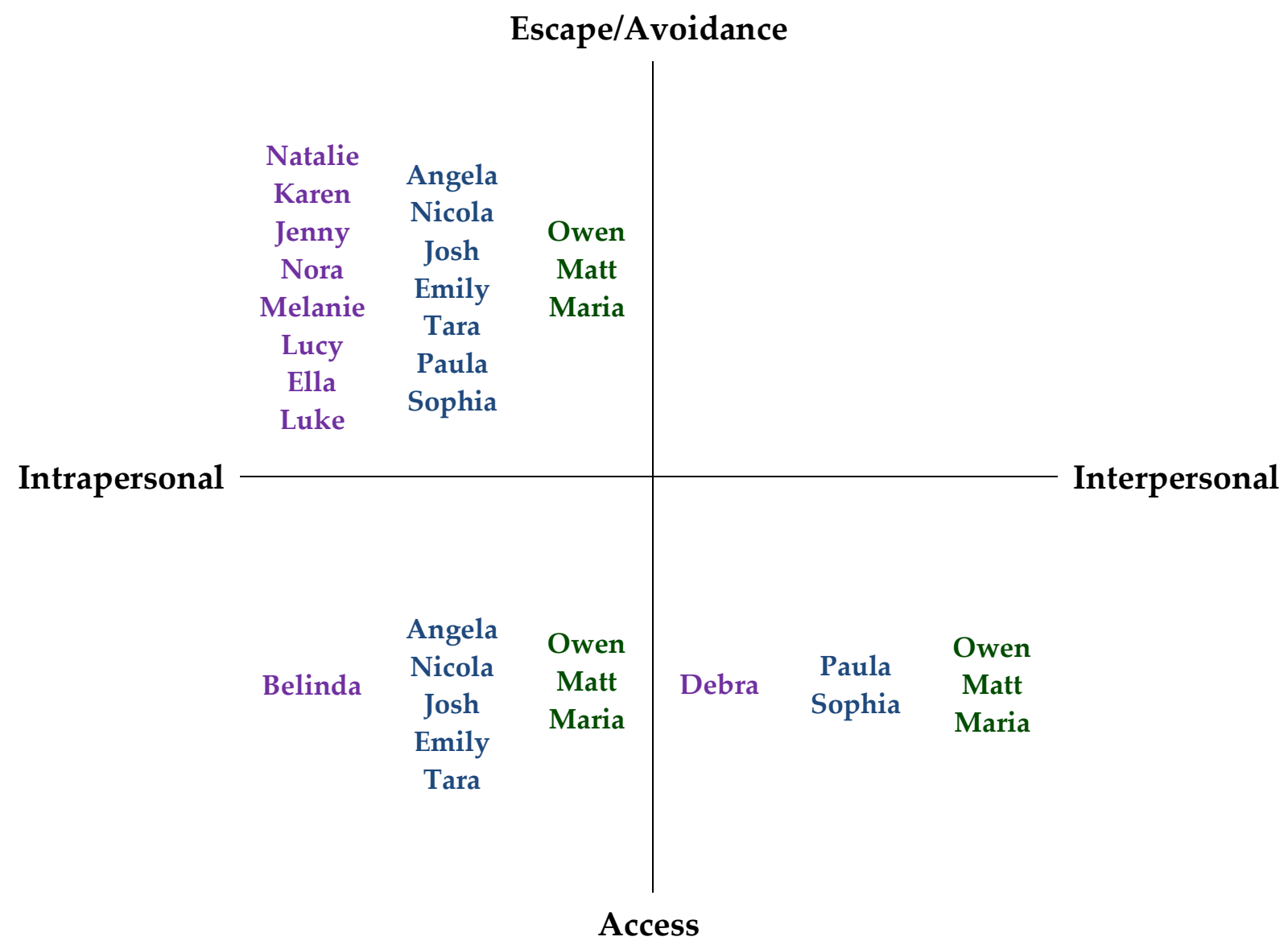

Figure 4. Functions fulfilled by each participant's most recent episode of NSSI. 
Although the most commonly identified function was intrapersonal escape/avoidance, many episodes appeared to serve multiple functions. Of the episodes that only served one function, eight were categorised as intrapersonal escape/avoidance, one was categorised as intrapersonal access, and one as interpersonal access. Furthermore, as is evident from Figure 4, no episodes were hypothesised to serve an interpersonal escape/avoidant function.

In the following sections, I present the detailed findings of my Interpretative Functional Analysis, using relevant interview extracts to illustrate my arguments. The EOs, Sn's, and reinforcing consequences identified within the transcripts for each of the three functions - intrapersonal escape/avoidance, intrapersonal access, and interpersonal access - are discussed. I also briefly examine two historical examples (i.e., not the most recent episode) where self-injury functioned as a form of interpersonal escape/avoidance. Finally, I discuss the aversive consequences described by participants, which may have functioned to punish their most recent episode of self-injurious behaviour.

\subsubsection{Intrapersonal escape/avoidance}

When I considered the antecedents and consequences of the NSSI episodes within the context of each person's learning history, I hypothesised that the majority of these episodes $(N=18)$ had functioned as a form of escape from aversive emotions and thoughts. People described an array of both distal and proximal negative events which, along with negative intrapersonal experiences, had established the motivation for them to engage in NSSI. They also identified a variety of stimuli that had signalled to them the potential for negative reinforcement following selfinjurious behaviour. All of these participants reported that their episode of selfinjury was followed by the reduction or elimination of unwanted emotions and/or thoughts.

Although it is difficult to be certain that the distal events reported did have an effect on people's most recent episode of self-injury, in some cases it is likely that these events partly established the conditions for self-injury to function as a form of 
escape or avoidance. For example, one participant reported experiencing flashbacks before self-injuring and two participants mentioned traumatic events that they had not fully come to terms with. It is probable that the consequences of these events influenced their most recent episode of NSSI.

\subsubsection{Establishing Operations}

Emily first cut herself on purpose at the age of 11 and had since self-injured in stages, while concurrently struggling with depression. Her self-injury, however, got "a lot worse" after her marriage ended; she attributed this escalation and her most recent episode of NSSI to the stressful transition she was going through at the time:

Emily: I think the type of stress I was experiencing was different...um it was a it wasn't stress and anxiety directly related to to you know a um a clinical depression it was...it was related to $\mathrm{n}$ - to a trauma in my life you know...to to my husband leaving me...my life was ((laughing)) cha- it was changing massively...um you know during that especially that six months...I felt like it was changing for the better...um but it didn't make the the transition ((laughing)) ((laugh)) easy you know ${ }^{20}$

Maria had similarly experienced traumatic loss and her grief, following the unexpected and untimely death of a friend, most likely served as a distal EO for her most recent episode of NSSI. Although she recalled self-injuring between the ages of approximately 11 to 14 by banging her head against things and scratching herself, she had not continued to self-injure through her later adolescence. Indeed, she had only begun hurting herself again after her friend's death.

At the time of her most recent episode of NSSI, Maria was also supporting her flatmate who had been the victim of a rape and attempting to focus on a very difficult year of university studies. Although these stressors, from what she described as "the year from hell", combined to establish the conditions where she began self-injuring again, it is likely that only the loss of her friend functioned as a distal EO for her most recent episode of cutting because her grief remained unresolved:

Maria: $\quad$ I think it started out of my frustration with my friend and not...just with her but with the

${ }^{20}$ The ellipses in this and all following interview extracts signify deleted text. Text was deleted when it was considered extraneous to the points being discussed. Standalone minimal encouragers (e.g., $\mathrm{mhmm}$, yeah) were deleted as these disrupt the readability of extracts. 
situation she was in...but I think what it really was for me...was the the grief of my friend dying...I think that was that was the huge thing 'cos it was such a hard thing to grasp

Although the effect of these distal EOs on both Emily and Maria's subsequent episodes of NSSI cannot be conclusively determined, these events did appear to exert at least some influence over their engagement in their most recent episode of self-injurious behaviour.

Along with distal events, participants talked about how specific proximal events or ongoing stressors, followed by the experience of negative emotions and thoughts, had motivated them to seek relief or release through self-injury. The events and conditions that served as EOs were typically unique to each person and included: fighting, or breaking up, with a boyfriend; being left alone at home without family for two weeks; experiencing an upsetting therapy session; moving back to Wellington; failing at university; ongoing physical and mental health issues; and changing to a different anti-psychotic medication.

Each of these EOs was typically accompanied by intense, negative emotions and thoughts which culminated in self-injury. The accumulating impact of aversive intrapersonal experiences was summed up by Owen who described what led to his most recent episode of self-injurious behaviour, where he burnt himself on the hand multiple times, as follows:

Owen: the reason I did it recently was because um was because I thought I was getting ill again...I thought I was getting unwell um I was on a med change and it wasn't going particularly well... and um I just didn't wanna um go through that again and and I felt like I was yeah useless and this was going to be the end of my life ra ra rarara ((sniffs)) um and so I um yeah so I just I d- I I just completely broke down really...and um just started to to do that

When I asked Owen what indications he had that he was becoming unwell again, he identified that he thought he had heard a voice and had experienced "raw anxiety that is just really paralysing." One of the reasons becoming unwell again was so frightening for him was because of his previous psychotic episodes which he described as an "open wound":

Owen: $\quad$ if I refer to it people think ah why is he still talking about that why does it what does it matter...it was years ago you move on blah blah blah blah they don't understand that it 
was such a big trauma

Potentially hearing a voice in the context of a medication change reminded Owen of the trauma of his previous two psychotic episodes. These memories were accompanied by intense anger at the unfairness of the situation, self-hatred, and selfblame because Owen thought he had partly caused his Schizophrenia by smoking marijuana and partying too hard during adolescence:

Owen: it it just didn't seem fair...and I just hated myself because because for a lot of like it also links into the fact that I think um to a degree that I was a player in my illness...I dunno if I caused it but I definitely made it worse um so there was that feeling that because it's been a real tough four or five years and so it's the realisation that the uh you know the feeling that this is going to be like this for the rest of my life...um and that I've done it to myself

Given his past learning history, it is understandable that Owen chose to burn himself in the context of such intense, negative emotions, self-recrimination, and catastrophising about what the future held for him. After all, a long, established pattern of self-injury since the age of 13 years old had reinforced for him that selfinjury was "the answer" to his self-hatred and depression:

Owen: when you hate yourself that much or when you're that messed up and you're that down...the feeling of pain like just associated with with um the way you're feeling seems it seems justifiable and it seems the way to do it...it seems the answer...it just feels like sort of what you deserve as well...it's kind of weird but it feels like it's what you what you um what you what what would feel the best for you at that time is to feel in pain

Although some of the EOs experienced by Owen were unique to him and motivated him to self-injure because of his particular learning history (i.e., the possibility that he had heard a voice was especially anxiety-provoking because of the trauma associated with his previous psychotic episodes), other EOs, such as the negative emotions and self-denigrating thoughts that he experienced, were similarly described by many of the participants.

Anger, frustration, self-hatred, and self-blame were some of the most common emotions discussed in the interviews as EOs for the self-injury episodes and, more often than not, were accompanied by a range of self-critical thoughts: Ella: I was just feeling really frustrated and really really overwhelmed and really angry at my body...for um not working the way ((laugh)) it's meant to I guess 
Nora: I think the biggest thing was I was um angry with who I was... and I didn't feel like I fit anywhere....in that I didn't really have a place in the world...I just didn't really like anything about myself or the situation that I was in...um and just thinking about um if people really actually did like me or if it was all fake...um and if my parents loved me

Lucy: I was just really upset and decided everyone hated me and stuff like that...the stuff that triggered it was all just to do with kind of rejection and stuff like that...I was angry at the fact that what seemed like little insignificant things were still affecting me

Robyn: $\quad \mathrm{mhmm}$ so when you're feeling like that what kind of thoughts run through your head

Lucy: $\quad(($ laugh $))$ not very nice ones ((laughing))...useless worthless just stuff along those lines ((laugh))

Tara: I was kinda frustrated with myself because...I'm not normal and I just wanted to kind of be better and fine and that was all and so I dunno it was like a well it kind of was a way of getting over that anger and frustration at myself by doing that...I think it was quite a hateful thing to do to myself

Josh: I guess the main thing that really sort of triggered off me actually um cutting was uh it's kind of a sort of disgusted at my own thoughts at the time... about how I was um sort of reacting to things at the moment... as far as uni went and um like that I'm not actually really trying at ((laugh))...at anything ((laughing))...I was just sort of uh trying to figure out why I was doing that which led onto thinking back to all sorts of ((clears throat)) past instances where I've been doing something and then kind of given up and then...um yeah sort of got quite angry at myself for that and um yeah just kind of got a bit disgusted with the fact that I was ((laughing)) doing it um which sort of turned into thoughts about being sort of useless... and uh not actually sticking anything through

Paula: um I think the main thoughts that were going through my head were um the areas that um have triggered my eating disorder for instance...um neglect from parents (unclear)...um disruptive childhood and um certain events that have happened...to me like um abuse and stuff like that and so those sort of things replay in my mind and...um then like the the thoughts of how much I hate myself

Sophia: I think I probably just felt quite exhausted...um and um frustrated at feeling like I wasn't coping...I think I felt angry...um directed at myself um yeah and

Robyn: when you feel angry like that about yourself...um what kind of thoughts would go through your head in relation to to that anger...

Sophia: $\quad$...I feel frustrated that well it feels like to me that little things are affecting me....and I feel frustrated that I that even though I don't want them to that they do...um and I think that probably leads to me thinking that in general I don't cope very well...um as a person and that you know and and doubting whether how competent I am as a mum...um and how supportive I am as a wife and it it yeah it just I think it just brings into question lots of those things that I um put my self value on

The combined pressure of intense, high arousal emotions such as anger and frustration, along with thoughts about being useless and worthless, led to people feeling overwhelmed and desperate to alleviate their distress. Ella captures how 
intensely she felt prior to self-injuring in the following description of her reaction to ongoing health problems:

Ella: $\quad$ I was quite indignant like how dare you ((laughing))...you know how dare you put me through this shit and... and why aren't you doing what you're 'sposed to and why won't you work and $\mathrm{w}$ - what the fuck is wrong with you kind of...so it was very um yeah it was al- almost as if I had taken kind of every shit experience in my entire life and compounded it into like hatred at this one particular area

Another participant specifically linked her episode of self-injury to the intensity of her anxiety which manifested itself both psychologically and physically:

Robyn: mm ok um so er can you describe how you were feeling before you cut yourself

Melanie: um really anxious my mind was racing like it would take tiny little ideas...and just run off with them absolutely no reason.... and yeah

Robyn: yeah yuh so you're feeling really anxious what else were you feeling

Melanie: well I was quite upset considering...everything that was going on and...I think I was crying quite a bit and just really jittery and couldn't settle myself down at all

When I asked Melanie about the content of her racing thoughts, self-blame and worthlessness comparable to that experienced by other participants appeared to dominate:

Melanie: that I wasn't good enough I don't know it was just my mind was racing so much I don't really know what I was thinking...I was just all over the place all sorts of thoughts about not being good enough and...that everything was my fault sort of...jumping into my head ((laughing))

Melanie explained that in the years that she has been hurting herself, self-injury has developed into a coping mechanism which she uses to regulate her emotions. Thus in describing her motivation for her most recent episode of cutting she simply said: "I wanted to settle myself down".

All of the emotional EOs (e.g., anger, frustration) discussed so far could be categorised as negatively valenced, high arousal emotions. These emotions were typically self-referential and accompanied by negative, self-castigating thoughts. In addition to these high arousal emotions, some people also described experiencing negatively valenced, low arousal emotions (e.g., feeling down or hopeless) prior to their episode of NSSI, while others exclusively identified experiencing low arousal, negative emotions: 
Ella: I was feeling really just self-destructive and just overwhelmed an- and really really hopeless

Karen: $\quad$ I just got more and more overwhelmed and depressed...I was feeling really useless...I've just come back down to Wellington to see all my friends and they're all really successful and have good jobs now and I've come back and I'm just a bum and I'm not doing anything and I'm stressing out 'cos I can't pay my bond and ((laughs))...just um it seemed like everybody was on top of everything and I was just slowly drowning in it all ((laughing))

Nicola: I think it was just like even just a day that I was just feeling like real...kind of empty...like just a day that I was feeling useless pretty much

Paula: I remember how I was feeling and stuff like that...so I think that was just me so in-depth with being overwhelmed by being so depressed and stuff that I...I sort of felt dead...like I couldn't feel anything

Emily: it was still one of those things of those moments of getting stressed out and um feeling really down and and and fragile

Natalie: I was feeling really really low...I was just alone for two weeks...I dunno it kinda just got overwhelming...I kind of felt like everyone had abandoned me ((laugh))...I kind of thought that I dunno maybe it was my fault they had gone away and I kind of blow things up in my head ((laughs))...it's very annoying...so everyone was gone and then I was like ah it must be my fault...I guess it made me sad...that everyone was gone and then when I get sad I kind of withdrawal into myself withdraw... whatever the word is ((laughs)) and I kind of just feel like I'm an empty shell walking around

Additionally, two participants, who were depressed at the time of their most recent episode of NSSI, acknowledged thinking about suicide prior to cutting themselves. Angela who described herself as having been in a "really bad episode of depression for a long time" and largely house-bound because of her intense social anxiety, explained how her suicidal ideation was linked to feelings of hopelessness and self-stigmatising thoughts:

Angela: I didn't self-harm intending to kill myself but I um had been thinking about suicide and things like that...so um with those yeah just all of that sort of going round in my mind that I wouldn't be able to um live a a normal um normal life sort of have have a job do all... of the normal things sort of having the um thought that I'm not doing the things that most eighteen year olds should be doing...um and yeah just feeling very hopeless and uh not really seeing an end an end to it

For the participants who identified thinking about suicide prior to self-injuring, it is possible that suicidal ideation was one of the EOs they were motivated to escape from through hurting themselves. 
From people's descriptions of what led up to their most recent episode of NSSI, it is clear that they had to contend with multiple EOs. None of the participants identified only a single event, emotion, or thought as the trigger for their self-injury but rather explained how numerous aversive experiences, what Luke described as "layers of stress", established the conditions for their self-injurious behaviour to function as a form of escape/avoidance. Sophia summed up the emotional and cognitive maelstrom that preceded her episode of NSSI as follows:

Sophia: my head's just going so fast and...just knowing that I'm getting stressed or angry at you know but that my emotions are out of control and not... and not $\mathrm{f}$ - feeling like I can um rein them in...having that sense of um yeah yeah kind of a desperateness because you just feel like it's just never gonna stop...like I just wanna go to sleep I've got you know I've got enough to do tomorrow as it is and you know there's all this stuff and I should j-not even be thinking about it but uh it's just gonna it's just gonna get out of hand

Her perception that the pressure would keep building up resonated with a metaphor used by two of the participants in relation to their general experiences of self-injury (i.e., not their most recent episode). They explained how one aversive experience would snowball into other negative thoughts and emotions, growing in size until the magnitude of the situation became overwhelming.

Ella had a different way of explaining the mounting pressure of her frustration, stress, and anger; to her, it was analogous to leaving a lid on a pot of boiling liquid:

Ella: $\quad$ I use the boiling pot analogy where if you leave the lid on a pot... and it just boils up and then...it just if you don't let a bit of steam out it explodes and shit hits all over the kitchen you know

Before her most recent episode of self-injury, Ella described herself as wracking her brain to come up with a solution to prevent the impending explosion:

Ella: I was almost kind of desperately thinking you know I just need to do something and if I do something...it I'll feel better

In her self-described quest for "something to kind of take the edge off", she considered smoking (even though she had given up approximately a year and half before), overdosing on a bottle of sleeping pills, or drinking a bottle of bourbon, but finally settled on self-injury. 
Although Ella was the only participant who reported actively weighing up alternatives to self-injury, she was not the only one who tried problem-solving strategies. Nicola attempted to talk herself out of self-injury while Lucy went for a run to try to calm down. Unfortunately, neither of these participants was able to prevent themselves from engaging in self-injury. When I asked Lucy if going for a run usually helps, she replied: "Sometimes...but ((laugh)) it's more just kind of preventing the inevitable ((laughing))".

The perception that self-injury was inevitable when people surpassed a particular threshold of distress was reported by several participants. Once the negative emotions and thoughts became overwhelming, a type of cognitive constriction (Schneidman, 1996) occurred where self-injury was judged by the participants to be the only solution that would result in relief:

Nora: I did wanna stop but I knew that it was all I had at that time

Lucy: $\quad$ I was just really upset mm I dunno once it's in my head that that's how I'm gonna deal with it I kind of can't stop it

Emily: I kind of get to this point and I know that I was gonna do that...you know and that doing that would mean that I could go to sleep afterwards...even though I know there's gonna be a fall-out from it over the next few days over the next week but I felt like I was getting better ((sighs)) you know so I felt like well I'll be able to deal with it by then but right now I can't... and I need I need to do this

This constricted cognition was exemplified by what two participants referred to as their self-injury mindset. When Owen was in the mindset, self-injury became compulsive and the "right thing to do":

Owen: I would self-harm with whatever um by whatever means...um if I was in that state of mind...you're in that mindset that it's the only thing to do...and and that that um you deserve it and that you think of all the bad things that have happened and that you think you've been the cause of all these bad things... and so it seems like the the right thing to do

Owen's belief that self-injuring was the "only thing to do" was echoed by Melanie who described that she would get into the "mindset of thinking that every time something went wrong", the solution was to cut herself.

Similarly, Nora attributed her inability to prevent her most recent episode of self-injury to the "headspace" she was in: 
Nora: and I I was kind of like looking back on like you didn't need to make such a big deal out of it but I know at the time it was just the headspace I was in and that was all I could do to... kind of get through...I knew that it would make me feel better at least for a little bit it would make all of that stuff go away like it would stand still for a little while

The inability to think of alternative solutions when emotionally overwhelmed was also described by Sophia:

Sophia: I guess it feels quite a desperate situation even though in reality it's not...um and you know $\mathrm{i}$ - if I could be objective would be able to see other options... at the time it feels really really like it just feels like the emotion and the frustration or the stress whatever it is is just gonna just keep getting bigger and bigger and bigger and bigger and just it feels like I'll self-destruct um and I feel like I need to stop that and um cutting although it doesn't make me feel better um like its it it does s- stop that sense of it getting bigger and bigger and bigger...um but maybe it feels like I have had a bit of control of it

Even Ella who was able to weigh up alternative solutions only considered harmful options, such as taking an overdose or becoming intoxicated, which potentially would have served the same escape/avoidant function as self-injury.

\subsubsection{Discriminative Stimuli}

One of the reasons why people might have experienced constricted cognition is because of their verbal rules about NSSI. Verbal rules are SD's that specify a particular relationship between a behaviour and consequence (Farmer \& Latner, 2007; Miltenberger, 2004; Sturmey, Ward-Horner, Marroquin, \& Doran, 2007c). The rule becomes a SD because its presence makes it more likely that a certain behaviour will occur; that is, the verbal rule allows a person to discriminate when there is opportunity for reinforcement (Sturmey et al., 2007c). An example of a verbal rule is: If I cut myself, I will feel better. The rule serves as a signal to the person that relief (i.e., a negatively reinforced consequence) is more likely to be available if the person self-injures than if they do not self-injure. Several people provided examples of these rules in their descriptions of their most recent episode of NSSI, although the rules were rarely stated in cause and effect terms (i.e., if I do this, then this will happen).

Instead, I typically extrapolated from what people had said to identify rules that, in all likelihood, served as Sn's for their self-injury. People often talked about deciding to self-injure because they knew it would help. Ella commented that she 
had let the situation build up to a point where she "needed to do something quite drastic" to calm herself down. To her, cutting seemed to be the most appropriate action at the time and it is likely that the thought that cutting would help her calm down signalled an opportunity, as it had in the past, for relief following the behaviour. Similarly, cutting seemed "like the best option" to Sophia because, as she stated, when she had used it in the past it had worked to help her release negative emotions.

Four participants, however, provided more explicit examples of verbal rules:

Nicola: I think it was just like even just a day that I was just feeling like real...kind of empty...and...I was just like ah uh uh a- that'll help uh ((laughing))

Nora: it just progressed and then I got to the stage when I knew that it was the only way I was gonna make myself feel better was to cut myself so I did

Tara: the idea just came of...of course that's what I'll do to make myself feel better

Melanie: um I guess over the years I've sort of developed it as a means to cope when I do get like that...(mean) to settle myself down um...so yeah ((laughs)) I wanted to settle myself down ((laughing))

These extracts can all be interpreted as versions of the same verbal rule: If I want to feel better, then I need to hurt myself. These SD's would have worked in conjunction with the EOs in that the participants were motivated to gain relief from their overwhelming emotions and thoughts, and the verbal rules signalled to them that this relief was accessible through NSSI, as it had been in the past.

A more detailed example of how particular SD's and EOs interact to influence behaviour is evident in the effect that Emily's inability to sleep had on her. In the past, she had found sleep to be "a real escape" but as she began recovering from depression, she found it more and more difficult to get to sleep:

Emily: I think when when when you feel that releafs release and relief from from cutting usually what I'd find is that it would be uh so euphoric that it would tire me out...I found that that as I was getting healthier and as I was getting recovering from being quite so depressed...um if I was tired and stressed out and wanted to sleep I wasn't I wasn't gable to do it so easily

Emily's desire for sleep was one of the motivating factors behind her episode of selfinjury: 
Emily: it was still one of those things of those moments of getting stressed out and um feeling really down and and and fragile um where it was kind of like you know I kind of get to this point and I know that I was gonna do that...you know and that doing that would mean that I could go to sleep afterwards

In Emily's case the verbal rule appeared to be: If I cut myself, then I will be able to sleep. As such, the verbal rule (SD) and difficulty getting to sleep (EO) interacted to precipitate Emily's self-injurious behaviour.

Other SD's mentioned by participants were being alone; having access to implements to injure themselves with, or, as Angela phrased it, a "weapon of choice"; and the time of day. Tara talked about how once the thought that self-injury would make her feel better had popped into her head the search for something to hurt herself with became all consuming:

Tara: the idea just came of...of course that's what I'll do to make myself feel better and I think even as soon as that comes in I actually forget that I'm frustrated and it's just like that's the goal is of the feeling...is to then go and do that and so I then have to find a way it's like this little weird mission of my brain's that I'm on ((laughing)) that I've gotta find a way to then do that

For her most recent episode of NSSI, Tara found a piece of glass which she then used to cut herself:

Tara: I can't trust myself when I get stressed out to not break the really expensive razor open and do something so at the time I didn't have anything like that around and I think I found a piece of glass or something like that... and used that

Although the $S_{D}$ (i.e., the razor), which had been present in the past when Tara had self-injured, was not available, she had also previously used glass to cut herself when she did not have access to other implements:

Robyn: yuh so do you remember that time where you found the glass like the (unclear) Tara: yeah I think I was actively looking for it 'cos I think I'd actually found I'd done that before when I had...no other options

Pieces of glass had thus acquired discriminative status. Tara acknowledged that despite having practised other coping strategies, she became fixated on cutting as the solution, another example of constricted cognition prior to self-injuring:

Tara: $\quad$ I can look at it and go I could've stopped myself from even going and looking for that but I just kind of was so set on the idea that once I'd done that I'd be o- like that I could then get over it and be fine...I didn't really want to do it either because I'd been doing well at 
trying to...I guess use other ways to cope and I was actively like practising those but I guess this time it was just ((laughs))...was a bit much...um and that was for some reason why I decided ((laughing)) that this time I needed to for that reason

The power of specific SD's to influence behaviour was particularly highlighted by two participants. Karen described how she was unpacking her belongings after moving back to Wellington when she found a "little box of cutty things" which, in the context of her feeling depressed and overwhelmed, signalled the opportunity for relief following self-injury:

Karen: I found this little box that I've kept a lot of like razor blades and glass and things in and automatically it triggered me wanting to cut so um yeah I sat down on my bed cranked my music right up and I just started to cut

When I asked her if she still had the box, she replied:

Karen: $\quad$ yeah I do I don't know why I keep it um it's almost like a memento of times past ....and I mean I wouldn't use half the things in there ((laugh))...'cos half of them are like gross and old ((laughs))...um but yeah that reminds me of it and I don't have the heart to throw it away

Jenny had a very different attitude to one of the objects that she had repeatedly used to injure herself. After her most recent episode where she had stapled herself in the hand, Jenny had made the decision not to use staplers at all anymore:

Jenny: I made an informed decision after that that I'm not going to use that particular tool anymore...I call it a tool 'cos it's been it's become such a threat to me...I don't go near it at all...just just to be sure that I don't do anything you know

Sophia also reported trying to restrict her access to implements that she could use to hurt herself. The night before her most recent episode of NSSI, she had cut herself and had put the razor blades that she had used in a safe that only her husband could open. The next night when she wanted to injure herself again, she did not have access to the blades. However, she did have a small glass with her which she broke and then used to cut herself. It was not clear whether she had done this before. She had gone to bed early and being alone at the time may also have signalled to her that reinforcement would be available following NSSI. 
Certainly, being alone functioned as an SD for Paula's most recent episode as she was able to buy razor blades and go to the beach where she cut herself. Usually, she was carefully monitored by her caregiver:

Paula: the opportunity I got was when um my caregiver was at work 'cos she's quite the Nazi woman ((laughing)) [[Robyn laugh]] so she ((laughing)) she keeps an eye on me and she she's usually really good on picking up on um when I'm out of control as such and I don't have to say anything she can just tell...but yeah she was at work and so I saw I just took that opportunity and just I went on a walk and all of sudden the voice ${ }^{21}$ was just so much stronger and I was just so aching to feel that I went and brought blades and...I just sat at the beach and I tried everything to cry because I felt like I just needed to like all the pain I was holding was like I n- I needed like I I thought I should've been just sitting there exploding in tears and...I just couldn't there was just nothing nothing was happening and so I just sat there and I just kept dragging it across my like my wrist and my arm and then I just yeah just let rip and did it really quite badly really deeply and stuff

It is clear that particular $S_{D}$ 's (e.g., being alone, blades) allowed participants to discriminate when reinforcement was likely following self-injury, but that this reinforcement was only desired because of the EOs (e.g., feeling numb). Nicola encapsulated this interaction between the SD of a craft knife and the EO of feeling down in a matter-of-fact way, when describing the lead up to her most recent episode:

Nicola: I just broke up with my ex like...back then and it's like always emotional you know like ((laughing)) but um yeah and so I was down a l- ((laughing)) and I had a craft knife ((laughing))... and its I cut my leg

For Nicola, having access to a craft knife while feeling down led her to cut her leg multiple times, a behaviour that functioned as an escape from her negative intrapersonal experiences because it was followed by a sense of relief.

\subsubsection{Consequences}

All of the 18 participants whose episodes of NSSI functioned as escape or avoidance from negative intrapersonal experiences reported a reduction in, or the elimination of, negative emotions and/or thoughts during or following their selfinjurious behaviour. After cutting herself, Tara described feeling almost as though she had "a whole new start to the day as if the rest of the day hadn't gone so bad

\footnotetext{
${ }^{21}$ The voice that Paula is referring to is her negative mindset which she called her "Anorexic voice".
} 
and...nothing else had really happened". The most common words used by participants to depict these negatively reinforced consequences of NSSI were "relief" and "release":

Natalie: I guess it's sort of like a relief a it kind of lifts my spirits a little bit

Paula: just a massive amount of release

Nora: um I think I was feeling relief

Ella: I think um you know because of adrenalins and endorphins and stuff that you do get some sense of release from it

Emily: I think it was the usual kind of just relieved and tired

Maria: there is kind of a sense of relief I guess

For Josh, the sense of relief that followed cutting was analogous to taking off a really tight hat:

Josh: $\quad$ then when I do cut it's kind of uh a release it's like it's as strange it might be ((laughing)) to describe it it's like wearing a really really tight hat and then...taking it off and just going like ah...sweet you know sort of ((laughing)) yeah sub- it it kind of feels like that...um sort of yeah as a emotionally and it can feel like that physically as well ((laughing))

In their descriptions of the consequences of self-injury, participants

articulated several, possible underlying mechanisms of self-injurious behaviours. Some people reported that self-injury distracted them from aversive internal stimulation, thus providing respite from their intense emotions and thoughts. For instance, Nicola talked about how focusing on the act of cutting, to ensure she did not hurt herself too severely, was distracting:

Nicola: I guess it's just like takes my mind off everything 'cos its like...I don't wanna like hurt myself like really badly or anything...it's like kind of like I guess your mind is on something q- quite intently you know... whereas and if if you're finding it if like if I'm finding it really hard to focus on everything you know... and then I finally find something that I can like focus on

Lucy similarly described being distracted from her thoughts and feelings while cutting:

Robyn: $\quad \mathrm{mm}$ so in terms of um keeping on those sort of same lines about talking about the feelings and thoughts when you had finished cutting...can you describe whether those thoughts and feelings say that led up to it change 
Lucy: not really they always end up coming back but just like for a while it gives me something else to focus on

The degree of respite experienced by Lucy depended on the severity of her selfinjury and how much pain she experienced:

Robyn: so how quickly do they usually come back

Lucy: $\quad$ depends how bad I've cut really ((laughing)) like...if it's not bad they come back pretty quickly but if it's bad sometimes it kind of take a couple of hours...'cos I'm sort of focussing on stopping myself ((laughing)) bleeding and focussing on the pain

While Luke also commented on the interrelationship between the severity of his cutting and the relief he experienced, he additionally described how for him the letting of blood was a cleansing ritual:

Luke: I cut good and proper like...because I knew that I would feel relief and I did like it's almost from the moment you hit a point it may be one cut or four or five and usually for me it's about five... when there's enough blood coming and and I can feel the sting of the razor and that it's just like a it washes everything away...when nothing matters right at that time nothing...matters at all it even sort of afterwards and as I'm healing

Like Luke, Josh also cut himself multiple times to achieve a release. He described how one of his techniques was to cut in the same place over and over as he found that the continuous pain provided him with a more powerful distraction. For his most recent episode, he had cut himself in three different locations, but had also cut seven to eight times in each of those sites. For these participants, distraction from emotional pain was achieved through the physical experiences of pain, witnessing blood, and wound-care.

Other participants specified that they felt emotionally numb during cutting, which provided them with relief from the chaos of their overwhelming thoughts and feelings:

Karen: $\quad$ I was just feeling just numb and it was so much better than feeling ((laughing)) overwhelmed....and it was just yeah it was like a release just to feel numb just to get the headache out of my head kind of thing ((laughing))

Robyn: mm yeah so when you were $\mathrm{f}-$ you say you were feeling numb was that while you were actually cutting you felt numb

Karen: yeah it was....and it was like I was able to think more rationally and everyth-all the problems seemed to go away

Robyn: ah ok so is that during the cutting or afterwards

Karen: $\quad$ yuh that was during...it made me think what am I so stressed about you know ((laughing))...all these things can be fixed 
Robyn: $\quad$ mhmm so you kinda you said that you go numb...in what ways

Nora: um I think ((sighs)) ((laugh)) it's weird 'cos I kind of find with cutting myself it numbs me but it kind of wakes me up as well...like it works in two different ways and yeah I think just the feelings that I got when I cut myself would enable me to just it just feels like nothing else matters and my head that was all cloudy and stuff...is just silenced and all the thoughts aren't there for like a few moments...um yeah and it just numbs all the feelings that I had inside to one level that I don't have to deal with at that time...um it just puts them into one thing...so I can not think about them

For both Karen and Nora, the relief of having a break from their thoughts seemed to indirectly enable problem-solving. Cutting appeared to help Karen put her problems in perspective and as she said, "all the problems seemed to go away". In comparison, Nora still experienced similar thoughts following her episode of NSSI, but felt she was more able to deal with them because she was no longer overwhelmed by her emotions:

Robyn: did you find that um your thoughts changed [[Nora coughs]] like once you had cut yourself

Nora: $\quad$ um not really ((laughing))

Robyn: not really

Nora: no they were kind of yeah they were kind of still there...um definitely still there but I guess I felt like I could deal with them in a little bit more depth...um after I had cut myself...um it kind of I felt like it enabled me to get more of a clear headspace in terms of dealing with that and um not feeling so overwhelmed by everything that I was feeling at the time...yuh and just get clarity and try and pull out what emotion I was feeling and then be able to deal with that instead of it all being one big thing... and feeling like I couldn't deal with it 'cos it was so big and so hard um so yeah the thoughts were still there but I didn't feel as if they were so big

Robyn: ok...so it sounds like the thoughts stay stayed pretty much the same but the just the emotions become more manageable

Nora: mm yuh yuh um yeah and that enables me to kind of think about the thoughts more clearly and um what I can do what I could do to try and make them easier or better

Melanie described a similar experience of being able to problem-solve once she was feeling less emotionally aroused and her thoughts had stopped racing. While she was cutting, she began to feel a bit calmer, and more settled and in control of what was going on. Following her episode of self-injury, she reported being able to think more clearly, and, although the negative thoughts of self-blame and inadequacy were still present, she did not believe them as much:

Melanie: um and but you know I was able to think more clearly... about everything so you know I rang my boyfriend up and got him to come back so we could talk and sort things out like I was able to think...so it felt better...after ((laughing)) 
While the majority of participants reported feeling distracted during or after injuring themselves, two people described being distracted from their thoughts and emotions following their decision to cut and before they had actually injured themselves. As detailed earlier in this chapter, Tara acknowledged that once she had thought of self-injury as the solution, she became so focused on finding something to injure herself with that she forgot about how intensely frustrated she had been feeling. Similarly, making the decision to cut impacted on Lucy's mood:

Robyn: so what happens to those thoughts and feelings once you decide that you're going to cut Lucy: $\quad$ it differs sometimes they go away sometimes they don't sometimes they get stronger and then kind of affects how bad my cutting is if they go away and I just kind of stop feeling it's not as bad

Robyn: so this episode last night...did they go away or did they get worse Lucy: $\quad$ no they went away

In the past when the thoughts had remained present, she acknowledged that she needed to force herself to stop cutting.

\subsubsection{Summary}

The majority of the self-injury episodes analysed appeared to serve an escape/avoidant function. Intense, negative emotions and thoughts, associated with both distal and recent events, established the motivation for self-injury, while specific stimuli (e.g., being alone, implements) signalled an opportunity for relief or release following self-injury. Participants' ability to problem-solve and think of alternative solutions to their distress often became constricted, and verbal rules about needing to self-injure in order to gain relief predominated.

\subsubsection{Interpersonal Escape/Avoidance}

None of the participants' most recent episodes of NSSI appeared to function as a way to avoid, or escape from, interpersonal obligations or responsibilities. However, two participants did acknowledge that they had been motivated to selfinjure in the past for this purpose:

Karen: that's another way of self-harm for me is that I can actually make my potassium that low if I want to but um and I've done that in the past year I've just been so...miserable and I've wanted to hurt myself so I've done that I haven't done it deliberately to want to kill myself...I've done it make myself sick so I go to hospital...just to get myself out of situations ((laugh)) 
Sophia: I have taken medications at other times and um taken too much medication...um and that's often seems like quite a easy option because um one it it's accessible and $\mathrm{m}$ - most of the medications that I have are s- sleep-inducing...um so it really is an escape in that sense... and it means that I'm also kinda out of play so if I'm feeling like I can't cope with things that's kind of a legitimate question-mark legitimate but le- uh uh in my head it can feel like that's a legiti- you know I l- like I am like being actually physically sick...'cos if I you know if I take a whole lot of um drugs that I'm not meant to take in that amount then it has a real physical effect on me... and I can't actually you know like if if I'm awake I can't actually function that well so I can't be responsible for the kids so you know it kind of has that a follow-on effect

Overdosing on medication as a legitimate form of escape had resulted in Sophia being hospitalised on numerous occasions. She described being in hospital as "a good break":

Sophia: often just want to be um in hospital for a couple of days or you know something like that where the expectations like my own and what I think other people have on me I've got a it seems to then give a yeah give a legitimate reason why they they can't be met...

Robyn: so have you been to hospital a few times...(from) medication and taking too much medication

Sophia: yuh yuh...yuh over the years it's been quite a few times and um and although you know it's never a very pleasant experience um it does mean that things stop ...you know it does it does interject in your life and um uh you know often you're constrained by what the medical staff you know what whether you know like like where they i- they'll discharge you or not and so...there you know there's those things that you kinda have to just go along with um....which are quite good ((laughing)) um yuh yeah

Robyn: kind of removes that pressure of decision-making

Sophia: yuh yuh...yuh it takes the responsibility away

Although both Karen and Sophia had purposefully hurt themselves in the past to escape or avoid interpersonal demands, they had self-injured using types of self-harm that would result in hospitalisation and which are beyond the scope of this thesis. However, it is worthwhile noting that they felt they needed to resort to hospitalisation to gain sufficient respite and, in Sophia's case, to a type of self-harm that would render her physically incapable of carrying out her responsibilities as a wife and mother.

\subsubsection{Intrapersonal access}

Intrapersonal, positively reinforcing consequences (e.g., an adrenalin rush) were described by eight people following their episodes of NSSI, leading me to hypothesise that these episodes functioned as a form of intrapersonal access. It is important to emphasise that only one of these eight episodes functioned exclusively 
as intrapersonal access; most of the episodes in this functional category also featured in the intrapersonal escape category.

\subsubsection{Establishing operations and discriminative stimuli}

The types of EOs reported by Belinda, the one participant whose self-injury episode functioned exclusively as intrapersonal access, were comparable to those reported by participants whose episodes of NSSI functioned as forms of intrapersonal escape. Although Belinda had an extensive history of NSSI - she reported that at one stage she was self-injuring up to thirty times a day by cutting or hitting her head against hard surfaces - she conceptualised her most recent episode of NSSI as a relapse. In the months preceding her episode of NSSI, she had experienced several miscarriages, which had served as distal EOs for her self-injury:

Belinda: what led up to it was I was trying to have a baby and I had had two or no three miscarriages in a period of um like an- nine months...and they couldn't find any reason why and I was really stressed... and I felt really it was just something for me to blame myself for I thought ah it must be me I'm I must...just be really stuffed up and wrong and um I think yeah I was internalising a lot of a lot of blame...f- for whatever was happening

Belinda went on to describe more proximal EOs that motivated her to injure herself. She was preparing a salad for Christmas lunch when she burnt the sunflower seeds:

Belinda: suddenly it just kind of all compac- compounded on me and I was going ah you're so stupid you know you're such an idiot and you know now you've burnt the sunflower seeds which is like a small thing but it was just like the last straw... and I just found myself on the floor of the kitchen basically smashing my head against the um kitchen floor... and it was just like really it was quite sudden ((laugh))...then I was was like ah it really hurt

Apart from Belinda, all of the participants whose most recent episode was hypothesised to function as intrapersonal access endorsed experiencing relief or release from aversive intrapersonal experiences following their self-injury. The same EOs that I discussed in the intrapersonal escape section thus established the conditions for NSSI to function as an adrenalin rush. That is, participants were motivated to engage in self-injury while experiencing negative emotions and thoughts because past self-injury had been followed by desired intrapersonal consequences. Similarly, the objects or occasions that functioned as SD's (e.g., being alone, implements) for these particular participants, described in section 4.3.1.2, 
would have signalled to them the opportunity for positive reinforcement following self-injury.

\subsubsection{Consequences}

In the context of Belinda's learning history, I hypothesised that the pain of smashing her head on the kitchen floor was a positively reinforced consequence. When discussing her self-injurious behaviour, Belinda repeatedly described using self-injury as a way to punish herself:

Belinda: when I have self-harmed it's like yeah good you deserved that you...deserved this punishment and I really think that it's been quite closely linked for me to um well right back to to being a kid but probably ((clears throat)) early adolescence... where um I had some pretty full-on experiences around abusive relationships an- and being told I was really useful and hopeless and crap...I kind of started you know internalising a lot of messages that I heard...um and repeating them and kind of becoming my own worst enemy and self-harm was a way of actually feeling that I had been sufficiently punished for being innately bad...or useless or stupid or...fat or whatever the hell it was that I I was deciding to pick on myself for at that time

Belinda explained how the severity of her self-injury depended on how intense her negative thoughts were at the time:

Belinda: the words are screaming in my head...um different phrases like um you're st- you know you're fat and ugly you're stupid you're an idiot so it's a whole lot of words that actually screaming in my head while I'm doing it...so I'm not even really concentrating on what I'm doing....actually there's just words screaming in my head telling me this and the louder they scream the more I would they're not external they're in my own head...but um the more I would the deeper I would cut or the harder I would hit my head

Eventually, the cutting or hitting would alleviate her distress:

Belinda: there's a point where endorphin pain endorphin kicks in... and um you actually are at yeah you feel ok sufficiently punished...so you're like ok well yeah it's like this this this feeling of release...so if its yeah it's this big huge tension release a bit of a endorphin high...you feel relaxed

Belinda thus appeared to experience release through pain, which she stated "feels kind of good as well as painful".

Given that Belinda began hitting her head on the kitchen floor in the context of experiencing thoughts about being stupid and an idiot, the pain she then experienced, in all likelihood, would have positively reinforced her self-injury as it had in the past when she punished herself. Although she did not state that she 
experienced release following her most recent episode, this may have been because her self-injury was interrupted by her husband who came running into the kitchen. Historically, she had only experienced release when she felt sufficiently punished for her perceived failings. If she had stated that she had experienced tension release, her episode of self-injury would have then functioned as both a form of intrapersonal escape and access.

Two other participants also discussed pain as a positive consequence following their most recent episode of self-injury. As I discussed in the previous section on intrapersonal escape, Owen burned himself most recently in the context of self-blame, self-directed anger, and the fear of becoming psychotic again. Self-injury provided Owen with a sense of release from his negative emotions and thoughts, a release he seemed to attribute to the pain he experienced while burning himself:

Owen: $\quad$ yeah I was actually feeling quite quite good ((laugh))...I was feeling a bit of release.... a bit um ((sigh)) yeah it just it felt right when it's when it was when I was doing it...and so I kept on doing it and it felt it felt like um it felt like it was justified... as well like you know this was this is what I want this is what I wanna feel...it's just what you wanna feel...you just wanna feel that pain

In the context of his intense negative emotions, the pain felt good because Owen was motivated to punish himself. He noted how he would have reacted differently to the pain, had he not been experiencing self-hatred and self-blame:

Owen: it's weird because I couldn't do it now...like if I tried to do it now I couldn't hold it there that long...but when I'm feeling like that hatred or that intensity of emotion it's feels good...it feels I can hold it there

The EOs he experienced therefore had a direct impact on the value of the pain and because the consequence of pain was positively reinforcing, he was able to burn himself for longer.

Self-punishment was also a recurring theme in my interview with Tara. She described sometimes using self-injury to teach herself a lesson, and that her selfinjury would increase in severity over time as punishment for not learning her lesson from past self-injury. When I asked what kind of lesson she was trying to teach herself, she replied: 
Tara: $\quad h m m$ to be more like in control and more perfect and like ((laughing)) perfect's a really bad word to use but I do realise that I quite often think that I should be perfect...um be yeah more in control of and better at things than perhaps I am... which is kind of an issue that I know I have ((laughs)) is trying to be really great at everything

In regards to her most recent episode of self-injury, she acknowledged that pain was an important part of learning her lesson:

Tara: $\quad$ straight afterwards I kind of was just sort of checking to see a couple of minutes afterwards it's like that's when I it does start to hurt and so I was like well is that gonna hurt yeah ok and I kind... of almost feel like I need that afterwards just to remind myself I guess still part of that teaching myself a lesson thing

For Tara, it is likely that pain served as a positively reinforced consequence because it was evidence for her that she had punished herself sufficiently.

Apart from pain, other intrapersonal positive reinforcers identified by participants as having occurred during, or following, their episode of self-injury, were experiencing an adrenalin rush, feeling alive, or sublime:

Angela: you just feel a bit more alive when you're doing something like that

Nicola: uh it's really bad I I quite I enjoy it like it...it is like it's weird but it's like kind of almost fun ((laugh))...I guess I probably feel like better A for adrenalin and what not

Josh: (unclear) it's sort of sublime

Matt: $\quad$ I know how to get myself in into sort of the right state of mind where I can do it quite calmly...and just get the adrenalin rush... and then I burn myself and then I'm only focussing on that nothing else I'm just focussing on that pain and so I feel kind of calm and kind of high

Maria: it felt quite exhilarating like...I mean I I think you know all the adrenalin and everything....as well you physically feel quite hyped up but then afterwards I'd feel really exhausted it was like but during it it $\mathrm{w}$ - uh um yeah it felt very exhilarating and yeah I just felt very I'spose alive

Based on these descriptions, it is likely that these episodes of self-injurious behaviour would have been reinforced by the positive, internal stimulation experienced by participants.

\subsubsection{Summary}

For a number of participants, positive reinforcers, such as experiencing an adrenalin rush following self-injury, appeared to go hand-in-hand with negative reinforcers, such as experiencing a sense of relief. In these cases, it seems that 
negative emotions dissipated to be replaced with positive emotions. Pain was also identified as positively reinforcing for those participants who were motivated to punish themselves through self-injury.

\subsubsection{Interpersonal access}

Several participants identified that they had accessed attention, care, and support from other people following their most recent episode of NSSI; however, only two participants mentioned that they had been motivated to seek support prior to hurting themselves. Debra, who was the only person whose episode of self-injury appeared to function exclusively as a form of interpersonal access, had been visiting her ex-boyfriend when she became really upset. She left his apartment because she did not want to "make a scene" in front of his flatmate and sat around the corner of his building where she then hit her head repeatedly on the concrete in front of her.

\subsubsection{Establishing Operations}

The EOs that Debra described were comparable to those identified by other participants whose NSSI episodes had fulfilled intrapersonal escape and access functions:

Debra: $\quad$ I just I got really kind of upset I was with him in his room and I was just you know I was sort of thinking about what would be to come in the break-up like... and I knew how hard my previous break-ups had been so I was just thinking about how sort of difficult I was expecting it to be and I just got really upset

When I asked Debra what else she was thinking, she noted her inability to problemsolve when she is feeling upset:

Debra: when I'm upset sort of my ability to to rationalise things...sort of just goes and and um I dunno like things that are that are sort of seem like little problems just suddenly seem really big and so I I dunno I would guess I'd say I wasn't really thinking very straight... and I just I wasn't thinking about what I was doing and I just yeah I was just thinking about how bad things were

Although she struggled to remember exactly what she had been thinking, she guessed that it would have been:

Debra: just everything is hopeless and I've just fucked everything up and like yeah I sort of felt like er uh he'd broken up with me because he wanted to be single... and so he said you know this is nothing to do with you...but I just sort of felt like I'd screwed up everything and I had no idea what to do about it and and that I was just that I was just totally useless 
Self-blame and thoughts of being useless, which were antecedents commonly identified by other participants, thus also preceded Debra's episode of NSSI.

However, one of the differences about the episode Debra described was that she was motivated to self-injure in order to access support from her ex-boyfriend:

Debra: I was just you know I was really upset that he didn't wanna be together anymore and....and I dunno I mean I guess I sort of wanted to to show him how upset I was maybe but I also sort of just felt like like I didn't really have any options I mean I mean he'd broken up with me I couldn't you know it was sort of out of my control

Other participants described accessing support as a result of their self-injury, which may have reinforced their behaviour, but they did not report self-injuring in order to access support.

Another participant, whose self-injury was more likely to have been reinforced by the attention he received rather than by the concurrent negatively reinforced consequence he reported of feeling calm, was Matt. He was the only participant who had injured himself most recently in front of another person. His self-injury occurred in the context of feeling "deflated" because he had applied for two jobs and had been turned down for both:

Matt: I was really I was sort of annoyed I was like so the journalistic world doesn't want me and the government doesn't want me and I guess my reaction was well fuck it I may as well just keep doing what I do it's like this was gonna be excuse to straighten myself out to... act properly and I was like obviously I just don't fit into that world I may as well just keep being weird and I was just like fuck it I'll just burn myself again

When he declared to his flatmate and her friend that he was going to burn himself, the flatmate's friend requested his permission to watch:

Matt: my flatmate was like ah I don't wanna watch and her friend was like was like wow cool can I watch and I was like yeah sure um and so I put my knives on and I did like like three lines across my arm

The phrase "I put my knives on" refers to what Matt called his "favourite way" of self-injuring; he would put two bread knives on a stove element to heat them up and then burn himself with the thin edge of the knives.

Matt was atypical in that he had regularly self-injured in front of other people; Debra was the only other participant who identified that she had 
occasionally hurt herself when others were present. Whether Matt self-injured alone or front of others depended on the types of EOs (e.g., frustration versus a desire to be the centre of attention) he was experiencing:

Matt: $\quad$ I was more likely to be on my own when I was cutting...that I would be sitting in my room I'd be kind of frustrated or in a bad mood and I would just pick up my craft knife and I'd try and cut myself whereas the burning is more likely when I'd been drinking and there were like other people around... and it was kind of a show-offy kinda thing

His assertion that NSSI could be a "show-offy kinda thing" was congruent with his declaration that when he was younger, he had viewed his self-injurious behaviour as a form of "performance art"; a belief that had manifested in him posting a self-injury video on YouTube:

Matt: $\quad$ I discovered YouTube and I thought it would be cool to like video myself doing it and putting it on the Internet and because I didn't like I had two dots on my arm and I didn't like them so I decided...to cover them over with a line erm and that was more just a performance thing I think

Subsequent to his video posting, he had engaged in discussions with people who had left comments about his video and he had also told people about his posting.

\subsubsection{Discriminative stimuli}

It was also evident from the discussion I had with Debra about her history of NSSI that she had developed the verbal rule that if she hurts herself, then people will understand how distressed she is. She believed that she was a fairly unstable person and because she got upset often, she asserted that people were unable to distinguish between her varying levels of distress:

Debra: I guess for other people it's kind of hard to tell the times I'm really really upset from the times I'm just quite upset or whatever... and so I dunno it's sort of a like a way of showing people how upset I am

This verbal rule, apparent prior to Debra's most recent episode of NSSI in her acknowledgment that she wanted to show her ex-boyfriend how upset and sad she was feeling, would have served as a cue to Debra that there was the opportunity for her to access support following self-injury.

Owen was the only other participant who explicitly reported that he had selfinjured most recently as a way of communicating his distress to others: 
Owen: I mean maybe maybe if the probably the least likely but a little bit of attention...um because I was voicing that I wasn't feeling very good and wasn't very well for five days before I did it... and that I thought no-one was listening and the here I am getting unwell again and all the rest of it um so it was a little bit of that...in this time when previously it was it was none of that it was more just hate

However, as acknowledged by Owen, support-seeking was not his primary motivation for self-injuring and therefore may not have reinforced his behaviour.

\subsubsection{Consequences}

After hitting her head, Debra texted her ex-boyfriend to ask him to meet her:

Debra: he came down and was hugging me making sure I was ok...he was there and that made me...feel better

It is likely that his comforting response was positively reinforcing. Later on, when her head was hurting, she told him what had happened:

Debra: I was like ah it was really stupid and yeah he was just like yeah I thought that you might have been doing that $\mathrm{mm}$ just sort of gave me a hug

Compared with other participants, Debra did not identify pain as a positive consequence. Instead, she commented that the headache, which follows her head hitting, reminds her that it is "a dumb thing to do".

Owen similarly received support following his episode of self-injury when he told his case manager what had happened. This consequence, however, was temporally distant from his self-injurious behaviour when compared with the other consequences (e.g., release of negative emotions) he experienced. Temporally distant consequences are less likely to act as reinforcers (Anderson, 2007) and, as such, it is more likely that the negatively reinforced, intrapersonal consequence of emotional release would have reinforced his behaviour, rather than positively reinforced, interpersonal consequence of accessing support.

In contrast, Paula identified experiencing a "massive amount of release" after cutting herself, but still felt dissociated, and as though her self-injury was not enough, until her caregiver came home and comforted her:

Paula: $\quad$ she realised what I'd done and stuff and she cleaned it up and all that and she did make it feel a lot better... and just um was comforting me for the rest of the afternoon and evening 
It is therefore more likely that her cutting functioned as a form of intrapersonal access rather than escape, even though the care and support she received from her caregiver was more temporally distant from the self-injury than the release she experienced.

Attention, rather than support, in all likelihood reinforced Matt's selfinjurious behaviour. It was evident that he had received substantial attention over the years for self-injuring, which had partially served to maintain his behaviour. Given that he had been feeling rejected and deflated prior to burning himself most recently, the attention from his flatmate's friend, who he asserted was really impressed by his ability to burn himself without flinching, most likely served as further positive, interpersonal reinforcement:

Matt: she was really impressed 'cos she was like wow you didn't even flinch that was amazing but then I was like but that was kinda lame because I was kinda gonna do it anyway but the fact that she thought this was cool and wanted to watch kind of... encouraged me it was like yeah I've got an audience now I'm like performing....and then like her reaction like yeah that was really cool you didn't even flinch I was just like yeah I'm tough

Other participants similarly received attention from family, friends, or clinicians following their self-injury but in most cases it was not clear whether this attention was a reinforcing consequence, especially since these participants had not explicitly identified feeling unsupported or neglected prior to their NSSI episode. For some of the episodes, attention from others seemed to occur by chance, such as when Belinda received comfort from her husband when he interrupted her banging her head on the kitchen floor. In these instances, the person inadvertently communicated their distress to others but the goal of the behaviour was not necessarily to access support. However, the support that was received may still have served as a positive reinforcer for future self-injurious behaviour.

A few participants actively sought out support by telling people what had happened (e.g., Owen who told his case manager about the episode) and the attention they received may have reinforced their behaviour. However, in some 
cases, the attention was deemed to be an aversive consequence when the people they confided in were judgemental and critical of their behaviour.

\subsubsection{Summary}

Although some participants were motivated to seek attention through selfinjuring, for the most part they appeared to want to communicate their need for support. Matt was the only participant who self-injured specifically for attention rather than support. In some cases, the temporal delay between the self-injurious behaviour and the support received by participants made it difficult to assess the likelihood that these behaviours functioned as forms of interpersonal access.

\subsubsection{Aversive Consequences}

As I mentioned earlier, the aversive consequences of clinical behaviour problems are usually delayed (Goldfried \& Sprafkin, 1976), which makes it difficult to ascertain whether they function as punishers. Despite this limitation, it is still useful to present the consequences that may have served as punishers for the participants in the current study because they provide insight into why people may stop self-injuring. Additionally, I will briefly review the aversive consequences experienced by the three participants who did not report any reinforcing consequences as it is likely that their self-injury was punished rather than reinforced.

The majority of the aversive consequences ${ }^{22}$ that followed participants' most recent episode of NSSI represented four themes: negative intrapersonal experiences, the wound and scarring, restricted clothing choices, and unhelpful reactions from others. Since my intention in this chapter was to focus on the consequences that potentially reinforced and maintained participants' self-injurious behaviours, I will only briefly discuss the consequences that may have punished the behaviour.

\subsubsection{Negative emotions and thoughts}

Many people identified experiencing negative emotions and/or thoughts following their episode of self-injury. These included disappointment, anger, and

\footnotetext{
${ }^{22}$ Four participants did not report any aversive consequences.
} 
guilt at having self-injured again:

Karen: I always feel really horrible afterwards like I feel like I've let everybody down ((laughs)) I feel like I've let myself down....and I think I should've I should've been better I should've waited I should've held on longer I should've done this I should've done that ((laughing)) ... why have I done this you know I'll start reflecting and I always feel really guilty...I was so pissed off at myself when I did it a month ago I was like dammit it's almost been a year you know ((laugh)) I've just gone....and done it again dammit

Natalie: it's more disappointment at myself...because I gave in...I guess...I get really angry at myself because...it's not something I enjoy doing ((laugh))

Owen: the consequences really are...the realisation that you've done it yourself...that's the stuff that's really hard to deal with because you're you're you're attacking the person that you should care the most about... and that's that's quite detrimental... also it made me think that I was going backwards in my recovery... which was quite damaging

Nora: I think the most of would've just been with myself and...um feeling bad that I'd done it again and um yeah 'cos I did wanna stop but I knew that it was all I had at that time...um yeah I think that was probably the biggest consequence was my own self

All of these participants judged themselves negatively for their perceived failure to resist self-injuring. Moreover, Karen stated that her episode of self-injury "kinda opened a can of worms" and, as a result, she believed that she would self-injure again before the year's end.

Aversive emotional consequences not only appeared to stem from participants being unable to meet their own expectations, but also from the inability to meet societal expectations. Tara, in particular, reported feeling ashamed that she had transgressed the social boundaries of what is considered normal, a transgression which then impacted on how she perceived herself:

Tara: $\quad$ I mean at the time it's kind of dealing with my feelings but then I have to afterwards deal with the shame that I've done something that's not really...considered normal to myself and admit that to other people and....admit that even though at that time I did have other options available to myself because I had been trying other ways of coping I had for some reason I didn't use that....and so it kind of in some ways it was kind of hard 'cos it reinforces my whole thoughts of I'm not normal I don't do things normally I should do things normally kind of... a little bit of a merry-go-round that I go on

In contrast, Ella was not ashamed of, or disappointed in, self-injuring, but rather was frustrated that it did not have the same effect that it used to:

Ella: I guess you kind of you you go back to your old coping strategies...or your old comforts um habitually and so I thought I think I thought that it would do more than what it did ...I think rationally um I kind of yeah I kind of thought well that was a waste of time um 
...'cos it it didn't yeah I mean I ((sighs)) it helped a little but uh I don't know maybe I'd talked myself into thinking it was gonna like magically...make me feel wonderful ...which of course it's not gonna do

A couple of the other participants similarly commented that their most recent episode of self-injury had failed to live up to their expectations, which in Josh's case led to him feeling as though he needed to self-injure again to get the desired effect: Josh: $\quad$ the sort of main I guess problem with last week was um having done drugs...as well as um self-harm so it's kind of um I didn't get as much of the sort of sublime...feeling um as I normally would've...I just more s- sort of had like a uh sort of awkward feeling really ((laughing))...normally I wouldn't actually continually think about you know um it afterwards I'd just kind of ((clears throat)) go with the fl- go with it basically... whereas um yeah with with the drugs I was still kind of aware of...the reason why I was doing that which kind of made me feel like I needed to do it again

Josh's expectations of how he would feel following cutting were unfulfilled because of the dampening effect of drugs, whereas Nora's expectations were unfulfilled because self-injuring did not solve her problems:

Nora: I felt quite bad because I knew that it hadn't solved anything like...this problems that were there before it were still there...um yeah kind of disillusioned that they hadn't gone ...um and that it hadn't just disappeared when I cut myself...that all the feelings hadn't just gone...out of me

\subsubsection{The wound and scarring}

Physical wounds and scarring were identified by participants as both shortand long-term negative, consequences of their most recent episode of self-injury. For some participants, the short-term consequences focused on the discomfort associated with the wound:

Ella: it's quite uncomfortable while y- you know you're healing and stuff

Debra: I guess yeah the times I hit my head my head is sore and I'm kind of just like well that was stupid this now I have a headache well that was a dumb thing to do

However for Luke, the location of the wounds was distressing because they were difficult to conceal:

Luke: $\quad$ and yeah so this time...it was kind of disappointment...like ah too too many too visual I I got really upset with myself because they were too close to my sleeve line 
The difficulties with having to conceal wounds were also associated with the aversive, long-term consequence of scarring. Angela considered scarring to be sufficiently adverse for it to deter her from engaging in NSSI more frequently:

Angela: afterwards I um I don't feel sad about doing it or anything like that because I think with depression well with mine anyway um there's that feeling that you're just you're worth nothing basically so it's not really that I feel sad about having cut myself but I k- I just worry about scarring and things like that...if that wasn't a worry in my mind I think I would do it more often

In contrast, scarring did not appear to be a deterrent for Karen but she did regret the permanence of her "marks":

Karen : I think what have I done to myself these marks are gonna be here forever 'cos I scar quite dark

Of course, the potential for scarring was not a protective factor against future selfinjury for all participants; Nicola mentioned that the scar from her most recent episode had "pretty much gone now" so she did not rate it as a negative consequence.

\subsubsection{Restricted clothing choices}

Another set of possibly punishing consequences revolved around how the location of the wounds and the extent of scarring restricted participants' clothing choices:

Karen: and then of course it's like ah no I'm gonna have to hide it now and gonna have to bandage it up and gonna have to wear long sleeves for however long... but of course if I've just cut ((laugh)) I'm not gonna walk around with a blatant bandage on my arm ((laughing)) 'cos that just makes it obvious and like...I don't want everyone to know so

Tara: $\quad$ and I guess also um another consequence of it is that I think it was well it was just coming up to winter so it wasn't so bad...but it means that I'd had to wear long sleeve tops for a long time

Luke: I can't wear a t-shirt for another three months...thank god it's winter you know like...like I mean they seem really sort of surface but that that becomes a big issue... when you're thinking about what you've done is ah god here we go...like no t-shirt again for three months like...that can be quite sad

Lucy: the main problem's just covering it up

It was difficult to determine exactly why people covered up; multiple reasons could have motivated participants to hide the evidence of their self-injury, including a 
desire to keep their behaviour a secret in order to retain control over it or to avoid being judged by others.

\subsubsection{Reactions from others}

Indeed, the anticipation of being negatively evaluated by others was one of the reasons Debra gave for not having cut herself more often:

Debra: the times I've cut myself I sort of its it left a scar that stayed for $\mathrm{f}$ - three or four months but now... its sort of gone... which I am pretty glad about 'cos I dunno I mean like I mean I'll tell my close friends about it but I don't want just random people... thinking I'm that kind of person....a couple of times when I've thought of cutting myself the fact that it would scar has been a factor in me not doing it because...I I mean people will really judge you about that kind of thing

When Debra anticipates being judged as "that kind of person", it is unclear what type of person she is referring to. Other participants, however, did report incidents where they were judged for their most recent episode of NSSI; judgements that were often at odds with how they perceived themselves.

Both Karen and Ella were frustrated with how they were evaluated as unstable and mentally unwell by medical and mental health professionals:

Karen: I was um in hospital um just after I'd done them like two weeks after I'd done them for something ah what did I go in for ah it was some kind of health check thing anyway um they the scars only just healed...so they were still a little bit new and um the guy was like I was like ah they're two weeks old man you know and he's no they're not and I'm like yes they are ((laugh)) no we need the CAT team now and it was like nah oo they ah for god's sake you know ((laughing)) so frustrating like no they're old I you know they've healed...so that kind of thing really frustrates me it's like ask me first and take my opinion seriously ((laughs))

Ella: when I said I actually feel like I'm ok and...you know I I did this yuh I I did this once and for me I feel like it was a slip-up and I feel like I've got past it and my psychiatrist went yeah but you've said that before and I was just like ah what the hel- like and it was almost like I was written off completely.... and I was back to being that you know really small little crazy mental patient in the corner um just like that over one decision I'd made... which I was ok with ((laughs))... and then they couldn't understand why I got angry ((laughing))

It was clear that being made to feel like "a small little crazy mental patient" or someone who cannot be trusted was an extremely disempowering experience for Ella and Karen, both of whom felt unfairly judged by clinicians. 
Owen was similarly judged solely on the basis of his self-injury when, after

his most recent episode, he was questioned as to whether he was a "burner":

Owen: my most recent episode I um burnt myself and then walking around and and everyone going what happened what happened... are you a burner are you wh- what's going on just all that stuff made it me think nah this is really you know this stuff really isn't what I wanna be about

Having his identity reduced to one particular behaviour choice may have had some punishing value for Owen as he cites this as a reason for stopping self-injury.

Conversely, when Melanie was judged negatively for cutting herself, her reaction was to justify her use of self-injury as a coping mechanism. She had texted her ex-boyfriend to tell him what had happened and he subsequently informed her mother that she had self-injured:

Melanie: ((laugh)) had a bit to deal with there when she came home ((laughing))...mm she doesn't really understand it at all so...she was very upset said she thought I had got past all that and...that it wasn't a good way to deal with things and ((laughing))...I sort of had to tell her yes I know that but ((laughing)) it's a way to deal with things ((laughing))

The manner in which Melanie dealt with this altercation makes it seem unlikely that her mother's reaction would have punished her self-injurious behaviour. What seems more likely is that her mother's reaction would have acted as a punisher for her contacting her ex-boyfriend following any future episodes of self-injury.

For Jenny, other people's reactions to her self-injury did not appear to concern her as much as God's reaction:

Jenny: the only consequence really after that last one was I was afraid of how God might see me...because I kept asking him to heal me and...but I kept doing what I did and I'd done it the last time I was thinking I wonder what he's thinking looking down at me thinking there you are I tried to make you better but y- what are you doing you're still going back to it... and I felt an immense sense of responsibility for that that he might perceive me as being a very um I dunno arrogant in some way uncooperative maybe...just taking advantage of him...that was almost enough as a consequence for me that he might you know send something down on me or...punish me or in some way or be very unhappy with me

What is particularly striking about Jenny's admission is her palpable sense of selfblame and belief that it was her fault for not being healed. 
Matt was the only person to experience an admiring reaction to his self-injury as a possibly punishing consequence. Self-injuring in front of his flatmate's friend resulted in him feeling lame and immature:

Matt: I felt immature...like that's probably the same thing as b-feeling lame I just felt like I was doing I was doing it because it's what I used to do and I had no other better reason and that eh and I felt kind of bad about the fact that I was like showing off to this person and not setting a good example...I guess the person who was watching me is a little bit unstable herself um she has her own way of dealing with things usually by getting drunk and you know I think the fact that she was like thought it was really interesting when I was doing it was like an extra reason it was like cool I have an audience...but I realised pretty much immediately afterwards that that wasn't necessarily good for her and so I didn't feel particularly guilty about it but again...I was just like ah that's a bit silly that's a bit stupid of me

Matt's realisation that he self-injured more out of habit than need and that he has a responsibility to set an example to those younger than him, may deter him from harming himself in similar situations in the future.

\subsubsection{Exclusive experience of punishing consequences}

As I mentioned earlier, three participants identified experiencing exclusively punishing consequences following their most recent episode of NSSI, which included feelings of shame, disappointment for giving in to the urge to self-injure, and concerns about the wound getting infected. These consequences were not substantively different from the punishing consequences reported by participants who had also experienced reinforcing consequences following their episode of NSSI.

\subsubsection{Summary}

The aversive consequences identified by the participants reflected the following four themes: negative emotions and thoughts, physical effects of the wound and concerns about scarring, needing to restrict their clothing choices to hide the evidence of their self-injury from others, and experiencing unhelpful and stigmatising reactions from others. As such, the majority of these consequences stemmed from the impact of their self-injury on the way in which they viewed themselves, how they anticipated being perceived by others, and, in some cases, the way in which they actually were judged by others. 


\subsubsection{Summary of Interpretative Functional Analysis results}

Overall, the most frequently identified function was intrapersonal escape/avoidance, but many of the participants' self-injury episodes were hypothesised to serve more than one function. Interpersonal functions were far less common than intrapersonal functions, with only a few episodes hypothesised to function as a form of interpersonal access and none as interpersonal escape/avoidance. Many of the EOs and Sn's did not appear to differ according to the function of the behaviour, but rather were the same across functions. For example, negative emotions and thoughts commonly precipitated NSSI episodes that served intrapersonal or interpersonal functions, highlighting the importance of individual learning histories in maintaining self-injurious behaviours.

\section{STAGE THREE: EMAIL OR TELEPHONE FOLLOW-UP}

Approximately two days after each interview, I contacted the participants by email or phone to once again thank them for taking part in the study and to ask them whether they had thought of anything else that they wanted to share with me. This was recommended by one of the consumer advocates I consulted as a way of checking in with, rather than checking up on, participants. I also hoped that by initiating contact with participants after the interviews, they would be more likely to contact me if they had any concerns or questions about the study.

Some participants responded to my email enquiry by sending follow-up thoughts on diverse issues such as their reasons for self-injuring, other people's misconceptions and assumptions about NSSI, and the link between direct and nondirect forms of NSSI. One woman emailed me the diary entry she wrote the night of the interview noting that she did not realise how much more she wanted to say. In her diary entry, she reflected on why she cut (e.g., "Cutting allowed me to escape") and the effect that it had on her:

I let cutting become such a big part of me, and lost my emotions, and sense of who I was, and any control I had over that. My body felt numb and empty, but my head was full, and cloudy, and heavy. Kind of like the air when it's about to rain. Cutting enabled me to let some of the cloudyness go, and left me feel fresh, kind of like the air after it's rained. 
Other participants emailed to comment on their experience of taking part in the study:

Just glad to be a part of something that will help others.

I just wanted to say, thanks for the opportunity to tell my story. As nervous as I was, after the experience I felt very liberated and a bit less alien than before I started.

I didn't think of anything else yet, that's probably the most I've ever talked about self harm in one go anyway! I felt good afterwards though, I think it was a good opportunity to process some stuff and reflect on it for myself.

\section{STAGE FOUR: FEEDBACK QUESTIONNAIRE}

\subsection{Participants and procedure}

Approximately two weeks after being interviewed, participants were sent a questionnaire (see Appendix G) to provide anonymous feedback about their experiences of taking part in the study, which they were asked to complete and return within two weeks. Twenty-three people (95.8\%) completed their questionnaires; the one person who did not return their questionnaire later wrote to apologise that he had been away at the time. Unfortunately, I was unable to calculate the average number of days between the interview participation and questionnaire completion because the questionnaires were anonymous. Each person was posted a movie voucher for completing the questionnaire. ${ }^{23}$

\subsection{Measure}

As I was unable to locate a previously published questionnaire that related specifically to how people had responded to taking part in a study about self-injury, I adapted the Reactions to Research Participation Questionnaire for Parents (RRPQ-P; Kassam-Adams \& Newman, 2002, 2005) for the purposes of the current study. The RRPQ-R consists of 12 items rated on a 5-point scale from strongly disagree to strongly agree and has demonstrated acceptable internal consistency scores of .78 to .80 (Kassam-Adams \& Newman, 2002, 2005).

\footnotetext{
${ }^{23}$ One participant received a $\$ 10$ voucher for the Warehouse instead of a movie voucher because she had hearing difficulties.
} 
Nine of the 12 items in the RRPQ-P (Kassam-Adams \& Newman, 2005) were included in the research evaluation measure used in this study. Of these nine items, two were taken verbatim from the RRPQ-P, one was split into two items, and seven were modified (e.g., "I am sorry I was in this study" was rewritten to read, "I regret participating in this study"). A further seven questions were added to the measure (e.g., "The study was explained thoroughly to me before I took part").

This resulted in a total of 17 items in the revised measure; participants were asked to respond to the first 15 items on a 5-point likert scale from 1 (strongly disagree) to 5 (strongly agree). These 15 items focused on issues of informed consent, rapport, emotional reactions to the interview, and beliefs about study participation. The final two items were open-ended and asked whether participants had any suggestions about how to improve future research studies on NSSI and whether they had any other comments to add about their experiences of being in the study.

\subsection{Results}

The results of the research evaluation questionnaire are presented in Table 3. All of the participants agreed or strongly agreed that they had given informed consent to participate in the study and that they knew they could skip questions or take a break from the interview whenever they wanted to. Only one participant was not sure that they could stop being in the study at any time. The participants reported feeling comfortable and respected during the interview. People were more likely to endorse feeling sad or upset, rather than distressed, as a result of participating in the study. Almost half of the participants agreed that being in the study had made them feel good about themselves and all but one of the participants believed that their involvement in the study was helpful to other people who selfinjure. Despite some people reporting that they felt sad or upset because of their participation in the study, 22 people did not regret taking part and all 23 participants agreed or strongly agreed that with the benefit of hindsight and knowing what it was like to take part in the study, they would still choose to participate. 
Table 3

Number of participants $(N=23)$ who endorsed each statement

\begin{tabular}{|c|c|c|c|c|c|}
\hline Item & $\begin{array}{c}\text { Strongly disagree } \\
N(\%) \\
\end{array}$ & $\begin{array}{c}\text { Disagree } \\
N(\%)\end{array}$ & $\begin{array}{c}\text { Not sure } \\
N(\%)\end{array}$ & $\begin{array}{l}\text { Agree } \\
N(\%) \\
\end{array}$ & $\begin{array}{c}\text { Strongly agree } \\
N(\%) \\
\end{array}$ \\
\hline The study was explained thoroughly to me before I took part. & $0(0.0)$ & $0(0.0)$ & $0(0.0)$ & $2(8.7)$ & $21(91.3)$ \\
\hline $\begin{array}{l}\text { It was my choice to take part in the study (i.e., I could have said } \\
\text { no even if other people wanted me to say yes). }\end{array}$ & $0(0.0)$ & $0(0.0)$ & $0(0.0)$ & $1(4.3)$ & $22(95.7)$ \\
\hline I knew I could skip questions or parts of the study if I wanted to. & $0(0.0)$ & $0(0.0)$ & $0(0.0)$ & $4(17.4)$ & $19(82.6)$ \\
\hline I knew I could stop being in the study at any time. & $0(0.0)$ & $0(0.0)$ & $1(4.3)$ & $1(4.3)$ & $21(91.3)$ \\
\hline $\begin{array}{l}\text { During the interview, I knew that I could ask to take a break } \\
\text { whenever I wanted. }\end{array}$ & $0(0.0)$ & $0(0.0)$ & $0(0.0)$ & $1(4.3)$ & $22(95.7)$ \\
\hline The interviewer made me feel comfortable during the interview. & $0(0.0)$ & $0(0.0)$ & $0(0.0)$ & $1(4.3)$ & $22(95.7)$ \\
\hline $\begin{array}{l}\text { The interviewer showed respect for my feelings and experiences } \\
\text { during the interview. }\end{array}$ & $0(0.0)$ & $0(0.0)$ & $0(0.0)$ & $2(8.7)$ & $21(91.3)$ \\
\hline I am glad that I took part in this study. & $0(0.0)$ & $0(0.0)$ & $0(0.0)$ & $10(43.5)$ & $13(56.5)$ \\
\hline Being in this study made me feel distressed. & $4(17.4)$ & $13(56.5)$ & $6(26.1)$ & $0(0.0)$ & $0(0.0)$ \\
\hline Being in this study made me feel upset. & $3(13.0)$ & $14(60.9)$ & $3(13.0)$ & $2(8.7)$ & $1(4.3)$ \\
\hline Being in this study made me feel good about myself. & $0(0.0)$ & $3(13.0)$ & $9(39.1)$ & $10(43.5)$ & $1(4.3)$ \\
\hline I regret participating in this study. & $17(73.9)$ & $5(21.7)$ & $1(4.3)$ & $0(0.0)$ & $0(0.0)$ \\
\hline Being in this study made me feel sad. & $2(8.7)$ & $10(43.5)$ & $3(13.0)$ & $7(30.4)$ & $1(4.3)$ \\
\hline $\begin{array}{l}\text { I believe that by being in the study I am helping other people who } \\
\text { self-harm. }\end{array}$ & $0(0.0)$ & $0(0.0)$ & $1(4.3)$ & $12(52.2)$ & $10(43.5)$ \\
\hline $\begin{array}{l}\text { Knowing what I know now about participating in the study, I } \\
\text { would still choose to take part in this research. }\end{array}$ & $0(0.0)$ & $0(0.0)$ & $0(0.0)$ & $3(13.0)$ & $20(87.0)$ \\
\hline
\end{tabular}


Reasons as to why participants valued participating in the study can be

gleaned from their responses to the open-ended questions:

It was fantastic to partake in such an important study. Hopefully it will help ascertain and clarify what is going on in the minds of many young people who self harm, and what steps can be taken to actually support people who do. Self harm is a societal taboo in many ways, hopefully studies as these help to dispel the myths that even the medical profession sometimes adheres to still. We need to move away from the belief that asking people about their self harm is "dangerous" and will cause people to self harm more. There is no evidence to support these assumptions. Many people who self harm would probably rather people asked than made misguided assumptions.

I'm really glad I participated. I haven't had the opportunity to talk about these issues or process it for myself before. I felt ready to reflect on it and very safe and respected in this process. I hope the research is well received and helpful to others.

I found that I felt quite relieved after the study as I've never really spoken about my self harming. It was good to get it off my chest and I felt like I was helping other self harmers out there. I actually really liked being in this study. It felt really good to be able to talk freely and openly about self harm, in an environment that felt safe and non-judgemental...Although at times, I felt a little bit sad and distressed, it was only slightly, mostly because of taking myself back to my experiences with self-harm, but I liked the fact that I felt that somehow my experiences may eventually be able to help others...

I did not find taking part in this research to be at all distressing or triggering, and actually found it quite positive-helped me process.

It was easier to talk about self harm in a research participation situation because it was more about being able to possibly help someone else, which bypasses the natural instincts to avoid talking because of feelings of no or low self worth or self importance.

Taking part in the study thus appeared to be a positive experience for participants because they were able to discuss and reflect on their experiences of self-injury without feeling judged and, at the same time, they felt as though their experiences may help others in the future who are struggling with similar issues.

\section{DISCUSSION}

\subsection{Forms and frequencies of NSSI behaviours}

The majority of the people who participated in the current study had an extensive history of NSSI and, on average, had used eight different types of selfinjury in their lifetimes. The extent of NSSI in this sample was unsurprising given that several participants were recruited through mental health services and a mental health consumer network, and the prevalence of self-injury is substantially higher in 
psychiatric compared to community populations (Bebbington et al., 2010; Briere \& Gil, 1998; Claes, Klonsky, et al., 2010). A number of university students similarly reported high rates of NSSI in the current study, but most of these students were currently receiving mental health support, or at least, had received such support in the past.

Several participants reported types of self-injury that did not meet the definition of NSSI used in this thesis. Two examples of other forms of self-injury, in particular, warrant further discussion. To gain admission into hospital, Sophia acknowledged overdosing on medication several times; while Karen, who had been diagnosed with Bulimia, had ensured that her potassium levels were dangerously low (see section 4.3.2). Both of these participants were motivated to be hospitalised to escape from, or avoid, interpersonal responsibilities and, as such, it is likely that these episodes functioned as forms of interpersonal escape/avoidance.

These examples suggest that restricting the types of NSSI to exclude behaviours such as overdosing may have decreased the likelihood of observing certain functions. Indeed, none of the participants reported self-injuring most recently as a form of interpersonal escape/avoidance. Further research is necessary to determine whether particular types of NSSI are aligned with specific functions.

\subsection{Is self-injury primarily an experientially avoidant behaviour within Aotearoa}

\section{New Zealand?}

Functionally analysing participants' most recent episode of NSSI enabled me to identify considerable homogeneity among complex, seemingly heterogeneous incidents of self-injury. The majority of the self-injury episodes were hypothesised to fulfil an intrapersonal escape/avoidance function, which is synonymous with experiential avoidance. In contrast, no episodes were hypothesised to serve an interpersonal escape/avoidance function.

Access functions commonly occurred in conjunction with escape/avoidance functions. Only one episode functioned exclusively as a form of interpersonal access, while one other episode functioned exclusively as a form of intrapersonal access. For 
the latter episode, the participant was interrupted by her husband while she was hitting her head on the kitchen floor. The behaviour may have fulfilled an escape/avoidant function, as it had numerous times in the past, if she had not been interrupted.

While the findings from my Interpretative Functional Analysis are broadly consistent with the EAM (Chapman et al., 2006) in that the majority of episodes functioned as a form of escape from aversive thoughts and emotions, they also provide insight into the ways in which self-injurious behaviours function that are undervalued within, or excluded from, the contingencies depicted in the EAM (Chapman et al., 2006). These insights include the importance of cognitions and clarifying the role of positive reinforcement in maintaining NSSI.

\subsubsection{The role of cognitions}

Cognitions are not completely dismissed within the EAM, but Chapman and colleagues (2006) do maintain that self-injury is predominantly an emotionally avoidant behaviour. Additionally, they suggest that emotional avoidance may underlie attempts to escape from negative cognitions; in other words, it is the emotions connected to negative cognitions that motivate people to engage in avoidant behaviours. An explicit focus on the emotional precipitants of NSSI makes for a more parsimonious model, but has far-reaching research and clinical implications.

To date, researchers have largely neglected the role of cognitions in NSSI, preferring instead to concentrate on explaining how self-injury is used to regulate emotions (e.g., Adrian et al., 2011; Kamphuis et al., 2007). Models, such as the EAM (Chapman et al., 2006), may perpetuate this limited focus through deemphasising the influence of unwanted cognitions on self-injurious behaviours. In the current study, cognitions featured heavily in participants' accounts of their most recent episode of self-injury. More research is needed to decipher whether specific types of cognitions and/or particular cognitive processes commonly precipitate NSSI. 
During the interviews, one of the most frequently mentioned cognitive processes to occur prior to self-injury was that of constriction. Cognitive constriction is typically associated with suicidal behaviours (Leenaars, De Wilde, Wenckstern, \& Kral, 2001; O'Connor, Sheehy, \& O'Connor, 1999) and is defined by Schneidman (1996) as: "a tunneling or a focusing or narrowing of the range of options usually available to that individual's consciousness when the mind is not panicked into dichotomous (either-or) thinking" (p. 134). An example of constricted thinking in the context of a suicidal crisis is: "If this disease is incurable, I will end it all" (Walsh, 2006, p. 13).

This conditional, if-then formula is identical to that of the verbal rules discussed earlier in this chapter (see section 4.3.1.2), which suggests that cognitive constriction may be a key component of NSSI. Although Pattison and Kahan (1983) listed cognitive constriction as one of the symptoms of their deliberate self-harm syndrome, it has been largely overlooked in empirical investigations of NSSI. On the contrary, Walsh (2006) recommends differentiating between suicidal and nonsuicidal self-injurious behaviours on the basis of cognitive constriction.

According to Walsh (2006), people who engage in NSSI typically demonstrate disorganised, not dichotomous, thought patterns, and consider NSSI as one of a number of coping strategies they could use. Participants in the current study did indeed perceive that they had various options available to them but these perceptions usually occurred in hindsight, after they had engaged in NSSI. When discussing their thought processes prior to self-injuring, many participants reflected on how it seemed that NSSI was the only option they had at the time to effectively cope with distress. More research is needed on how people reach the point where their cognition becomes constricted. For participants in the current study, this process seemed to occur when they surpassed a certain threshold of emotional arousal, which then interfered with their ability to problem-solve alternative solutions. Decreasing their emotional arousal through self-injury then facilitated future problem-solving and provided them with a sense of agency. 
Understanding more about the role of verbal rules (as a form of cognitive constriction) in precipitating NSSI episodes thus has important clinical implications. As SD's, verbal rules signal to people when reinforcement is likely to be available following self-injury and therefore help to maintain self-injurious behaviours (Sturmey et al., 2007c). Clinicians therefore may need to explicitly identify and target verbal rules in treatment.

\subsubsection{What about positive reinforcement?}

Several self-injury episodes appeared to facilitate access to positive intrapersonal experiences or support, highlighting the role of positive reinforcement in the maintenance of self-injury. As discussed earlier in this thesis, a number of researchers have investigated whether NSSI is a positively reinforced behaviour (e.g., Lloyd-Richardson et al., 2007; Nock \& Prinstein, 2004) and results are consistent with the findings of this study, which suggest that positive reinforcement is important for some people.

However, it is notable that positive reinforcement schedules were seldom evident in the absence of negative reinforcement. This suggests that co-existing reinforcement schedules operate for some people who self-injure, making it potentially more difficult to extinguish the behaviour. These results are consistent with Brown et al.'s (2002) study where participants reported multiple reasons for single self-injury episodes. Certainly, Sturmey et al. (2007b) contend that focusing on single antecedents and consequences of operant behaviour is simplistic as concurrent reinforcement schedules are the norm.

Another important finding is that very few people engaged in self-injury to access attention, which is contrary to anecdotal perceptions of NSSI as an attentionseeking behaviour. More typically, when people did report receiving attention as a direct result of self-injuring, it was perceived to be an aversive consequence. Only two people acknowledged being motivated to engage in self-injury to gain attention from others, although it is possible that some participants may not have reported such motivations if they perceived them to be socially undesirable (Nock, 2008). 
Intrapersonal positive reinforcement, however, was more common. For example, experiencing pain in the context of a desire to punish oneself for perceived wrongdoings potentially functions to positively reinforce self-injurious behaviour.

\subsection{Strengths of Interpretative Functional Analysis}

The detailed functional assessments undertaken for this study provided valuable insights into specific antecedents (e.g., verbal rules) and consequences (e.g., attention) of self-injurious behaviours, which have been largely ignored, or at times misrepresented, in the literature. To my knowledge, no other researchers have distinguished between EOs and So's when examining the role of antecedents in precipitating self-injurious behaviours among typically developing populations, or conducted a group-level comparison of functional assessments of NSSI.

Furthermore, interviewing 24 people enabled me to access a wide range of selfinjury experiences.

\subsection{Limitations of Interpretative Functional Analysis}

Conducting idiographic assessments allowed me to hypothesise about which consequences may reinforce participants' self-injurious behaviours, but to qualify as a reinforcer these consequences would have had to increase the frequency of participants' self-injurious behaviour (Dougher \& Hayes, 2000). Since each participant was only interviewed once, I cannot confirm whether the hypothesised reinforcers actually did reinforce participants' self-injurious behaviour because I do not know whether they self-injured again.

Furthermore, several of the participants had self-injured months before the interview and the extent to which their accounts of their most recent episode were influenced by retrospective biases is unclear. The length of time between the episodes and interviews also precluded a more detailed behaviour chain analysis, similar to those conducted as part of DBT (Lynch et al., 2006).

However, chain analyses are likely inappropriate for one-off research participation given that people frequently experience these as arduous; it has even been suggested that they may function to punish NSSI (Lynch et al., 2006). If I had 
been able to elicit more detailed information from participants, it would have still been difficult to achieve absolute certainty about the functions of the behaviours due to the complexity of concurrent reinforcement schedules (Sturmey et al., 2007b) and the inability to manipulate specific variables.

Finally, many more women than men participated in the current study, which is common in much self-injury research (Russell et al., 2010). In light of recent studies that suggest there are no significant gender difference in the prevalence of NSSI (e.g., Andover et al., 2010; Claes, Houben, et al., 2010), it is unclear why so few men participated. One possibility is that males may have felt more uncomfortable disclosing stories about their self-injurious behaviour in a socio-cultural context where NSSI is still viewed as a largely female phenomenon.

On the surface, the process and functions of the self-injury episodes described by the male participants in this study did not appear different to those described by the female participants. Although the only participant to self-injure regularly in front of others was male, it is unknown whether self-injury as performance for others is a gendered phenomenon. One of the few studies to investigate whether self-injury functions differ according to gender found that men were significantly more likely to report sensation-seeking (Klonsky \& Glenn, 2009); self-injury as performance could be a manifestation of this function. Further research is necessary to ascertain whether this is typical of males who self-injure.

\subsection{Is participating in a study about self-injury harmful?}

One of the difficulties of researching NSSI is the iatrogenic potential of questions about self-injury. Talking about self-injury is no doubt distressing for some people, and given that self-injury is used to cope with distress, it is a concern that people may self-injure following research participation. Although there is little or no evidence to suggest this is the case, assumptions about the harmfulness of selfinjury research are commonplace and, at times, are even perpetuated by researchers themselves: 
Given the sensitive nature of the topic and the way the field has been dominated by quantitative approaches, it seemed clear that face-to-face interviews would be difficult to arrange and harrowing [emphasis added] for all participants. (Harris, 2000, p. 165)

This perspective is antithetical to how the participants in the current study evaluated their research participation (see section 6.3); certainly, none of their responses indicated that the process was the least bit harrowing.

While some of the participants did report feeling sad and upset as a result of taking part in this study, none regretted their participation. Instead, they stated that the research provided them with the opportunity to process their history of selfinjury in greater depth, as well as potentially help others who are experiencing similar difficulties. These reactions are consistent with a comprehensive review of 46 studies conducted by Jorm, Kelly, and Morgan (2007) who found that participants in mental health-related research were more likely to view their experience as positive, than negative. Furthermore, positive reactions to research participation were largely independent of distress, which provides evidence that people may rate research participation as positive even if they do experience some distress (Jorm et al., 2007).

However, participating in research specifically about self-injury may be distressing and, if so, the question remains whether that distress leads people to hurt themselves. Results from a recent study that examined the impact of screening questions about deliberate self-harm and suicidal thoughts on male secondary school students, showed that such questions did not significantly increase distress (Robinson et al., 2011). Only 9\% of students reported that these questions were moderately or very distressing, with at-risk students reporting higher distress levels (Robinson et al., 2011).

Aside from my research, only one other study to my knowledge has specifically examined whether questions about NSSI upset people who have selfinjured (Hanly, Pietrusza, Gluck, \& Whitlock, 2011). University students with a history of NSSI were twice as likely as students who had never self-injured to report that completing a survey, which included questions on NSSI, was difficult but that it 
encouraged them to reflect on their experiences (Hanly et al., 2011). Students with a history of NSSI were also almost three times as likely as those without such a history to report that completing the survey was a negative experience.

In both of these studies, some participants did experience distress as a result of answering questions about self-harm or self-injury, but neither study tracked participants over time. As such, it is impossible to determine whether students coped with their distress by self-injuring. Further research is needed to ascertain whether research about NSSI has an iatrogenic effect and, if so, whether this effect differs according to the research methodology (e.g., anonymous self-report survey versus in-depth interview) or the extent of NSSI questions. Certainly, the few negative comments received about the current study were primarily in regards to the DSHI (Gratz, 2001), which participants found overly detailed.

\subsection{Conclusion}

Based on the results of this study, the EAM (Chapman et al., 2006) is a useful theoretical framework for research and treatment purposes. In the majority of cases, it appeared that NSSI episodes did indeed serve an experientially avoidant function. However, more consideration of the role of positive reinforcement in maintaining self-injury is warranted. Furthermore, researchers and clinicians need to assess the interrelationships between cognitions (in particular, verbal rules) and emotions, to clarify the role of specific thought patterns in the reinforcement and maintenance of NSSI.

In the following chapter, I report the results of a national survey conducted to examine whether people with a recent history of NSSI endorse affect regulation as the primary function of self-injury and whether intrapersonal functions of NSSI are more commonly endorsed than interpersonal functions. Additionally, I investigate whether participants report a change in their affect or cognitions following engagement in NSSI. 


\section{CHAPTER FIVE}

\section{Quantifying the functions of self-injury}

\section{INTRODUCTION}

The Interpretative Functional Analysis presented in the previous chapter highlights the difficulty in functionally assessing individual episodes of NSSI. Despite this complexity, it is evident that self-injury did primarily fulfil an experientially avoidant function for the participants in my first study, thus supporting the overarching premise of the EAM (Chapman et al., 2006) and providing preliminary evidence for the utility of this model within Aotearoa New Zealand. However, both aversive emotions and cognitions served to establish NSSI as a form of escape or avoidance. Furthermore, several participants described experiencing positively reinforcing, intrapersonal consequences of NSSI (e.g., an adrenalin rush) in addition to negatively reinforcing, intrapersonal consequences (e.g., relief), which is consistent with previous research (Klonsky \& Glenn, 2009; Nock \& Prinstein, 2004).

Both negatively and positively reinforced intrapersonal consequences dominated participants' narratives about their most recent episode of self-injury; interpersonal consequences seldom featured. When interpersonal consequences were described, participants typically reported that these consequences occurred subsequent to intrapersonal ones. The time lapse between the self-injurious behaviour and the experience of interpersonal consequences makes it less likely that these consequences served as reinforcers for self-injurious behaviour (Anderson, 2007).

To build on the findings of the Interpretative Functional Analysis, I investigated whether similar functional processes could be identified in the selfinjurious episodes of a larger sample of people living in Aotearoa New Zealand. For the remaining two studies of my thesis, I therefore chose to focus on quantitatively 
testing the key assumptions of the EAM (Chapman et al., 2006), which are that NSSI functions as a negatively reinforced, experientially avoidant behaviour and that it belongs in a functional response class with other experientially avoidant behaviours (e.g., substance use).

For the current study, I first hypothesised that participants would endorse affect regulation as the primary function of NSSI (Klonsky, 2009; Klonsky \& Glenn, 2009). Second, I proposed that participants would endorse intrapersonal functions of NSSI more strongly than interpersonal functions (Klonsky \& Glenn, 2009; Nock \& Prinstein, 2004). My third hypothesis was that participants would report decreased negative affect and increased positive affect following NSSI (Klonsky, 2009). Finally, given the important role of self-referent cognitions in precipitating the self-injurious episodes I analysed in Chapter 4, I wanted to identify whether participants in the current study would report that the content of their cognitions had changed following their engagement in NSSI. Given that this has never been explicitly tested using quantitative measures, I had no a priori hypotheses as to whether shifts in cognitions would occur.

\section{METHOD}

\subsection{Procedure}

\subsubsection{Recruitment strategy}

Potential participants were invited to take part in a survey ${ }^{24}$ designed to investigate the thoughts, feelings, and events that lead people to intentionally injure themselves. I approached a number of community and mental health organisations and networks to ask them to post information about the study on their websites, weblogs, on notice boards in their offices or meeting rooms, and/or to include study information in their newsletters. The following organisations agreed to advertise or disseminate information about my study online, in print, and/or by placing posters on their notice boards: the Mental Health Foundation of New Zealand; Supporting

\footnotetext{
${ }^{24}$ Participants were informed that they could request a paper version of the survey if they did not want to complete it online.
} 
Families in Mental Illness (Auckland); Supporting Families Wairarapa; Supporting Families Otago; Supporting Families Taranaki; Male Survivors of Sexual Abuse Trust; Balance NZ; North Shore Women's Centre; Canterbury Men's Centre; Psychiatric Consumer Trust (Christchurch); Awareness (consumer group); Warmline (peer support organisation); Bipolar Support Canterbury; Regional Consumer Network (Auckland); Connect Supporting Recovery; Werry Centre; Eating Awareness Team (Christchurch); Auckland Sexual Abuse HELP; Kapiti Women's Centre; the Problem Gambling Foundation of New Zealand; West Coast Well Women's Centre; Rainbow Youth; and the Youth One Stop Shop (Palmerston North). Participants from my interview study who consented to be contacted about future research were also invited to complete the survey if they met the inclusion criteria.

I also recruited Psychology students from universities across Aotearoa New Zealand and gifted them each a $\$ 25$ prezzy card $^{25}$ to put up posters about my study around their campuses. Posters were placed at the Albany and Palmerston North campuses of Massey University, Auckland University, Auckland University of Technology, Waikato University, Canterbury University, and Otago University. I also put up posters advertising the study at various locations around the Kelburn campus of Victoria University.

Depending on the format of the advert (e.g., print/email newsletter or online), participants were either invited to type the survey address into their browser or click on the link within the advertisement to be directed to the survey. Online surveys were completed through Surveymonkey. Participants were not individually reimbursed for taking part but could opt into a draw to win one of two iPod Shuffles (2GB).

\footnotetext{
${ }^{25}$ Prezzy cards are gift cards which can be used at most stores that accept VISA.
} 


\subsubsection{Inclusion/exclusion criteria}

People were invited to participate in the study if they were 16 years of age or older, lived in Aotearoa New Zealand, and if they had injured themselves on purpose without suicidal intent, at least once in the past 12 months. They were excluded if they reported that their most recent episode of NSSI had occurred while they were experiencing psychosis (i.e., delusions or hallucinations) or mania.

\subsubsection{General ethical considerations}

Ethics approval for the current study was granted by the School of Psychology Human Ethics Committee at Victoria University of Wellington. Arguably, the most pressing ethical issue for the current study was the risk of participants becoming distressed as a result of survey completion and the potential for this distress to lead to self-injury. This risk was acknowledged in the information page that preceded the survey:

Non-suicidal self-injury can be a very difficult topic to answer questions about and there is a risk that some of the questions asked may bring up past memories or feelings that are unpleasant or distressing. If you do become distressed while completing the survey, you can stop filling it in at any time. Also, there will be a list of options for further support at the end of the survey.

Additional steps were taken to ensure the survey content was made explicit to participants so that they could make an informed choice as to whether to take part. For example, participants were informed that the survey contained lots of questions about NSSI and were given the following example: "You will be asked to think back to the time when you most recently injured yourself on purpose, without intending to kill yourself, and to answer questions about how you felt before and after that episode" (see Appendix H).

\subsubsection{Ethical issues specific to Internet Mediated Research}

Given that participation in the study involved completing an online survey, concerns specific to Internet Mediated Research (IMR; British Psychological Society, 2007) had to be addressed. The following three IMR issues were identified as pertinent to this research: ensuring that participants met the inclusion criteria, 
debriefing participants who exited the survey before finishing it, and potential breaches of confidentiality (British Psychological Society, 2007; Kraut et al., 2004).

\subsubsection{Meeting the inclusion criteria}

One of the risks associated with IMR is the inability to verify that participants meet the designated inclusion criteria (Kraut et al., 2004). To minimise the risk of people, who did not meet the inclusion criteria, participating in this study, question skip logic was used to direct respondents to specific pages based on their answers. When potential participants clicked on the survey link, they initially were directed to an information page about the study, which listed the inclusion/exclusion criteria. After clicking 'next', they were then directed to a second page which relisted the four inclusion/exclusion criteria and asked: "Are ALL FOUR of these statements true for you?" If respondents clicked yes, they were directed to the next page of the survey, but if they clicked no they were directed to a page that contained the following information: "Thank you for your time. Unfortunately, you do not meet the criteria to participate in this survey. Please close your browser (e.g., Internet Explorer, Firefox) to exit this survey."

\subsubsection{Exiting the survey before being debriefed}

Another risk of IMR is that respondents may choose not to finish the survey, preventing them from accessing any debriefing information because it is typically presented at the end of research participation (Kraut et al., 2004). To address this concern, when people confirmed that they met the inclusion criteria for the study they were required to enter their email address before being allowed to proceed to the survey questions. Participants were informed that their email addresses were compulsory in order to send them a copy of the debriefing information (see Appendix J).

This was not a sophisticated solution but survey functionality precluded the use of a pop-up debriefing window, which is a recommended safety strategy to ensure participants who close the survey early are adequately debriefed (British Psychological Society, 2007). Unfortunately, this method was not full-proof as one 
person entered an incorrect email address and another person simply typed in a series of letters.

\subsubsection{Protecting anonymity}

Participants were assured on the information page that their survey responses would remain anonymous and would not be linked to their email addresses. To ensure anonymity, once participants entered their email addresses and clicked 'next', they were directed to a separate survey where they were not required to enter any identifying information. Additionally, to protect participants' anonymity and privacy, their Internet Protocol addresses were not recorded.

\subsection{Participants}

\subsubsection{Flow of participants through the study}

Figure 5 depicts the flow of participants through the online version of the study. Some people $(N=45)$ indicated that they met the inclusion criteria but chose not to complete the survey. There are a number of reasons why people may have decided against proceeding to the survey questions. After reading the information sheet, they may have decided that filling in the survey would be upsetting or perhaps that they simply were not interested in taking part. Alternatively, the time required for survey completion, which was listed as being no more than 45 minutes, may have deterred people from participating. However, it is also possible that people may have revisited the site later when they did have the time available to complete the survey.

Requiring people to type in their email address before being directed to the survey questions may have been another deterrent. Although people were informed that their email addresses would not be linked to their survey answers, this may not have been sufficient to reassure some people that their responses would be anonymous. Given that self-injury is usually a secretive behaviour, other people may not have wanted their email addresses to be connected to a study about NSSI.

As is evident in Figure 5, the number of people $(N=197)$ who consented to participate in the survey by providing their email address was one less than the 


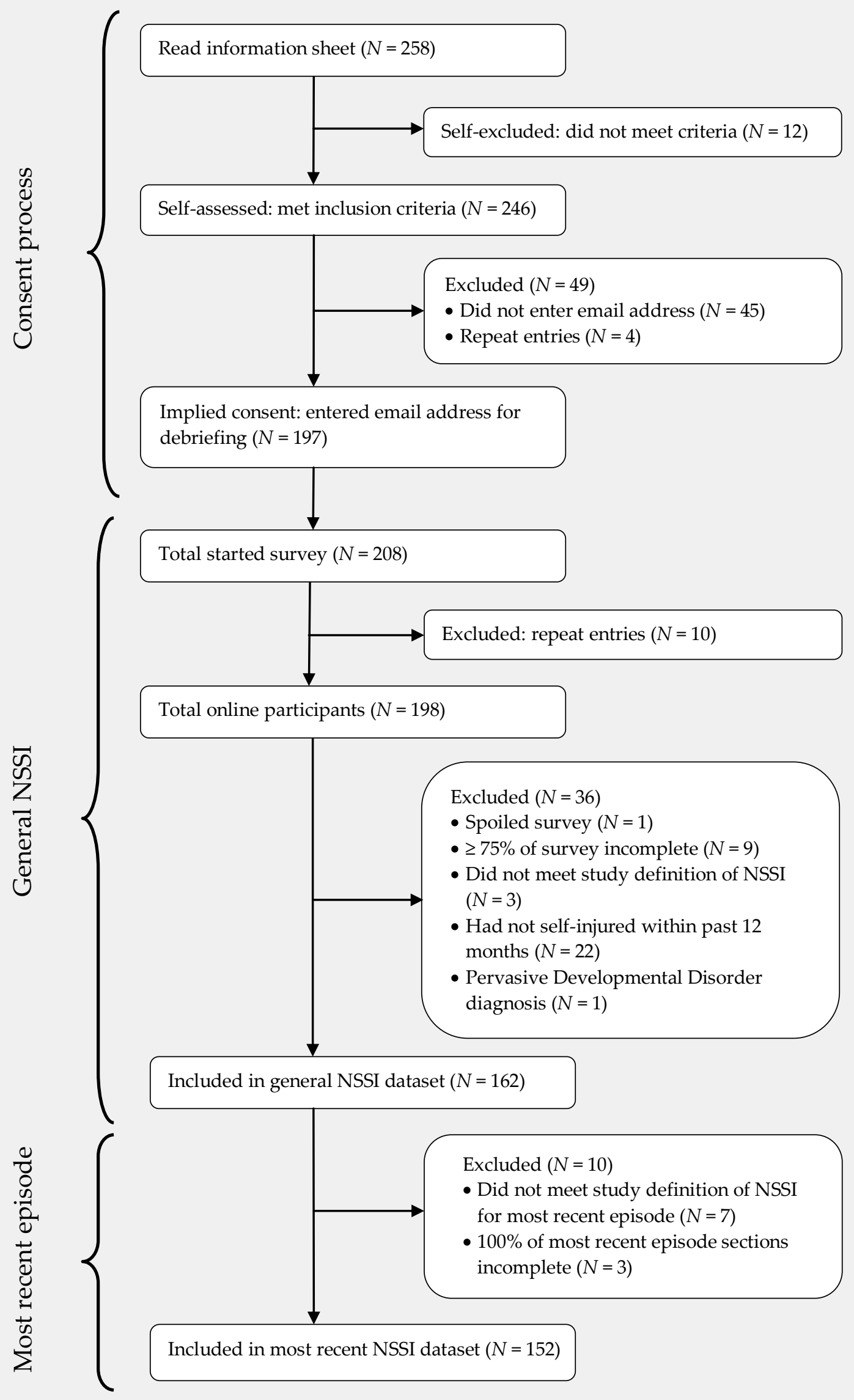

Figure 5. Flow of participants through the online survey. 
number of people $(N=198)$ who actually started the survey. Although this could be a repeat entry which I have not detected, this is unlikely because I thoroughly compared entries using demographic data (e.g., gender, age, and region) to ensure I removed multiple entries completed by the same people. Rather, it is possible that the question skip logic malfunctioned and directed one person through to the survey even though they had not entered their email address.

People were also informed that they could have a paper version of the survey if they preferred to fill in a hard copy. Only three people requested a paper copy of the survey and only one of these surveys was returned to me. This response was added to the dataset, which resulted in a total of 163 participants. All of the quantitative data analyses reported in this chapter were conducted using SPSS PASW Statistics (v. 18).

\subsubsection{Demographics and descriptive characteristics of the sample}

The participants ranged in age from 16 to 62 years $(\mathrm{M}=24.64, \mathrm{SD}=8.48)$ and were predominantly female (81.6\%), with $27(16.6 \%)$ male and $3(1.8 \%)$ transgender participants. Participants belonged to the following ethnic group(s): 134 (82.2\%) New Zealand European, 13 (8.0\%) Māori, 1 (0.6\%) Samoan, 3 (1.8\%) Chinese, 4 (2.5\%) Indian, and 28 (17.2\%) other ethnicities. People from every region in Aotearoa New Zealand participated in the study: 54 (33.1\%) from Auckland, 42 (25.8\%) from Wellington, 23 (14.1\%) from Canterbury, 18 (11.0\%) from Waikato, 10 (6.1\%) from Manawatu-Wanganui, 2 (1.2\%) from the Bay of Plenty, 9 (5.5\%) from Otago, and 1 $(0.6 \%)$ person each from Northland, the East Cape, Hawke's Bay, Marlborough, and Southland.

Over half of the sample (60.7\%) reported their current occupation as tertiary student, but there were a range of other occupations as well: 5 (3.1\%) were secondary school students, 13 (8.0\%) were in part-time employment, 21 (12.9\%) were in fulltime employment, 20 (12.3\%) were unemployed, 4 (2.5\%) were stay-at-home parents and $1(0.6 \%)$ person identified their occupation as other. The high number of tertiary students in the sample was reflected in the levels of education attained by 
participants: 9 (5.5\%) had no qualifications, 90 (55.2\%) had a high school qualification, $4(2.5 \%)$ had a trade/technical qualification, $38(23.3 \%)$ had a degree or diploma, $13(8.0 \%)$ had a postgraduate qualification, and $9(5.5 \%)$ had other qualifications.

Participants' sexual orientation, which was measured as a single-item variable based on Savin-Williams and Ream's (2007) conceptualisation, was as follows: 90 (55.2\%) heterosexual, 38 (23.3\%) mostly heterosexual, 15 (9.2\%) bisexual, 10 (6.1\%) mostly homosexual, 5 (3.1\%) homosexual, and 5 (3.1\%) asexual. Two thirds of the sample $(66.3 \%)$ reported receiving at least one mental health diagnosis, with the following diagnoses listed in order of highest frequency: 95 (58.3\%) Depression, 41 (25.2\%) Anxiety, 32 (19.6\%) PTSD, 24 (14.7\%) BPD, 16 (9.8\%) Bipolar Disorder, 15 (9.2\%) Anorexia, 14 (8.6\%) Bulimia, 9 (5.5\%) OCD, 5 (3.1\%) Substance Use Disorders, $1(0.6 \%)$ Schizophrenia, and $8(4.9 \%)$ other diagnoses.

Among those who endorsed being diagnosed with one or more mental health disorders, the number of diagnoses reported ranged from one to six, with an average of $2.41(\mathrm{SD}=1.28)$ diagnoses per person. This average may be slightly inflated due to the inclusion of PTSD and OCD as separate categories to anxiety disorders. The rationale for this demarcation was to ensure that people did not underreport these diagnoses if they were unaware that they qualify as anxiety disorders.

Finally, I used the first item of the Suicidal Behaviors Questionnaire-Revised (Osman et al., 2001) to assess lifetime suicidality (i.e., ideation and attempts). Participants were asked to choose one statement out of six that best applied to their experiences of suicidality. The majority of the participants had a history of suicidal ideation or behaviours; only $9(5.5 \%)$ had never thought about or attempted to kill themselves. Twenty-three (14.1\%) participants had experienced fleeting suicidal thoughts, $26(16.0 \%)$ had made a plan at least once to complete suicide but had not tried to kill themselves, $36(22.1 \%)$ had made a plan at least once and had really wanted to die, 25 (15.3\%) had attempted to kill themselves but did not want to die, 
while $44(27.0 \%)$ of the participants had attempted suicide and had really hoped to die.

\subsection{Measures}

\subsubsection{The Deliberate Self-harm Inventory}

A modified 15-item version of the DSHI (Gratz, 2001) was used to assess participants' lifetime prevalence of different types of NSSI. Modifications were made to shorten the measure and to address the issues that I discussed in the previous chapter (see pp. 101-105). Instead of asking about the age of onset, frequency, recency, and medical severity of each type of behaviour, I simply asked participants to endorse whether they had engaged in a particular behaviour, and, if yes, to then report the number of times they had engaged in that behaviour. Furthermore, after asking people how many times they had engaged in each type of self-injury I added: “If you can't remember, please estimate the number of times (e.g., 5, 10, 100) you have done this" (see Appendix H).

Other modifications included collapsing some of the questions into single items (Lundh et al., 2007). Specifically, questions two (which asks about burning yourself with a cigarette) and three (which asks about burning yourself with a lighter or match) were collapsed into a single question, which read as follows: "Have you ever intentionally (i.e., on purpose) burned yourself with a cigarette, lighter, or a match?" Similarly, questions four (which asks about carving words into your skin) and five (which asks about carving pictures, designs, or other marks into your skin) were collapsed into the following single question: "Have you ever intentionally (i.e., on purpose) carved words, pictures, designs, or other marks into your skin?" The word "comet" (item 10) was once again excluded.

\subsubsection{Inventory of Statements About Self-injury}

The ISAS (Klonsky \& Glenn, 2009) was chosen to assess the functions of participants' NSSI because it is the most comprehensive functional assessment measure of self-injury available. It consists of two sections, each of which can be used independently. Section one assesses the frequency of different types of NSSI 
and contextual factors surrounding these behaviours, while section two assesses the functions of non-suicidal self-injurious behaviours. Only section two was used in this study.

Section two of the ISAS is comprised of an index question (i.e., "When I selfharm, I am...") followed by 39 reasons for NSSI. Participants are invited to respond to each statement on a 3-point likert scale according to how relevant it is to their experience of NSSI (i.e., not relevant, somewhat relevant, or very relevant). The statements are grouped into the following 13 functions subscales ( 3 items per subscale): affect regulation (e.g., "...calming myself down"), interpersonal boundaries (e.g., "...creating a boundary between myself and others"), self-punishment (e.g., "...punishing myself"), self-care (e.g., "...giving myself a way to care for myself by attending to the wound"), anti-dissociation/feeling generation (e.g., "...causing pain so I will stop feeling numb"), anti-suicide (e.g., "...avoiding the impulse to attempt suicide"), sensation-seeking (e.g., "...doing something to generate excitement or exhilaration"), peer-bonding (e.g., “...bonding with peers"), interpersonal influence (e.g., "...letting others know the extent of my emotional pain"), toughness (e.g., “...seeing if I can stand the pain"), marking distress (e.g., "...creating a physical sign that I feel awful"), revenge (e.g., "...getting back at someone"), and autonomy (e.g., “...ensuring that I am self-sufficient").

For the purposes of this study, I changed the words "self-harm" to "selfinjury" in both the instructions for section two and the index question. The instructions thus read as follows: “This inventory was written to help us better understand the experience of non-suicidal self-injury. Below is a list of statements that may or may not be relevant to your experience of self-injury". The index question read as, "“When I self-injure, I am...". These modifications were made to ensure that the terminology (i.e., NSSI) that I used remained consistent throughout my survey. Given that the word self-harm typically refers to all self-injurious behaviours, regardless of suicide intent, using self-harm interchangeably with selfinjury may have confused participants and confounded these two phenomena. 
Although the ISAS was developed to measure the general functions of NSSI, I also wanted to examine the frequency and type of functions endorsed by participants for a single episode of NSSI. To achieve this, I invited participants to complete the ISAS twice at different points in the survey; once in relation to their general experiences of NSSI and once in relation to their most recent episode of NSSI. The instructions for the latter version were amended to reflect the requirement that participants answer only in relation to their most recent episode (see Appendix $\mathrm{H})$.

\subsubsection{Positive and Negative Affect Schedule}

The expanded form of the Positive and Negative Affect Schedule (PANAS-X; Watson \& Clark, 1994) was used to investigate whether participants reported changes in emotions following self-injury. The PANAS-X (Watson \& Clark, 1994) is a 60-item measure, with 13 scales that assess positive, negative, and other affect states. Items are rated on a 5-point scale from 1 (very slightly or not at all) to 5 (extremely).

There are two general dimension scales with 10 items in each: negative affect (afraid, scared, nervous, jittery, irritable, hostile, guilty, ashamed, upset, and distressed) and positive affect (active, alert, attentive, determined, enthusiastic, excited, inspired, interested, proud, and strong). The four basic negative emotion scales include: fear (afraid, scared, frightened, nervous, jittery, and shaky), hostility (angry, hostile, irritable, scornful, disgusted, and loathing), guilt (guilty, ashamed, blameworthy, angry at self, disgusted with self, and dissatisfied with self) and sadness (sad, blue, downhearted, alone, and lonely).

The three basic positive emotion scales are: joviality (happy, joyful, delighted, cheerful, excited, enthusiastic, lively, and energetic), self-assurance (proud, strong, confident, bold, daring, and fearless), and attentiveness (alert, attentive, concentrating, and determined). Finally, the other affective states included are: shyness (shy, bashful, sheepish, and timid), fatigue (sleepy, tired, sluggish, and drowsy), serenity (calm, relaxed, and at ease), and surprise (amazed, surprised, and astonished). As one of the most widely used measures of affect, the PANAS-X has 
been extensively tested and consistently demonstrates excellent psychometric properties (Watson \& Clark, 1994).

Although the PANAS-X (Watson \& Clark, 1994) can be applied to multiple time-frames (e.g., past week, in general), it is not typically used to evaluate the affective experience associated with a particular behaviour. However, as I discussed in Chapter 2, one of the limitations of self-injury research is the tendency for researchers to use unvalidated measures. It was therefore deemed preferable to use the PANAS-X and amend the instructions (see Appendix $H$ ), rather than create a new affect measure specifically for this study, especially in light of the observation by Watson and Clark (1994) that varying the time instructions does not appear to influence the scales' reliabilities. For the purposes of this study, I also modified the anchor point 1 to read not at all rather than very slightly or not at all as I wanted the lowest rating to reflect an absence of the emotion being measured.

\subsubsection{Revised Automatic Thoughts Questionnaire}

The revised Automatic Thoughts Questionnaire (ATQ-R; Kendall, Howard, \& Hays, 1989) is a 40-item measure that contains 30 negative self-statements (e.g., "I wish I were a better person") and 10 positive/neutral self-statements (e.g., “I'm proud of myself"). I included the ATQ-R in the survey because the role of negative thoughts in precipitating self-injurious behaviour is seldom investigated and such thoughts were identified as self-injury antecedents in the first study of this thesis.

The ATQ-R is designed to assess the frequency of automatic thoughts within the past week and, as such, using this measure to retrospectively identify thoughts before and after episodes of self-injury is unorthodox. However, in the absence of measures designed specifically for this purpose, I chose to use the ATQ-R for a series of exploratory analyses. Participants were asked to identify whether or not specific thoughts had occurred before and/or after their most recent episode of NSSI by simply responding yes or no. 


\subsection{Open-ended questions}

Participants were also asked a series of open-ended questions to enable them to describe the antecedents and consequences of their most recent episode of NSSI in their own words. This qualitative component was incorporated to add depth and scope to my understanding of the events that may establish the conditions for selfinjury and the consequences that may subsequently reinforce NSSI. To determine whether a specific event had led to their self-injury, they were asked: "Did something specific happen that led to this most recent episode of self-injury?" If they answered yes, then they were asked to describe what had happened, when it happened, and how it made them feel.

At the end of the survey, participants were asked to describe up to five consequences of their most recent episode of NSSI and to evaluate whether they had experienced these consequences as positive, negative, or neutral. Consequences were defined within the question as anything that had happened after the person injured themselves that was caused by the self-injury. That is, the consequences would not have occurred in the absence of the self-injurious episode. This definition is consistent with operant principles, which define behavioural consequences according to their impact on the environment (Sturmey et al., 2007a).

\section{QUANTITATIVE RESULTS}

\subsection{Characteristics of participants' self-injurious behaviour}

Despite simplifying the DSHI (Gratz, 2001) and explicitly stating that participants could provide estimates, some of the responses about the frequency and other types of NSSI were still challenging to interpret. Once again, I chose to err on the side of caution by including the lowest frequencies provided. For example, nonnumeric frequencies (e.g., countless) that were too difficult to quantify were treated as missing data; 10?, 10'ish, or 10+ were included in the data set as 10; 100-200 as 100; less than 10 as 9; a few times as 2; and hundreds of times as 100.

I also adhered as closely as possible to Gratz's (2001) definition of NSSI, which specifies that the behaviour results in tissue damage, when deciphering what 
qualified as a form of other NSSI. This was difficult because what 'counts' as NSSI varies considerably between studies, particularly in relation to self-poisoning (e.g., drinking toxic substances or overdosing) and hair-pulling. I chose to exclude both of these forms of self-injury; self-poisoning because of its ambiguous relationship to suicide (Brown et al., 2004; Freedenthal, 2007) and hair-pulling because it is currently classified within the DSM-IV-TR (American Psychiatric Association, 2000) as trichotillomania. Furthermore, people did not always state the severity of their selfpoisoning or hair-pulling behaviours and, as a result, it was unclear whether tissue damage resulted from these behaviours.

When reporting types of NSSI within the other category, some participants disclosed that tissue damage had occurred (e.g., "rubbed a key over my skin forming large graze", "slammed hand in a door repeatedly, intending to break it but only soft tissue injury"), while others listed behaviours where tissue damage was implied but not directly stated (e.g., "dislocated my fingers/thumbs", "poured cup of boiling water onto arms"), or highly probable because of the type of injury (e.g., "threw myself down the stairs", "swallowed glass") .

All behaviours where tissue damage was directly stated, implied, or highly probable were included. Behaviours where the occurrence of tissue damage was ambiguous (e.g., slapping oneself) were excluded. Finally, some participants reported other self-injury where the form, but not the implement used to carry out the injury, was listed in the DSHI. For example, the DSHI specifies burning oneself with a cigarette, lighter, or match. Some participants thus reported burning themselves with petrol, an iron, or hair straighteners in the other self-injury question. These responses were included in the dataset, which may have resulted in a slight inflation in the types of NSSI reported by participants.

\subsubsection{Global NSSI episodes}

The mean age of onset for NSSI was 14.45 years $(\mathrm{SD}=4.42)$ and ranged from 4 to 41 years of age. Participants reported having engaged in an average of 5.75 (SD = 2.66) different types of NSSI, although this ranged from one to 14 types. As can be 
Table 4

Frequencies of different types of NSSI

\begin{tabular}{|c|c|c|c|c|c|c|}
\hline \multirow{2}{*}{ NSSI type } & \multirow{2}{*}{$\begin{array}{c}N(\%) \text { reporting } \\
\text { NSSI type }\end{array}$} & \multicolumn{5}{|c|}{ Frequency of participants' engagement in NSSI types } \\
\hline & & Never & 1 time & 2-10 times & 11-50 times & $>50$ times \\
\hline Cutting wrists, arms, or other areas of body & $144(88.3 \%)$ & $19(11.7 \%)$ & $0(0.0 \%)$ & $39(23.9 \%)$ & $42(25.8 \%)$ & $62(38.0 \%)$ \\
\hline Severe scratching to extent of bleeding/scarring & $116(71.2 \%)$ & $46(28.2 \%)$ & $3(1.8 \%)$ & $59(36.2 \%)$ & $31(19.0 \%)$ & $20(12.3 \%)$ \\
\hline Sticking sharp objects into skin & $102(62.6 \%)$ & $60(36.8 \%)$ & $1(0.6 \%)$ & $64(39.3 \%)$ & $27(16.6 \%)$ & $7(4.3 \%)$ \\
\hline Carving words/pictures/designs/marks into skin & $97(59.5 \%)$ & $64(39.3 \%)$ & $13(8.0 \%)$ & $65(39.9 \%)$ & $16(9.8 \%)$ & $3(1.8 \%)$ \\
\hline Preventing wounds from healing & $98(60.1 \%)$ & $65(39.9 \%)$ & $1(0.6 \%)$ & $27(16.6 \%)$ & $36(22.1 \%)$ & $25(15.3 \%)$ \\
\hline Punching to extent of bruising & $86(52.8 \%)$ & $78(47.9 \%)$ & $9(5.5 \%)$ & $42(25.8 \%)$ & $21(12.9 \%)$ & $8(4.9 \%)$ \\
\hline Banging head to extent of bruising & $72(44.2 \%)$ & $90(55.2 \%)$ & $8(4.9 \%)$ & $42(25.8 \%)$ & $17(10.4 \%)$ & $2(1.2 \%)$ \\
\hline Burning with cigarette/lighter/match & $71(43.6 \%)$ & $92(56.4 \%)$ & $4(2.5 \%)$ & $46(28.2 \%)$ & $16(9.8 \%)$ & $3(1.8 \%)$ \\
\hline Biting to extent of breaking skin & $57(35.0 \%)$ & $105(64.4 \%)$ & $13(8.0 \%)$ & $30(18.4 \%)$ & $13(8.0 \%)$ & $1(0.6 \%)$ \\
\hline Rubbing glass into skin & $29(17.8 \%)$ & $133(81.6 \%)$ & $6(3.7 \%)$ & $15(9.2 \%)$ & $6(3.7 \%)$ & $2(1.2 \%)$ \\
\hline Rubbing sandpaper on body & $12(7.4 \%)$ & $149(91.4 \%)$ & $3(1.8 \%)$ & $7(4.3 \%)$ & $1(0.6 \%)$ & $1(0.6 \%)$ \\
\hline Dripping acid onto skin & $6(3.7 \%)$ & $155(95.1 \%)$ & $4(2.5 \%)$ & $1(0.6 \%)$ & $0(0.0 \%)$ & $0(0.0 \%)$ \\
\hline Breaking own bones & $5(3.1 \%)$ & $156(95.7 \%)$ & $4(2.5 \%)$ & $1(0.6 \%)$ & $0(0.0 \%)$ & $0(0.0 \%)$ \\
\hline Using bleach/ oven cleaner to scrub skin & $6(3.7 \%)$ & $157(96.3 \%)$ & $0(0.0 \%)$ & $4(2.5 \%)$ & $0(0.0 \%)$ & $1(0.6 \%)$ \\
\hline
\end{tabular}

Note. $N$ reporting NSSI type may not equal frequencies of engagement as some participants endorsed type of NSSI but did not report frequency. Frequencies may not add up to $100 \%$ because of missing data. 
seen in Table 4, the most common form of self-injury was cutting, which was endorsed by $88.3 \%$ of the participants, followed by severe scratching to the extent of bleeding or scarring (71.2\%); sticking sharp objects into one's skin (62.6\%); and carving words, pictures, designs, or other marks into one's skin (59.5\%). The least common forms of self-injury were dripping acid onto one's skin, breaking one's own bones, and using bleach or oven cleaner to scrub one's skin; only $3 \%$ of the participants endorsed engaging in these types of NSSI one or more times.

Additionally, 37 (22.7\%) of the participants reported having engaged in at least one other form of self-injury, such as swallowing glass, punching walls, and dislocating fingers. Similarly to the DSHI (Gratz, 2001) data presented in Chapter 4, the frequencies of NSSI behaviours in the current study were grouped into five categories (see Table 4).

\subsubsection{Most recent NSSI episode}

To assess how participants had self-injured most recently, they were presented with a list of self-injurious behaviours identical to those in the modified version of the DSHI (Gratz, 2001), which was described in section 2.4.1, and asked to endorse any behaviours that applied to their episode. They were asked to identify whether the injury had been severe enough to require medical treatment, if they told anyone that they had self-injured, and whether they had used drugs or alcohol prior to self-injuring. The frequencies of NSSI types for participants' most recent episode of self-injury are presented in Table 5.

Participants reported engaging in a range of NSSI types for their most recent episode of self-injury, with the most common type being cutting (65.6\%), followed by severe scratching (21.5\%) and carving words, pictures, designs, or other marks into the skin (16.0\%). The least commonly endorsed types of NSSI were biting $(4.3 \%)$, rubbing sandpaper on the body (1.2\%), and scrubbing the skin with bleach or oven cleaner $(0.6 \%)$. A further six (3.7\%) people reported engaging in other forms of NSSI (e.g., punching walls) for their most recent episode. No-one endorsed injuring 
themselves most recently by rubbing glass into, or dripping acid onto, their skin or by breaking their own bones.

Table 5

Frequencies of different types of NSSI for participants' most recent episode

\begin{tabular}{lc}
\hline NSSI type & $\boldsymbol{N}(\mathbf{\%})$ \\
\hline Cutting wrists, arms, or other areas of body & $107(65.6 \%)$ \\
Severe scratching to extent of bleeding/scarring & $35(21.5 \%)$ \\
Carving words/pictures/designs/marks into skin & $26(16.0 \%)$ \\
Punching to extent of bruising & $20(12.3 \%)$ \\
Sticking sharp objects into skin & $16(9.8 \%)$ \\
Preventing wounds from healing & $16(9.8 \%)$ \\
Banging head to extent of bruising & $13(8.0 \%)$ \\
Burning with cigarette/lighter/match & $10(6.1 \%)$ \\
Biting to extent of breaking skin & $7(4.3 \%)$ \\
Rubbing sandpaper on body & $2(1.2 \%)$ \\
Using bleach/oven cleaner to scrub skin & $1(0.6 \%)$ \\
Rubbing glass into skin & $0(0.0 \%)$ \\
Dripping acid onto skin & $0(0.0 \%)$ \\
Breaking own bones & $0(0.0 \%)$ \\
\hline
\end{tabular}

Note. Frequencies total more than $100 \%$ because some participants reported using multiple types of NSSI during their most recent episode.

On average, people reported using $1.69(\mathrm{SD}=1.04)$ types of self-injury during their most recent episode; this ranged from one to six types. The mean number of days that had elapsed between participants completing the survey and their most recent episode of NSSI was $81.06(S D=85.20)$ with a range of zero to 359 days. Approximately $15 \%$ of participants reported that their injury was severe enough to require medical treatment but it is unknown whether they actually received treatment.

Almost a third of the participants (30.1\%) told someone that they had hurt themselves on purpose; 20 (12.3\%) participants told their mental health clinician (e.g., psychologist, social worker), 19 (11.7\%) told a friend, and seven (4.3\%) told their partner or spouse. A few participants told their health professional (3.7\%), boyfriend/girlfriend (2.5\%), others such as an online friend (2.5\%), a co-worker $(1.8 \%)$, parent $(1.8 \%)$, sibling $(1.2 \%)$, or another relative, teacher/lecturer, or 
acquaintance (all $0.6 \%$ ). The majority of participants did not consume more than two standard drinks of alcohol $(74.8 \%)$, take in excess of a recommended dosage of medication (85.9\%), or use illegal drugs (91.4\%) before injuring themselves.

\subsection{Is affect regulation the primary function of NSSI?}

Descriptive statistics and reliability coefficients for the ISAS (Klonsky \& Glenn, 2009) in reference to participants' global and most recent NSSI episodes are presented in Table 6. I have tabulated these results side-by-side to allow for ease of comparison, but will discuss the results for the global and most recent episodes of NSSI separately.

\subsubsection{Self-reported functions of participants' global NSSI episodes}

As is evident from the skewness and kurtosis values presented in Table 6, visual inspection of the histograms and Q-Q plots of each of the 13 ISAS subscales indicated that none of the data was normally distributed. Rather, the majority of the subscales were skewed to the right. Kolmogorov-Smirnov tests with Lilliefor's correction (Field, 2009) confirmed that all subscales were significantly non-normal, $D^{\prime} s(162-163) \geq .13\left(p^{\prime} s<.001\right)$. Given that the assumption of normality was violated for all subscales, non-parametric statistics were used.

Prior to beginning the non-parametric analyses, I calculated the means and standard deviations of each of the subscales to allow for comparisons with other studies. Affect regulation ( $\mathrm{M}=4.65, \mathrm{SD}=1.61)$, self-punishment $(\mathrm{M}=4.17, \mathrm{SD}=1.90)$, and marking distress $(\mathrm{M}=2.80, \mathrm{SD}=1.97)$ were the most highly endorsed functions, while sensation-seeking $(M=0.75, S D=1.17)$, revenge $(M=0.66, S D=1.22)$, and peer-bonding $(\mathrm{M}=0.15, \mathrm{SD}=0.57)$ were the least endorsed functions.

Comparable results were obtained through other measures of central tendency - the median and mean rank - that do not rely on the assumption of normality. For example, affect regulation and self-punishment both had the highest median of 5 with mean ranks of 11.43 and 10.94 respectively. The third most endorsed item, marking distress, had a median of 3 and a mean rank of 8.98 . 
Table 6

Descriptive statistics and reliability coefficients for the ISAS in reference to participants' global and most recent episodes of NSSI

\begin{tabular}{|c|c|c|c|c|c|c|c|c|c|c|c|c|}
\hline \multirow[b]{2}{*}{$\begin{array}{l}\text { ISAS subscale } \\
\text { (score range 0-6) }\end{array}$} & \multicolumn{6}{|c|}{ Global episodes $(N=163)^{*}$} & \multicolumn{6}{|c|}{ Most recent episode $(N=140)$} \\
\hline & Mean (SD) & Median & $\begin{array}{c}\text { Cronbach's } \\
\alpha \\
\end{array}$ & Skewness & Kurtosis & $\begin{array}{l}\text { Mean } \\
\text { Rank }\end{array}$ & Mean (SD) & Median & $\begin{array}{c}\text { Cronbach's } \\
\alpha \\
\end{array}$ & Skewness & Kurtosis & $\begin{array}{l}\text { Mean } \\
\text { Rank } \\
\end{array}$ \\
\hline Affect regulation & $4.65(1.61)$ & 5.00 & .73 & $-1.23^{* *}$ & 0.72 & $11.43^{\mathrm{a}}$ & $4.61(1.72)$ & 5.00 & .74 & $-1.22^{* * *}$ & 0.60 & $11.48 \mathrm{~g}$ \\
\hline Self-punishment & $4.17(1.90)$ & 5.00 & .82 & $-.08^{* *}$ & -0.56 & $10.94^{\mathrm{a}}$ & $3.94(2.13)$ & 5.00 & .88 & $-0.72^{* * *}$ & -0.87 & $10.51 \mathrm{~g}$ \\
\hline Marking distress & $2.80(1.97)$ & 3.00 & .78 & $0.14^{* *}$ & -1.12 & $8.98^{\mathrm{b}}$ & $2.68(1.96)$ & 2.00 & .73 & $0.28^{* * *}$ & -1.05 & $9.31^{\mathrm{h}}$ \\
\hline $\begin{array}{l}\text { Anti-dissociation/ } \\
\text { feeling generation }\end{array}$ & $2.70(2.17)$ & 3.00 & .86 & $0.20^{* *}$ & -1.34 & $8.56^{\mathrm{b}}$ & $2.10(2.27)$ & 1.00 & .91 & $0.62^{* * *}$ & -1.12 & $7.70^{\mathrm{h}}$ \\
\hline Anti-suicide & $2.23(1.97)$ & 2.00 & .84 & $0.47^{* *}$ & -0.83 & $7.71^{\mathrm{b}}$ & $2.03(2.27)$ & 1.00 & .93 & $0.67^{* * *}$ & -1.01 & $7.50^{\mathrm{h}, \mathrm{i}}$ \\
\hline Self-care & $1.50(1.48)$ & 1.00 & .60 & $0.81^{* *}$ & -0.26 & $6.69^{c}$ & $1.36(1.53)$ & 1.00 & .65 & $1.07^{* * *}$ & 0.54 & $6.92^{\mathrm{i}, \mathrm{j}}$ \\
\hline Toughness & $1.28(1.54)$ & 1.00 & .73 & $1.13^{* *}$ & 0.42 & $6.21^{c}$ & $1.06(1.55)$ & 0.00 & .85 & $1.51^{* * *}$ & 1.68 & $6.30 \mathrm{i}$ \\
\hline $\begin{array}{l}\text { Interpersonal } \\
\text { influence }\end{array}$ & $1.17(1.46)$ & 0.00 & .68 & $1.06^{* *}$ & 0.24 & $6.00^{c, d}$ & $0.90(1.32)$ & 0.00 & .70 & $1.59^{* * *}$ & 2.20 & $6.02^{\mathrm{j}, \mathrm{k}, 1}$ \\
\hline $\begin{array}{l}\text { Interpersonal } \\
\text { boundaries }\end{array}$ & $1.16(1.51)$ & 1.00 & .77 & $1.40^{* *}$ & 1.51 & $6.02^{c, d}$ & $0.88(1.47)$ & 0.00 & .80 & $1.81^{* * *}$ & 2.66 & $5.79 \mathrm{j}, \mathrm{k}, 1$ \\
\hline Autonomy & $0.77(1.17)$ & 0.00 & .68 & $1.50^{* *}$ & 1.42 & $5.06^{\mathrm{d}, \mathrm{e}}$ & $0.62(1.20)$ & 0.00 & .79 & $2.41^{* * *}$ & 6.21 & $5.40^{\mathrm{k}, 1}$ \\
\hline Sensation-seeking & $0.75(1.17)$ & 0.00 & .59 & $1.78^{* *}$ & 3.37 & $5.12^{\mathrm{d}, \mathrm{e}}$ & $0.53(1.20)$ & 0.00 & .79 & $2.75^{* * *}$ & 7.88 & $4.97 \mathrm{k}, 1$ \\
\hline Revenge & $0.66(1.22)$ & 0.00 & .82 & $2.02^{* *}$ & 3.94 & $4.77 \mathrm{e}$ & $0.60(1.36)$ & 0.00 & .91 & $2.50^{* * *}$ & 5.76 & $5.04 \mathrm{k}, 1$ \\
\hline Peer-bonding & $0.15(0.57)$ & 0.00 & .63 & $4.90^{* *}$ & 27.00 & 3.52 & $0.16(0.84)$ & 0.00 & .92 & $5.97^{* * *}$ & 36.90 & 4.07 \\
\hline
\end{tabular}

Note. $\quad *$ All the data in the global episodes section of the table was based on 163 participants apart from the Autonomy subscale which was based on 162 participants.

** All D's $(162-163)>.13$, p's <.001; *** All $D^{\prime}$ s $(140)>.15$, p's <.001.

a-1 Mean ranks with the same superscripts indicate subscales that do not differ significantly from one another. All other subscales were significantly different. 
Sensation-seeking, revenge, and peer-bonding all had medians of 0 and low mean ranks. The subscales demonstrated questionable to good internal consistency ( $\alpha$ ranged from .60 for self-care to .86 for anti-dissociation/feeling generation), with the exception of sensation-seeking which demonstrated poor internal consistency ( $\alpha$ $=.59)$.

To determine whether there were any significant differences between the ISAS subscales, I conducted analyses using the Friedman Test $(N=162)$ and post-hoc Wilcoxon signed-rank tests (i.e., non-parametric alternatives to ANOVA and t-tests). Results indicated that at least one of the subscales was significantly different from at least one other subscale, $\chi^{2}(12)=855.36, p<.001$. Given the number of post-hoc comparisons, a Bonferroni corrected alpha of $\mathrm{p}<.001$ was used to control for familywise error. The results of these comparisons are reported in Table 6.

Affect regulation and self-punishment, although not different to each other, differed significantly from all of the other subscales. Marking distress, anti-suicide, and anti-dissociation/feeling generation did not differ, but were all significantly different to the other 10 subscales. Self-care was not significantly different to toughness, interpersonal influence, and interpersonal boundaries, while autonomy was not significantly different to interpersonal influence, interpersonal boundaries, sensation-seeking, and revenge. Peer-bonding was the only subscale that was significantly different to all of the other subscales. Effect sizes were calculated by dividing $Z$ by the square root of the number of observations (Field, 2009). For significantly different subscales, the effect sizes ranged from $r=.21$ (anti-suicide compared with self-care) to $r=.61$ (affect regulation compared with peer-bonding).

\subsubsection{Self-reported functions of participants' most recent NSSI episode}

Visual inspection of the data (i.e., histograms and Q-Q plots), along with the means and standard deviations of the ISAS subscales for participants' most recent episode of NSSI, showed that it was predominantly skewed to the right. The skewness and kurtosis values for each of the subscales are presented in Table 6; the 
distributions of all of these subscales were significantly non-normal, $D^{\prime} s(140) \geq .15$ ( $\left.\mathrm{p}^{\prime} \mathrm{s}<.001\right)$. While the means and mean ranks for the functions of participants' most recent episode were similar to those of the global functions they endorsed, the medians tended to be slightly lower. Lower medians are to be expected given that the participants were rating the functions of one specific episode of NSSI as opposed to their general experience of self-injury.

The most highly endorsed functions for global episodes of NSSI - affect regulation, self-punishment, and marking distress - were also the most highly endorsed functions for participants' most recent episode. Mean ranks for affect regulation and self-punishment were 11.48 and 10.51 respectively, and they both had medians of five. Marking distress, the third most endorsed function, had a mean rank of 9.31 and a substantially lower median of 2. Peer bonding was once again the least endorsed function with a mean rank of 4.07 and a median of 0 . Sensationseeking (mean rank $=4.97$, median $=0)$ and revenge $($ mean rank $=5.04$, median $=0)$ followed peer-bonding as the second and third least endorsed functions.

This pattern of results differed only slightly from that obtained in relation to participants' global episodes of NSSI, where sensation-seeking was the third, not the second, least endorsed item. Apart from this discrepancy, the order in which the functions were endorsed was identical for participants' global and most recent episodes of NSSI. The internal consistencies of the ISAS subscales for participants' most recent episode of self-injury tended to be higher than for global episodes, and ranged from questionable ( $\alpha=.65$ for self-care) to excellent ( $\alpha=.93$ for anti-suicide).

The Friedman Test was used to determine whether there were any significant differences between the functions endorsed for participants' most recent NSSI episode. Results showed a significant difference in participants' $(N=140)$ endorsement of the subscales, $\chi^{2}(12)=697.74, \mathrm{p}<.001$. Post-hoc Wilcoxon signedrank tests with a Bonferroni corrected alpha of $\mathrm{p}<.001$ revealed a similar pattern of results to that obtained for global episodes of NSSI (see Table 6). 
Peer-bonding was once again the only subscale that differed significantly from all of the other subscales. Affect regulation and self-punishment did not differ from each other, but differed significantly from the other eleven subscales. Marking distress, anti-suicide, and anti-dissociation/feeling generation were not significantly different to one another but did differ from the other subscales. Although self-care once again was not significantly different to toughness, interpersonal influence, or interpersonal boundaries, it also did not differ from anti-suicide, which contrasted with the difference observed between self-care and anti-suicide when the participants completed the ISAS in reference to their global episodes of NSSI. The lack of differences between autonomy, interpersonal influence, interpersonal boundaries, sensation-seeking, and revenge was also observed for participants' most recent episode of NSSI. Effect sizes for the significant differences between functions ranged from $r=.21$ (autonomy compared with toughness) to $r=.62$ (revenge compared with self-punishment).

\subsection{Are intrapersonal functions more highly endorsed than interpersonal?}

Self-reported functions of NSSI have been found to load onto two superordinate factors that reflect intrapersonal and interpersonal reasons for selfinjury (Klonsky \& Glenn, 2009; Nock \& Prinstein, 2004). Grouping the singlefunction models on the basis of intrapersonal and interpersonal functions is theoretically consistent with multi-function models such as the FFM (Nock \& Prinstein, 2004, 2005) and the EAM (Chapman et al., 2006), and allows researchers to test these models. Unfortunately, conducting a confirmatory factor analysis with data that is significantly non-normal, such as the functions data collected in this study, is not recommended (Tabachnik \& Fidell, 2007).

Instead, I conducted cluster analyses, which do not require data to be normally distributed (Norusis, 2010), to determine whether the single function subscales clustered into intrapersonal and interpersonal categories. The purpose of conducting a cluster analysis is: 
to group entities on the basis of their similarity with respect to selected variables, so that members of the resulting groups are as similar as possible to others within their group (high within-group homogeneity) and as different as possible to those in other groups (low between-group homogeneity). (Clatworthy, Buick, Hankins, Weinman, \& Horne, 2005, p. 330)

For the current study, I conducted two hierarchical agglomerative cluster analyses using Ward's method. Squared Euclidean distance was used as the distance measure in both analyses (Norusis, 2010).

\subsubsection{Cluster analyses}

\subsubsection{Cluster analysis of global NSSI functions}

Inspection of the agglomeration schedule (presented in Table 7) and dendrogram (see Figure 6) for the global functions of NSSI provided evidence of a two cluster solution. The first cluster (agglomeration coefficient $=2095.13$, stage 8 of Table 7) contained the following eight subscales: peer-bonding, revenge, sensationseeking, interpersonal influence, autonomy, interpersonal boundaries, toughness, and self-care.

Table 7

Agglomeration schedule, Ward's method and squared Euclidean distance for global NSSI functions

\begin{tabular}{|c|c|c|c|c|c|c|}
\hline \multicolumn{7}{|c|}{ Agglomeration schedule } \\
\hline \multirow[b]{2}{*}{ Stage } & \multicolumn{3}{|c|}{ Cluster combined } & \multicolumn{3}{|c|}{ Stage cluster first appears } \\
\hline & Cluster 1 & Cluster 2 & Coefficients & Cluster 1 & Cluster 2 & Next stage \\
\hline 1 & 8 & 9 & 127.00 & 0 & 0 & 2 \\
\hline 2 & 8 & 12 & 294.67 & 1 & 0 & 5 \\
\hline 3 & 1 & 5 & 478.17 & 0 & 0 & 4 \\
\hline 4 & 1 & 13 & 727.33 & 3 & 0 & 6 \\
\hline 5 & 6 & 8 & 982.17 & 0 & 2 & 8 \\
\hline 6 & 1 & 10 & 1326.25 & 4 & 0 & 8 \\
\hline 7 & 3 & 11 & 1695.75 & 0 & 0 & 11 \\
\hline 8 & 1 & 6 & 2095.13 & 6 & 5 & 12 \\
\hline 9 & 2 & 4 & 2521.13 & 0 & 0 & 10 \\
\hline 10 & 2 & 7 & 3115.13 & 9 & 0 & 11 \\
\hline 11 & 2 & 3 & 4234.03 & 10 & 7 & 12 \\
\hline 12 & 1 & 2 & 7871.85 & 8 & 11 & 0 \\
\hline
\end{tabular}


The second cluster (agglomeration coefficient $=4234.03$, stage 11 of Table 7 ) contained the following five subscales: affect regulation, self-punishment, antidissociation/feeling generation, anti-suicide, and marking distress. Combining these two clusters into one cluster increased the squared agglomeration coefficient from 4234.03 to 7871.85 (see stage 12 of Table 7 ); the size of this increase supports a two cluster solution.

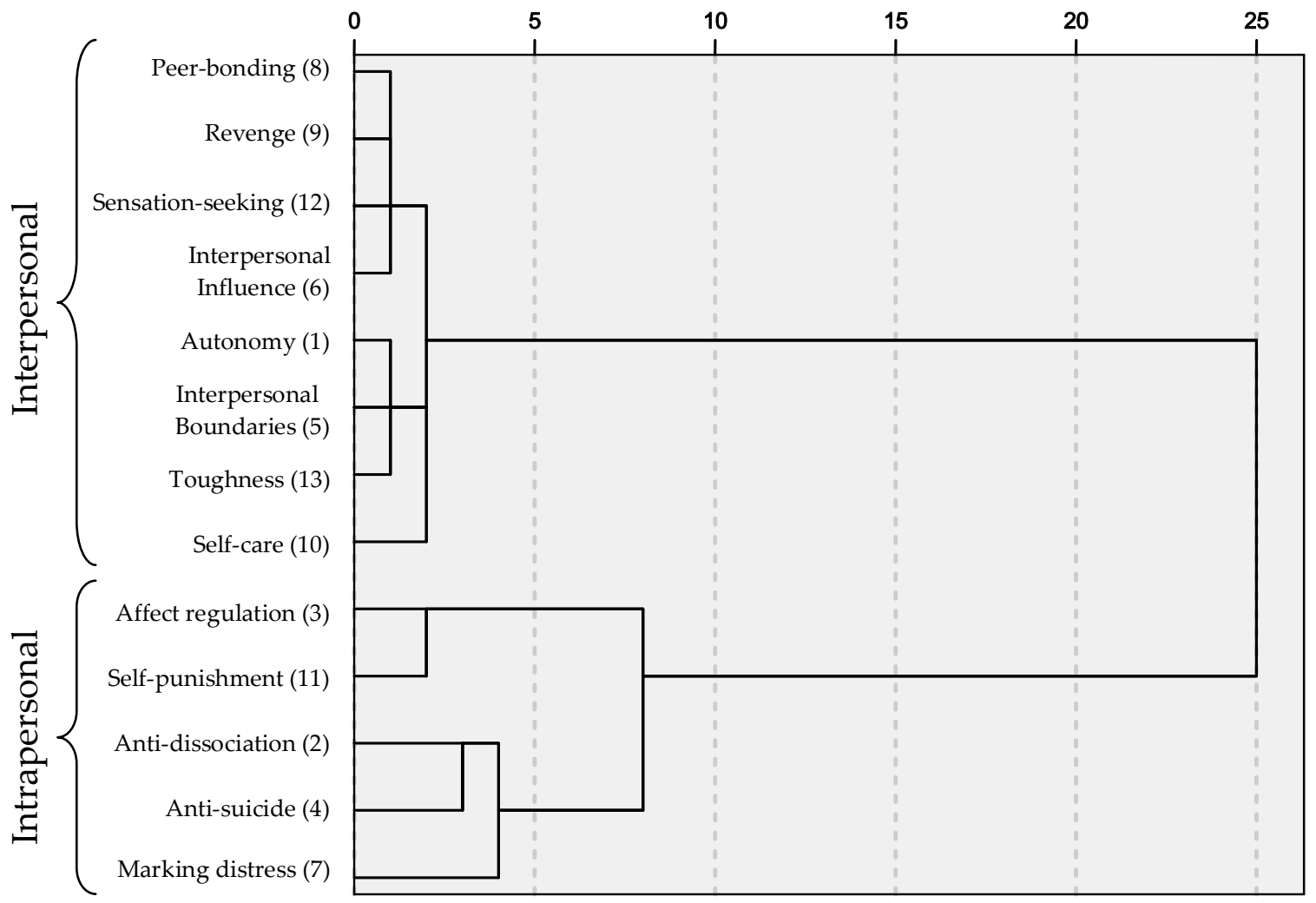

Figure 6. Dendrogram using Ward linkage for functions of participants' global episodes of NSSI.

The grouping of variables in the two clusters is consistent with previous research (Klonsky \& Glenn, 2009; Nock \& Prinstein, 2004) in that the first cluster contains subscales that reflect interpersonal functions, while the second cluster contains subscales that reflect intrapersonal functions. The only subscale that does not fit neatly into this intrapersonal/interpersonal dichotomy is self-care, which should theoretically cluster with the intrapersonal functions, but instead has clustered with the interpersonal functions. 


\subsubsection{Cluster analysis of participants' most recent NSSI episode}

The ISAS subscales for the functions of participants' most recent episode of self-injury demonstrated an identical two cluster solution to that found for the global NSSI functions, with the exception of self-care, which moved from the first to the second cluster. Evidence for the two cluster solution is presented in Table 8 and Figure 7. The first cluster (agglomeration coefficient $=981.43$, stage 6 of Table 8 ) contained the following seven subscales: peer bonding, sensation-seeking, autonomy, interpersonal boundaries, toughness, interpersonal influence, and revenge.

\section{Table 8}

Agglomeration schedule, Ward's method and squared Euclidean distance for functions of participants' most recent NSSI episode

\begin{tabular}{|c|c|c|c|c|c|c|}
\hline \multicolumn{7}{|c|}{ Agglomeration schedule } \\
\hline & \multicolumn{3}{|c|}{ Cluster combined } & \multicolumn{3}{|c|}{ Stage cluster first appears } \\
\hline Stage & Cluster 1 & Cluster 2 & Coefficients & Cluster 1 & Cluster 2 & Next stage \\
\hline 1 & 8 & 12 & 86.50 & 0 & 0 & 2 \\
\hline 2 & 1 & 8 & 198.00 & 0 & 1 & 4 \\
\hline 3 & 6 & 9 & 331.00 & 0 & 0 & 6 \\
\hline 4 & 1 & 5 & 517.25 & 2 & 0 & 5 \\
\hline 5 & 1 & 13 & 717.40 & 4 & 0 & 6 \\
\hline 6 & 1 & 6 & 981.43 & 5 & 3 & 12 \\
\hline 7 & 7 & 10 & 1314.43 & 0 & 0 & 10 \\
\hline 8 & 3 & 11 & 1704.43 & 0 & 0 & 11 \\
\hline 9 & 2 & 4 & 2116.43 & 0 & 0 & 10 \\
\hline 10 & 2 & 7 & 2676.93 & 9 & 7 & 11 \\
\hline 11 & 2 & 3 & 4063.76 & 10 & 8 & 12 \\
\hline 12 & 1 & 2 & 6970.15 & 6 & 11 & 0 \\
\hline
\end{tabular}

The second cluster (agglomeration coefficient $=4063.76$, stage 11 of Table 8 ) contained the following six subscales: affect regulation, self-punishment, marking distress, self-care, anti-dissociation/feeling generation, and anti-suicide. Combining these two clusters into one cluster results in a substantial increase in the agglomeration coefficient from 4063.76 to 6970.15 (see stage 12 of Table 8), which supports a two cluster solution. These two clusters are once again consistent with 
previous research on the distinction between the intrapersonal and interpersonal functions of NSSI.

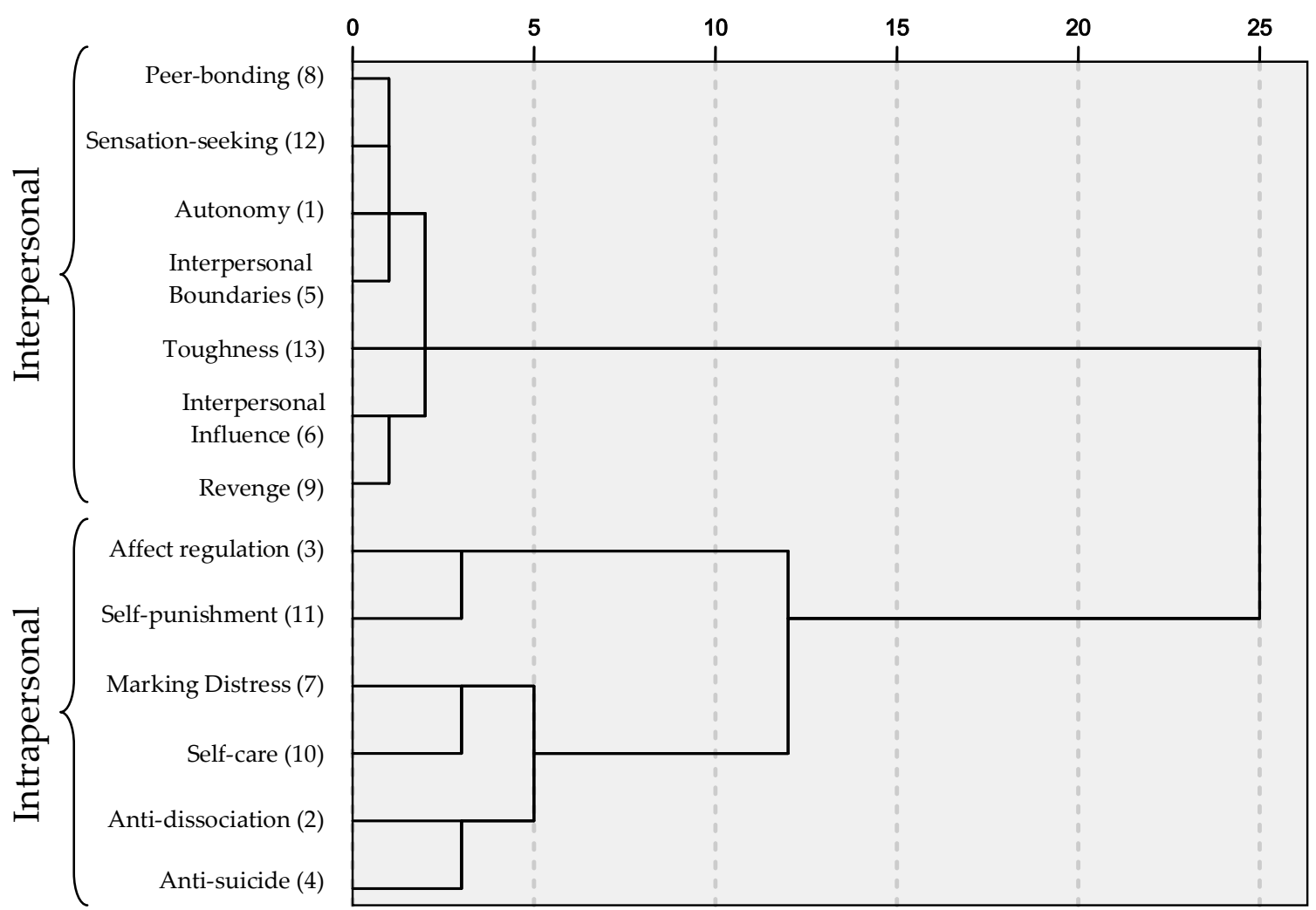

Figure 7. Dendrogram using Ward linkage for functions of participants' most recent NSSI episode.

\subsubsection{Comparing intrapersonal and interpersonal functions}

Guided by previous research (Klonsky \& Glenn, 2009; Nock \& Prinstein, 2004) and based on the results of the cluster analyses, four new variables were created. Participants' responses for the subscales within each of the four clusters (i.e., the two clusters from each cluster solution) were summed and then averaged to derive the following four scores: global intrapersonal functions (affect regulation, selfpunishment, anti-dissociation/feeling generation, anti-suicide, and marking distress), global interpersonal functions (peer bonding, revenge, sensation-seeking, interpersonal influence, autonomy, interpersonal boundaries, toughness, and self-care), most recent episode intrapersonal functions (affect regulation, self-punishment, marking distress, self-care, anti-dissociation/feeling generation, and anti-suicide), and most recent episode interpersonal functions (peer bonding, sensation-seeking, autonomy, 
interpersonal boundaries, toughness, interpersonal influence, and revenge). Internal consistency was questionable for the global intrapersonal $(\alpha=.63)$ and global interpersonal $(\alpha=.68)$ functions, and acceptable for the most recent episode intrapersonal functions $(\alpha=.70)$ and most recent episode interpersonal functions $(\alpha=$ .79).

Before beginning the analyses to determine whether there were significant differences between the intrapersonal and interpersonal functions for global and most recent episodes, I once again examined the histograms and Q-Q plots of the data to check whether the distributions were normal. Both the global intrapersonal functions variable $(\mathrm{M}=3.31, \mathrm{SD}=1.23$, Median = 3.40) and most recent intrapersonal functions variable $(\mathrm{M}=2.79, \mathrm{SD}=1.26$, Median = 2.67) were normally distributed. However, both global interpersonal functions variable $(\mathrm{M}=0.94, \mathrm{SD}=0.72$, Median $=$ $0.75)$ and most recent interpersonal functions variable $(\mathrm{M}=0.68, \mathrm{SD}=0.85$, Median $=$ 0.43) were positively skewed. Normality tests confirmed that these variables were significantly non-normal; for the global interpersonal functions variable, $D(163)=$ $.14, \mathrm{p}<.001$, and for the most recent interpersonal functions variable, $D(140)=.22$, $\mathrm{p}<.001$

Given that two out of the four variables of interest were significantly nonnormal, Wilcoxon signed-rank tests were used to analyse whether there was a significant difference between the global intrapersonal and interpersonal functions, and the intrapersonal and interpersonal functions for participants' most recent episode. Participants' endorsement of intrapersonal versus interpersonal functions for their global experiences of NSSI was significantly different, $T=118.00, p<.001$, $r=.60$. More specifically, 152 participants endorsed intrapersonal functions more highly than interpersonal functions, nine participants endorsed interpersonal functions more highly than intrapersonal functions, and two participants did not demonstrate any difference in their endorsement of either intrapersonal or interpersonal functions. 
There was also a significant difference in participants' endorsement of intrapersonal versus interpersonal functions for their most recent experience of NSSI, $T=263.50, \mathrm{p}<.001, r=.58$. Out of 140 participants, 130 participants endorsed intrapersonal functions more highly than interpersonal functions, nine participants endorsed interpersonal functions more highly than intrapersonal functions, and one participant did not demonstrate any difference in their endorsement of either intrapersonal or interpersonal functions.

Although intrapersonal functions were more commonly endorsed, 111 participants (79.3\%) out of the 140 participants reported self-injuring most recently for both intrapersonal and interpersonal reasons. Only 28 participants (20.0\%) reported engaging in their most recent episode of NSSI for exclusively intrapersonal reasons and one participant $(0.7 \%)$ reported self-injuring most recently for exclusively interpersonal reasons.

\subsection{Do people report a decrease in negative affect and an increase in positive affect}

\section{following NSSI?}

To assess whether participants would retrospectively report decreased negative affect and increased positive affect following self-injury, they were asked to complete the PANAS-X (Watson \& Clark, 1994) twice; once in relation to how they felt before their most recent episode of NSSI and once in relation to how they felt after their most recent episode of NSSI. Responses were averaged to create composite scores for each participant for the following 13 PANAS-X subscales: negative affect, positive affect, fear, hostility, guilt, sadness, joviality, self-assurance, attentiveness, shyness, fatigue, serenity, and surprise (Watson \& Clark, 1994). Since participants completed the PANAS- $X$ twice, they each had a total of 26 scores.

Visual inspection of the histograms and Q-Q plots showed that, apart from negative affect before the episode, all of the scales appeared to violate the normality assumption. Specifically, most of the scales were positively skewed. KolmogorovSmirnov tests with Lilliefors correction confirmed that the distributions of all of the scales were significantly non-normal, $D^{\prime} s(142-149) \geq .08\left(\mathrm{p}^{\prime} \mathrm{s}<.05\right)$, with the exception 
Table 9

Descriptive statistics and reliability coefficients for the PANAS-X subscale responses before and after participants' most recent episode of NSSI

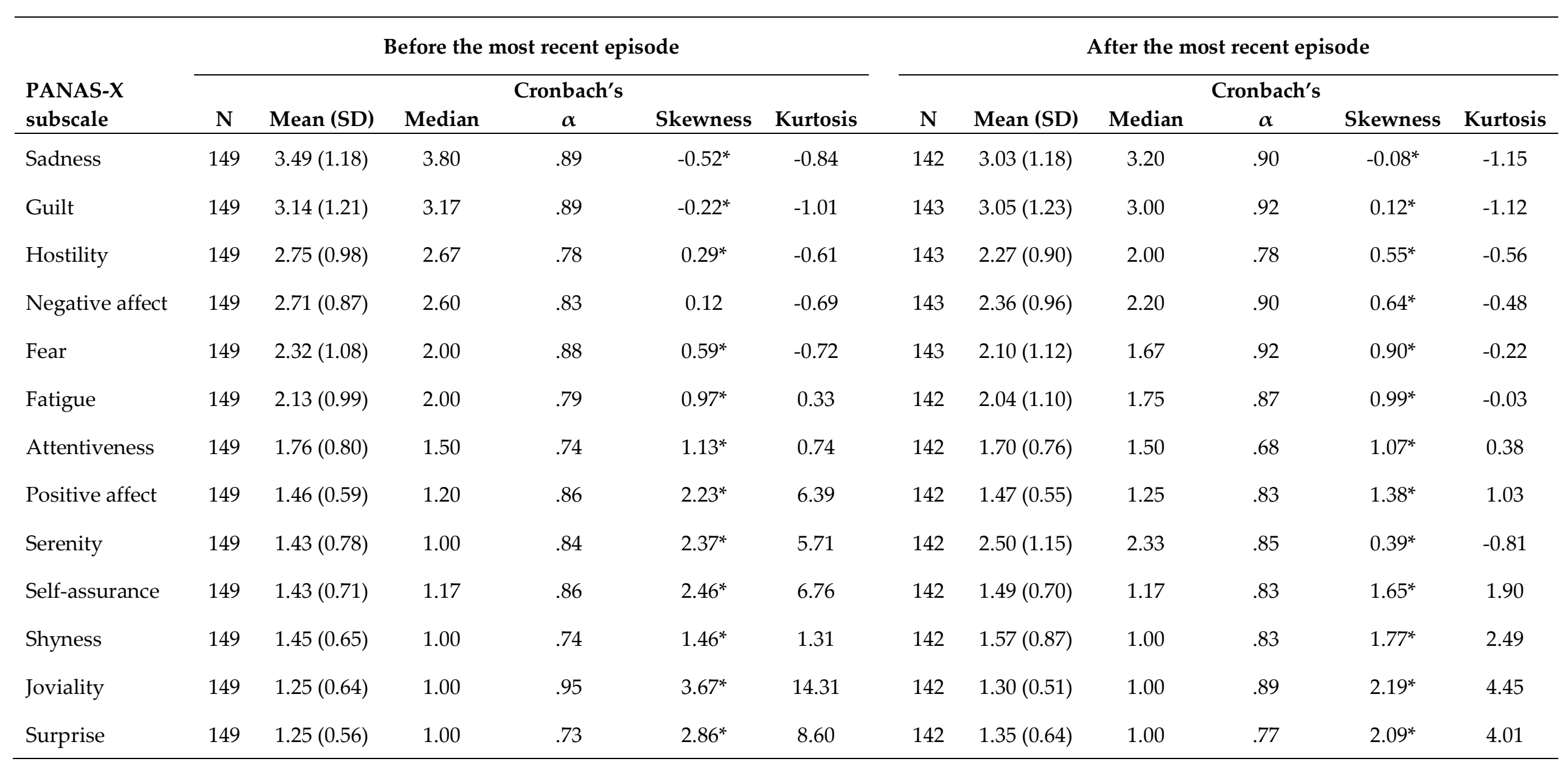

Note. ${ }^{*}$ All $D^{\prime} \mathrm{s}(142-149)>.08, \mathrm{p}^{\prime} \mathrm{s}<.05$. 
of negative affect before the episode. Descriptive statistics and reliability coefficients for the PANAS-X scales are presented in Table 9.

Wilcoxon signed-rank tests showed that negative affect $(T=2433.00, \mathrm{p}<.001$, $r=.30)$, hostility $(T=1750.00, \mathrm{p}<.001, r=.35)$, sadness $(T=1625.50, \mathrm{p}<.001, r=.33)$, and fear $(T=2113.50, \mathrm{p}<.001, r=.21)$ decreased significantly after participants had self-injured, whereas there was no significant change in feelings of guilt ( $T=4021.50$, $\mathrm{p}=.17, r=.06)$. In comparison, serenity $(T=715.50, \mathrm{p}<.001, r=.45)$ and joviality $(T=$ 1045.00, $\mathrm{p}<.05, r=.14)$ increased significantly after self-injury, but there was no significant change in general positive affect $(T=2755.00, \mathrm{p}=.34, r=.02)$.

The results for the remaining five subscales of the PANAS-X should be considered exploratory given that I had no a priori hypotheses as to whether these emotions would change following NSSI. Shyness $(T=765.00, \mathrm{p}<.01, r=.16)$ increased significantly and surprise $(T=388.50, \mathrm{p}<.01, r=.16)$ decreased significantly following NSSI, but there was no significant changes in fatigue $(T=2405.50, \mathrm{p}=.17, r=.08)$, attentiveness $(T=2386.00, \mathrm{p}=.42, r=.05)$, or selfassurance $(T=1507.00, \mathrm{p}=.28, r=.06)$.

Although it is useful to know which affect states changed significantly following participants' engagement in NSSI, these statistics do not provide an indication of how many people experienced such changes. However, the positive, negative, and tie ranks for each of the subscales are in effect change scores, which can be used to determine how many participants experienced increases, decreases, or no change in particular emotional states following their episode of self-injury. The ranks for each of the PANAS-X subscales are presented in Table 10.

Although the majority of participants experienced decreased negative affect (69.32\%), hostility (68.53\%), sadness (62.69\%), and fear (54.55\%) following NSSI, there were a number of participants whose negative emotions increased and others who experienced no change. For example, $23.08 \%$ of people reported feeling more hostile following self-injury and $8.39 \%$ reported no change in their levels of hostility. 
Variable rates of endorsement were also observed for what can be considered neutral or positive affect states. An overwhelming majority of participants $(75.35 \%)$ reported an increase in serenity after they had self-injured, but some participants' (9.15\%) experience of serenity decreased, while others (15.49\%) experienced no change. In contrast, more participants $(45.77 \%)$ reported that their experience of joviality did not change after self-injuring, than those who reported an increase $(37.32 \%)$ or decrease $(16.90 \%)$ in joviality.

Table 10

Ranks for PANAS-X subscales before and after participants' most recent episode of NSSI

\begin{tabular}{|c|c|c|c|}
\hline \multirow[b]{2}{*}{ PANAS- $X$ subscale } & \multicolumn{3}{|c|}{ Ranks } \\
\hline & $\begin{array}{l}\text { Negative - decrease } \\
\text { after NSSI ( } \% \text { of } N)\end{array}$ & $\begin{array}{l}\text { Positive - increase } \\
\text { after NSSI ( } \% \text { of N) }\end{array}$ & $\begin{array}{c}\text { Tie - no change } \\
\text { after NSSI ( } \% \text { of } N)\end{array}$ \\
\hline Negative Affect & 99 (69.23) & 40 (27.97) & $4(2.80)$ \\
\hline Hostility & $98(68.53)$ & $33(23.08)$ & $12(8.39)$ \\
\hline Sadness & 89 (62.68) & 34 (23.94) & 19 (13.38) \\
\hline Fear & 78 (54.55) & 38 (26.57) & 27 (18.88) \\
\hline Guilt & $71(49.65)$ & $62(43.36)$ & $10(6.99)$ \\
\hline Fatigue & 66 (46.48) & 40 (28.17) & $36(25.35)$ \\
\hline Attentiveness & $56(39.44)$ & 46 (32.39) & 40 (28.17) \\
\hline Positive Affect & $52(36.62)$ & 55 (38.73) & 35 (24.65) \\
\hline Self-assurance & 37 (26.06) & 46 (32.39) & $59(41.55)$ \\
\hline Joviality & 24 (16.90) & 53 (37.32) & 65 (45.77) \\
\hline Shyness & $24(16.90)$ & 45 (31.69) & 73 (51.41) \\
\hline Surprise & 17 (11.97) & $35(24.65)$ & $90(63.38)$ \\
\hline Serenity & $13(9.15)$ & 107 (75.35) & 22 (15.49) \\
\hline
\end{tabular}

Note. All $N=142-143$. Modal findings are in bold.

\subsection{Does the content of people's cognitions change following NSSI?}

To examine whether there was any change in participants' self-reported cognitions following a NSSI episode, I summed and then averaged participants' scores on the negative and positive/neutral items from the ATQ-R (Kendall et al., 1989). This resulted in four new variables for each participant: negative cognitions 
before most recent episode, negative cognitions after most recent episode, positive/neutral cognitions before most recent episode, and positive/neutral cognitions after most recent episode. Internal consistency for negative cognitions before $(\alpha=.94)$ and after $(\alpha=.95)$ self-injury was excellent, while internal consistency for positive/neutral cognitions before $(\alpha=.80)$ and after $(\alpha=.74)$ self-injury was acceptable to good.

Visual inspection of histograms and Q-Q plots showed that the negative cognitions data was skewed to the left, while the positive/neutral cognitions data was skewed to the right. Kolmogorov-Smirnov tests with Lilliefor's correction $\left(D^{\prime} \mathrm{s}(140-142)>.12\right.$, all $\left.\mathrm{p}^{\prime} \mathrm{s}<.001\right)$ confirmed that all of the subscales were significantly non-normal, necessitating the use of non-parametric tests to determine whether there were significant changes in participants' self-reported negative or positive/neutral cognitions following engagement in self-injury.

Wilcoxon signed-rank tests showed that negative cognitions $(T=1906.00, p<$. $001, r=.31)$ decreased significantly and positive cognitions $(T=777.00, \mathrm{p}<.001, r=.31)$ increased significantly following NSSI. More specifically, 91 (64.54\%) participants reported a decrease in negative cognitions following NSSI, 36 (25.53\%) reported an increase in negative cognitions, and 14 (9.93\%) reported no change. Less conclusive findings were evident with positive cognitions as although 74 (52.86\%) participants reported that they experienced an increase and 16 (11.43\%) experienced a decrease in positive/neutral cognitions following NSSI, 50 (35.71\%) participants reported no change in their positive/neutral cognitions.

\subsection{Summary of quantitative findings}

The results supported the hypotheses in that affect regulation was the most highly endorsed function of both participants' global and most recent episodes of NSSI; however, there was no significant differences in the level of participants' endorsement for affect regulation or self-punishment. Consistent with the high endorsement of affect regulation and self-punishment, intrapersonal functions were more highly endorsed than interpersonal functions for both global and most recent 
episodes of NSSI. Specific negative affect states were reported to decrease significantly following NSSI, but only one positive affect state (i.e., joviality) increased. Although serenity increased significantly, this is classified by Watson \& Clark (1994) under the rubric of other affective states. Participants similarly reported a significant decrease in negative cognitions and a significant increase in positive cognitions after they had self-injured most recently. Before discussing the implications of these findings, I present the qualitative analyses of participants' descriptions of the antecedents and consequences of their most recent episode of self-injury.

\section{OPEN-ENDED RESPONSES}

To add depth and scope to my understanding of the events that establish the conditions for, and potentially reinforce, self-injurious behaviours, participants were given the opportunity to describe, in their own words, what had led to their most recent episode of NSSI and the consequences of that episode. To code the antecedents of the NSSI episodes, a coding system for aversive events, based on the stressful event categories listed in the Unpleasant Events Schedule (Lewinsohn, Mermelstein, Alexander, \& MacPhillamy, 1983), was developed for the purposes of this study (see Appendix I). The consequences described by participants were analysed using Thematic Analysis (Braun \& Clarke, 2006).

\subsection{Antecedents of NSSI}

Out of 153 participants, $96(58.9 \%)$ reported that something specific had led to their most recent episode of self-injury. These events were coded within the following 10 categories: health and wellbeing (focused on physical health); achievement-academic-job; domestic, day-to-day inconveniences; interpersonal relationships; legal; material-financial; death-related; other; no specific event; and insufficient information to code the event. Events could be coded in multiple categories. A Clinical Psychologist and I coded all the events; interrater agreement was $82.86 \%$ and the final ratings for any discrepant items were determined following discussion. Table 11 shows the number of participants who reported events in each 
Table 11

Antecedent event categories with percentage endorsement and qualitative examples

\begin{tabular}{|c|c|c|}
\hline Event category & N (\%) & Examples of event descriptions \\
\hline Health and well-being & $7(6.60)$ & $\begin{array}{l}\text { - I had a lot to drink and was sick of not feeling loved and wanted and a guy was being nice to me for once in my life. } \\
\text { Next thing you know me and him are in the parking lot down the road from a club and he completely uses me and it } \\
\text { hurt really bad and then ran off afterwards with me bawling my eyes out. (Female, 19) } \\
\text { - Binging. (Female, 20) }\end{array}$ \\
\hline Achievement-academic-job & $12(11.32)$ & $\begin{array}{l}\text { - Being pressured at work so when I got home I went out for a smoke and burned myself with it. (Female, 28) } \\
\text { - Occurred during exam period. Was punishing myself because I wasn't studying and because I hoped the adrenaline } \\
\text { would keep me awake. (Female, 19) }\end{array}$ \\
\hline Interpersonal relationships & $59(55.66)$ & $\begin{array}{l}\text { - I had had an argument with my mother, and left my parental house very distressed. I wanted to drive to my husband's } \\
\text { work so I could talk to him (it was near the end of his shift), but was too upset to drive properly. So I drove to the } \\
\text { nearest parking lot and cut my left arm repeatedly with a razor blade that I keep in my car. (Female, 23) } \\
\text { - After a messy break up caused by a cheating girlfriend, the (now) ex girlfriend told me that she had power over me, and } \\
\text { that she could hurt me more than anything or anyone else could. (Male, 19) }\end{array}$ \\
\hline Material-financial & $2(1.89)$ & - In debt rang study link who won't pay any more. (Female, 50) \\
\hline Death-related & $3(2.83)$ & $\begin{array}{l}\text { - Close friend died in June. (Female, 19) } \\
\text { - It was the around the time of my mother's death. (Female, 19) }\end{array}$ \\
\hline Other & $2(1.89)$ & $\begin{array}{l}\text { - Had a shower and hated the sight of myself. (Female, 42) } \\
\text { - Had a discussion on religion, and I came to realise that I have not been a good believer. (Female, 21) }\end{array}$ \\
\hline No specific event & $14(13.21)$ & $\begin{array}{l}\text { - Built up negative emotions that had come to a point of not being able to deal with them anymore. (Female, 19) } \\
\text { - I just felt very misunderstood. (Female, 27) }\end{array}$ \\
\hline $\begin{array}{l}\text { Insufficient information to } \\
\text { code the event }\end{array}$ & $7(6.60)$ & $\begin{array}{l}\text { - Abandonment. (Female, 32) } \\
\text { - } \text { Memories of traumatic events that happened on that date years before. (Female, 17) }\end{array}$ \\
\hline
\end{tabular}


category, with accompanying examples. No-one reported that their most recent episode of NSSI was precipitated by domestic, day-to-day inconveniences or legal events. As a result, these two categories are excluded from Table 11.

The majority of the antecedents (55.66\%) described by participants were categorised as interpersonal relationship events, which typically included fights or arguments with friends or family, relationship break-ups, or being mistreated by others. The second highest category of events was concerned with failure to achieve goals, academic struggles, or job stress.

\subsection{Thematic Analysis of the consequences of NSSI}

When participants were asked to describe up to five consequences of their most recent episode, 122 people identified experiencing at least one consequence, with an average number of $2.59(\mathrm{SD}=1.38)$ consequences per person. ${ }^{26}$ Three people identified consequences but did not evaluate whether these were positive, negative, or neutral; as a result, these responses could not be further analysed. Of the 313 consequences that were included in the thematic analysis, $101(32.27 \%)$ were evaluated by participants as positive, $170(54.31 \%)$ as negative, and $42(13.42 \%)$ as neutral.

For the thematic analysis, I analysed the consequences that were positively or negatively evaluated, rather than consequences that were rated as neutral. Two distinct themes were identified within this data - self becomes transgressor and self becomes helper - each of which had a number of sub-themes (see Figure 8). Becoming a transgressor necessitated concealing the transgressions from others, being judged by others for the transgressions, and causing others to suffer, while becoming a helper was enacted through regulating emotions, accessing support and/or treatment, and the physical wound.

\footnotetext{
26 Three responses were discarded because participants had typed in "none" or "nothing at all" and then rated these responses as neutral.
} 


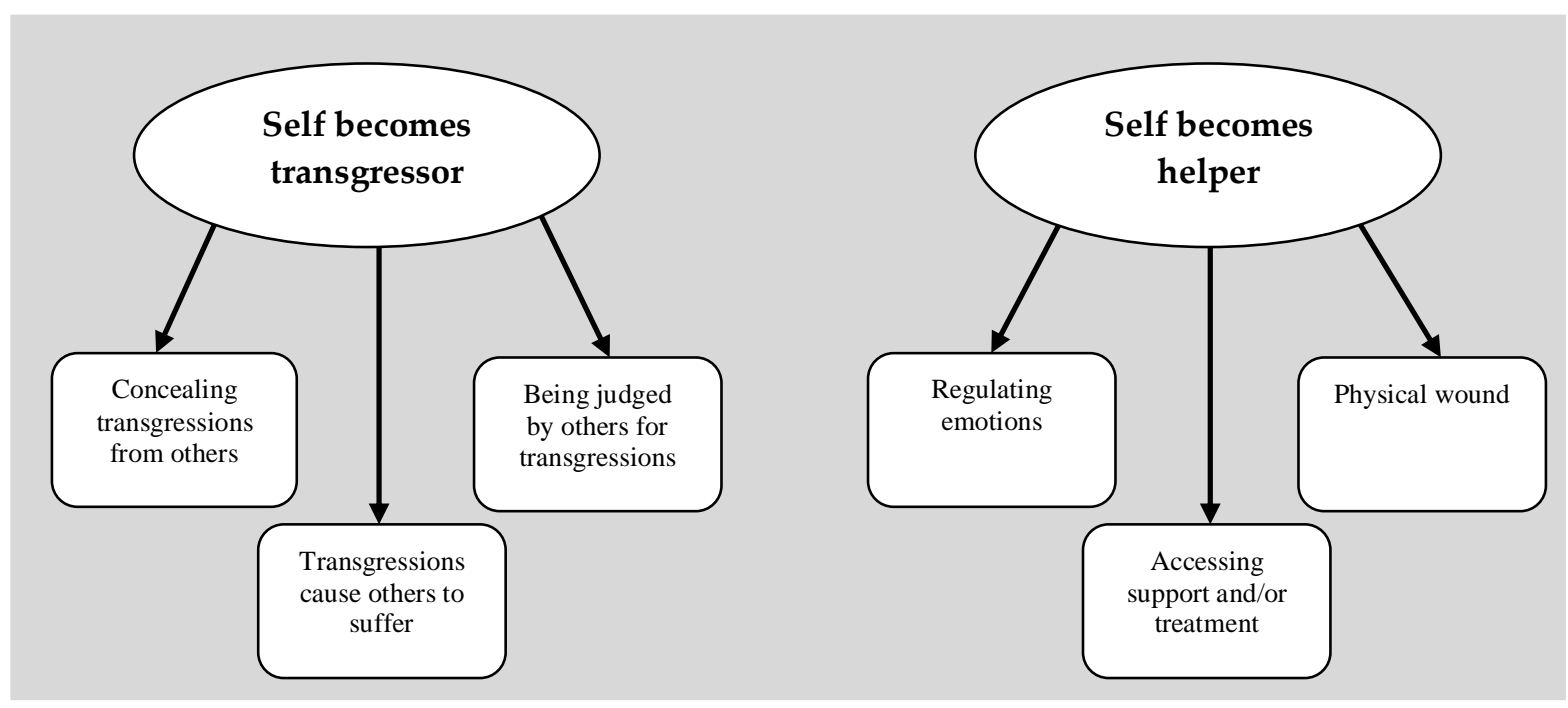

Figure 8. Thematic Map.

\subsubsection{Self becomes transgressor}

Self-injury is an anathema to many people, who struggle to understand why someone would purposefully cut, burn, or otherwise damage their own skin (Strong, 2000). As identified by two participants ${ }^{27}$, injuring oneself in such a way contravenes pervasive Western cultural discourses of protecting, preserving, and extending the life of one's body and clearly transgresses social mores:

I felt guilty at what I had done because I had damaged my body. (Female, 18)

I felt disgusted with myself, that I was harming my body which should be something precious, was burdened with this guilt for quite some time. (Female, 19)

In contrast to non-Western cultures where self-injury can signify socially desirable psychological and physical transitions (e.g., initiation rites) (Favazza, 1996), amongst Pākehā living in Aotearoa New Zealand self-injury typically signifies psychopathology and an attendant inability to cope with problems, in the words of one participant, "like a 'normal' person".

Participants readily judged themselves as abnormal - that is, as transgressors of social norms-because they had self-injured:

I felt like everyone would notice and think I was crazy. (Female, 18)

${ }^{27}$ Any spelling or major grammatical errors within responses have been corrected to facilitate readability. Furthermore, the way in which the quotes are presented differs from how I presented the interview quotes (see Chapter 4 ) as the responses in this study were anonymous. 
What's wrong with me...why did I slip? It had been awhile since I self harmed, what does it mean that I have done this again? (Female, 22)

That such judgements occurred in the context of particular self-referential emotions (e.g., guilt, shame) is unsurprising given that experiencing these emotions necessitates processes of self-representation and -evaluation, both of which are strongly influenced by prevailing community norms (Zinck, 2008):

These emotions specifically contribute to highlighting the difference between one's own and another's perspective, coordinate the subject's behavior in a social environment, promote her integration with the social group and support the mediation between specific individual and social goals. They further promote an adjustment of the self-concept in relation to the feedback of a social environment and to an internal evaluation of behavior and thoughts according to the subject's own standard. (Zinck, 2008, p. 498)

In this way, self-referential emotions function to communicate and regulate individuals' identities and self-concepts (Zinck, 2008).

Given that self-referential emotions cannot occur in the absence of selfevaluation, the reporting of these emotions as negative consequences of self-injury demonstrates that participants have judged themselves as transgressors. However, for those participants who simply listed negative emotions - guilt, shame, embarrassment - as consequences, it is impossible to determine why the act of selfinjury had made them feel guilty or ashamed, or more specifically, exactly what personal and social values they believed they had broken.

Other people, however, did provide explanations that revealed the selfevaluative component of these emotions in more detail. For several participants, the feelings of guilt and shame stemmed from the fact that they had hurt themselves again:

I felt bad that I could not resist the compulsion to do it. (Female, 29)

Guilt for self-injuring when I told myself I wouldn't. (Female, 18)

I felt like a failure for a long time because I let myself down by relapsing into self harming again. (Female, 19)

Incredible amount of shame at resorting to old coping strategies to cope. (Female, 44)

Feeling extremely disappointed in myself because I was supposed to have stopped SI-ing. (Female, 17) 
The inability to resist self-injury and utilise healthier coping strategies was evaluated by these participants as a personal failing, which impacted on the way that they perceived themselves and had the potential to impact on the way that they were perceived by others.

Indeed, having others find out about the self-injury was explicitly identified by some participants as a source of shame:

Shame in having my flatmates see the cuts on my arms. (Female, 22)

I had to mention it to two of my friends, which was hard. I felt embarrassed because I had not cut myself in at least a year. (Transgender, 26)

The shame of my parents finding out what I had done. (Female, 41)

Given that self-injury is perceived as shameful, having to prevent others from finding out about their transgressions was one of the most commonly reported negative consequences of self-injury.

\subsubsection{Concealing transgressions from others}

Many people described that having to hide the physical evidence of their selfinjury from others was a negative consequence of their behaviour:

More scars to hide. (Female, 39)

Having to hide new cuts. (Female, 17)

Had to cover it up. (Female, 17)

Having to hide wounds from my boyfriend. (Female, 24)

I had to be sure not to let the wound/scar show. (Female, 27)

Ensuring those around them did not find out that they had self-injured had both practical and psychological implications. On a practical level, participants' clothing choices were restricted because they had to keep their wounds or scars covered:

The wound was on a place on my arm which could be seen by others if I wore a T-shirt. I had to wear long sleeves while it healed even when it was hot to cover it up. I don't want my flatmates or friends to know I've been struggling. I now have to continue wearing long sleeves or use concealer if I want to wear short sleeves. (Female, 27)

My arm had to be covered at all times to hide what I had done while it healed. (Female, 20) 
Couldn't wear short sleeves so I was boiling hot all summer. (Female, 21)

I couldn't wear shorts. (Female, 35)

On a psychological level, having to guard against being found out impacted negatively on participants:

The constant fear of it being seen or someone realising what it was. Puts you more on edge than you were beforehand. (Female, 19)

I didn't talk to anyone the next few days because I stressed that people might somehow notice I was hurt and ask why. (Transgender, 19)

Nervous that someone would find out. (Female, 18)

Jeans stuck to my leg, had to be pried off, slight paranoia that people would notice the slight off-colour of them. (Female, 20)

One person was unable to hide his wounds in his work environment, which led him to resign:

Quit my job as a preschool-school age swimming teacher. Because I was too depressed and embarrassed of my cuts especially in front of little children who I am a role model to. (Male, 20)

The psychological toll of hiding the evidence of self-injury to maintain a facade of success and well-being was particularly well-articulated by one woman who wrote:

I felt detached from everyone around me, like I was fake and no one knew the real me. It was as though there was another side to me that no one knew and they could never know. On the outside I was picture perfect, or should have been, to them I had everything going for me. (Female, 19)

While it is understandable that participants hid their scars to prevent others from finding out about their self-injury, it is unclear from most of the responses what type of reactions these participants expected to receive. However, a few participants did specify that they hid their wounds or scars to avoid being judged:

Having to hide what I have done because it embarrasses me and I don't want anyone to see how pathetic I am. (Female, 33)

Having to deal with the scars that takes a very long time to heal, and having to hide them from others so I don't get judged. (Female, 19)

For one adolescent, being found out would have resulted in the loss of a position of responsibility: "I am a school leader, and one of the reasons I got in was because I 
had stopped self harming, and if it was found out that I did slip up my role would be taken from me"(Female, 16).

It is apparent from the majority of examples within this sub-theme that the anticipation, rather than the actual experience, of being judged for transgressing social norms through self-injuring drove participants to conceal the evidence of their self-injurious behaviours. Some participants, however, did report actually being judged by others for engaging in NSSI.

\subsubsection{Being judged by others for transgressions}

Despite the considerable effort undertaken by people to protect themselves from being found out as transgressors, at times the scars or wounds were seen by others. Some participants lied to avoid being judged, although this was not always sufficient:

Embarrassment when someone saw the wounds and I had to lie about them to avoid being judged negatively. (Female, 24)

Having to lie to people that are close to you, particularly the ones that know you self-injure. Often they won't believe you anyway and you feel like you have angered or disappointed them yet again. (Female, 19)

Indeed, several participants mentioned being judged by others as a negative consequence of their self-injury:

Made to feel even worse at ED by their judgements and treatment. (Female, 33)

The internet friend I told scolded me and got very upset about it. (Female, 16)

People telling me I overreacted (not understanding). (Female, 24)

My parents and friends were disappointed in me. (Female, 16)

One woman stated that her self-injury had resulted in her girlfriend leaving her and her family sending her to therapy, thus providing evidence that self-injurious behaviour within her social network is perceived as an unacceptable manifestation of psychopathology, which needs to be fixed.

Although people tended to report being judged for failing to live up to familial, peer, and societal expectations because they had self-injured, one woman 
described a situation where the opposite occurred and her self-injury was normalised:

Made it more likely that Student Health people might actually bloody well take seriously when I said I was depressed and needed help. Therefore made me feel ten million times more unloved when they still didn't. Apparently self-harm is a 'perfectly natural coping mechanism' and means nothing. What a load of bollocks. Did these people even go to med school? (Female, 18)

In this instance, she had tried to use self-injury - which she perceived as an abnormal coping mechanism - to signal her need for mental health support. Other participants' self-injurious behaviours were viewed as pathological, but her behaviour was dismissed as an acceptable coping strategy, leading her to feel rejected and frustrated.

\subsubsection{Transgressions cause others to suffer}

The effect of self-injury on family and friends was another sub-theme identified in the overarching theme of the self becoming a transgressor:

It upset the person closest to me to see me in that state. (Female, 19)

Hurting my husband by him knowing the extent of my emotional distress was so much that I hurt myself. (Female, 26)

I frightened those I love. (Female, 30)

My family and people found out and got really scared and hurt. I felt so guilty and even worse for making them feel that way. (Female, 16)

This may have been an emotional release to me, but it seems that it was an uncomfortable display to other people around me; this distressed them deeply. (Female, 20)

In one case, the participant's episode of self-injury actually led to the her boyfriend relapsing himself: "My boyfriend got upset with me self harming as he had issues with it also and we were trying to stop together and then he was upset and self harmed also" (Female, 18).

\subsubsection{Self becomes helper}

The second theme-self becomes a helper - was identified from the positive consequences reported by the participants. This theme comprised of ways in which participants had succeeded in helping themselves; in particular, three sub-themes of 
emotion regulation, accessing support/treatment, and the presence of physical wounds were identified.

\subsubsection{Regulating emotions}

The most common positive emotional experiences reported by participants following their most recent episode of NSSI were calmness, relief, and release:

Calming down, stilling my emotions which had been in turmoil. (Female, 26)

I gained some relief from the overwhelming feelings of despair I was experiencing. (Female, 52)

I felt a break from all the pain I was feeling. It was a huge release. Almost euphoric. (Female, 18)

Calm, more relaxed, almost a meditation. (Female, 35)

Felt calmer, more grounded and in control. (Female, 42)

By regulating their overwhelming emotions, people were able to move forward, whether this involved getting some sleep, going to work, or generally managing the situation they were in:

Felt calmer, released 'stuck' emotions so I could cry, sleep, soothe myself, care for myself. (Female, 24).

It worked to calm me down and get me out of the house and to work on time. (Female, 23)

I calmed down enough to finish my day without too much trouble or time spent stressing. (Female, 20)

I felt like I had released all the built up internal emotion and better able to cope with the situation without my feelings getting the best of me. (Female, 20)

I sat down and was able to get on with my study without feeling stressed or anxious. (Male, 18)

Some participants commented on the temporary nature of the relief noting that it was "momentary", "lasted for a few hours", or that they felt "better in the short term". However, for one person, the memory of self-injury continued to induce a calming effect: "It was a special secret that only I knew about. The memory creates a small feeling of calm" (Female, 27). 
Self-injury not only allowed people to reduce particular negative emotions, such as despair and anxiety, but also to induce positive emotions, such as pride, hope, and happiness:

Feeling stronger, feeling happier. (Female, 20)

Discover hope. (Female, 21)

I felt proud because I felt like injuring myself was a good way of dealing with my emotions because I could think of nothing else to do to make it better - as if I had found an effective solution. (Female, 17)

Furthermore, successfully regulating their emotions appeared to engender a sense of agency for people, allowing them to feel more in control. In this way, self-injury was an active solution to feeling emotionally overwhelmed: "I felt calmer, and that I had achieved something" (Female, 17).

Importantly, a few participants reported decreased suicidality following NSSI, which they evaluated as a positive consequence:

Overwhelming impulse to die was reduced. (Female, 42)

Decrease of suicidal thoughts. (Male, 20)

Easing of stress and the feeling of wanting to die. (Female, 21)

I didn't try and kill myself. (Female, 39)

It is worth noting that any reductions in suicidal ideation would have involved cognitive as well as emotion regulation, as one of the above responses explains.

Aside from decreased suicidal thoughts, only a couple of participants identified positive cognitive consequences following their most recent episode of self-injury:

Able to think more clearly. (Female, 18)

Increased cognitive clarity. (Male, 32)

However, the lack of cognitive consequences reported may have resulted from the open-ended structure of the questions, particularly if emotional consequences are more salient. 


\subsubsection{Accessing support and/or treatment}

Several participants received help or support from others as a positive consequence of their self-injury:

My husband looked after me and was very gentle with me after I hurt myself. (Female, 23)

That my friend was made aware of how I was feeling and there for me. (Female, 20)

I had a discussion with my flatmate as he noticed the wounds a few days later - I discovered that he had been through similar things and we were able to talk about it openly - he has since been very supportive. (Female, 27)

People attended to me with more interest and put in genuine effort to help me. (Female, 20)

My boyfriend and I had a long talk about my inability to cope with stress and anger and self loathing. I agreed whenever I was feeling in too deep or like I wanted to hurt myself again that I would call him any time. (Female, 20)

For these participants, self-injury facilitated access to support by signalling the intensity of their distress to concerned friends and family members.

Some people were prompted to actively seek treatment or support from others following their self-injury:

I eventually got help from a counsellor. (Female, 19)

I spoke to my counsellor who referred me to mental health services after hurting myself 3 times in a month. (Female, 28)

Allowed me to realise just how badly I was distressed, meaning I went to ask for help from others. (Female, 27)

Finding a great new GP who described the process going on and who identified what I had experienced ever since I can remember. (Female, 35)

Even when actively seeking support from friends proved to be ineffective for one woman, she felt compelled to continue to search for the support she needed:

Realised how serious the situation was, that I absolutely didn't have the strength to deal with this by myself. Had already asked for help from friends/ex-boyfriend, but now realised that since they wouldn't help, MUST get help by other means. (Female, 18)

For her, this realisation was a positive consequence of her self-injury episode.

Two people reported being hospitalised as a positive consequence of their self-injury with one participant stating: “Got a bed in psych unit quicker and 
therefore felt safer and cared for" (Female, 41). Another woman went back on mood stabilisers as a result of her self-injury and also began taking sleeping pills, which she found helpful.

Although help-seeking typically resulted in some form of attention from others, only three people specifically identified receiving attention or sympathy from others as a positive consequence of self-injury:

Attention from others. (Female, 19)

Opportunity for attention. (Female, 18)

Felt like now that I have a deepish cut, people who may happen to see it -even though I try to hide it- will feel sympathy for me and like me more. (Female, 19)

The last response is particularly intriguing as this woman's expectation of how people will react to her cut is antithetical to the responses given by other participants within the self becomes transgressor theme. However, at the same time, she acknowledges trying to hide the injury, implying that she is aware of the status of self-injury as a socially transgressive behaviour. It may be that people within this woman's community judge those who attempt to conceal self-inflicted wounds more favourably than those who display them openly.

\subsubsection{The physical wound}

Contrary to the negative evaluation of wounds or scarring in the self becomes transgressor theme, the physical evidence of self-injury within the self becomes helper theme was identified as a positive consequence. It is worth noting, however, that many more people evaluated wounds and scarring as a negative consequence than as a positive consequence.

It was not readily apparent from the quotes why people reported blood, scars, and bruises as positive consequences. It is likely that these physical manifestations of self-injury were interpreted positively for different reasons, as is evident from the following responses:

I had a large burn mark on my arm. It looked pretty flash, eh. (Male, 19)

I saw blood. (Female, 27) 
Needing to attend to the cut on my leg. Stop the bleeding, apply a plaster and anti-septic. (Female, 35)

Unfortunately, there is not enough detail provided in these responses to determine why participants experienced the sight of their blood or wounds as positive.

\subsection{Summary of the findings from the open-ended responses}

The open-ended responses highlight the important influence of negative social interactions (especially interpersonal conflict) and community norms on the incidence and maintenance of NSSI. The paradox inherent in self-injury functioning simultaneously as an act of transgression and an act of self-help was evident in the consequences reported by participants. Negotiating the dissonance that results from this paradox has the potential to maintain NSSI if people attempt to avoid, or escape from, the painful, self-referent emotions that occur following self-injury through further self-injurious behaviours.

\section{DISCUSSION}

In this study, I was primarily interested in examining three hypotheses informed by the EAM (Gratz et al., 2006), extant literature on why people engage in NSSI, and the results of the Interpretative Functional Analysis that I presented in the previous chapter. Specifically, I hypothesised that (1) affect regulation would be endorsed as the primary function of NSSI, (2) intrapersonal functions would be more highly endorsed than interpersonal functions, and (3) negative affect would decrease following self-injury but positive affect would increase. Finally, I was also interested in exploring whether people reported shifts in negative, self-referent thoughts following episodes of NSSI.

\subsection{Affect regulation and self-punishment are the primary functions of NSSI}

Although the average ratings and mean ranks for affect regulation were slightly higher than self-punishment for participants' global and most recent episodes of NSSI, these functions had the same median and were not significantly different from one another. However, for both global and most recent episodes, 
affect regulation and self-punishment were rated significantly higher than any of the other functions. This is consistent with Klonsky and Glenn's (2009) finding that affect regulation and self-punishment respectively were the first and second most commonly reported functions of NSSI among university students.

As I discussed in Chapter 3, it is likely that punishing oneself through selfinjury is a specific form of affect regulation because in such instances the selfinjurious act is carried out to regulate self-directed anger and self-hatred (Chapman et al., 2006; Klonsky, 2007, 2009). Other functions (i.e., marking distress, antidissociation/feeling generation, and anti-suicide) that could conceivably be incorporated within a broader conceptualisation of affect regulation than that which is represented in the ISAS (Klonsky \& Glenn, 2009) were also highly endorsed by the participants in this study. Although these three functions differed significantly to affect regulation, self-punishment, and all the other subscales, they did not differ significantly from one another.

The five most highly endorsed functions across both the global and most recent episodes of NSSI - affect regulation, self-punishment, marking distress, antidissociation/feeling generation, and anti-suicide - can all be understood as forms of experiential avoidance, thus supporting the EAM's premise, which is that self-injury primarily functions as an experientially avoidant behaviour (Chapman et al., 2006). Although people did endorse access functions (e.g., interpersonal influence, revenge, peer-bonding) of self-injury along with the avoidant functions, these were rated significantly lower.

Furthermore, the role of self-injury in facilitating affect regulation was exemplified in the open-ended responses from participants about the consequences of their self-injury. Most notably, self-injury functioned to calm participants down and to help them relax. However, it was also evident that transgressing social norms through self-injury led participants to feel ashamed, guilty, and fearful of being judged by others. These responses provide some insight into how the cycle of selfinjury may be maintained through self-punishment. 
Given that self-injury functions as a way to avoid or escape negative affect, painful self-referential emotions, such as shame and guilt, may be particularly powerful antecedents for future NSSI. The experience of these emotions could lead people to conclude that they deserve to be punished for their transgressive behaviour, which in turn may result in further self-injury. Indeed, women with BPD who demonstrated greater non-verbal shame behaviours were more likely to selfinjure than women who did not show these behaviours (Brown, Linehan, Comtois, Murray, \& Chapman, 2009).

\subsection{Intrapersonal functions are more highly endorsed than interpersonal functions}

It is apparent from the ISAS results that people endorsed items from across all of the single-function subscales. This range of responses calls into question the clinical utility and validity of single-function models and emphasises the importance of multi-function conceptualisations of NSSI, such as the EAM (Chapman et al., 2006) and the FFM (Nock \& Prinstein, 2004, 2005).

For participants' global and most recent episodes of NSSI, the most appropriate solution in both cases comprised of two clusters, which was consistent with previous theoretical and empirical demarcations between intrapersonal and interpersonal motivations for self-injury (Klonsky \& Glenn, 2009; Nock \& Prinstein, 2004). The only anomaly in the cluster solution for participants' general episodes was the inclusion of self-care within the interpersonal cluster when theoretically it should have clustered with intrapersonal functions.

On inspection, the self-care items demonstrated good face validity (e.g., "creating a physical injury that is easier to care for than my emotional distress") making it unlikely that these items actually reflect interpersonal motivations for selfinjury. In Klonsky and Glenn's (2009) study, self-care also was more closely aligned with interpersonal, rather than intrapersonal, functions, but the loadings of this subscale on the intrapersonal and interpersonal factors were very similar. In the current study, self-care was the last function from the interpersonal group to enter the solution, which is comparable to Klonsky and Glenn's (2009) self-care factor 
loadings. The status of self-care as a liminal function is supported by the cluster solution that was identified for participants' most recent episode. In this analysis, self-care moved from the interpersonal into the intrapersonal cluster.

Comparisons of the intrapersonal versus interpersonal functions demonstrated that participants were significantly more likely to report self-injuring for intrapersonal than interpersonal reasons, in relation to both a single episode of self-injury and their global episodes of NSSI. Indeed, only nine people endorsed interpersonal functions more highly than intrapersonal functions. This is consistent with the EAM's (Gratz et al., 2006) exclusion of interpersonal reasons as primary to the maintenance of NSSI.

Considering that difficulties in interpersonal relationships were the most commonly reported events to occur prior to participants' most recent episode of selfinjury, it is noteworthy that self-injuring for interpersonal functions was infrequently endorsed. One possibility for this incongruence is that people who self-injure may blame themselves for interpersonal conflict, leading them to consider themselves unworthy of the support or care of others. As such, they may be more likely to selfinjure as a form of punishment rather than as a way to access support from others.

\subsection{Negative affect decreased and positive affect increased following NSSI}

Self-reported negative affect, hostility, sadness, and fear all decreased significantly following self-injury, but there were no significant changes in guilt. This is surprising in light of my qualitative analysis where I proposed that people become transgressors because of their self-injurious behaviour, which would seem to suggest that participants' self-reported guilt should increase following NSSI. Individual ranks show that 71 people experienced a decrease in guilt following self-injury but 62 people experienced an increase. It may be that the qualitative findings are predominantly reflecting the latter group of participants.

Although there was no significant difference in participants' ratings of general positive affect before and after the episode, there was a significant increase in joviality (e.g., happy, joyful, energetic). Furthermore, while not identified by 
Watson and Clark (1994) as a positive emotion, serenity (i.e., calm, relaxed, at ease) also increased significantly. More specifically, three quarters of the participants reported experiencing increased serenity after they self-injured. This aligns with the positive, open-ended consequences described by participants as one of the most frequently recalled emotion words within the self becomes helper theme was calm.

The effectiveness of self-injury as an emotion regulation strategy is supported by other research (e.g., Klonsky, 2007) and forms the basis of the EAM (Chapman et al., 2006). However, it is important to keep in mind that not all participants managed to regulate their emotions through self-injury; indeed, several participants reported feeling worse following their most recent episode. However, it should be noted that participants were not asked to refer to a specific timeframe when reporting the emotions that followed their episode of NSSI. As a result, participants who endorsed improved affect may have been reflecting on how they felt immediately after selfinjuring, whereas participants who endorsed worse affect may have been focusing on a more temporally distant time period (i.e., days after having self-injured).

\subsection{Negative cognitions decrease following NSSI}

Studying shifts in cognitions following self-injury is a recent development in the NSSI literature (see Nock et al., 2009), but research suggests that attempts to regulate unwanted thoughts may play an important role in the maintenance of selfinjury (Najmi et al., 2007). In the current study, negative automatic thoughts reportedly decreased significantly following self-injury, while positive/neutral thoughts increased significantly. However, caution is required when interpreting these results given that the ATQ-R (Kendall et al., 1989) has not been validated for use in reference to a particular episode of behaviour. The high internal consistency scores obtained in the current study for this measure, however, provide some reassurance that the scale is a reliable measure of self-referent thoughts in this context.

Similarly to the reports of affect changes following self-injury, there was variability in the number of participants who experienced shifts in cognitions. Some 
people reported that their negative cognitions increased following NSSI, while others experienced no change in their negative thoughts. A substantial number of people also reported that their positive/neutral cognitions did not change after they self-injured. Although cognitive regulation is minimised within the EAM (Chapman et al., 2006), experiential avoidance theory more generally (cf., Hayes et al., 1996) does not privilege emotion escape/avoidance over cognitive escape/avoidance.

It is unclear from this study whether aversive emotions are triggered by unwanted thoughts or whether these thoughts occur in the context of aversive emotions. It is likely a combination of both, although some people's experience of self-injury may be primarily driven by either cognitive or emotional avoidance. For example, someone with OCD may self-injure specifically as a strategy to cope with intrusive thoughts. Furthermore, the relative absence of cognitive consequences in the open-ended survey responses suggests that self-injury is primarily used for emotion, rather than cognitive, regulation, or that emotional consequences are much more salient and therefore easier to recall.

\subsection{Strengths}

Given that it is somewhat atypical for phenomenological studies of self-injury to include previously validated and reliable measures, one of the strengths of this study was the measures chosen to assess the frequency, functions, and emotional antecedents and consequences of NSSI. Additionally, comparing the behavioural trajectories of participants' general and most recent episodes of self-injury provided further insight into the functional complexity of individual episodes of NSSI. Another strength of the current study and a novel approach to testing whether selfinjury functions primarily as a form of experiential avoidance was examining whether the content of participants' automatic thoughts shifted following self-injury. Finally, the diversity of the sample was also a strength as much of the self-injury research with community populations has focused exclusively on university students. Although the majority of participants in this study were at university, approximately $40 \%$ were engaged in other occupations. 


\subsection{Limitations}

The data collected in this study focused on historical episodes of self-injury and therefore is subject to limitations associated with retrospective self-report (Tourangeau, 2000). By restricting inclusion to people who had self-injured within the past 12 months, I attempted to minimise the impact of retrospection on the data. However, the average amount of time that had lapsed between participants' most recent episode of self-injury and their participation in the survey was 81 days and this length of time varied considerably between participants. Sophisticated ecological momentary assessment studies are needed to examine the functions of NSSI in more depth without retrospective bias.

Another limitation of this study was that I was unable to explicitly assess the role of positive or negative reinforcement in participants' episodes of self-injury because the ISAS does not categorise the function subscales according to reinforcement value. However, it is possible to deduce from the items whether they reflect escape/avoidance or access motivations. For example, the affect regulation subscale contains items about reducing aversive emotion and it is therefore likely that people who endorse these items engage in NSSI to escape or avoid emotional experiences.

\subsection{Conclusion}

The behavioural trajectory of self-injury described in EAM (Chapman et al., 2006) is broadly consistent with the results of this study, which suggests that the EAM can be usefully applied to New Zealanders' experiences of self-injury. While this model is not representative of every episode of self-injury, experiential avoidance did appear to be the primary function of NSSI in this sample. Given that self-injury is commonly used to escape or avoid negative emotions and/or thoughts, in the next chapter I focus on assessing whether people who self-injure can be differentiated from people who do not hurt themselves on purpose on the basis of their general intrapersonal experiences and coping styles. 


\section{CHAPTER SIX}

\section{Comparing the intrapersonal experiences and coping styles of people with and without a history of NSSI}

\section{INTRODUCTION}

In the interview and survey studies I presented in Chapters 4 and 5, I focused on clarifying the antecedents and consequences of NSSI episodes to determine whether self-injury functions primarily as a form of experiential avoidance within Aotearoa New Zealand. In the current chapter, I take a step back from the immediate behavioural contingencies of self-injury to compare the global intrapersonal experiences (i.e., thoughts and emotions) and coping styles of people who have selfinjured with those who have never self-injured.

Research on the emotional and cognitive experiences and coping styles of people who self-injure complements functional analyses of NSSI in a number of ways. First, a wealth of studies support the affect-regulation function of NSSI (Klonsky, 2007), making it important to understand more about the general affective experiences of people who self-injure. Given that the complex relationship between affect and cognition is difficult to disentangle (Hayes et al., 1996), it is equally important to consider whether specific types of thoughts differentiate people with a history of NSSI from those without such a history.

Indeed, experiencing negative thoughts and feelings about oneself has been found to predict engagement in NSSI among university students (Armey \& Crowther, 2008), while the temperament dimension of negative affectivity was shown to predict NSSI among community-based adolescents (Baetens et al., 2011). People with a history of NSSI have also been shown to experience higher levels of negative emotion than people who have never hurt themselves on purpose (Andover et al., 2007; Brown et al., 2007). These findings are useful because they advance our understanding of the broader emotional and cognitive context in which individual episodes of NSSI are situated. 
Second, although NSSI is frequently referred to as a coping strategy in the literature (e.g., Claes \& Vandereycken, 2007; Nock, 2010; Swenson et al., 2008) and functional analyses highlight that self-injury is an adaptive process for managing distress, the taxonomic parameters of this coping response and its relationship to other ways of coping are seldom addressed. One of the few exceptions is the EAM (Chapman et al., 2006) because it proposes that self-injury is an avoidant coping strategy, which belongs in a functional response class with other avoidant behaviours. Support for this contention comes from studies showing that people with a history of NSSI use more avoidant coping strategies to deal with stressful events than people who have never self-injured (Andover et al., 2007; Brown et al., 2007). Furthermore, the use of avoidant coping by people who have self-injured has been shown to vary as a function of self-injury severity (Hasking et al., 2008).

Given that the intrapersonal experiences of people who self-injure appear to be more negative in general than people who do not self-injure (Brown et al., 2007; Hasking et al., 2008), it seems hardly surprising that they are more likely to utilise avoidant coping strategies (including self-injury) to escape from their distressing emotions and thoughts. Such an assertion, however, assumes a causal direction from negative affect and cognitions to self-injury. It is possible, of course, that self-injury causes negative affect and cognitions. In one of the few prospective studies of NSSI, Hankin and Abela (2011) identified that having a more negative cognitive style predicted future self-injury among adolescents. However, this research is an exception as most of the studies examining the relationships between global intrapersonal experiences, avoidant coping strategies, and NSSI have been crosssectional, which precludes causal inferences in either direction.

Although additional over-time studies are necessary to determine whether negative affect and cognitions cause NSSI, research on the mechanisms underlying NSSI is also sorely needed. To this end, researchers have begun to investigate whether people with a history of NSSI have a general tendency towards avoidant coping. Certainly, it makes intuitive sense that a propensity towards avoidance may 
underlie people's continued use of NSSI and evidence in support of this hypothesis would add weight to the contention that self-injury functions as an avoidant coping strategy.

A tendency towards thought suppression, the process of attempting to prevent oneself from thinking about certain topics (Wegner \& Zanakos, 1994), is a specific form of experiential avoidance that has been implicated as a causal mechanism underlying NSSI (Najmi et al., 2007). In one cross-sectional study, the relationship between adolescents' self-reported reactivity to emotions and the frequency of NSSI was mediated by thought suppression (Najmi et al., 2007); in another study, the relationship between past experience of childhood sexual abuse and NSSI frequency was mediated by avoidance symptoms of PTSD (Weierich \& Nock, 2008). While these findings suggest that the desire to avoid unwanted thoughts may play a key role in perpetuating self-injurious behaviours, these results need to be replicated longitudinally to confirm the hypothesised causal pathways (Najmi et al., 2007; Weierich \& Nock, 2008).

In the current study, I attempted to expand on my previous findings - that NSSI functions as both emotional and cognitive avoidance-by examining how people who have self-injured think, feel, and cope, compared with people who have never self-injured. More specifically, I hypothesised that people with a history of self-injury would report experiencing higher levels of general negative affect (Brown et al., 2007; Hasking et al., 2008), more negative automatic thoughts (Hankin \& Abela, 2011), and have a greater tendency towards avoidant coping (Andover et al., 2007; Brown et al., 2007) than people without a history of self-injury. If people do use NSSI as a strategy to avoid experiencing negative emotions and thoughts, it seems probable that they would report higher levels of general negative affect and aversive thoughts than people who do not self-injure.

Furthermore, in light of the dearth of over-time studies, I chose to survey participants about their affect, cognitions, and coping styles at two time-points, two months apart, to test whether negative affect and cognitions would predict new 
incidents of NSSI. Certainly, the EAM (Chapman et al., 2006) and previous empirical findings (Hankin \& Abela, 2011) suggest that negative intrapersonal experiences should place people at risk of engaging in future episodes of self-injury.

Consequently, I hypothesised that negative intrapersonal experiences reported at Time 1 (T1) would be causally related to new episodes of NSSI reported at Time 2 (T2). Finally, if a predictive relationship between negative intrapersonal experiences and NSSI was identified, I hypothesised that this relationship would be mediated by a tendency towards experiential avoidance (Najmi et al., 2007).

\section{METHOD}

\subsection{Participants}

A total of 443 (excluding repeat entries) first year Psychology students from Victoria University of Wellington consented to participate in the T1 phase of this study, but only 408 (92\%) surveys were retained for analysis (see Figure 9 for rationale). Almost three quarters of the participants were female (72.1\%); $113(27.7 \%)$ were male, and $1(0.2 \%)$ was male-to-female transsexual. The average age of participants was 19.46 years $(\mathrm{SD}=3.43)$, with a range of 17 to 44 years. Although the majority of participants identified as New Zealand European (83.3\%), a variety of ethnicities were reported: 34 (8.3\%) Māori, 14 (3.4\%) Chinese, 9 (2.2\%) Indian, 7 (1.7\%) Samoan, 4 (1.0\%) Cook Islands Maori, and 43 (10.5\%) other (e.g., South African, Japanese).

One or more mental health diagnoses were reported by $67(16.4 \%)$ of the participants, with an average of $1.46(\mathrm{SD}=0.75)$ diagnoses per person (range: $1-5)$. Out of the participants who reported receiving a mental health diagnosis in their lifetimes, 58 (87.9\%) had been diagnosed with Depression, 24 (36.4\%) with Anxiety, 6 (9.1\%) with Anorexia, 4 (6.1\%) with BPD, 3 (4.5\%) with Bulimia, and 1 (1.5\%) each with Bipolar Disorder, Substance Use Disorder and Drug-induced Psychosis. More than one third of the participants $(37.5 \%)$ had received therapy or counselling in the past, but only 19 (4.7\%) were currently engaged in therapy or counselling. 


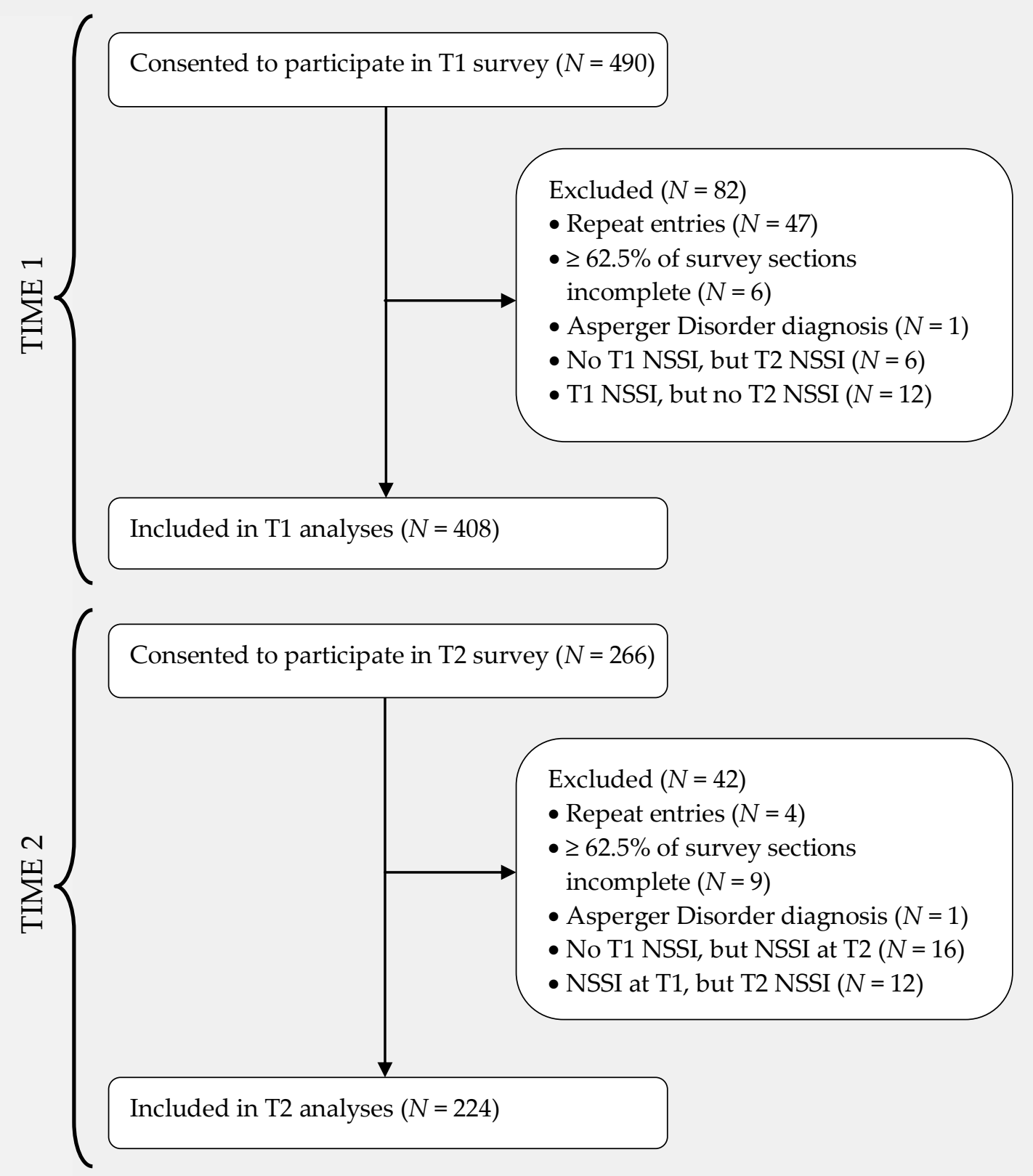

Figure 9. Flow of participants through the study at Times 1 and 2.

Out of the 262 (excluding repeat entries) surveys completed at T2, 224 were retained for analysis (see Figure 9 for rationale). Prior to deleting cases, the response rate for T2 was 59.1\%. Chi-square tests of independence at an alpha level of .05 were used to determine whether there were any significant differences between the participants who only completed the T1 survey and those who completed both surveys. Although participants who completed the T1, but not the T2 survey, were 
significantly more like to be male ${ }^{28}\left(\mathrm{X}^{2}(1, N=407)=17.30, \mathrm{p}<.001\right)$, they were just as likely to report a history of mental illness $\left(\mathrm{X}^{2}(1, N=405)=1.65, \mathrm{p}=.20\right)$ and/or selfinjury $\left(\mathrm{X}^{2}(1, N=408)=.107, \mathrm{p}=.74\right)$.

\subsection{Procedure}

First year Psychology students at Victoria University of Wellington are required to participate in four hours of research each trimester to fulfill their course requirements. The current study was listed on the Psychology Department's Experimetrix.com website, where students are presented with a range of study choices. Students who do not want to take part in research studies are able to summarise published journal articles instead. Prospective participants were advised prior to sign-up that participation would involve completing two online surveys, both of which included questions about NSSI. Students who had a history of NSSI and those who had never self-injured were invited to participate. Once they had signed up, they were required to click "yes" at the end of the information page to indicate that they consented to participate in the study before they were directed through to the first survey. Those who did not consent to participate were directed to a page that contained the following message: "You did not consent to participate in this survey. Please close your browser window to exit".

The study was conducted across two time-points: (1) participants completed an online survey (see Appendix K) via Surveymonkey and (2) two months later, they were emailed a link to the same survey (excluding the demographic questions) and invited to complete it a second time. Each participant was given two weeks to complete the second survey ${ }^{29}$ and was sent up to two reminders. Surveys were matched using the student ID numbers that participants provided at each time point. The two-month time period between survey completions was chosen for pragmatic reasons to ensure that the study could be completed within one university trimester.

\footnotetext{
28 The one transsexual student was omitted from this analysis.

${ }^{29}$ One student completed the second survey three weeks after the initial reminder was sent. This survey was included in the analyses.
} 
As with the online survey described in the previous chapter, anyone who started either of the surveys for the current study was emailed a copy of the contact details for various support organisations (see Appendix L). This was done to ensure that any participants who did not finish the survey would still have access to relevant debriefing information. The options for support were also presented on the final pages of both the T1 and T2 surveys. Finally, it was suggested to participants on the survey information page that they ask a support person to be available while they complete the survey if appropriate. Each student received half an hour of research credits for completing the T1 survey and a \$10 Motor Trade Association voucher for completing the T2 survey. The study ${ }^{30}$ was approved by the Multiregion Ethics Committee, a New Zealand Health and Disability Ethics Committee administered by the Ministry of Health.

\subsection{Measures}

\subsubsection{The Deliberate Self-harm Inventory}

The modified version of the DSHI (Gratz, 2001) used in the second study to measure the prevalence and frequency of 14 different types of NSSI was also used in the current study. These modifications are described in detail in Chapter 5 (see p. 179) and the psychometric properties of this measure are described in Chapter 4 (see p. 101).

\subsubsection{The Acceptance and Action Questionnaire}

The Acceptance and Action Questionnaire (AAQ; Hayes et al., 2004) is a 9item, self-report questionnaire designed to measure experiential avoidance. Each item (e.g., "If I could magically remove all the painful experiences I've had in my life, I would do so") is rated on a seven point likert scale from 1 (never true) to 7 (always true); four items are reverse scored. Responses are summed to give a maximum score

\footnotetext{
${ }^{30}$ I intended to complete an ecological momentary assessment study, but a change in ethics policy at Victoria University meant that the ethics committee could no longer consider my application. As a result of the significant delays I experienced following this policy change, I was unable to follow through with my original study and had to amend my research design.
} 
of 63 with higher scores indicating a stronger tendency towards experiential avoidance.

The AAQ has demonstrated adequate internal consistency $(\alpha=.70)$ and testretest reliability $(r=.65)$; fairly low test-retest reliability is to be expected given that experiential avoidance is conceptualised as a behavioural process, which in all likelihood is amenable to contextual influences (Hayes et al., 2004). Convergent validity with thought suppression and psychopathology measures has also been established (Hayes et al., 2004). The only time this measure has been used in a selfinjury study, the authors did not report any study-specific psychometric statistics (Chapman, Specht, \& Cellucci, 2005).

\subsubsection{The Positive and Negative Affect Schedule}

The PANAS-X (Watson \& Clark, 1994) is a 60-item, self-report measure that contains the following 13 scales: negative affect, positive affect, fear, hostility, guilt, sadness, joviality, self-assurance, attentiveness, shyness, fatigue, serenity, and surprise. The emotions included in each of these scales are listed in Chapter 5 (see p. 181). Each emotion word (e.g., angry, joyful) is rated on a five point scale from 1 (very slightly or not at all) to 5 (extremely). Mean scores are calculated for each of the 13 scales.

In the current study, the PANAS- $X$ was used to measure affect experienced by participants in general; accordingly, the general timeframe instructions were chosen (i.e., "Indicate to what extent you feel this way in general, that is, on the average"). As stated in Chapter 5, the excellent psychometric properties of the PANAS-X are well-established (Watson \& Clark, 1994). For example, internal consistency rates across six samples for the negative affect scale when completed according to the general timeframe instructions ranged from .85 to .93 (Watson \& Clark, 1994).

\subsubsection{The White Bear Suppression Inventory}

The White Bear Suppression Inventory (WBSI; Wegner \& Zanakos, 1994) is a 15-item, self-report measure of people's propensity to suppress unwanted thoughts. Each item (e.g., "There are things that I try not to think about") is rated from 1 
(strongly disagree) to 5 (strongly agree). Responses are summed to give a maximum score of 75; higher scores indicate a greater tendency towards thought suppression. The WBSI has demonstrated good internal consistency $(\alpha=.85-.89)$ across a number of studies (Blumberg, 2000; Rassin, 2003; Wegner \& Zanakos, 1994). Scores on the WBSI have been found to converge with scores on well-validated depression and anxiety measures (Wegner \& Zanakos, 1994), and NSSI frequency (Chapman et al., 2005; Najmi et al., 2007).

\subsubsection{The Depression Anxiety Stress Scales}

The short version of the Depression Anxiety Stress Scales (DASS-21; Lovibond \& Lovibond, 1995) is a 21-item, self-report measure developed to test the occurrence of three connected constructs - depression, anxiety, and tension/stress - within the past week. Each construct is operationalised in a 7-item subscale; item examples include: "I felt that I had nothing to look forward to" (depression), "I felt scared without any good reason" (anxiety), and "I found it hard to wind down" (tension/stress). Items are rated on a four point scale from 0 (did not apply to me at all) to 3 (applied to me very much, or most of the time). Scores are summed to give a maximum of 21 for each subscale and 63 for the total score. Cronbach alphas for the DASS-21 subscales range from .82 to .94 , which suggest it is a highly reliable measure (Antony, Bieling, Cox, Enns, \& Swinson, 1998; Henry \& Crawford, 2005). It has also demonstrated adequate construct validity (Henry \& Crawford, 2005).

\subsubsection{The Automatic Thoughts Questionnaire - short version}

Netemeyer and colleagues (2002) constructed a 15-item version of the original 30-item Automatic Thoughts Questionnaire (ATQ; Hollon \& Kendall, 1980) by factor-analysing the ATQ and inspecting the content of individual items. Although the psychometric properties of the shortened version have not been extensively tested, it demonstrated excellent internal consistencies $(\alpha=.92-.96)$ across three samples (Netemeyer et al., 2002). The frequencies of specific, negative automatic thoughts (e.g., "I'm no good", "It's just not worth it") experienced within the past week are rated on a five point scale from 1 (not at all) to 5 (all the time). Scores are 
summed to total a maximum of 75 , with higher scores signifying more pervasive negative thoughts.

\subsubsection{The Brief COPE}

The Brief COPE was developed by Carver (1997) to reduce the participant response burden associated with the 60-item COPE inventory (Carver, Scheier, \& Weintraub, 1989). Coping styles, rather than situational responses, were assessed in the current study and, as such, the instructions and items were presented in a dispositional format (Carver et al., 1989). All but two of the COPE scales are present in the Brief COPE, but there are only two, rather than four, items per scale. Carver (1997) also added a new two item scale, self-blame, to the Brief COPE.

In total, the Brief COPE is comprised of the following 14 scales: selfdistraction (e.g., "I turn to work or other activities to take my mind off things"), active coping (e.g., "I take action to try to make the situation better"), denial (e.g., "I refuse to believe that it has happened"), substance use (e.g., "I use alcohol or other drugs to make myself feel better"), use of emotional support (e.g., "I get emotional support from others"), use of instrumental support (e.g., "I get help and advice from other people"), behavioural disengagement (e.g., "I give up trying to deal with it"), venting (e.g., "I express my negative feelings”), positive reframing ( e.g., "I look for something good in what is happening"), planning (e.g., "I think hard about what steps to take"), humour (e.g., "I make jokes about it"), acceptance (e.g., "I learn to live with it"), religion (e.g., "I pray or meditate"), and self-blame (e.g., "I blame myself for things that happened").

Each item is rated on a four point scale from 1 (I usually don't do this at all) to 4 (I usually do this a lot). Item responses are summed to give a maximum score of eight per scale. Averaged scale reliabilities have been found to vary considerably from .50 (venting) to .90 (substance use) but caution is required when interpreting internal consistency values in measures with only two items per scale (Carver, 1997). 


\section{RESULTS}

\subsection{Prevalence and frequencies of self-injury types}

The prevalence and frequencies of NSSI types for both time-points are presented in Table 12. At T1, almost half the participants (46.8\%) reported having engaged in NSSI in their lifetimes. The average age of onset for NSSI was 13.17 years $(\mathrm{SD}=2.84$; range: $3-19)$, with cutting $(24.8 \%)$, severe scratching $(21.8 \%)$, and sticking sharp objects into the skin (19.9\%) the most commonly reported types of self-injury. The least common types of NSSI were rubbing sandpaper on the body $(2.7 \%)$, dripping acid onto the skin $(2.0 \%)$, and using bleach or oven cleaner to scrub the skin (0.2\%). No participants reported breaking their own bones. Additionally, 16 (3.9\%) participants endorsed engaging in at least one other type of NSSI that was not listed in the DSHI (e.g., "rubbed steel wool against skin", "scalded self with boiling water"). The 191 participants who reported self-injuring had engaged in an average of $3.12(S D=2.26)$ types of NSSI, with a range of 1 to 13 .

Despite the high lifetime prevalence of self-injury at T1, only $56(32.0 \%)$ of the participants who endorsed a history of NSSI reported having injured themselves on purpose within the past 12 months. In the two months prior to T1 survey completion, a total of 42 participants had injured themselves on average 7.26 times $(S D=17.76$; range: 1-100). A more detailed breakdown of self-injury recency for both time points is presented in Table 13.

As is to be expected given the prevalence of NSSI at T1, almost half (47.8\%) of the participants endorsed a lifetime history of NSSI at T2. The most frequently endorsed types of NSSI at T2 were the same as T1: cutting (29.5\%), severe scratching (20.5\%), and sticking sharp objects into the skin (20.5\%). The least frequently endorsed types of NSSI at T2 were also the same as T1: rubbing sandpaper on the body $(2.2 \%)$, dripping acid (2.2\%) onto the skin, and using bleach or oven cleaner $(0.9 \%)$ to scrub the skin. Once again, no participants reported breaking bones. Comparable to T1 reports of NSSI, the participants who reported a history of NSSI at 
Table 12

Frequencies of NSSI types reported at Times 1 and 2

\begin{tabular}{|c|c|c|c|c|c|c|c|c|c|c|c|c|}
\hline \multirow{3}{*}{ NSSI type } & \multicolumn{6}{|c|}{ Time $1(N=408)$} & \multicolumn{6}{|c|}{ Time $2(N=224)$} \\
\hline & \multirow{2}{*}{$\begin{array}{l}\text { N (\%) } \\
\text { endorsing } \\
\text { NSSI type }\end{array}$} & \multicolumn{5}{|c|}{ Frequency of NSSI types } & \multirow{2}{*}{$\begin{array}{l}\text { N (\%) } \\
\text { endorsing } \\
\text { NSSI type }\end{array}$} & \multicolumn{5}{|c|}{ Frequency of NSSI types } \\
\hline & & Never & 1 time & 2-10 times & $\begin{array}{l}11-50 \\
\text { times }\end{array}$ & $\begin{array}{l}50 \\
\text { times }\end{array}$ & & Never & 1 time & $\begin{array}{l}2-10 \\
\text { times }\end{array}$ & $\begin{array}{l}11-50 \\
\text { times }\end{array}$ & $\begin{array}{l}>50 \\
\text { times }\end{array}$ \\
\hline $\begin{array}{l}\text { Cutting wrists, arms, or } \\
\text { other areas of body }\end{array}$ & $101(24.8)$ & $307(75.2)$ & $14(3.4)$ & $50(12.3)$ & $25(6.1)$ & $11(2.7)$ & $66(29.5)$ & $157(70.1)$ & $8(3.6)$ & $34(15.2)$ & $15(6.7)$ & $6(2.7)$ \\
\hline $\begin{array}{l}\text { Severe scratching to extent } \\
\text { of bleeding/ scarring }\end{array}$ & $89(21.8)$ & $315(77.2)$ & $16(3.9)$ & $44(10.8)$ & $20(4.9)$ & $5(1.2)$ & $46(20.5)$ & $172(76.8)$ & $4(1.8)$ & $26(11.6)$ & $8(3.6)$ & $3(1.3)$ \\
\hline $\begin{array}{l}\text { Sticking sharp objects into } \\
\text { skin }\end{array}$ & $81(19.9)$ & $325(79.7)$ & $12(2.9)$ & $54(13.2)$ & $9(2.2)$ & $0(0.0)$ & $46(20.5)$ & $175(78.1)$ & $2(0.9)$ & $33(14.7)$ & $5(2.2)$ & $0(0.0)$ \\
\hline $\begin{array}{l}\text { Carving words/pictures/ } \\
\text { designs/marks into skin }\end{array}$ & $70(17.2)$ & $337(82.6)$ & $33(8.1)$ & $30(7.4)$ & $3(0.7)$ & $1(0.2)$ & $44(19.6)$ & $180(80.4)$ & $16(7.1)$ & $23(10.3)$ & $1(0.4)$ & $0(0.0)$ \\
\hline $\begin{array}{l}\text { Burning with cigarette/ } \\
\text { lighter/match }\end{array}$ & $54(13.2)$ & $350(85.8)$ & $15(3.7)$ & $33(8.1)$ & $5(1.2)$ & $0(0.0)$ & $31(13.8)$ & $192(85.7)$ & $6(2.7)$ & $22(9.8)$ & $0(0.0)$ & $0(0.0)$ \\
\hline $\begin{array}{l}\text { Preventing wounds from } \\
\text { healing }\end{array}$ & $50(12.3)$ & $355(87.0)$ & $2(0.5)$ & $23(5.6)$ & $15(3.7)$ & $8(2.0)$ & $34(15.2)$ & $189(84.4)$ & $4(1.8)$ & $14(6.3)$ & $4(1.8)$ & $6(2.7)$ \\
\hline $\begin{array}{l}\text { Banging head to extent of } \\
\text { bruising }\end{array}$ & $50(12.3)$ & $357(87.5)$ & $10(2.5)$ & $27(6.6)$ & $7(1.7)$ & $1(0.2)$ & $23(10.3)$ & $195(87.1)$ & $1(0.4)$ & $16(7.1)$ & $1(0.4)$ & $1(0.4)$ \\
\hline $\begin{array}{l}\text { Punching to extent of } \\
\text { bruising }\end{array}$ & $40(9.8)$ & $367(90.0)$ & $9(2.2)$ & $28(6.9)$ & $1(0.2)$ & $0(0.0)$ & $19(8.5)$ & $203(90.6)$ & $1(0.4)$ & $11(4.9)$ & $3(1.3)$ & $0(0.0)$ \\
\hline $\begin{array}{l}\text { Biting to extent of breaking } \\
\text { skin }\end{array}$ & $24(5.9)$ & $384(94.1)$ & $6(1.5)$ & $15(3.7)$ & $3(0.7)$ & $0(0.0)$ & $16(7.1)$ & $201(89.7)$ & $5(2.2)$ & $8(3.6)$ & $1(0.4)$ & $1(0.4)$ \\
\hline Rubbing glass into skin & $16(3.9)$ & $386(94.6)$ & $6(1.5)$ & $9(2.2)$ & $1(0.2)$ & $0(0.0)$ & $7(3.1)$ & $212(94.6)$ & $2(0.9)$ & $4(1.8)$ & $0(0.0)$ & $0(0.0)$ \\
\hline Rubbing sandpaper on body & $11(2.7)$ & $396(97.1)$ & $9(2.2)$ & $2(0.5)$ & $0(0.0)$ & $0(0.0)$ & $5(2.2)$ & $216(96.4)$ & $4(1.8)$ & $0(0.0)$ & $0(0.0)$ & $0(0.0)$ \\
\hline Dripping acid onto skin & $8(2.0)$ & $395(96.8)$ & $3(0.7)$ & $5(1.2)$ & $0(0.0)$ & $0(0.0)$ & $5(2.2)$ & $214(95.5)$ & $4(1.8)$ & $1(0.4)$ & $0(0.0)$ & $0(0.0)$ \\
\hline $\begin{array}{l}\text { Using bleach/oven cleaner to } \\
\text { scrub skin }\end{array}$ & $1(0.2)$ & $406(99.5)$ & $1(0.2)$ & $0(0.0)$ & $0(0.0)$ & $0(0.0)$ & $2(0.9)$ & $221(98.7)$ & $0(0.0)$ & $1(0.4)$ & $0(0.0)$ & $0(0.0)$ \\
\hline Breaking own bones & $0(0.0)$ & $0(0.0)$ & $0(0.0)$ & $0(0.0)$ & $0(0.0)$ & $0(0.0)$ & $0(0.0)$ & $0(0.0)$ & $0(0.0)$ & $0(0.0)$ & $0(0.0)$ & $0(0.0)$ \\
\hline
\end{tabular}

Note. N endorsing NSSI type may not equal frequencies of engagement as some participants endorsed type of NSSI but did not report frequency. Frequencies may not add up to $100 \%$ because of missing data. 
$\mathrm{T} 2$ had engaged in an average of $3.27(\mathrm{SD}=2.27)$ types of self-injury with a range of 1 to 10 .

Table 13

NSSI recency at Times 1 and 2

\begin{tabular}{lcc}
\hline \multirow{2}{*}{ Recency of last NSSI episode } & Time $\mathbf{1}(\mathbf{N}=\mathbf{1 7 2})$ & Time $\mathbf{2}(\mathbf{N}=\mathbf{1 0 6})$ \\
\cline { 2 - 3 } & $\boldsymbol{N}(\mathbf{\%})$ & $\boldsymbol{N}(\mathbf{\%})$ \\
\hline$<1$ week & $13(3.2)$ & $7(6.6)$ \\
1 week $>1$ month & $11(2.7)$ & $6(5.7)$ \\
1 month $>$ 6 months & $21(5.1)$ & $16(15.1)$ \\
6 months $>1$ year & $11(2.7)$ & $16(15.1)$ \\
1 year $>2$ years & $18(15.1)$ & $9(14.8)$ \\
2 years $>3$ years & $19(16.0)$ & $10(16.4)$ \\
3 years $>4$ years & $26(21.8)$ & $12(19.7)$ \\
4 year $>5$ years & $15(12.6)$ & $10(16.4)$ \\
$>5$ years & $38(31.9)$ & $20(32.8)$ \\
\hline
\end{tabular}

The order of frequency for the different types of NSSI was identical at T2 to T1, with one exception; burning oneself with a cigarette, lighter, or match was more frequently endorsed than preventing wounds from healing at T1 than T2. At T2, other forms of NSSI (e.g., "hit myself on the head with a rock', "flagellation”) were endorsed by $9(4.0 \%)$ participants. A total of 23 people reported self-injuring on average 6.43 times $(\mathrm{SD}=12.72$; range: $1-60)$ in the two months between completing the T1 and T2 surveys. Out of the 23 people who reported self-injuring between T1 and T2, a total of 11 also reported self-injuring in the two months prior to T1.

\subsection{Do people who have self-injured experience more negative emotions and thoughts?}

To examine whether people who have self-injured generally experience higher levels of negative emotions and thoughts than people who have never selfinjured, I planned to conduct a multivariate analysis of variance (MANOVA) using the T1 negative affect and emotion (i.e., fear, hostility, guilt, and sadness) scores 
from the PANAS-X, the T1 depression, anxiety, and stress scores from the DASS-21, and the T1 ATQ-R total scores. All of these scales demonstrated good to excellent internal consistency ( $\alpha=.82-.96)$ as is evident in Table 14 .

Prior to running the MANOVA, visual inspection of the histograms and Q-Q plots for these nine variables showed that their distributions were positively skewed with varying degrees of kurtosis (see Table 14). Kolmogorov-Smirnov tests with Lilliefors Correction (Field, 2009) confirmed that the distributions for all of the variables for both groups were significantly non-normal, $D^{\prime} s(185-217) \geq .10$ (all p's < .001). To test for univariate outliers, I converted all of the scores for these nine variables into $z$-scores to check whether any were less than -3.29 or greater than 3.29 as recommended by Tabachnik and Fidell (2007). In total, seven scores ${ }^{31}$ from different participants were identified as possible outliers. Removing these scores did not alter the significance of subsequent normality tests and, as a result, they were retained in the analyses.

Despite the significantly non-normal distributions of these and other variables analysed for this chapter, I chose to continue with MANOVA tests for the following reasons. First, the sample size was large enough to reduce the impact of skewness and kurtosis on the analyses (Tabachnick \& Fidell, 2007). Although positive and negative kurtosis can lead to underestimates of variance, this typically only applies to samples that consist of less than 100 or 200 cases respectively (Tabachnick \& Fidell, 2007; Waternaux, 1976). Second, MANOVAs are thought to be robust to deviations from normality when Pillai's trace is used (Olson, 1974). Transforming the data was considered unnecessary given these guidelines and undesirable since transformation alters the constructs under investigation (Grayson, 2004). Finally, the most pragmatic reason to use MANOVA is simply that there are no commonly used non-parametric alternatives (Field, 2009).

\footnotetext{
31 Two of the scores were from the ATQ and DASS anxiety scales, while three scores were from the DASS stress scale.
} 
Table 14

Time 1 reliability coefficients, descriptive statistics, and ANOVAs for the No NSSI versus Lifetime NSSI groups

\begin{tabular}{|c|c|c|c|c|c|c|c|c|c|}
\hline \multirow[b]{2}{*}{ Variable } & \multirow[b]{2}{*}{ Cronbach's $\alpha$} & \multicolumn{3}{|c|}{ No history of NSSI ${ }^{1}$} & \multicolumn{3}{|c|}{ Lifetime history of NSSI ${ }^{2}$} & \multicolumn{2}{|c|}{ ANOVAs } \\
\hline & & $\mathrm{M}(\mathrm{SD})$ & Skewness & Kurtosis & $\mathrm{M}(\mathrm{SD})$ & Skewness & Kurtosis & $F$ & $\eta_{p^{2}}$ \\
\hline \multicolumn{10}{|l|}{ PANAS-X } \\
\hline Negative affect & .89 & $2.00(0.61)$ & 0.51 & -0.45 & $2.39(0.72)$ & 0.52 & -0.40 & $35.40^{*}$ & .08 \\
\hline Fear & .86 & $1.97(0.67)$ & 0.54 & -0.51 & $2.27(0.76)$ & 0.65 & -0.26 & $17.80^{*}$ & .04 \\
\hline Hostility & .83 & $1.87(0.62)$ & 0.74 & 0.21 & $2.25(0.72)$ & 0.47 & -0.40 & $32.00^{*}$ & .08 \\
\hline Guilt & .91 & $1.78(0.73)$ & 0.83 & -0.09 & $2.32(0.96)$ & 0.78 & -0.10 & $37.66^{*}$ & .09 \\
\hline Sadness & .90 & $2.09(0.79)$ & 0.73 & -0.22 & $2.57(0.95)$ & 0.38 & -0.70 & $29.50^{*}$ & .07 \\
\hline$A T Q-R$ & .96 & $22.70(9.61)$ & 1.94 & 4.20 & $28.70(13.78)$ & 1.28 & 0.79 & $22.05^{*}$ & .05 \\
\hline \multicolumn{10}{|l|}{ DASS-21 } \\
\hline Depression & .89 & $3.16(3.43)$ & 1.44 & 1.80 & $5.28(4.73)$ & 0.95 & -0.06 & $23.72^{*}$ & .06 \\
\hline Anxiety & .82 & $3.21(3.23)$ & 1.22 & 1.09 & $4.68(4.21)$ & 1.01 & 0.16 & $13.79^{*}$ & .04 \\
\hline Stress & .85 & $4.74(3.70)$ & 0.70 & -0.16 & $6.68(4.66)$ & 0.77 & -0.13 & $18.71^{*}$ & .05 \\
\hline WBSI & .92 & $45.60(11.92)$ & -0.35 & -0.18 & 51.59 (11.61) & -0.45 & 0.05 & $15.14^{*}$ & .04 \\
\hline$A A Q$ & .73 & $32.92(7.26)$ & 0.18 & -0.29 & 35.65 (7.44) & 0.28 & -0.22 & $29.30^{*}$ & .07 \\
\hline \multicolumn{10}{|l|}{ COPE } \\
\hline Self-distraction & .57 & $4.88(1.43)$ & 0.21 & -0.14 & $5.25(1.60)$ & 0.03 & -0.73 & 5.35 & .01 \\
\hline Denial & .69 & $2.71(1.10)$ & 1.69 & 2.53 & $2.67(1.14)$ & 1.98 & 6.57 & 0.10 & .00 \\
\hline Substance use & .91 & $2.79(1.30)$ & 1.85 & 3.33 & $3.49(1.90)$ & 1.16 & 0.27 & $19.97^{*}$ & .05 \\
\hline $\begin{array}{l}\text { Behavioural } \\
\text { disengagement }\end{array}$ & .70 & $2.71(1.03)$ & 1.44 & 1.33 & $3.13(1.33)$ & 1.27 & 1.16 & $13.33^{*}$ & .03 \\
\hline
\end{tabular}

Note. ${ }^{1} N^{\prime}$ s range from 213 to $217,{ }^{2} N$ 's range from 185 to 191 due to missing values.

${ }^{*} \mathrm{p}<.001$

All $d f^{\prime} s=1,384-406$. 
Non-parametric bivariate correlations revealed that significant positive relationships existed between all of the variables under investigation. Given that multicollinearity can reduce the power of MANOVA tests, Tabachnick and Fidell (2007) recommend carefully considering the inclusion of any variables that are correlated at or above .70, and excluding those at or above .90 . As to be expected, the highest correlations $\left(r_{\mathrm{s}}=.78-.90\right.$, all p's $\left.\geq .001\right)$ were observed between the negative affect and four negative emotion scales (fear, hostility, sadness, and guilt) from the PANAS-X. To avoid multicollinearity, the negative affect scale was excluded from the MANOVA; instead, the impact of self-injury history on global negative affect was tested using a one-way independent analysis of variance (ANOVA).

A MANOVA ${ }^{32}$ using Pillai's trace showed a significant effect of NSSI history on the intensity and/or frequency of negative emotions and thoughts experienced by participants, $V=0.11, F(8,377)=5.58, \mathrm{p}<.001$, partial eta squared $=.11$. The possibility of Type I error was inflated for this and all other MANOVAs presented in this chapter because the covariance matrices were significantly different (Box's $\mathrm{M}=$ 124.74, $F(36,472353)=3.39, \mathrm{p}<.001)$. However, Box's M needs to be interpreted with caution given that it is a sensitive test (Tabachnik \& Fidell, 2007) and Type I error is less likely if the test statistic is still significant at a reduced alpha of .001.

Follow-up ANOVAs ${ }^{33}$, using a Bonferroni corrected alpha of .006 (Field, 2009), showed that participants who had self-injured reported significantly higher levels of fear, hostility, guilt, sadness, depression, anxiety, and stress, as well as significantly more negative automatic thoughts than participants who had never self-injured (see Table 13). An ANOVA conducted to test whether global negative affect varied as a function of self-injury history also showed a significant effect, $F(1,406)=35.40, \mathrm{p}<$ .001 , partial eta squared $=.08$, with participants who had self-injured reporting higher negative affect.

\footnotetext{
32 Removing three multivariate outliers ( $\mathrm{p}$-values of Mahalanobis $\mathrm{D}^{2}<.001$ ) increased the value of the F-statistic to 6.09. Since the test was significant regardless of whether these cases were included or not, they were retained in the analysis.

${ }^{33}$ Non-parametric Kruskal-Wallis tests yielded comparable significance values to the ANOVAs.
} 
Given that the extent and/or intensity of negative emotions and thoughts varied significantly as a function of self-injury history, further analyses were carried out to determine whether recency of self-injurious behaviours would impact on these differences. To achieve this, participants with a history of self-injury were divided into two groups on the basis of whether or not they reported self-injuring in the previous 12 months. This resulted in a total of three groups for the subsequent analyses: No NSSI, Lifetime NSSI (i.e., had self-injured but not in the past 12 months), and 12-month NSSI (i.e., had self-injured in the past 12 months).

Exploratory analyses (i.e., histograms and Q-Q plots) showed that although all of the variables in the three groups exhibited skewness and kurtosis, not all of these distributions were significantly non-normal. As presented in Table 14, Kolmogorov-Smirnov tests with Lilliefors correction indicated that the distributions for all the variables in the No NSSI and Lifetime NSSI groups were significantly nonnormal $\left(D^{\prime} s(115-217) \geq .10, p^{\prime} s<.001\right)$, but that only the ATQR and DASS subscales in the 12-month NSSI group were significantly non-normal $D^{\prime} s(54-56) \geq .15$, p's $\leq .002$.

Multivariate tests ${ }^{34}$ using Pillai's trace showed that there was a significant effect of recency of NSSI on the intensity of negative emotions and thoughts experienced by participants, $V=.19, F(16,724)=4.63, \mathrm{p}<.001$, partial eta squared $=$ .09. The covariance matrices were significantly different (Box's $M=175.55, F(72$, $76924)=2.33, \mathrm{p}<.001)$. Follow-up ANOVAs ${ }^{35}$ with Bonferroni corrected alpha of .006 demonstrated that all of the variables of interest varied significantly as a function of NSSI recency (see Table 14). Furthermore, negative affect was also found to vary significantly as a function of NSSI recency, $F(2,389)=22.62, \mathrm{p}<.001$, partial eta squared $=.10$.

\footnotetext{
${ }^{34}$ Removing three multivariate outliers ( $\mathrm{p}$-values of Mahalanobis $\mathrm{D}^{2}<.001$ ) increased the value of the $F$-statistic to 4.91. Since the test was significant regardless of whether these cases were included or not, they were retained in the analysis.

${ }^{35}$ Kruskal-Wallis tests yielded comparable significance values to the ANOVAs.
} 
Table 14

Time 1 descriptive statistics and ANOVAs for the No NSSI, Lifetime NSSI, and 12-month NSSI groups

\begin{tabular}{|c|c|c|c|c|c|c|c|c|c|c|c|}
\hline \multirow[b]{2}{*}{ Variable } & \multicolumn{3}{|c|}{ No NSSI $^{1}$ a } & \multicolumn{3}{|c|}{ Lifetime NSSI $^{2 b}$} & \multicolumn{3}{|c|}{ 12-month NSSI ${ }^{3 \mathrm{c}}$} & \multicolumn{2}{|c|}{ ANOVAs } \\
\hline & M (SD) & Skewness & Kurtosis & M (SD) & Skewness & Kurtosis & M (SD) & Skewness & Kurtosis & $F$ & $\eta_{p^{2}}$ \\
\hline \multicolumn{12}{|l|}{ PANAS-X } \\
\hline Negative affect & $2.00(0.61)^{b, c}$ & 0.51 & -0.45 & $2.26(0.65)^{a, c}$ & 0.68 & -0.01 & $2.63(0.79)^{a, b}$ & 0.12 & -0.90 & $22.62^{*}$ & .10 \\
\hline Fear & $1.97(0.67)^{\mathrm{c}}$ & 0.54 & -0.51 & $2.15(0.70)^{\mathrm{c}}$ & 0.94 & 0.59 & $2.50(0.85)^{a, b}$ & 0.22 & -0.79 & $13.53^{*}$ & .07 \\
\hline Hostility & $1.87(0.62)^{b, c}$ & 0.74 & 0.21 & $2.13(0.70)^{a, c}$ & 0.89 & 0.46 & $2.46(0.69)^{a, b}$ & -0.15 & -0.41 & $20.31^{*}$ & .10 \\
\hline Guilt & $1.78(0.73)^{b, c}$ & 0.83 & -0.09 & $2.12(0.87)^{a, c}$ & 1.05 & 0.80 & $2.77(1.04)^{a, b}$ & 0.13 & -0.96 & $30.34^{*}$ & .14 \\
\hline Sadness & $2.09(0.79)^{b, c}$ & 0.73 & -0.22 & $2.40(0.90)^{a, c}$ & 0.78 & -0.03 & $2.98(0.93)^{a, b}$ & -0.38 & -0.47 & $26.87^{*}$ & .13 \\
\hline$A T Q-R$ & $22.70(9.61)^{c}$ & 1.94 & 4.20 & $25.91(11.78)^{c}$ & 1.66 & 2.79 & $35.44(15.21)^{a, b}$ & 0.74 & -0.75 & $26.19^{*}$ & .13 \\
\hline \multicolumn{12}{|l|}{ DASS-21 } \\
\hline Depression & $3.16(3.43)^{\mathrm{c}}$ & 1.44 & 1.80 & $4.30(4.09)^{\mathrm{c}}$ & 1.31 & 1.38 & $7.57(5.07)^{a, b}$ & 0.33 & -1.21 & $28.31^{*}$ & .13 \\
\hline Anxiety & $3.21(3.23)^{c}$ & 1.22 & 1.09 & $4.10(3.93)^{\mathrm{c}}$ & 1.25 & 0.79 & $6.07(4.50)^{a, b}$ & 0.69 & -0.36 & $12.41^{*}$ & .06 \\
\hline Stress & $4.74(3.70)^{\mathrm{c}}$ & 0.70 & -0.16 & $5.88(4.31)^{c}$ & 0.69 & -0.46 & $8.28(4.67)^{a, b}$ & 0.80 & -0.11 & $15.25^{*}$ & .08 \\
\hline WBSI \# & $45.60(11.92)^{\mathrm{b}, \mathrm{c}}$ & -0.35 & -0.18 & $49.64(11.43)^{\mathrm{a}, \mathrm{c}}$ & -0.41 & 0.12 & $55.64(11.25)^{a, b}$ & -0.93 & 0.85 & $18.12^{*}$ & .09 \\
\hline$A A Q \#$ & $32.92(7.26)^{\mathrm{c}}$ & 0.18 & -0.29 & $34.13(6.91)^{\mathrm{c}}$ & 0.26 & -0.26 & $39.29(7.19)^{a, b}$ & 0.17 & -0.43 & $18.87^{*}$ & .09 \\
\hline \multicolumn{12}{|l|}{ COPE \# } \\
\hline Self-distraction & $4.88(1.43)^{\mathrm{c}}$ & 0.21 & -0.14 & $5.12(1.61)$ & 0.16 & -0.71 & $5.54(1.54)^{a}$ & -0.04 & -0.88 & 4.19 & .02 \\
\hline Denial & $2.71(1.10)$ & 1.69 & 2.53 & $2.61(1.13)$ & 2.15 & 4.27 & $2.75(1.18)$ & 1.82 & 3.17 & 0.36 & .00 \\
\hline Substance use & $2.79(1.30)^{c}$ & 1.85 & 3.33 & $3.14(1.65)^{c}$ & 1.43 & 1.26 & $3.98(2.00)^{a, b}$ & 0.87 & -0.33 & $14.07^{*}$ & .07 \\
\hline $\begin{array}{l}\text { Behavioural } \\
\text { disengagement }\end{array}$ & $2.71(1.03)^{\mathrm{c}}$ & 1.44 & 1.33 & $2.99(1.26)$ & 1.52 & 2.32 & $3.39(1.40)^{a}$ & 0.96 & 0.16 & $8.69^{*}$ & .04 \\
\hline
\end{tabular}

Note. ${ }^{1} N^{\prime}$ s range from $213-217,{ }^{2} N$ 's range from $115-119$ and ${ }^{3} N^{\prime}$ s range from $54-56$ due to missing values.

${ }^{*} \mathrm{p}<.001$

All df's $=2,368$ except \# df's $=2,379$.

$\mathrm{a}, \mathrm{b}, \mathrm{c}$ Means with superscripts are significantly different to other group means. 
Games-Howell post-hoc comparisons conducted at an alpha level of .05 revealed several significant differences in the intensity and/or presence of negative emotions and thoughts between groups. Participants who had self-injured in the past 12 months reported significantly higher mean levels of negative affect, hostility, guilt, and sadness than participants who had self-injured more than 12 months ago and those who had never self-injured. Participants who had self-injured more than 12 months ago in turn reported significantly higher mean levels of negative affect, hostility, guilt, and sadness than participants who had never self-injured.

Self-reported mean levels of stress, depression, anxiety, fear, and frequency of negative automatic thoughts were significantly greater for participants who had selfinjured in the past 12 months compared to those who had self-injured in their lifetimes or who had never engaged in NSSI. Participants who had self-injured more than 12 months ago did not differ significantly from participants who had never selfinjured on stress, depression, anxiety, fear, and frequency of negative automatic thoughts. In sum, the intensity of aversive emotions and frequency of negative thoughts experienced by participants varied significantly as a function of how recently they had self-injured.

\subsection{Do people who have self-injured have a greater tendency towards avoidant coping?}

The WBSI and AAQ were used in the current study to measure participants' propensities towards avoidant coping, along with four subscales from the Brief COPE (self-distraction, denial, substance use, and behavioural disengagement) that are thought to tap into avoidant coping (Chapman, Specht, \& Cellucci, 2005). Histograms and Q-Q plots for each of the variables were inspected prior to conducting the planned multivariate analyses. All of the frequency distributions, with the exception of the AAQ for participants who had never self-injured, were significantly non-normal, $\left.D^{\prime} s(190-217) \geq .07, \mathrm{p}^{\prime} \mathrm{s} \leq .008\right)$. After converting the scores for the six coping variables into z-scores, a total of 10 scores (five each from the COPE denial and behavioural disengagement scales) were identified as possible 
outliers because they were greater than 3.29. Since removing these cases did not alter the normality distributions, they were retained for subsequent analyses.

Prior to conducting a MANOVA, a series of non-parametric bivariate correlations were carried out to check for multicollinearity. Although there were significant, positive relationships between all six coping variables, the strongest correlation - between the WBSI and the AAQ $\left(r_{\mathrm{s}}=.58, \mathrm{p}=0.01\right)$ - was not high enough to warrant concern (Tabachnik \& Fidell, 2007).

Multivariate analyses of variance ${ }^{36}$ using Pillai's trace showed that participants' tendencies towards avoidant coping varied as a function of NSSI history, $V=.11, F(6,39)=8.26, \mathrm{p}<.001$, partial eta squared $=.11$. The covariance matrices were significantly different (Box's $M=111.50, F(21,564687)=5.22, p<.001)$. Follow-up ANOVAs ${ }^{37}$ with a Bonferroni corrected alpha of .008 indicated that participants who had self-injured reported significantly higher levels of thought suppression, experiential avoidance, substance use, and behavioural disengagement compared to participants who had never self-injured.

To examine whether avoidant coping varies as a function of NSSI recency, participants who reported a lifetime history of NSSI were once again split into two groups; those who had self-injured in the past 12 months and those who had selfinjured more than 12 months ago. This resulted in three levels of the group variable: No NSSI, Lifetime NSSI, and 12-month NSSI. All of the frequency distributions of variables at each level demonstrated some skewness and kurtosis. Normality tests confirmed that all but two of these distributions (the AAQ scores for No NSSI and 12-month NSSI) were significantly non-normal $\left(D^{\prime} s(56-217) \geq .07, \mathrm{p}^{\prime} \mathrm{s} \leq .03\right)$.

\footnotetext{
${ }^{36}$ Removing three multivariate outliers ( $\mathrm{p}$-values of Mahalanobis $\mathrm{D}^{2}<.001$ ) increased the value of the $F$ - statistic to 8.97. Since the test was significant regardless of whether these cases were included or not, they were retained in the analysis.

${ }^{37}$ Kruskall-Wallis tests resulted in comparable significance levels for the AAQ, WBSI, and substance use scale ( $p$ $<.001$ ), but significance levels for the behavioural disengagement scale decreased slightly to $p=.001$. Denial was still non-significant $(\mathrm{p}=.46)$ but self-distraction $(\mathrm{p}=.02)$ was significant at an alpha level of .05 .
} 
A MANOVA $^{38}$ using Pillai's trace showed a significant effect of recency of NSSI on avoidant coping by participants, $V=0.18, F(12,750)=6.10, \mathrm{p}<.001$, partial eta squared $=.09$. The covariance matrices were significantly different (Box's $M=$ 128.82, $F(42,95396)=2.97, \mathrm{p}<.001)$. Follow-up ANOVAs ${ }^{39}$ using a Bonferroni corrected alpha of .008 demonstrated the same pattern of results observed for the two-group comparison. The propensity towards thought suppression and experiential avoidance, as well as substance use and behavioural disengagement varied significantly as a function of NSSI recency (see Table 14). NSSI recency, however, did not have a significant effect on self-distraction or denial.

Post-hoc Games-Howell comparisons indicated that participants who had self-injured in the past 12 months reported significantly higher levels of thought suppression than those who had self-injured more than 12 months ago or those who had never self-injured. Participants who had self-injured more than 12 months ago also reported significantly higher levels of thought suppression than those who had never self-injured.

Self-reported experiential avoidance and using substances to cope was significantly higher for participants who had self-injured in the past 12 months, when compared to participants who had self-injured more than 12 months ago or those who had never self-injured. However, there were no significant differences in experiential avoidance or substance use for participants who had self-injured more than 12 months ago when compared to participants who had never self-injured.

Finally, participants who had self-injured in the past 12 months reported significantly higher behavioural disengagement scores than those who had never self-injured, but not those who had self-injured more than 12 months ago. Participants who had self-injured more than 12 months ago did not report

\footnotetext{
${ }^{38}$ Removing four multivariate outliers (p-values of Mahalanobis $\mathrm{D}^{2}<.001$ ) increased the value of the F-statistic to 6.51. Since the test was significant regardless of whether these cases were included or not, they were retained in the analysis.

${ }^{39}$ Kruskal-Wallis tests comparing the three groups yielded comparable significance levels to the ANOVAs with one exception; denial decreased from $\mathrm{p}=.70$ to $\mathrm{p}=.39$.
} 
significantly higher behavioural disengagement than participants who had never self-injured.

\subsection{Is NSSI predicted by negative emotions and thoughts?}

To determine whether negative emotions and thoughts predicted NSSI, the 224 participants who completed both the T1 and T2 surveys were divided into two groups: (1) participants $(N=201)$ who had not self-injured in the two months between T1 and T2 (No NSSI group), and (2) participants $(N=23)$ who had selfinjured in the two months between T1 and T2 (NSSI group). Two binary logistic regressions ${ }^{40}$ using the forced entry method were conducted to test whether negative emotions and thoughts at T1 predicted group membership at T2. These models are presented in Table 15.

Although both of the regression models were significant, none of the predictors added significant predictive value to the respective models. With only 23 participants reporting self-injury in the two months between survey completions, there was insufficient power to predict self-injury group membership. Consequently, both models correctly predicted group membership for the No NSSI group $100 \%$ of the time, but correctly predicted group membership for the NSSI group $0 \%$ of the time.

Given that the sample size of participants who had self-injured between survey completions was too small to detect meaningful differences through logistic regression, cross-lagged panel correlations using maximum likelihood estimates were tested in AMOS to determine the causal direction of the relationships between negative intrapersonal experiences at T1 and new episodes of self-injury reported at T2. By excluding possible third variable effects, cross-lagged panel correlations permit causal inferences from correlational data (Kenny, 1975).

\footnotetext{
${ }^{40}$ Participants were asked how many times they had self-injured in the previous two months, but given the extremely skewed frequency distribution (skewness $=11.35$ ), I chose to create and subsequently utilise a categorical variable of self-injury versus no self-injury in the analyses.
} 
Table 15

Two binary logistic regression models for NSSI versus No NSSI

\begin{tabular}{|c|c|c|c|c|}
\hline & \multirow[b]{2}{*}{ B (SE) } & \multicolumn{3}{|c|}{ 95\% CI for Odds Ratio } \\
\hline & & Lower & Odds Ratio & Upper \\
\hline \multicolumn{5}{|c|}{ Model 1: NSSI $(N=23)$ vs No NSSI $(N=194)$} \\
\hline \multicolumn{5}{|l|}{ Included } \\
\hline Constant & $-4.03(0.78)^{*}$ & & & \\
\hline Negative affect & $0.48(0.41)$ & 0.72 & 1.62 & 3.63 \\
\hline Automatic Thoughts & $0.03(0.02)$ & 0.99 & 1.03 & 1.07 \\
\hline \multicolumn{5}{|c|}{ Model 2: NSSI $(N=23)$ vs No NSSI $(N=201)$} \\
\hline \multicolumn{5}{|l|}{ Included } \\
\hline Constant & $-4.37(0.87)^{* *}$ & & & \\
\hline Fear & $0.15(0.43)$ & 0.51 & 1.16 & 2.68 \\
\hline Hostility & $0.36(0.45)$ & 0.59 & 1.44 & 3.47 \\
\hline Guilt & $0.52(0.37)$ & 0.81 & 1.68 & 3.48 \\
\hline Sadness & $-0.06(0.38)$ & 0.45 & 0.94 & 1.98 \\
\hline
\end{tabular}

Note. Model 1: $R^{2}=.20$ (Hosmer \& Lemeshow), .04 (Cox \& Snell), .09 (Nagelkerke). Model $X^{2}(2)=9.36, \mathrm{p}<.01 .{ }^{*} \mathrm{p}<.05$. Model 2: $R^{2}=.46$ (Hosmer \& Lemeshow), .05 (Cox \& Snell), .11 (Nagelkerke). Model $X^{2}(4)=11.95, \mathrm{p}<.05 .{ }^{* *} \mathrm{p}<.001$.

As is evident from Figures 10 to 15, all of the autoregressive paths were significant $(\mathrm{p}<.001)$ showing stability in negative emotions and thoughts over time (Martens \& Haase, 2006).

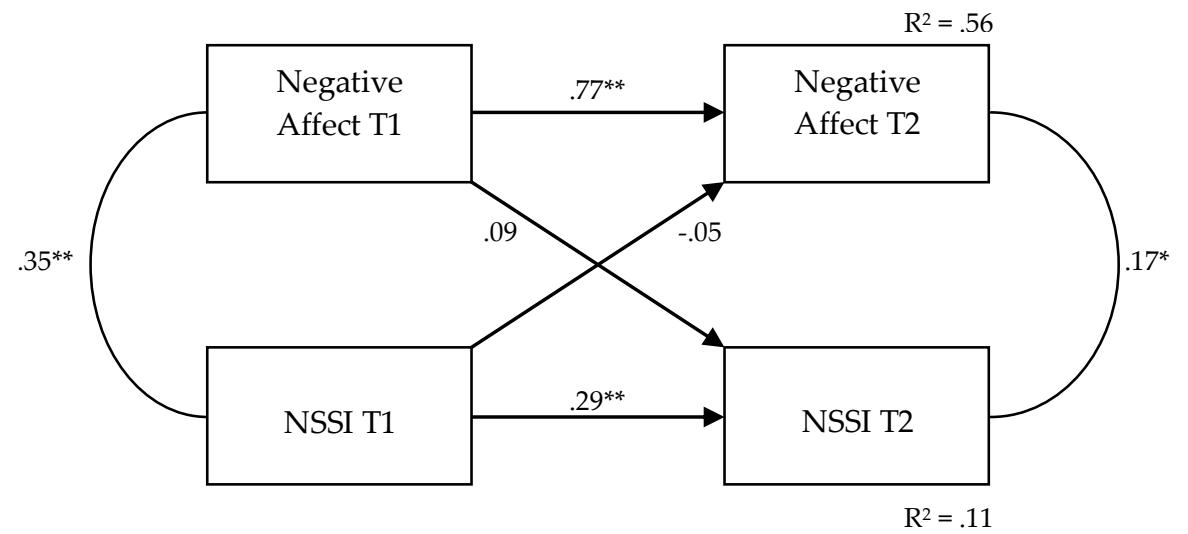

Note. ${ }^{*} \mathrm{p}<.05,{ }^{* *} \mathrm{p}<.001$

Figure 10. Cross-lagged panel model of NSSI and global negative affect. 


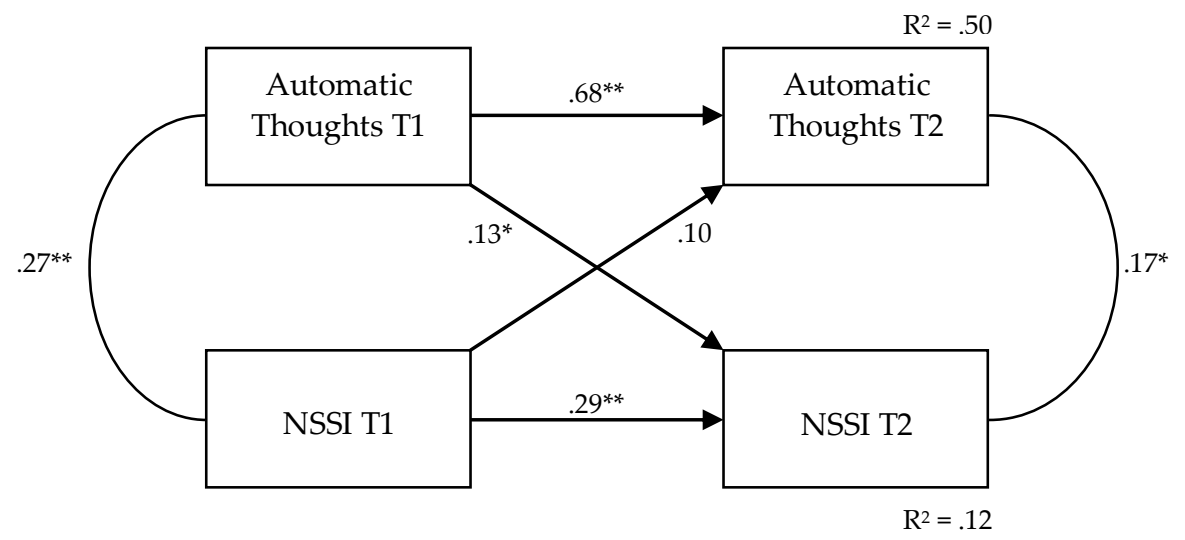

Note. ${ }^{*} \mathrm{p}<.05,{ }^{* *} \mathrm{p}<.001$

Figure 11. Cross-lagged panel model of NSSI and negative automatic thoughts.

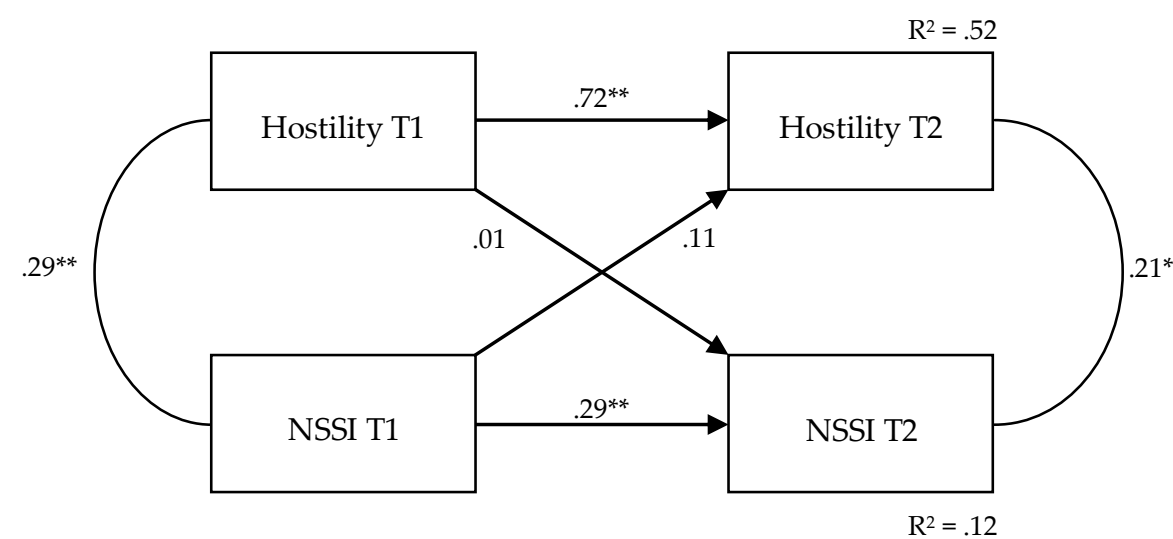

Note. ${ }^{*} \mathrm{p}<.01,{ }^{* *} \mathrm{p}<.001$

Figure 12. Cross-lagged panel model of NSSI and hostility.

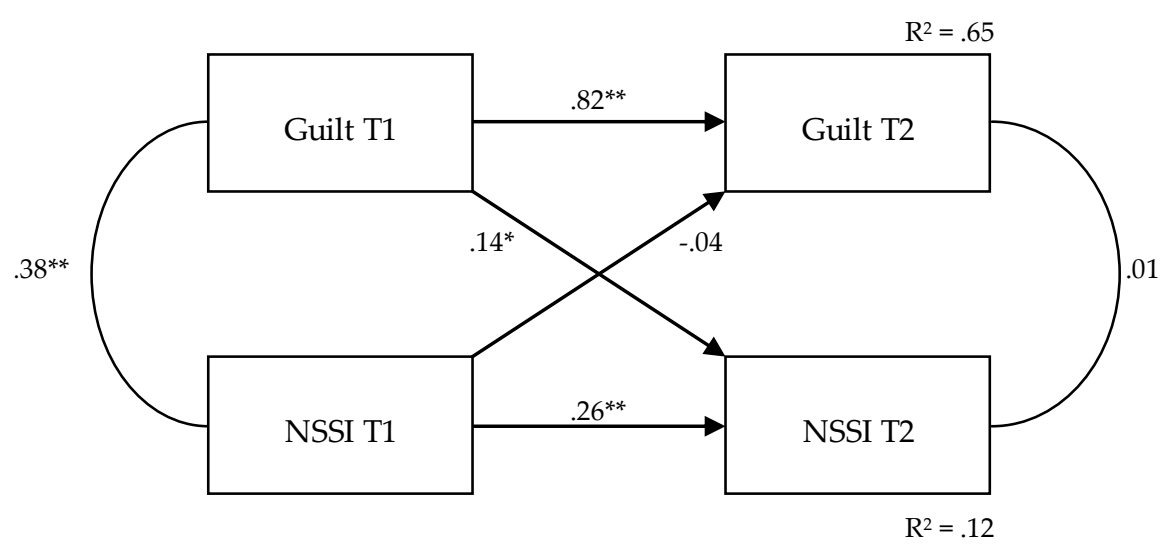

Note. ${ }^{*} \mathrm{p}<.05,{ }^{* *} \mathrm{p}<.001$

Figure 13. Cross-lagged panel model of NSSI and guilt. 


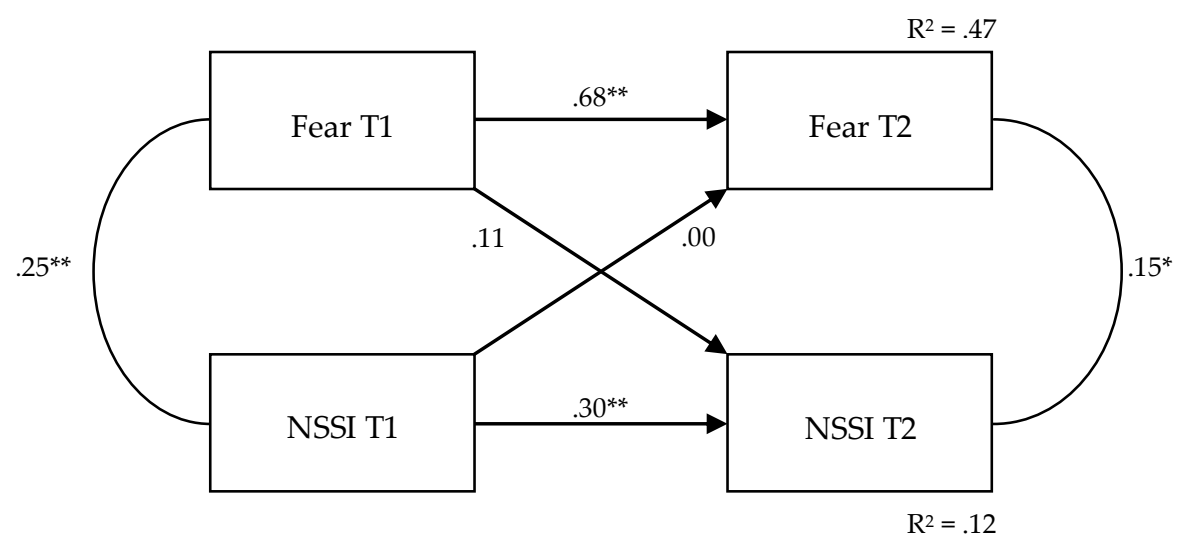

Note. ${ }^{*} \mathrm{p}<.05,{ }^{* *} \mathrm{p}<.001$

Figure 14. Cross-lagged panel model of NSSI and fear.

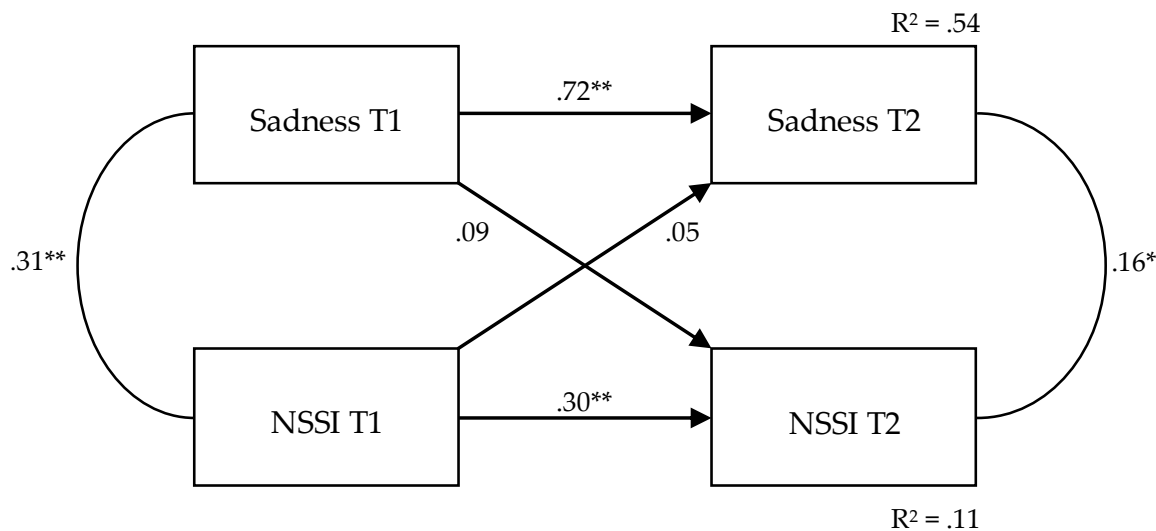

Note. ${ }^{*} \mathrm{p}<.05,{ }^{* *} \mathrm{p}<.001$

Figure 15. Cross-lagged panel model of NSSI and sadness.

Two of the cross-lag paths were significant at an alpha level of .05. The path from negative automatic thoughts at $\mathrm{T} 1$ to self-injury at $\mathrm{T} 2$ was significant $(\beta=.13, \mathrm{p}=$ .048), but the path from self-injury at T1 to negative automatic thoughts at T2 was non-significant $(\beta=.13, \mathrm{p}=.06)$. The path from guilt at T1 to self-injury at T2 was significant $(\beta=.14, \mathrm{p}=.03)$, but the path from self-injury at T1 to guilt at T2 was nonsignificant $(\beta=-.04, p=.31)$. These results suggest that dispositional automatic negative thoughts and guilt may be causal factors for later self-injury, but that selfinjury is not a causal factor for automatic negative thoughts and guilt. 


\subsection{Does avoidance mediate relationships between negative intrapersonal experiences and NSSI?}

The sample size of people who had self-injured between survey completions was too small to test mediation over time; instead, I used the T1 data to determine whether a dispositional tendency towards avoidance (i.e., experiential avoidance and thought suppression) mediated relationships between negative intrapersonal experiences (i.e., emotions and thoughts) and NSSI. Given that lifetime prevalence of NSSI was a dichotomous variable and standard mediation analyses are conducted with continuous variables, I used SPSS syntax ${ }^{41}$ written by Nathaniel Herr (www.nrhpsych.com/mediation/logmed.html) specifically for mediation analyses with dichotomous variables.

Mediation was determined using Baron and Kenny's (1986) criteria: mediation is observed if the predictor $(X)$ correlates with both the outcome $(Y)$ and the mediator $(\mathrm{M})$, and $\mathrm{M}$ then predicts $\mathrm{Y}$ while controlling for $\mathrm{X}$. Full mediation is observed when the relationship between $X$ and $Y$ while controlling for $M$ is reduced to zero (Baron \& Kenny, 1986). Partial mediation is observed when there is a reduction in the significance level of the relationship between $\mathrm{X}$ and $\mathrm{Y}$ when including M. Each mediation analysis was followed up with a Sobel test.

Experiential avoidance did not mediate the relationships between NSSI and negative affect (see Figure 16), fear (see Figure 18), hostility (see Figure 20), guilt (see Figure 22), sadness (see Figure 24), or negative automatic thoughts (see Figure 26). Thought suppression, however, was identified as a partial mediator in five models. As is evident from the figures, thought suppression partially mediated the relationships between NSSI and negative affect (see Figure 17), fear (see Figure 19), hostility (see Figure 21), sadness (see Figure 23), and automatic thoughts (see Figure 27). This suggests that specific forms of experiential avoidance (i.e., thought suppression) may underlie engagement in NSSI.

\footnotetext{
${ }^{41}$ Herr's syntax is based on equations presented by Mackinnon and Dwyer (1993).
} 


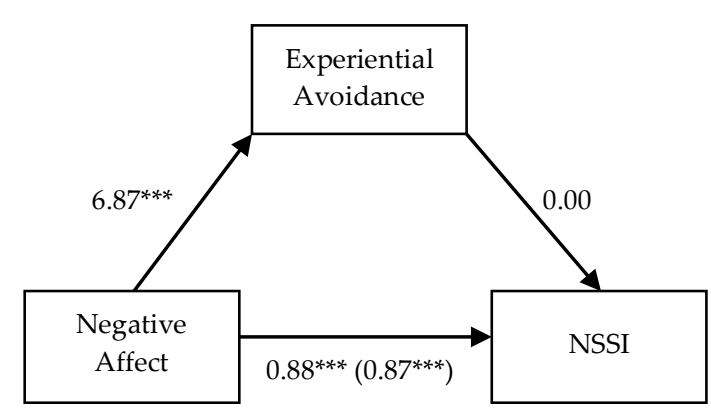

*** $\mathrm{p}<.001$

Figure 16. Unstandardised regression coefficients for the relationship between negative affect and NSSI which is not mediated by experiential avoidance. The unstandardised regression coefficient for the relationship between negative affect and NSSI controlling for experiential avoidance is in parentheses. Sobel's test $=0.11, \mathrm{SE}=0.07, \mathrm{p}=.91$.

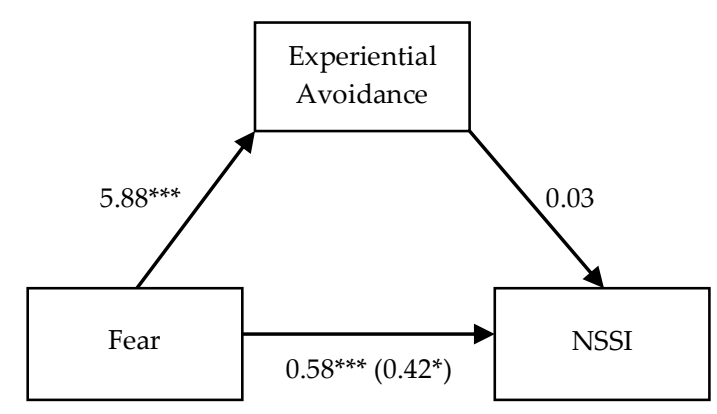

${ }^{*} \mathrm{p}<.05,{ }^{* * *} \mathrm{p}<.001$

Figure 18. Unstandardised regression coefficients for the relationship between fear and NSSI which is not mediated by experiential avoidance. The unstandardised regression coefficient for the relationship between fear and NSSI controlling for experiential avoidance is in parentheses. Sobel's test $=1.64, \mathrm{SE}=0.06, \mathrm{p}=.10$.

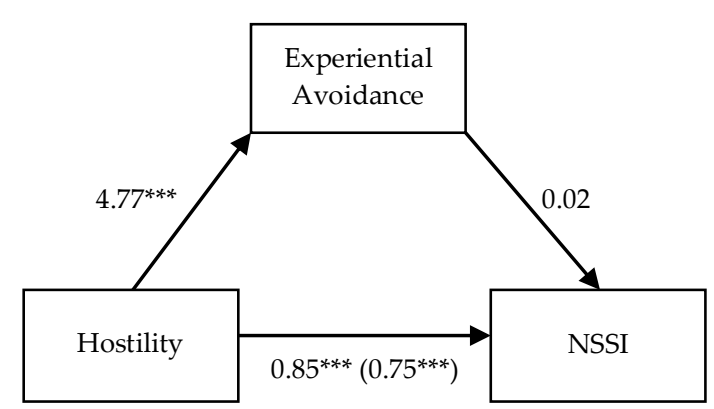

*** $\mathrm{p}<.001$

Figure 20. Unstandardised regression coefficients for the relationship between hostility and NSSI which is not mediated by experiential avoidance. The unstandardised regression coefficient for the relationship between hostility and NSSI controlling for experiential avoidance is in parentheses. Sobel's test $=1.36, \mathrm{SE}=0.06, \mathrm{p}=.17$.

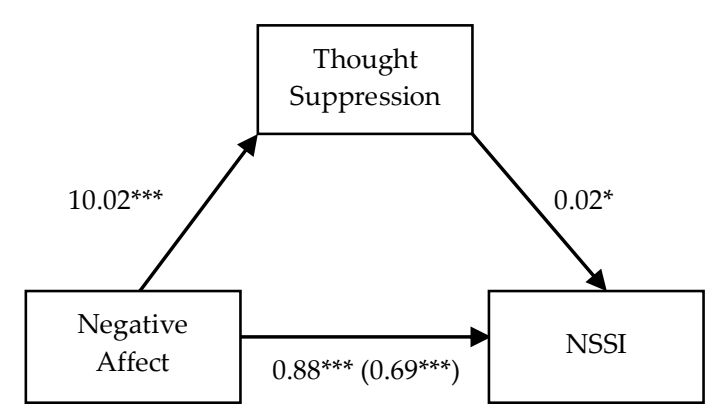

${ }^{*} \mathrm{p}<.05,{ }^{* *} \mathrm{p}<.001$

Figure 17. Unstandardised regression coefficients for the relationship between negative affect and NSSI which is partially mediated by thought suppression. The unstandardised regression coefficient for the relationship between negative affect and NSSI controlling for thought suppression is in parentheses. Sobel's test $=1.98, \mathrm{SE}=0.07, \mathrm{p}$ $=.048$.

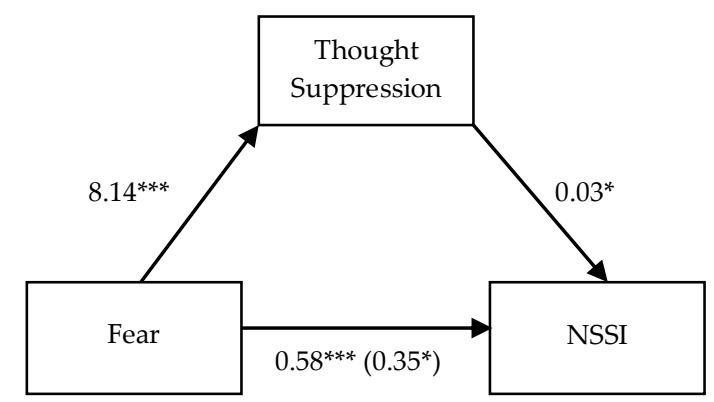

${ }^{*} \mathrm{p}<.05,{ }^{* *} \mathrm{p}<.01,{ }^{* * *} \mathrm{p}<.001$

Figure 19. Unstandardised regression coefficients for the relationship between fear and NSSI which is partially mediated by thought suppression. The unstandardised regression coefficient for the relationship between fear and NSSI controlling for thought suppression is in parentheses. Sobel's test $=3.17, \mathrm{SE}=0.06, \mathrm{p}=.002$.

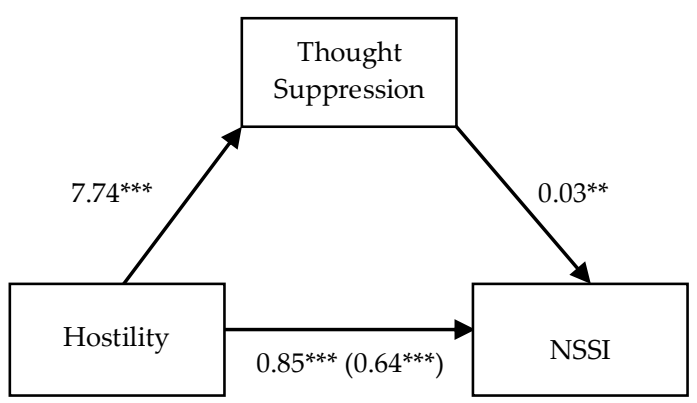

${ }^{* *} \mathrm{p}<.01,{ }^{* * *} \mathrm{p}<.001$

Figure 21. Unstandardised regression coefficients for the relationship between hostility and NSSI which is partially mediated by thought suppression. The unstandardised regression coefficient for the relationship between hostility and NSSI controlling for experiential avoidance is in parentheses. Sobel's test $=2.69, \mathrm{SE}=0.06, \mathrm{p}=.01$. 


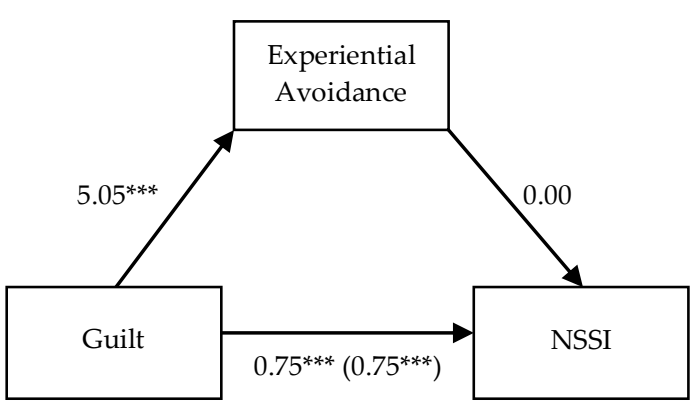

${ }^{* * *} \mathrm{p}<.001$

Figure 22. Unstandardised regression coefficients for the relationship between guilt and NSSI which is not mediated by experiential avoidance. The unstandardised regression coefficient for the relationship between guilt and NSSI controlling for experiential avoidance is in parentheses. Sobel's test $=0, \mathrm{SE}=0.06, \mathrm{p}=1$.

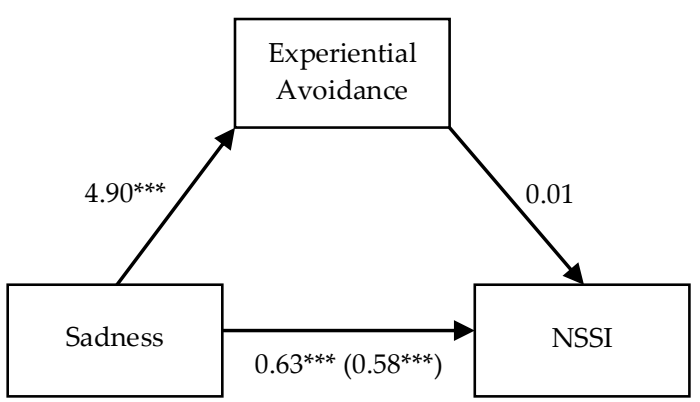

${ }^{* * *} \mathrm{p}<.001$

Figure 24. Unstandardised regression coefficients for the relationship between sadness and NSSI which is not mediated by experiential avoidance. The unstandardised regression coefficient for the relationship between sadness and NSSI controlling for experiential avoidance is in parentheses. Sobel's test $=0.64, \mathrm{SE}=0.06, \mathrm{p}=.52$.

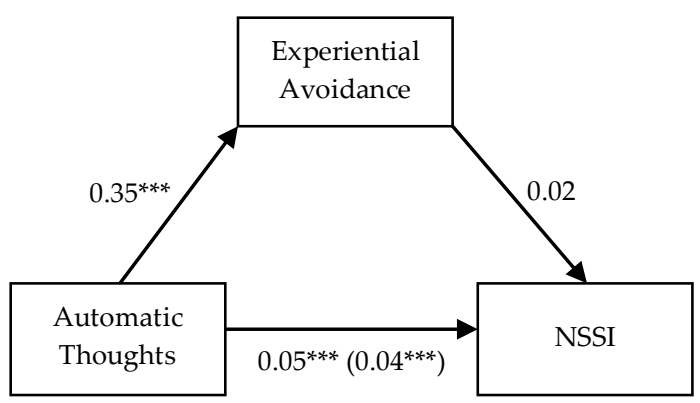

${ }^{* * *} \mathrm{p}<.001$

Figure 26. Unstandardised regression coefficients for the relationship between automatic thoughts and NSSI which is not mediated by experiential avoidance. The unstandardised regression coefficient for the relationship between automatic thoughts and NSSI controlling for experiential avoidance is in parentheses. Sobel's test $=0.88, \mathrm{SE}=0.06, \mathrm{p}=.38$.

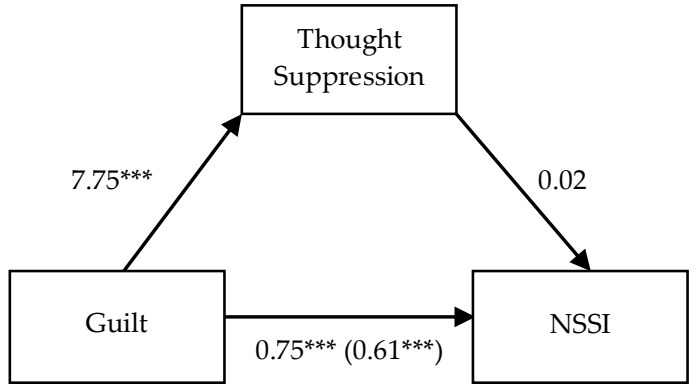

${ }^{* * *} \mathrm{p}<.001$

Figure 23. Unstandardised regression coefficients for the relationship between guilt and NSSI which is not mediated by thought suppression. The unstandardised regression coefficient for the relationship between guilt and NSSI controlling for thought suppression is in parentheses. Sobel's test $=1.80, \mathrm{SE}=0.07, \mathrm{p}=.07$.

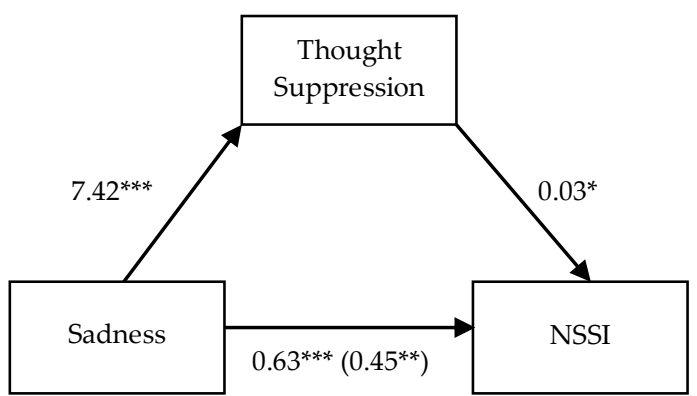

${ }^{*} \mathrm{p}<.05,{ }^{* *} \mathrm{p}<.01,{ }^{* * *} \mathrm{p}<.001$

Figure 25. Unstandardised regression coefficients for the relationship between sadness and NSSI which is partially mediated by thought suppression. The unstandardised regression coefficient for the relationship between sadness and NSSI controlling for thought suppression is in parentheses. Sobel's test $=2.33, \mathrm{SE}=0.07, \mathrm{p}=.02$.

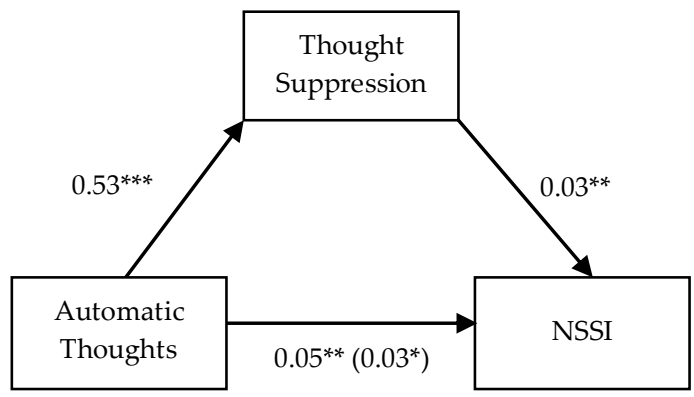

${ }^{*} \mathrm{p}<.05,{ }^{* *} \mathrm{p}<.01,{ }^{* * *} \mathrm{p}<.001$

Figure 27. Unstandardised regression coefficients for the relationship between automatic thoughts and NSSI which is partially mediated by thought suppression. The unstandardised regression coefficient for the relationship between automatic thoughts and NSSI controlling for thought suppression is in parentheses. Sobel's test $=2.91$, SE $=0.06, \mathrm{p}=.004$. 


\section{DISCUSSION}

Non-suicidal self-injury was highly prevalent in this self-selected sample of first year Psychology students, with almost half of the participants reporting a lifetime history of NSSI. These rates are generally higher than those identified in studies of American university students using the same self-injury measure (25\%38\%; Brown, 2009; Gratz et al., 2002), but of course cannot be used to infer true prevalence rates among this population. For instance, a random sample of university students found that only 17\% reported self-injuring in their lifetimes (Whitlock et al., 2006). The participants with a history of NSSI in the current study had typically engaged in more than one type of self-injury; the most common types (i.e., cutting, severe scratching, and sticking sharp objects into one's skin) were generally consistent with what has been reported in other research (Gratz et al., 2002; Hoff \& Muehlenkamp, 2009).

Comparisons between the participants who had, and had not, self-injured revealed several significant differences. To briefly review, it was proposed that people who had self-injured would report experiencing more negative intrapersonal experiences in general than people who had never self-injured and would use more avoidant coping methods. Furthermore, I anticipated that negative emotions and thoughts at T1 would predict engagement in self-injury at T2, and that these relationships may be mediated by dispositional tendencies towards avoidant coping.

\subsection{Experience of negative emotions and thoughts}

The intensity and frequency of negative intrapersonal experiences reported by participants not only differed according to whether or not they had self-injured, but also according to how recently they had self-injured. Participants who had selfinjured in the last 12 months consistently reported the highest levels of general negative affect, emotions (i.e., fear, hostility, guilt, and sadness), and automatic thoughts compared to those who had self-injured more than 12 months ago or never self-injured. 
The same pattern of results was observed for negative emotional experiences that had occurred in the past week. Symptoms of depression, stress, and anxiety were significantly more common among participants who had self-injured within the past 12 months than those who had a lifetime or no history of NSSI. In contrast, participants who had self-injured more than 12 months ago did not differ on symptoms of depression, anxiety, or stress from participants who had never selfinjured. Moreover, participants with a lifetime history of NSSI did not report experiencing more negative automatic thoughts than participants who had never self-injured.

However, these participants did endorse higher negative affect, hostility, guilt, and sadness than those without a history of self-injury, providing evidence that there are some significant differences in the general emotional experiences of people who have self-injured more than a year ago compared to people who have never self-injured. Taken together, these results show that people who have selfinjured report significantly more intense, aversive emotions and a higher frequency of negative automatic thoughts than people who have not self-injured, and that these negative emotional and cognitive experiences are heightened for those with a more recent history of NSSI.

These results are consistent with studies that show NSSI is associated with depression and anxiety disorders (Hintikka et al., 2009; Hoff \& Muehlenkamp, 2009), and with the wealth of evidence that self-injury is primarily used as a strategy to deal with intense, negative affect (Klonsky, 2007). Given that negative cognitions feature prominently in both depression and anxiety (Dozois, Seeds, \& Collins, 2009), it is unsurprising that the people who self-injured most recently also reported experiencing the highest frequency of negative automatic thoughts.

\subsection{Tendencies towards avoidant coping}

As hypothesised, participants who had self-injured were significantly more likely to show a propensity towards avoidant coping strategies than participants who had never self-injured. However, this difference was only apparent with four 
out of the six coping styles examined. Experiential avoidance, thought suppression, substance use, and behavioural disengagement were more commonly endorsed by participants who had self-injured and varied as a function of self-injury recency. This lends further support to the contention outlined within the EAM that self-injury is best understood as one of several avoidant behaviours in a functional response class (Chapman et al., 2006).

In contrast, the use of self-distraction and denial did not differ between the groups. The absence of a significant difference for coping through self-distraction was particularly surprising in light of the evidence that suggests NSSI is an effective form of distraction (Brown et al., 2002; Polk \& Liss, 2009). It may be that selfdistraction is a commonly used coping strategy for people with and without a history of self-injury, but that the difference lies in the behaviours that people engage in to successfully distract themselves. For example, people without a history of NSSI may routinely distract themselves through watching TV or exercising, whereas people who self-injure may routinely distract themselves through cutting or burning.

\subsection{Do negative emotions and thoughts cause self-injury?}

It is clear from the results that participants who had self-injured within the past 12 months were the most likely to endorse experiencing negative emotions and unwanted thoughts, and to report a tendency towards avoidant coping, but the causal direction between these variables cannot be established with cross-sectional data. To allow for causal inferences, two time-points were included in the current study. However, only a few people reported self-injuring between surveys which necessitated revising the data analysis plan.

Although experimental methods are traditionally employed to test causal relationships, these methods are not ethically appropriate for self-injury research. Instead, cross-lagged panel correlations (Kenny, 1975) were used to test causality. Simple cross-lagged panel models of over-time data revealed that both guilt and negative automatic thoughts predicted subsequent self-injury. The self-referential 
characteristics that define guilt and negative automatic thoughts also underpin the self-punishment function of NSSI, which has been consistently identified as one of the most commonly endorsed motivations for self-injury (Briere \& Gil, 1998; Brown et al., 2002; Swannell et al., 2008). Therefore it is possible that guilt and automatic thoughts predict NSSI because these negative intrapersonal experiences are alleviated through self-punishing acts such as self-injury.

\subsection{Specific types of avoidance may underlie self-injury}

Experiential avoidance is conceptualised as a process that involves avoiding emotions, thoughts, or physical sensations (Hayes et al., 1996), and yet it did not act as a mediator in any of the analyses. In contrast, thought suppression, which has been identified as a specific form of experiential avoidance (Chapman et al., 2006; Hayes et al., 1996), partially mediated the relationships between lifetime NSSI and all of the negative intrapersonal experiences assessed, with the exception of guilt. It is intuitive that thought suppression, rather than general experiential avoidance, would mediate the relationship between negative automatic thoughts and NSSI, but it is less intuitive that thought suppression rather than general experiential avoidance would mediate the relationships between negative emotions and NSSI.

It may be that the $\mathrm{AAQ}$ as a measure of experiential avoidance fails to fully capture the defining characteristics of this behavioural process. Indeed, Hayes and colleagues (1996) comment on the limitations associated with reducing generalised avoidant tendencies, which have the potential to manifest in a myriad of specific actions, to a nine item measure. Alternatively, unsuccessful attempts at cognitive regulation through thought suppression (Najmi et al., 2007), rather than general avoidance, may be one of the key mechanisms through which negative emotions lead to self-injurious behaviour. Negative emotions may trigger, or be triggered by, negative cognitions, which in turn could lead to thought suppression. However, given that thought suppression is more likely to increase than decrease unwanted thoughts, self-injury may be subsequently utilised as a more effective avoidant coping strategy (Najmi et al., 2007). 


\subsection{Strengths}

Given the paucity of longitudinal studies on NSSI, conducting this study across two time points was a methodological strength. As predictors of new incidents of NSSI, negative automatic thoughts and guilt likely functioned as EOs or SD's for self-injurious episodes. Future studies should further examine the role of self-referential thoughts and emotions in predicting NSSI. Certainly, the inclusion of the short version of the Automatic Thoughts Questionnaire (Netemeyer et al., 2002) in the survey was a strength of the current study as few researchers have examined the role of cognitions in precipitating NSSI and none, to my knowledge, have investigated the predictive value of negative automatic thoughts. This is clearly an important area for future research.

\subsection{Limitations}

Given that I have discussed the analytic limitations imposed by the small sample of people who reported new episodes of NSSI at T2 several times in this chapter, I will not dwell on these here. Suffice it to say that future research studies designed to test similar hypotheses should take into account the need for larger samples or longer time periods between survey completions. Indeed, two months was an arbitrary time limit, chosen for pragmatic rather than theoretical reasons.

Another pragmatic choice-conducting this study with only university students - is a limitation in that these results may not generalise to other populations. However, this is not necessarily a flaw given the atypically high rates of self-injurious behaviours and psychopathology among university students compared with other community groups (Briere \& Gil, 1998; Stallman, 2010; Whitlock et al., 2006). This suggests that researchers studying NSSI should pay particular attention to university students.

\subsection{Conclusions}

Negative intrapersonal experiences and avoidant coping styles were shown to vary not only as a function of NSSI history, but also as a function of NSSI recency. Particular negative intrapersonal experiences (i.e., automatic thoughts and guilt) also 
predicted new episodes of self-injury. Finally, thought suppression was identified as a partial mediator of relationships between negative intrapersonal experiences and NSSI, although these results need to be replicated using over-time data. These results have a number of clinical implications.

Students with a history of NSSI appear to be contending with significantly higher levels of general negative affect, aversive emotions, and cognitions than students who have never self-injured. Although these levels are not necessarily clinically significant in all cases, these students may be at a heightened risk of future self-injury episodes given their higher baseline rate of negative intrapersonal experiences (Brown et al., 2007). Clinicians should be mindful that even those students who have not self-injured in a year or more may still be struggling to regulate their emotions and cognitions. Other avoidant coping strategies, such as substance use, should also be assessed in students who have self-injured as these may be substituting for NSSI (Chapman et al., 2006). 


\section{CHAPTER SEVEN}

\section{General discussion}

"All of us tend to use the behavior that was effective in the past at getting the outcome we desire." (Cipani \& Schock, 2007, p. 72)

\section{INTRODUCTION}

Does NSSI function primarily as an experientially avoidant behaviour within Aotearoa New Zealand? Based on the three empirical studies I conducted for this thesis, the answer is yes; for many people, on a majority of occasions, self-injury provided an escape from unwanted, negative emotions and thoughts. Of course, as is to be expected, one functional model does not fit all episodes of self-injury. Intrapersonal escape/avoidance did predominate across studies, but some participants' self-injurious behaviours also functioned as an adrenalin rush reminiscent of a drug high (i.e., intrapersonal access), a visceral form of communicating to others when support was needed (i.e., interpersonal access), and an escape from taxing demands or personal responsibilities (i.e., interpersonal escape/avoidance).

Interviewing people for my first study about their experiences of NSSI provided a rich, detailed, and highly nuanced foundation for the subsequent studies in this thesis. Indeed, it was impossible to do justice to the complexity inherent in each person's most recent episode of self-injury, let alone the learning histories that preceded those episodes. Nonetheless, functionally assessing specific episodes of self-injurious behaviour did give me an understanding of why participants were motivated to injure themselves, and allowed me to hypothesise about the ways in which their behaviours were being reinforced and maintained over time.

It was apparent from my analysis that complex and, in many cases, concurrent reinforcement schedules were operating for participants. This reinforcement, for the most part, was intrapersonally mediated; interpersonal functions were atypical. A minority of participants did report accessing attention or 
support subsequent to their self-injury, which may have reinforced their behaviour. However, this support or attention was often temporally delayed and seldom identified as a motivating factor behind their self-injury, making it less likely that these behaviours served an interpersonal function.

Intrapersonal functions once again predominated over interpersonal functions in my second study, a survey of people from across Aotearoa New Zealand, who had all self-injured within the past 12 months. Participants endorsed intrapersonal functions, for both their most recent and global self-injury episodes, significantly more often than interpersonal functions. More specifically, they most often reported self-injuring to regulate affect, punish themselves, and mark their distress; motivations for NSSI that were similarly reported in my interview study. Participants also retrospectively reported decreased negative affect and cognitions following their most recent episode of self-injury, which supports Chapman and colleagues' (2006) proposal that NSSI is primarily maintained through negative reinforcement and thus functions as a form of experiential avoidance.

Consistent with prior conceptualisations of self-injury as a self-help strategy (Favazza \& Conterio, 1988), one of the two themes identified from a thematic analysis of the open-ended, survey responses detailing the consequences of NSSI was self becomes helper. These positive consequences of NSSI appeared to be both intrapersonally and interpersonally mediated. Participants utilised self-injury to regulate emotions, as well as access support and/or treatment. Furthermore, a few participants identified the presence of a physical wound as a positive consequence of self-injury. However, participants also identified a number of aversive consequences of their most recent episode of NSSI, which were captured in the second theme, self becomes transgressor. Self-injury was perceived by many participants as a transgression of socio-cultural norms and following their NSSI episode, people reported needing to conceal evidence of their self-injury from others, being judged by others for self-injuring, and feeling concerned that their behaviour had caused others to suffer. 
The paradox of self-injury as a behaviour that diminishes and provokes distress was apparent in both my interview and survey studies. Although the aversive consequences engendered by self-injury could have functioned to suppress the behaviour, this seemed unlikely in the majority of cases since they typically followed reinforcing consequences. Instead, it is probable that many of the participants would have self-injured again, especially in light of the powerful intrapersonal functions served by their self-injury.

My first two studies provided compelling evidence of the utility of NSSI as a coping strategy for negative emotions and cognitions, leading me to question whether people who self-injure could be differentiated from those who do not selfinjure on the basis of their general intrapersonal experiences and coping styles. For my final study, I compared the emotional, cognitive, and coping experiences of people with a history of NSSI to those who have never self-injured to determine whether there were any significant differences between these two groups. Building on the results of my interview and survey analyses, my third study focused on investigating the broader emotional and cognitive experiences of people who selfinjure, and whether these experiences would predict engagement in NSSI over time.

People with a history of self-injury did report experiencing significantly higher levels of negative emotions and thoughts on a general basis than people who did not have a history of NSSI, and they were also significantly more likely to use avoidant coping strategies, such as substance use and thought suppression. The strength of these differences varied according to NSSI recency. Furthermore, negative automatic thoughts and guilt predicted new episodes of self-injury, lending weight to the key premise of the EAM (Chapman et al., 2006) that people self-injure to decrease or eliminate negative affect, but also emphasising the importance of negative cognitions in precipitating self-injury. Finally, cross-sectional analyses demonstrated that thought suppression acted as a partial mediator of the relationship between negative intrapersonal experiences and NSSI. 
Taken together, the findings from my three studies help to support and further clarify the extant literature on the functions of self-injury (Klonsky, 2009; Klonsky \& Glenn, 2009; Nock \& Prinstein, 2005), as well as provide insight into the cognitive antecedents and consequences of self-injurious behaviours, which have been largely neglected in the NSSI literature. Although this preliminary research into the functions of NSSI in Aotearoa New Zealand is a useful starting point, several questions remain open to debate. In particular, I will discuss what the self-injury mindset may tell us about the interrelationship between emotions and thoughts, where NSSI 'fits' in relation to other coping strategies, whether certain coping strategies (such as NSSI) qualify as disorders, and the clinical implications of conceptualising self-injury as an experientially avoidant behaviour. Finally, the strengths and limitations of my studies will be considered within the context of directions for future research.

\section{THE SELF-INJURY MINDSET}

The difficulty of understanding how the complex interrelationship between emotions and cognitions impacts on the initiation, reinforcement, and maintenance of self-injurious behaviours is an issue which has surfaced repeatedly throughout this thesis. As identified by Nock (2008), few studies examine the cognitive precipitants of self-injury, with many researchers focusing almost exclusively on how self-injury functions as an emotion regulation strategy. However, my research demonstrates that ruling out, or downplaying, the role of cognitions in self-injury is premature. Certainly, the current evidence base about why people self-injure is too limited to conclude, as proposed by Chapman et al. (2006) in the EAM, that selfinjury functions primarily as a form of emotional avoidance.

Instead, it was apparent across my three studies that specific types of cognitions play a significant role in motivating people to engage in self-injury and also allow people to discriminate that reinforcement will be available following selfinjury. Negative automatic thoughts featured prominently in people's narrative descriptions of the antecedents of self-injury and were identified as being 
significantly higher before, than after, self-injury in the cross-sectional, survey study. Furthermore, negative automatic thoughts predicted new episodes of NSSI over time.

These studies suggest that negative automatic thoughts function as EOs for self-injurious behaviours; in other words, they motivate people to injure themselves on purpose. As I discussed in Chapter 4 (see p. 116), EOs have a value-altering effect through determining how potent a specific reinforcer will be in a particular situation and a behaviour-altering effect by increasing the likelihood of a person engaging in behaviours that are typically followed by that reinforcer (Laraway et al., 2003; Michael, 1993; Miltenberger, 2004).

As EOs, negative automatic thoughts therefore not only influence the strength of reinforcement following self-injury, but also how frequently people engage in selfinjurious behaviours. Of course, aversive emotions also appeared to function as EOs in all three of my studies, which highlights the importance of learning more about how emotional and cognitive EOs interact to reinforce NSSI. In the interview study, many people acknowledged that they made a decision to self-injure once they had surpassed a particular threshold of distress. Although they most easily identified the emotional components of this distress, it was clear that negative cognitions were also present.

Part of people's struggle to identify cognitive antecedents seemed to stem from their use of emotion language (e.g., "I felt...") to describe cognitive processes, which is customary for English speakers (Westbrook, Kennerley, \& Kirk, 2007). Two examples from the interview study were provided by Owen and Natalie respectively: "I felt like I was yeah useless" and "I kind of felt like everyone had abandoned me". When couched as feelings, these thoughts appear to be more authoritative and immutable than they actually are which may have exacerbated participants' negative emotional experience prior to self-injuring. This is one way in which negative emotions and thoughts may interact to motivate people to selfinjure. 
Another way in which negative cognitions precipitated self-injury was in their role as $\mathrm{SD}^{\prime} \mathrm{s}$. The evidence for this contention is drawn from the interview study where several participants described how once they had surpassed a particular threshold of distress, their perspective became constricted to the point where they were unable to problem-solve alternative solutions to NSSI. Once in this mindset, they became convinced that self-injuring was the only way they could effectively alleviate their suffering.

The presence of constricted cognition as a precipitant of self-injury supports Chapman et al.'s (2006) hypothesis that people who self-injure may tend towards experiential avoidance because they are unable to generate more helpful coping strategies when emotionally aroused. In these situations, particular thresholds or combinations of emotional and cognitive EOs appeared to activate, or at least occur alongside, verbal rules about the utility of self-injury. Such rules were evidence of the cognitive constriction experienced by some participants prior to self-injury and allowed them to discriminate that relief would be available following NSSI. Chapman et al. (2006) do briefly suggest that the interaction between verbal rules and emotional distress may play a role in maintaining self-injurious behaviour, but they do not address the complexities of this interaction within the EAM, preferring instead to focus on emotional precipitants and the process of emotional avoidance.

However, the interrelationships between emotions and cognitions (both negative, automatic thoughts and verbal rules) evident in my research suggests that it is prudent at this point in time to adopt the more inclusive term of experiential avoidance, which implicates both emotions and thoughts in the reinforcement and maintenance of NSSI, until further research on the role of cognitive processes in selfinjury has been conducted. Given that NSSI does appear to function primarily as a form of experiential avoidance, it may be both clinically and theoretically useful to refer to NSSI as an experientially avoidant coping strategy. 


\section{IS IT USEFUL TO CONCEPTUALISE NSSI AS A COPING STRATEGY?}

Before defining self-injury as a form of avoidant coping, it is first necessary to determine what constitutes a coping strategy and how, if conceptualised as such, NSSI fits with other ways of coping. Unfortunately, the extant coping literature, which is beset with contradictions, provides little clarification about the parameters of different coping mechanisms (see Skinner, Edge, Altman, \& Sherwood, 2003). In an effort to organise this literature to better inform future research on coping, Skinner et al. (2003) comprehensively reviewed the convergences and divergences among 100 measures of coping. Within their conception of coping as "an organizational construct used to encompass the myriad actions individuals use to deal with stressful experiences" (p. 217), Skinner et al. (2003) contend that coping is best viewed as a four-level hierarchy. Adaptive processes are at the top level of the hierarchy; these encompass families of coping, which in turn incorporate ways of coping, which are made up of coping instances. Classifying NSSI within this system (see Figure 28) may prove to be a useful way of organising the different functions of NSSI in relation to other coping behaviours.

At the lowest level, the instances of coping reflect the infinite variations of self-injurious behaviours that people could use to hurt themselves on purpose. These instances of coping are then grouped into ways of coping (e.g., cognitive and emotional avoidance) at the second level of the hierarchy. Other ways of coping identified by Skinner et al. (2003) under the rubric of escape include behavioural avoidance, denial, and wishful thinking. Escape is the third level in the hierarchy and is classified as a family of coping. Families of coping serve particular functions for people, which in turn are linked to adaptive processes (i.e., level four of the hierarchy). Problem-solving, helplessness, and support-seeking are examples of other families of coping, while another adaptive process is gathering and ensuring the availability of social resources (Skinner et al., 2003). For example, the interpersonal access function of NSSI could be classified as gathering social support. 


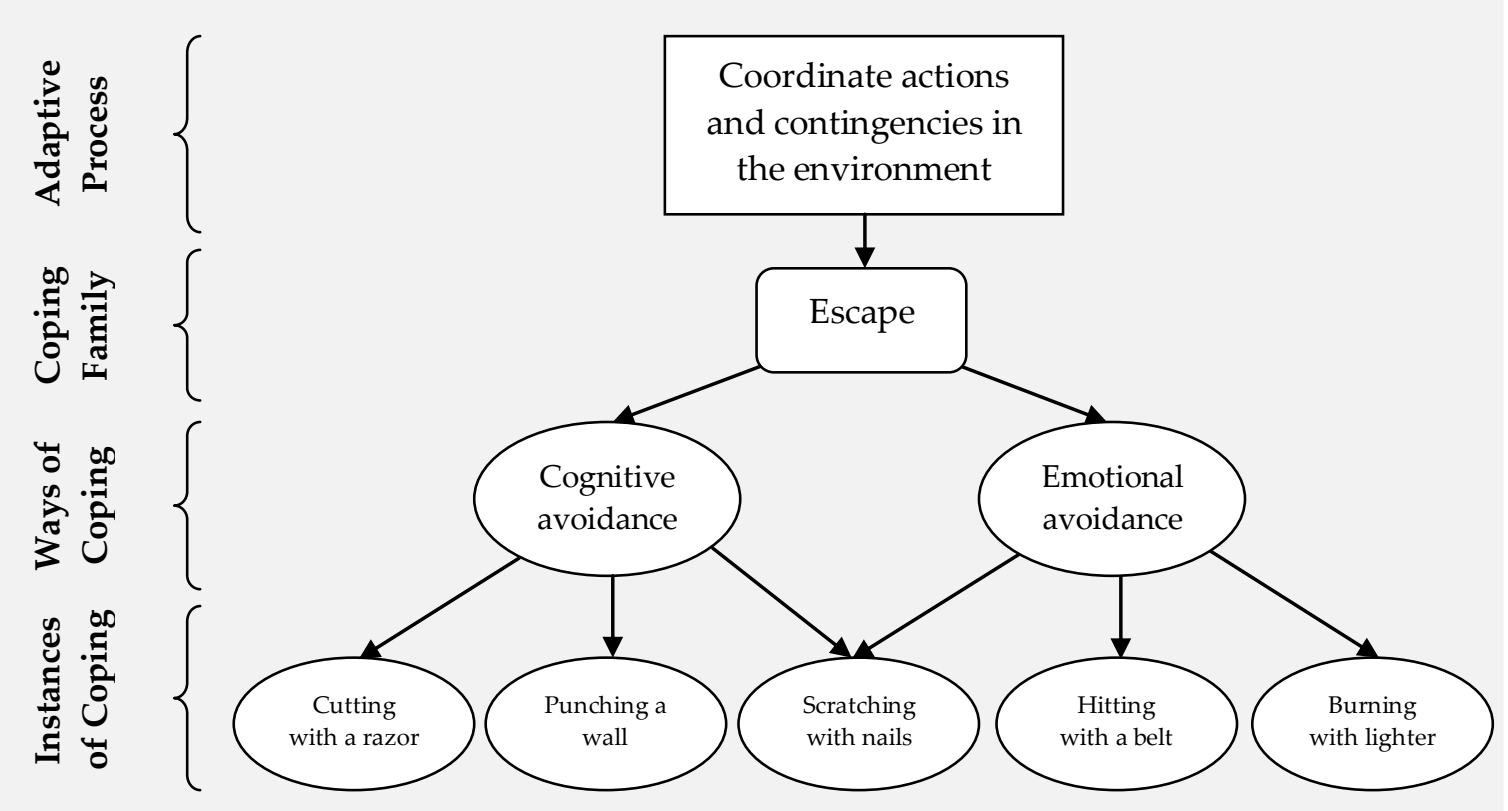

Figure 28. NSSI within an adaptive coping hierarchy. Adapted from "Searching for the structure of coping: A review and critique of category systems for classifying ways of coping," by E.A. Skinner, K. Edge, J. Altman and H. Sherwood, 2003, Psychological Bulletin, 129, p. 218.

Applying Skinner et al.'s (2003) hierarchy to NSSI raises several questions about the ways in which self-injury has been conceptualised to date. For example, Skinner and colleagues categorise emotion regulation within the coping family of self-reliance. They propose that self-reliance is adaptive because it allows people to protect their social resources. Within this taxonomy, it is not clear how NSSI could be described as form of emotion regulation and avoidance at the same time as they are distinct ways of coping. It is possible that instances of NSSI could function to regulate emotions, but those episodes would be separate from instances of NSSI that function as avoidance. However, in the NSSI literature, affect regulation reasons for self-injury are typically subsumed under the function of intrapersonal escape/avoidance (Klonsky \& Glenn, 2009; Nock \& Prinstein, 2004), which implies that self-injury is an emotion regulation strategy because it allows people to successfully escape or avoid their negative emotions. 
Although such a fastidious approach to understanding why people self-injure may seem excessive, it is possible that isolating the mechanisms that underlie NSSI will necessitate clarifying the relationships between specific ways of coping (i.e., cognitive avoidance versus emotional regulation). Undoubtedly, clarification would further develop our understanding of how each of the single-function models (see pp. 68-76) relate to one another. Conceptualising particular self-injury behaviours as instances of coping that serve an adaptive function is also consistent with behavioural models of self-injury.

\section{SHOULD COPING STRATEGIES BE CLASSIFIED AS DISORDERS?}

At the start of this thesis, I discussed the current proposal for an NSSI Disorder to be included in the DSM-5 (American Psychiatric Association, 2010). My understanding of NSSI as a coping behaviour, as stated in Chapter 1 (see p. 26), conflicts with the argument that self-injury should be categorised as a disorder. Certainly, as instances of coping, specific episodes of self-injury do not count as disordered in and of themselves; rather, what could be considered to be maladaptive is the sustained use of self-injury as a form of avoidance. As such, continuously avoiding or escaping distress by self-injuring may qualify as a disorder.

However, if it is the use of avoidance that is deemed unhelpful, then it seems more practical to adopt a functional perspective and view avoidance as a functional diagnostic dimension (cf. Hayes et al., 1996). This is consistent with the argument that NSSI belongs in a functional response class with other avoidant behaviours (Chapman et al., 2006). The alternative structural approach could potentially result in a long list of comorbid disorders, which on the surface may appear to have little in common, but ultimately serve the same function (Dougher \& Hayes, 2000). Furthermore, a diagnosis of NSSI may encourage clinicians to assess and treat selfinjury as a prescribed list of symptoms, rather than as a complex, dynamic way of coping that shifts in response to particular environmental contexts. 


\section{CLINICAL IMPLICATIONS}

The conspicuous lack of empirically supported treatments for NSSI has been commented on by a number of authors (Klonsky \& Muehlenkamp, 2007; Nock, 2010); indeed, the need for research into effective treatments for self-injury has been cited as a reason to classify NSSI as a disorder (Muehlenkamp, 2005). If, as my findings suggest, NSSI does function primarily as an experientially avoidant coping behaviour within Aotearoa New Zealand, it may be advisable for clinicians working here to employ interventions that aim to decrease intrapersonal avoidance.

Two particularly promising interventions for people who utilise self-injury to avoid, or escape from, negative intrapersonal experiences are DBT (Linehan, 1993a, 1993b) and ACT (Hayes et al., 2006). As third-wave cognitive behavioural therapies, DBT and ACT both target experiential avoidance, but the way in which specific skills are conceptualised and taught to clients can differ (Lynch et al., 2006). The philosophy of dialectics lies at the heart of DBT and is concerned with interrelatedness, opposing forces, and the inevitability of change (Linehan, 1993a). Dialectical Behaviour Therapy has been identified as an effective treatment for selfinjury among people diagnosed with BPD (Bohus et al., 2004; Kliem, Kröger, \& Kosfelder, 2010; Stanley, Brodsky, Nelson, \& Dulit, 2007), but no research has been conducted to determine whether it is a similarly efficacious treatment for people, without BPD, who self-injure.

Turning to ACT, a protocol has been developed for treating adolescents who self-injure (Rowland, 2011) but, to my knowledge, the use of ACT to treat NSSI has never been empirically tested. Despite this lack of research, ACT appears to be a highly appropriate treatment for NSSI because it aims to decrease experiential avoidance through promoting psychological flexibility (Hayes et al., 2006). Experiential avoidance, within an ACT framework, is conceptualised as a barrier to acceptance; for someone who routinely escapes negative intrapersonal experiences through self-injury, acceptance would entail remaining in contact with their unwanted feelings or thoughts (Hayes et al., 2006). Psychologically flexible people 
are able to consciously connect with the present moment, and choose to engage in behaviours that enable them to live according their values (Hayes et al., 2006).

Since it is beyond the purpose of this thesis to engage in an in-depth comparison of the potential for these two therapies to reduce self-injurious behaviours, I have instead chosen to discuss how particular strategies, which are routinely used in DBT and ACT protocols, could be used to prevent people from becoming overwhelmed by their negative emotions and thoughts to the point where their cognition constricts and they become trapped in the self-injury mindset. More specifically, I will focus on how teaching people mindfulness, cognitive defusion, and distress tolerance skills may help them to successfully regulate their emotions without resorting to NSSI.

Mindfulness-based approaches to psychopathology, which involve teaching clients to focus their awareness on the present moment, appear to be growing in number and popularity (Chiesa \& Malinowski, 2011). A fundamental element of mindfulness as defined by Kabat-Zinn is the absence of judgement (Elliston, 2002); that is, people are encouraged to develop the ability to simply notice what they are experiencing without judging those experiences as inherently good or bad (Hayes et al., 2006).

Practising mindfulness may reduce the distress associated with particular emotions and thoughts for people who self-injure, which, in turn, could alleviate the sense, as described by participants in my interview study, of mounting pressure. Certainly, if thoughts and emotions are simply noticed, rather than judged as aversive and overwhelming, it is less likely that people will experience the same sense of urgency to escape from these intrapersonal events.

Within a DBT framework, distress tolerance skills evolve out of, and build on, mindfulness training (Linehan, 1993a). Distress tolerance is defined as:

the ability to perceive one's environment without putting demands on it to be different, to experience your current emotional state without attempting to change it, and to observe your own thoughts and action patterns without attempting to stop or control them. (Linehan, 1993a, p. 96) 
It is not difficult to see how distress tolerance may prove to be an essential component of self-injury prevention and intervention efforts in light of my findings that the interaction between intense, negative emotions and self-referential cognitions dominated as NSSI precipitants in studies one and two, and guilt and negative, automatic thoughts actually predicted new episodes of NSSI in study three. Distress tolerance would require people to perceive, observe, and experience their aversive emotions and thoughts instead of engaging in self-injurious behaviours, and is closely related to the notion of acceptance within ACT (Hofman \& Asmundson, 2008).

Cultivating a willingness to remain in contact with distressing thoughts and emotions may not only help people tolerate such experiences, but over time may reduce the likelihood that they reach the threshold of distress where self-injury, in the words of Lucy, becomes inevitable (see p. 130). Certainly, ACT contends that struggling to control unwanted, intrapersonal experiences is futile and counterproductive; such struggles may exacerbate these experiences and, in all likelihood, will heighten distress (Harris, 2006). For instance, as discussed earlier in this thesis (see p. 85), thought suppression usually increases the frequency of unwanted thoughts (Wegner \& Zanakos, 1994).

Cognitive defusion is another skill that may facilitate a reduction in distress over time through decreasing the believability of negative thoughts about oneself, others, and the world (Hayes et al., 2006). When people are fused with their cognitions, these thoughts are interpreted as literal truths or rules, and thus wield immense power over people's behaviour (Harris, 2006). Therefore, the aim of cognitive defusion is to change the relationship that people have with their thoughts, rather than the content or frequency of those thoughts (Hayes et al., 2006).

Teaching cognitive defusion to people who self-injure is likely to be helpful not only in reducing the believability of self-referential thoughts such as, "I am worthless", and thus the distress evoked by such thoughts, but also in challenging the literality and believability of verbal rules such as, "If I want to feel better, then I 
need to hurt myself" (see pp. 131-133). For example, someone could defuse from this verbal rule by labelling it as a thought: "I am having the thought that if I want to feel better, then I need to hurt myself" (Hayes et al., 2006). In sum, ACT and DBT show considerable promise as treatment methods for a wide range of people who selfinjure, but studies in support of this contention are urgently needed.

\section{WHERE TO FROM HERE?}

Research on NSSI can be considered to be in its infancy; sophisticated study designs are needed to further develop our knowledge of why people start selfinjuring and how these behaviours are maintained over time. One of the most critical areas for future research is how to effectively treat people who habitually self-injure, given the dearth of studies on NSSI interventions. In the following sections, I consider how the findings of my studies could be used to inform new research on self-injury and discuss limitations that need to be addressed in the future.

Specifically, improved methods, a continued focus on the EAM and the functions of self-injury, an enhanced understanding of the interrelationship between emotions and cognitions, and more diverse samples are needed in order to develop a robust evidence base of NSSI research.

\subsection{More sophisticated methods are necessary}

In Psychological research, as in literature, unreliable narrators (Booth, 1983) are to be expected. Memories of past events are fallible and the findings of my empirical studies, all of which relied exclusively on self-report methodologies, are undoubtedly influenced to some extent by retrospective bias. Such criticism, however, can be levelled at the majority of research conducted on NSSI to date and is typically an inescapable reality of studies designed to investigate what people think about and how they feel.

Understanding the phenomenology of self-injury is essential to effectively prevent and treat these behaviours and, as such, self-report will always feature within NSSI research. However, the use of increasingly sophisticated methods, such as ecological momentary assessment (see Muehlenkamp et al., 2009; Nock et al., 
2009) will not only help researchers reduce the error associated with retrospective bias, but will also facilitate a deeper understanding of the emotional, cognitive, and situational antecedents and consequences of NSSI. This understanding, in turn, will improve our knowledge of how self-injurious behaviours are reinforced and maintained over time.

One of the limitations of the cross-sectional design of my first two studies is that I was unable to determine whether people's self-injurious behaviours were actually reinforced as I do not know whether they self-injured again. Instead, I could only hypothesise that the majority of the episodes reported by participants were negatively reinforced and, as such, they were likely to engage in further NSSI. Including two time points in my third study was a strength because I was able to test whether specific intrapersonal experiences and coping styles predicted new incidents of self-injury.

Certainly, over-time and longitudinal studies are desperately needed as the majority of work conducted on NSSI to date has been cross-sectional. As a result, we know very little about what factors are causally implicated in the initiation and maintenance of self-injurious behaviours. Preliminary evidence suggests wellestablished NSSI correlates, which have been hypothesised as risk factors for selfinjury, do not actually predict new incidents of self-injury (Glenn \& Klonsky, 2011), making longitudinal studies even more imperative.

\subsection{How do emotions and cognitions interact to precipitate NSSI?}

Although my findings support the utility of conceptualising NSSI primarily as an experientially avoidant behaviour within the context of Aotearoa New Zealand, they also highlight the importance of developing a more sophisticated understanding of how cognitions and emotions interact to precipitate and reinforce self-injurious behaviours. Certainly, the EAM's (Chapman et al., 2006) focus on emotional precipitants appears, in light of my findings about the self-injury mindset, to be overly simplistic. 
Rather, it is clear from my research that the interaction between negative emotions and thoughts led participants to become overwhelmed, and, in some cases, once they surpassed a threshold of emotional intensity, they found it difficult to generate possible coping strategies other then self-injury. An important area of investigation for future research is to examine in greater depth how and why this process of cognitive constriction occurs, as well as the predictive power of verbal rules in this context. More specifically, it would be important to examine withinindividual and between-group differences in how this threshold is reached and then surpassed. For instance, is the tipping point for self-injury completely idiosyncratic, based on individual learning histories and skill deficits, or do gendered, diagnostic, or culture-specific patterns in tipping points exist?

Additionally, it should be noted that the relatively simplistic distinction made between emotions and cognitions when researching NSSI (including my studies) is becoming increasingly outdated when situated within the broader context of the emotion literature (cf. Barrett, 2009; Duncan \& Barrett, 2007). For instance, Duncan and Barrett (2007) argue that at a neurobiological level, affect is a cognitive process and, as such, distinguishing between affect and cognition reflects an epistemological alignment with phenomenology, not ontology. They propose investigating why this distinction holds functional value for people is necessary to better understand the experience of emotions and cognitions. Such investigations within the emotion literature may usefully inform future research on how emotional and cognitive processes interact to precipitate, reinforce, and maintain self-injurious behaviour.

\subsection{Further research on the EAM is warranted}

Despite what is arguably an overly simplistic preoccupation with emotional avoidance, I contend nonetheless that the EAM (Chapman et al., 2006) is a useful framework for both research and clinical practice. However, it is the broader, more inclusive notion of experiential, rather than emotional, avoidance that should be utilised both empirically and clinically. Certainly, specific components of the model, such as why people who self-injure gravitate towards experientially avoidant coping 
strategies in the first place, remain largely untested and would benefit from further research. Understanding why people utilise avoidance over and above other coping strategies provides information for clinicians about what skill deficits could be targeted in treatment.

As I discussed in Chapter 3 (see pp. 82-83), Chapman and colleagues (2006) provide several suggestions as to why people who self-injure may evidence a heightened propensity towards experiential avoidance. It is possible that people who begin self-injuring experience emotions more intensely than people who do not ever self-injure (Chapman et al., 2006). A recent study showed that adolescents who had self-injured were more physiologically reactive when distressed than adolescents who had never self-injured (Nock \& Mendes, 2008). Additionally, I identified in my over-time, survey study that students who had self-injured did report experiencing more intense, negative emotions in general than those who had never self-injured. However, neither of these studies was designed to determine whether these differences in emotional intensity existed prior to the onset of NSSI. Cohort studies with children and young adolescents are needed to examine whether emotional intensity predicts whether people begin engaging in NSSI.

Linked to the notion of emotional intensity is the suggestion that people may be predisposed to NSSI because they are unable to tolerate emotional distress (Chapman et al., 2006). Low distress tolerance has been empirically associated with NSSI (Nock \& Mendes, 2008) and is a target for therapeutic change among people diagnosed with BPD (Linehan, 1993a). Further research needs to be conducted to determine whether low distress tolerance is a risk factor for NSSI.

Finally, it is possible that people who self-injure have a propensity for experientially avoidant coping strategies because they lack access to alternative coping mechanisms or they may possess such skills but find it too difficult to employ them when emotionally overwhelmed (Chapman et al., 2006). The latter hypothesis is more consistent with the findings in my research where people found it difficult to think of alternative coping strategies when they reached specific tipping points. 
Examining these questions within a research context will undoubtedly develop our understanding of why people begin self-injuring and what types of interventions are most appropriate when attempting to reduce self-injurious behaviours.

\subsection{What are the similarities and differences between NSSI and other forms of experiential avoidance?}

Given that NSSI is conceptualised as one of many behaviours within the functional response class of experiential avoidance (Chapman et al., 2006), the similarities and differences between self-injury and functionally equivalent behaviours should be investigated in future research. In particular, understanding why people choose self-injury instead of, or in conjunction with, other experientially avoidant behaviours may have important implications for prevention and treatment efforts. Furthermore, such investigations are necessary to further test the specificity of the EAM (Chapman et al., 2006) to NSSI.

For example, substance use is routinely conceptualised as an experientially avoidant behaviour (see pp. 80, 82, 84-86) and preliminary evidence suggests that people may replace self-injury with substance use (Brown et al., 2007). It is possible that this occurs within the context of developmental shifts; certainly, substance use is a more socially acceptable, avoidant behaviour to engage in as one grows older. However, it is likely that there are other variables, aside from social acceptability, that distinguish self-injury from substance use. Future studies could focus on identifying the factors (i.e., intrapersonal, interpersonal, and situational) that place people at risk of engaging in different forms of experiential avoidance.

\subsection{How do functions of self-injury change over time?}

Applying behavioural theory to the study of NSSI among typically developing populations (Chapman et al., 2006; Klonsky \& Glenn, 2009; Linehan, 1993a, 1993b; Nock \& Prinstein, 2004, 2005) has significantly advanced our understanding of why people self-injure. Longitudinal studies are needed to further develop this evidence base, particularly with regards to how functions of self-injury change within people over time (Nock \& Prinstein, 2004). Given the functions of a 
behaviour are context dependent (Anderson, 2007), it seems probable that the within-person functions of NSSI will be influenced by factors that govern the parameters of people's environments, such as developmental stage.

Ecological momentary assessment techniques will also undoubtedly refine our current understanding of the functions of NSSI as retrospective functional assessments are likely to be subject to memory biases. For example, it is possible that retrospective self-reports may be tapping into the verbal rules people hold about NSSI rather than the behavioural functions. Preliminary results from one of the few ecological momentary assessment studies of NSSI show that the functions of selfinjury are largely consistent with those endorsed in retrospective studies (Nock et al., 2009), but further research is needed to replicate these results.

\subsection{What does NSSI communicate to others?}

Although NSSI does appear to function primarily as an experientially avoidant behaviour both within Aotearoa New Zealand and in international samples (Klonsky, 2007, 2011; Nock \& Prinstein, 2004), interpersonal functions can play an important role in the initiation and maintenance of self-injury. In both my interview and cross-sectional survey studies, there were a number of participants who described utilising self-injury as a means of avoiding interpersonal responsibilities or seeking support.

To better understand the complexity of how interpersonal functions of NSSI are reinforced, maintained, or punished, it would be useful to conduct further research on how and why NSSI functions as a form of communication to others. Additionally, it would be valuable to compare the semiotics of self-injury (i.e., what the behaviours signify to others) across cultures, sub-cultures, genders, and age groups, and how often messages conveyed through NSSI are received as intended.

Certainly, several participants in my interview study provided examples of how the reasons behind their self-injurious behaviour had been misinterpreted by 
others. Angela described being labelled as $\mathrm{emo}^{42}$ by people who saw evidence of her self-injury, a judgement she found "very offensive". She described self-injury, when associated with this subculture, as a public, group activity that was discussed "almost boastfully". For Angela, emo self-injury appeared to be distasteful because it primarily fulfilled an attention-seeking function. This directly contrasted with her use of NSSI as a self-help strategy.

The belief that some groups use NSSI as a status symbol was echoed in Emily's observation that the popularity of self-injury at her all-girls' secondary school was evidence of a "glamorisation of dysfunction". While self-injury contagion has been studied among clinical populations (Matthews, 1968; Rosen \& Walsh, 1989), the normalisation of self-injury within particular peer groups in community-based samples remains largely unexamined. Certainly, if such normalisation is prevalent, identifying what factors place young people at risk of succumbing to peer pressure to self-injure would be an important area for future research.

\subsection{Diverse samples are needed}

As commented on earlier in this thesis, much of the research on NSSI has been conducted with female Caucasians (Shaw, 2002); my studies are no exception. The majority of the participants in all three of the studies I conducted were female and the samples lacked ethnic diversity. Furthermore, in my third study, participants who did not complete the Time 2 survey were significantly more likely to be male, perhaps suggesting that males are less willing to participate in research on NSSI. Unwillingness to participate could stem from perceptions that self-injury is a predominantly female problem or a reluctance to disclose self-injury.

Although Pākehā are in the majority in Aotearoa New Zealand, they are overrepresented in my research compared to general population statistics (Bascand, 2007). As such, the lack of ethnic diversity observed across my three studies does not

\footnotetext{
${ }^{42}$ The word emo signifies a disparaging stereotype of someone who is overly sensitive or emotional (Emo, n.d.).
} 
reflect the current social demographics of Aotearoa New Zealand. This limitation should be addressed in future research especially given that definitions of NSSI are socio-culturally bound, as discussed in Chapter 1. Within Aotearoa New Zealand, researchers have an obligation to conduct themselves in accordance with the principles of the Treaty of Waitangi and particular attention thus needs to be paid to understanding Māori conceptualisations of NSSI and how these behaviours may manifest among Māori. Certainly, researching and treating self-injury as an experientially avoidant behaviour may not be appropriate for Māori or other ethnic groups, particularly if avoidant behaviours are considered socially acceptable or culturally adaptive in such communities.

\section{CONCLUSION}

Non-suicidal self-injury is an important topic for future research as it appears to be a highly prevalent behaviour among certain groups and our understanding of why people begin, and continue, to self-injure is limited. However, I believe that it is equally important not to sensationalise the prevalence of NSSI in our communities; although many researchers unproblematically assert that the incidence of NSSI is rising, there is little evidence to confirm this suspicion. Alarmist statements such as that contained in one journal article title which referred to adolescent NSSI as "the latest epidemic [emphasis added] to assess and treat" (Miller \& Smith, 2008, p. 178) misrepresent the current state of knowledge in this area.

Certainly, I am cognisant that the explosion of research interest in NSSI may have helped to establish self-injury as one of the behaviours in the symptom pool of Western cultures, thus legitimising it as a signifier of distress (Shorter, 1987) and possibly fuelling an increase in the prevalence of these behaviours. Researchers have a responsibility to reflect on how they present NSSI in scientific discourse as these forms of knowledge have the potential to exert a significant influence over the way in which people behave (Watters, 2010). In spite of these concerns, further research into NSSI is warranted to try to prevent people from choosing it as a coping strategy 
and to ensure that people who do engage in repeated self-injury have access to effective, evidence-based treatments. 


\section{References}

Adrian, M., Zeman, J., Erdley, C., Lisa, L., \& Sim, L. (2011). Emotional dysregulation and interpersonal difficulties as risk factors for nonsuicidal self-injury in adolescent girls. Journal of Abnormal Child Psychology, 39, 389-400. doi:10.1007/ s10802-010-9465-3

Alexander, N., \& Clare, L. (2004). You still feel different: The experience and meaning of women's self-injury in the context of a lesbian or bisexual identity. Journal of Community E Applied Social Psychology, 14, 70-84. doi:10.1002/ casp.764

American Psychiatric Association. (2000). Diagnostic and statistical manual of mental disorders ( $4^{\text {th }}$ ed., text rev.). Washington, DC: Author.

American Psychiatric Association. (2010). Other disorders. Retrieved from http://www.dsm5.org/ProposedRevision/Pages/OtherDisorders.aspx

Anderson, C.M. (2007).Functional assessment with clinical populations: Current status and future directions. In P. Sturmey (Ed.), Functional analysis in clinical treatment (pp. 455-471). Burlington, MA: Academic Press.

Anderson, N.L. (2009). A test of two models of non-suicidal self-injury (Unpublished master's thesis). Kent State University, Ohio.

Andover, M.S., \& Gibb, B.E. (2010). Non-suicidal self-injury, attempted suicide, and suicidal intent among psychiatric inpatients. Psychiatry Research, 178, 101-105. doi:10.1016/j.psychres.2010.03.019

Andover, M.S., Pepper, C.M., \& Gibb, B.E. (2007). Self-mutilation and coping strategies in a college sample. Suicide and Life-Threatening Behavior, 37, 238-243. doi:10.1521/suli.2007.37.2.238

Andover, M.S., Pepper, C.M., Ryabchenko, K.A., Orrico, E.G., \& Gibb, B.E. (2005). Self-mutilation and symptoms of depression, anxiety, and borderline personality disorder. Suicide and Life-Threatening Behavior, 35, 581-591. 
Andover, M.S., Primack, J.M., Gibb, B.E., \& Pepper, C.M. (2010). An examination of non-suicidal self-injury in men: Do men differ from women in basic NSSI characteristics? Archives of Suicide Research, 14, 79-88. doi:10.1080/1381111090 3479086

Angen, M.J. (2000). Evaluating interpretive inquiry: Reviewing the validity debate and opening the dialogue. Qualitative Health Research, 10, 378-395. doi:10.1177/ 104973230001000308

Antony, M.M., Bieling, P.J., Cox, B.J., Enns, M.W., \& Swinson, R.P. (1998). Psychometric properties of the 42-item and 21-item versions of the Depression Anxiety Stress Scales in clinical groups and a community sample. Psychological Assessment, 10, 176-181. doi:10.1037/1040-3590.10.2.176

Armey, M.F., \& Crowther, J.H. (2008). A comparison of linear versus non-linear models of aversive self-awareness, dissociation, and non-suicidal self-injury among young adults. Journal of Consulting and Clinical Psychology, 76, 9-14. doi:10.1037/0022-006X.76.1.9

Babiker, G., \& Arnold, L. (1997). The language of injury: Comprehending self-mutilation. Leicester, UK: BPS Blackwell.

Baetens, I., Claes, L., Willem, L., Muehlenkamp, J., \& Bijttebier, P. (2011). The relationship between non-suicidal self-injury and temperament in male and female adolescents based on child- and parent-report. Personality and Individual Differences, 50, 527-530. doi:10.1016/j.paid.2010.11.015

Bandura, A. (1977). Social learning theory. Oxford, England: Prentice-Hall.

Baron, R.M., \& Kenny, D.A. (1986). The moderator-mediator variable distinction in social psychological research: Conceptual, strategic, and statistical considerations. Journal of Personality and Social Psychology, 51, 1173-1182. doi:10.1037/0022-3514.51.6.1173

Barrett, L.F. (1998). Discrete emotions or dimensions? The role of valence focus and arousal focus. Cognition and Emotion, 12, 579-599. 
Barrett, L.F. (2009). Variety is the spice of life: A psychological construction approach to understanding variability in emotion. Cognition and Emotion, 23, 1284-1306. doi:10.1080/02699930902985894

Barrett, L.F., Gendron, M., \& Huang, Y. (2009). Do discrete emotions exist? Philosophical Psychology, 22, 427-437. doi:10.1080/09515080903153634

Bascand, G. (2007). Profile of New Zealander responses, ethnicity question: 2006 census. Wellington: Statistics New Zealand. Retrieved from http://www.stats.govt.nz/browse_for_stats/population/census_counts/reviewmeasurement-of-ethnicity/papers.aspx

Beautrais, A.L., \& Fergusson, D.M. (2006). Indigenous suicide in New Zealand. Archives of Suicide Research, 10, 159-168. doi:10.1080/13811110600556913

Bebbington, P.E., Minot, S., Cooper, C., Dennis, M., Meltzer, H., Jenkins, R., \& Brugha, T. (2010). Suicidal ideation, self-harm and attempted suicide: Results from the British psychiatric morbidity survey 2000. European Psychiatry, 25, 427-431. doi:10.1016/j.eurpsy.2009.12.004

Ben-Zeev, D., Young, M.A., \& Corrigan, P.W. (2010). DSM-V and the stigma of mental illness. Journal of Mental Health, 19, 318-327. doi:10.3109/09638237. 2010.492484

Blumberg, S.J. (2000). The white bear suppression inventory: Revisiting its factor structure. Personality and Individual Differences, 29, 943-950. doi:10.1016/S01918869(99)00245-7

Bohus, M., Haaf, B., Simms, T., Limberger, M.F., Schmahl, C., Unckel, C.,...\& Linehan, M.M. (2004). Effectiveness of inpatient dialectical behavioral therapy for borderline personality disorder: A controlled trial. Behaviour Research and Therapy, 42, 487-499. doi:10.1016/S0005-7967(03)00174-8

Booth, W.C. (1983). The rhetoric of fiction. Chicago: University of Chicago Press.

Boxer, P. (2010). Variations in risk and treatment factors among adolescents engaging in different types of deliberate self-harm in an inpatient sample. 
Journal of Clinical Child and Adolescent Psychology, 39, 470-480.

doi:10.1080/15374416.2010.486302

Braun, V., \& Clarke, V. (2006). Using thematic analysis in psychology. Qualitative Research in Psychology, 3, 77-101.

Brausch, A.M., \& Gutierrez, P.M. (2010). Differences in non-suicidal self-injury and suicide attempts in adolescents. Journal of Youth and Adolescence, 39, 233-242. doi:10.1007/s10964-009-9482-0

Briere, J., \& Gil, E. (1998). Self-mutilation in clinical and general population samples: Prevalence, correlates, and functions. American Journal of Orthopsychiatry, 68, 609-620.

British Psychological Society. (2007). Report of the working party on conducting research on the Internet: Guidelines for ethical practice in psychological research online. Retrieved from http://www.bps.org.uk/sites/default/files/documents/ conducting_research_on_the_internet-guidelines_for_ethical_practice_in_ psychological_research_online.pdf.

Brown, S.A. (2009). Personality and non-suicidal deliberate self-harm: Trait differences among a non-clinical population. Psychiatry Research, 169, 28-32. doi:10.1016/j.psychres.2008.06.005

Brown, M.Z., Comtois, K.A., \& Linehan, M.M. (2002). Reasons for suicide attempts and nonsuicidal self-injury in women with borderline personality disorder. Journal of Abnormal Psychology, 111, 198-202. doi:10.1037//0021-843X.111.1.198

Brown, G.K., Henriques, G.R., Sosdjan, D., \& Beck, A.T. (2004). Suicide intent and accurate expectations of lethality: Predictors of medical lethality of suicide attempts. Journal of Consulting and Clinical Psychology, 72, 1170-1174. doi:10.1037/0022-006X.72.6.1170

Brown, M.Z., Linehan, M.M., Comtois, K.A., Murray, A., \& Chapman, A.L. (2009). Shame as a prospective predictor of self-inflicted injury in borderline personality disorder: A multi-modal analysis. Behaviour Research and Therapy, 47, 815-822. doi:10.1016/j.brat.2009.06.008 
Brown, S.A., Williams, K., \& Collins, A. (2007). Past and recent deliberate self-harm: Emotion and coping strategy differences. Journal of Clinical Psychology, 63, 791803. doi:10.1002/jclp.20380

Carver, C.S. (1997). You want to measure coping but your protocol's too long:

Consider the Brief COPE. International Journal of Behavioral Medicine, 4, 92-100. doi: 10.1207/s15327558ijbm0401_6

Carver, C.S., Scheier, M.F., \& Weintraub, J.K. (1989). Assessing coping strategies: A theoretically based approach. Journal of Personality and Social Psychology, 56, 267-283. doi:10.1037/0022-3514.56.2.267

Chapman, A.L., \& Dixon-Gordon, K.L. (2007). Emotional antecedents and consequences of deliberate self-harm and suicide attempts. Suicide and LifeThreatening Behavior, 37, 543-552.

Chapman, A.L., Gratz, K.L., \& Brown, M.Z. (2006). Solving the puzzle of deliberate self-harm: The experiential avoidance model. Behaviour Research and Therapy, 44, 371-394. doi:10.1016/j.brat.2005.03.005

Chapman, A.L., Specht, M.W., \& Cellucci, T. (2005). Borderline personality disorder and deliberate self-harm: Does experiential avoidance play a role? Suicide and Life-Threatening Behavior, 35, 388-399. doi:10.1521/suli.2005.35.4.388

Chew-Graham, C., Bashir, C., Chantler, K., Burman, E., \& Batsleer, J. (2002). South Asian women, psychological distress and self-harm: Lessons for primary care trusts. Health $\mathcal{E}$ Social Care in the Community, 10, 339-347.

Chiesa, A., \& Malinowski, P. (2011). Mindfulness-based approaches: Are they all the same? Journal of Clinical Psychology, 67, 404-424. doi: 10.1002/jclp.20776

Cipani, E., \& Schock, K.M. (2007). Functional behavioral assessment, diagnosis, and treatment: A complete system for education and mental health settings. New York: Springer Publishing Company.

Claes, L., Houben, A., Vandereycken, W., Bijttebier, P., \& Muehlenkamp, J. (2010). Brief report: The association between non-suicidal self-injury, self-concept 
and acquaintance with self-injurious peers in a sample of adolescents. Journal of Adolescence, 33, 775-778. doi:10.1016/j.adolescence.2009.10.012

Claes, L., Klonsky, E.D., Muehlenkamp, J., Kuppens, P., \& Vandereycken, W. (2010). The affect-regulation function of nonsuicidal self-injury in eating-disordered patients: Which affect states are regulated? Comprehensive Psychiatry, 51, 386392. doi:10.1016/j.comppsych.2009.09.001

Claes, L., \& Vandereycken, W. (2007). Self-injurious behavior: Differential diagnosis and functional differentiation. Comprehensive Psychiatry, 48, 137-144. doi:10.1016/j.comppsych.2006.10.009

Claes, L., Vandereycken, W., \& Vertommen, H. (2007). Self-injury in female versus male psychiatric patients: A comparison of characteristics, psychopathology and aggression regulation. Personality and Individual Differences, 42, 611-621. doi:10.1016/j.paid.2006.07.021

Clatworthy, J., Buick, D., Hankins, M., Weinman, J., \& Horne, R. (2005). The use and reporting of cluster analysis in health psychology: A review. British Journal of Health Psychology, 10, 329-358.

Clinchy, B.M. (2002). Revisiting women's ways of knowing. In B.K. Hofer, \& P.R. Pintrich (Eds.). Personal epistemology: The psychology of beliefs about knowledge and knowing (pp. 63-87). Mahwah, NJ: Lawrence Erlbaum Associates.

Cone, J.D. (1997). Issues in functional analysis in behavioral assessment. Behaviour Research and Therapy, 35, 259-275. doi:10.1016/S0005-7967(96)00101-5

Cresswell, M. (2005). Psychiatric "survivors" and testimonies of self-harm. Social Science E Medicine, 61, 1668-1677. doi:10.1016/j.socscimed.2005.03.033

Crowell, S.E., Beauchaine, T.P., \& Linehan, M.M. (2009). A biosocial developmental model of borderline personality: Elaborating and extending Linehan's theory. Psychological Bulletin, 135, 495-510. doi:10.1037/a0015616.A

Croyle, K.L., \& Waltz, J. (2007). Subclinical self-harm: Range of behaviors, extent, and associated characteristics. American Journal of Orthopsychiatry, 77, 332-342. doi:10.1037/0002-9432.77.2.332 
Cuper, P., Merwin, R., \& Lynch, T. (2007). Personality disorders. In P. Sturmey (Ed.), Functional analysis in clinical treatment (pp. 403-427). Burlington, MA: Academic Press.

Curtis, C. (2003). Non-fatal suicidal behaviour: Initiation, cessation and prevention (Unpublished PhD thesis). University of Waikato, New Zealand.

Deliberto, T.L., \& Nock, M.K. (2008). An exploratory study of correlates, onset, and offset of non-suicidal self-injury. Archives of Suicide Research, 12, 219-231. doi:10.1080/13811110802101096

Dougher, M.J., \& Hackbert, L. (2000). Establishing operations, cognition, and emotion. The Behavior Analyst, 23, 11-24.

Dougher, M.J., \& Hayes, S.C. (2000). Clinical behavior analysis. In M.J. Dougher (Ed.), Clinical Behavior Analysis (pp. 11-25). Reno, NV: Context Press.

Dougherty, D.M., Mathias, C.W., Marsh-Richard, D.M., Prevette, K.N., Dawes, M.A., Hatzis, E.S.,...Nouvion, S.O. (2009). Impulsivity and clinical symptoms among adolescents with non-suicidal self-injury with or without attempted suicide. Psychiatry Research, 169, 22-27. doi:10.1016/j.psychres.2008.06.011

Dozois, D.J.A., Seeds, P.M., \& Collins, K.A. (2009). Transdiagnostic approaches to the prevention of depression and anxiety. Journal of Cognitive Psychotherapy, 23, 44-59. doi:10.1891/0889-8391.23.1.44

Duncan, S., \& Barrett, L.F. (2007). Affect is a form of cognition: A neurobiological analysis. Cognition E Emotion, 21, 1184-1211. doi:10.1080/02699930701437931

Elliston, P. (2002). A conversation with Jon Kabat-Zinn, Saki Santorelli and Mark Williams. Spirituality and Health International, 3, 18-29. doi: 10.1002/shi.80

Emo. (n.d.). Retrieved March 11, 2012, from Wiktionary: http://en.wiktionary.org/ wiki/emo

Farmer, R.F., \& Latner, J.D. (2007). Eating disorders. In P. Sturmey (Ed.), Functional analysis in clinical treatment (pp. 379-402). Burlington, MA: Academic Press. 
Farrelly, T., \& Francis, K. (2009). Definitions of suicide and self-harm behavior in an Australian Aboriginal community. Suicide and Life-Threatening Behavior, 39, 182-189. doi:10.1521/suli.2009.39.2.182

Favazza, A.R. (1989). General and theoretical issues: Normal and deviant selfmutilation. Transcultural Psychiatry, 26, 113-127.

Favazza, A. (1987). Bodies under siege: Self-mutilation in culture and psychiatry. Baltimore: Johns Hopkins University Press.

Favazza, A. (1996). Bodies under siege: Self-mutilation and body modification in culture and psychiatry (2 ${ }^{\text {nd }}$ ed.). Baltimore: Johns Hopkins University Press.

Favazza, A. (2011). Bodies under siege: Self-mutilation, nonsuicidal self-injury, and body modification in culture and psychiatry ( $3^{\text {rd }} \mathrm{ed}$.). Baltimore: Johns Hopkins University Press.

Favazza, A.R., \& Conterio, K. (1988). The plight of chronic self-mutilators. Community Mental Health Journal, 24, 22-30.

Favazza, A.R., \& Conterio, K. (1989). Female habitual self-mutilators. Acta Psychiatrica Scandinavica, 79, 283-289.

Favazza, A.R., \& Rosenthal, R.J. (1993). Diagnostic issues in self-mutilation. Hospital and Community Psychiatry, 44, 134-140.

Festinger, L.A. (1978). Theory of cognition. Evanston, IL: Row, Peterson.

Field, A. (2009). Discovering statistics using SPSS (3 $3^{\text {rd }}$ ed.). London: SAGE.

Firestone, R.W., \& Seiden, R.H. (1990). Suicide and the continuum of self-destructive behavior. Journal of American College Health, 38, 207-213.

Fliege, H., Kocalevent, R., Walter, O.B., Beck, S., Gratz, K.L., Gutierrez, P.M., \& Klapp, B.F. (2006). Three assessment tools for deliberate self-harm and suicide behavior: Evaluation and psychopathological correlates. Journal of Psychosomatic Research, 61, 113-121. doi:10.1016/j.jpsychores.2005.10.006

Fliege, H., Lee, J., Grimm, A., \& Klapp, B.F. (2009). Risk factors and correlates of deliberate self-harm behavior: A systematic review. Journal of Psychosomatic Research, 66, 477-493. doi:10.1016/j.jpsychores.2008.10.013 
Follette, W.C., Naugle, A.E., \& Linnerooth, P.J.N. (2000). Functional alternatives to traditional assessment and diagnosis. In M.J. Dougher (Ed.), Clinical Behavior Analysis (pp. 99-125). Reno, NV: Context Press.

Fortune, S.A. (2006). An examination of cutting and other methods of DSH among children and adolescents presenting to an outpatient psychiatric clinic in New Zealand. Clinical Child Psychology and Psychiatry, 11, 407-416. doi:10.1177/ 1359104506064984

Fortune, S., Watson, P., Robinson, E., Fleming, T., Merry, S., \& Denny, S. (2010). Youth'07: The health and wellbeing of secondary school students in New Zealand: Suicide behaviours and mental health in 2001 and 2007. Auckland: The University of Auckland. Retrieved from http://www.youth2000.ac.nz/publications/ reports-1142.htm

Freedenthal, S. (2007). Challenges in assessing intent to die: Can suicide attempters be trusted? OMEGA, 55, 57-70.

Garisch, J. (2010). Youth deliberate self-harm: Interpersonal and intrapersonal vulnerability factors, and constructions and attitudes within the social environment (Unpublished PhD thesis). Victoria University of Wellington, New Zealand.

Garisch, J.A., \& Wilson, M.S. (2010). Vulnerabilities to deliberate self-harm among adolescents: The role of alexithymia and victimization. British Journal of Clinical Psychology, 49, 151-162. doi:10.1348/014466509X441709

Glassman, L.H., Weierich, M.R., Hooley, J.M., Deliberto, T.L., \& Nock, M.K. (2007). Child maltreatment, non-suicidal self-injury, and the mediating role of selfcriticism. Behaviour Research and Therapy, 45, 2483-2490. doi:10.1016/j.brat.2007. 04.002

Glenn, C.R., \& Klonsky, E.D. (2010a). A multimethod analysis of impulsivity in nonsuicidal self-injury. Personality Disorders: Theory, Research, and Treatment, 1, 67-75. doi:10.1037/a0017427

Glenn, C.R., \& Klonsky, E.D. (2010b). The role of seeing blood in non-suicidal selfinjury. Journal of Clinical Psychology, 66, 466-473. doi:10.1002/jclp.20661 
Glenn, C.R., \& Klonsky, E.D. (2011). Prospective prediction of nonsuicidal selfinjury: A 1-year longitudinal study in young adults. Behavior Therapy, 42, 751762. doi:10.1016/j.beth.2011.04.005

Goldfried, M., \& Sprafkin, J. (1974). Behavioral personality assessment. In J.T. Spence, R.C. Carson, \& J.W. Thibaut (Eds.), Behavioral approaches to therapy (pp. 295-321). Morristown, NJ: General Learning Press.

Graff, H., \& Mallin, R. (1967). The syndrome of the wrist cutter. American Journal of Psychiatry, 124, 36-42.

Gratz, K.L. (2001). Measurement of deliberate self-harm: Preliminary data on the deliberate self-harm inventory. Journal of Psychopathology and Behavioral Assessment, 23, 253-263. doi:10.1023/A:1012779403943

Gratz, K.L. (2003). Risk factors for and functions of deliberate self-harm: An empirical and conceptual review. Clinical Psychology: Science and Practice, 10, 192-205. doi:10.1093/clipsy/bpg022

Gratz, K.L. (2006). Risk Factors for deliberate self-harm among female college students: The role and interaction of childhood maltreatment, emotional inexpressivity, and affect intensity/reactivity. American Journal of Orthopsychiatry, 76, 238-250. doi:10.1037/0002-9432.76.2.238

Gratz, K.L., Conrad, S.D., \& Roemer, L. (2002). Risk factors for deliberate self-harm among college students. American Journal of Orthopsychiatry, 72, 128-140. doi:10.1037//0002-9432.72.1.128

Gratz, K.L., Hepworth, C., Tull, M.T., Paulson, A., Clarke, S., Remington, B., \& Lejuez, C.W. (2011). An experimental investigation of emotional willingness and physical pain tolerance in deliberate self-harm: The moderating role of interpersonal distress. Comprehensive Psychiatry, 52, 63-74. doi:10.1016/j. com ppsych.2010.04.009

Grayson, D. (2004). Some myths and legends in quantitative psychology. Understanding Statistics, 3, 101-134. 
Haines, J., Williams, C.L., Brain, K.L., \& Wilson, G.V. (1995). The psychophysiology of self-mutilation. Journal of Abnormal Psychology, 104, 471-489. doi:10.1037/ 0021-843X.104.3.471

Hankin, B.L., \& Abela, J.R.Z. (2011). Nonsuicidal self-injury in adolescence: Prospective rates and risk factors in a 21/2 year longitudinal study. Psychiatry Research, 186, 65-70. doi:10.1016/j.psychres.2010.07.056

Hanley, G.P., Iwata, B.A., \& McCord, B.E. (2003). Functional analysis of problem behavior: A review. Journal of Applied Behavior Analysis, 36, 147-185.

Hanly, A., Pietrusza, C., Gluck, M., \& Whitlock, J. (2011, June). The ethics of self-report surveys assessing non-suicidal self-injury and other sensitive topics. Poster session presented at the 6th annual meeting of the International Society for the Study of Self-Injury (ISSS), New York, NY.

Harris, J. (2000). Self-harm: Cutting the bad out of me. Qualitative Health Research, 10, 164-173. doi:10.1177/104973200129118345

Harris, R. (2006). Embracing your demons: An overview of acceptance and commitment therapy. Psychotherapy in Australia, 12, 2-8.

Hasking, P., Momeni, R., Swannell, S., \& Chia, S. (2008). The nature and extent of non-suicidal self-injury in a non-clinical sample of young adults. Archives of Suicide Research, 12, 208-218. doi:10.1080/13811110802100957

Hatcher, S., Sharon, C., \& Collins, N. (2009). Epidemiology of intentional self-harm presenting to four district health boards in New Zealand over 12 months, and comparison with official data. Australian and New Zealand Journal of Psychiatry, 43, 659-665. doi:10.1080/00048670902970833

Hawton, K., \& Harriss, L. (2008). The changing gender ratio in occurrence of deliberate self-harm across the lifecycle. Crisis, 29, 4-10. doi:10.1027/02275910.29.1.4

Hawton, K., \& Rodham, K. (2006). By their own young hand: Deliberate self-harm and suicidal ideas in adolescents. London: Jessica Kingsley Publishers. 
Hayes, S.C., \& Bissett, R.T. (2000). Behavioral psychotherapy and the rise of clinical behavior analysis. In J. Austin \& J. E. Carr (Eds.), Handbook of applied behavior analysis (pp. 231-245). Reno, NV: Context Press.

Hayes, S.C., Luoma, J.B., Bond, F.W., Masuda, A., \& Lillis, J. (2006). Acceptance and commitment therapy: Model, processes and outcomes. Behaviour Research and Therapy, 44, 1-25. doi:10.1016/j.brat.2005.06.006

Hayes, S.C., Strosahl, K., Wilson, K.G., Bissett, R.T., Pistorello, J., Toarmino, D., Polusny, M.A.,...McCurry, S.M. (2004). Measuring experiential avoidance: A preliminary test of a working model. The Psychological Record, 54, 553-578.

Hayes, S.C., Wilson, K.G., Gifford, E.V., Follette, V.M., \& Strosahl, K. (1996).

Experiential avoidance and behavioral disorders: A functional dimensional approach to diagnosis and treatment. Journal of Consulting and Clinical Psychology, 64, 1152-1168. doi:10.1037/0022-006X.64.6.1152

Heatherton, T.F., \& Baumeister, R.F. (1991). Binge eating as escape from selfawareness. Psychological Bulletin, 110, 86-108. doi:10.1037/0033-2909.110.1.86

Heilbron, N., \& Prinstein, M.J. (2010). Adolescent peer victimization, peer status, suicidal ideation, and nonsuicidal self-injury: Examining concurrent and longitudinal associations. Merrill-Palmer Quarterly, 56, 388-419. doi:10.1353/mpq.0.0049

Henry, J.D., \& Crawford, J.R. (2005). The short-form version of the Depression Anxiety Stress Scales (DASS-21): Construct validity and normative data in a large non-clinical sample. British Journal of Clinical Psychology, 44, 227-239. doi:10.1348/014466505X29657

Herpetz, S. (1995). Self-injurious behavior: Psychopathologial and nosological characteristics in subtypes of self-injurers. Acta Psychiatrica Scandinavica, 91, 57-68. doi:10.1111/j.1600-0447.1995.tb09743.x

Hicks, K.M., \& Hinck, S.M. (2008). Concept analysis of self-mutilation. Journal of Advanced Nursing, 64, 408-413. doi:10.1111/j.1365-2648.2008.04822.x 
Hilt, L.M., Cha, C.B., \& Nolen-Hoeksema, S. (2008). Nonsuicidal self-injury in young adolescent girls: Moderators of the distress-function relationship. Journal of Consulting and Clinical Psychology, 76, 63-71. doi:10.1037/0022-006X.76.1.63

Himber, J. (1994). Blood rituals: Self-cutting in female psychiatric inpatients. Psychotherapy: Theory, Research, Practice, Training, 31, 620-631. doi:10.1037/ 0033-3204.31.4.620

Hintikka, J., Tolmunen, T., Rissanen, M., Honkalampi, K., Kylmä, J., \& Laukkanen, E. (2009). Mental disorders in self-cutting adolescents. Journal of Adolescent Health, 44, 464-467. doi:10.1016/j.jadohealth.2008.10.003

Hjelmeland, H., \& Knizek, B.L. (2010). Why we need qualitative research in suicidology. Suicide and Life-Threatening Behavior, 40, 74-80. doi:10.1521/ suli.2010.40.1.74

Hodgson, S. (2004). Cutting through the silence: A sociological construction of selfinjury. Sociological Inquiry, 74, 162-179. doi:10.1111/j.1475-682X.2004.00085.x

Hofer, B. (2002). Personal epistemology as a psychological and educational construct: An introduction. In B. Hofer \& P. Pintrich, Ed., Personal epistemology: The psychology of beliefs about knowledge and knowing (pp. 3-15). Mahwah, NJ: Erlbaum.

Hoff, E.R., \& Muehlenkamp, J.J. (2009). Nonsuicidal self-injury in college students: The role of perfectionism and rumination. Suicide and Life-Threatening Behavior, 39, 576-587. doi:10.1521/suli.2009.39.6.576

Hofmann, S.G., \& Asmundson, G.J.G. (2008). Acceptance and mindfulness-based therapy: New wave or old hat ? Clinical Psychology Review, 28, 1-16. doi:10.1016/j.cpr.2007.09.003

Hollon, S.D., \& Kendall, P.C. (1980). Cognitive self-statements in depression: Development of an automatic thoughts questionnaire. Cognitive Therapy and Research, 4, 383-395. doi:10.1007/BF01178214

Howard, G.S. (1985). The role of values in the science of psychology. American Psychologist, 40, 255-265. doi:10.1037/0003-066X.40.3.255 
Husain, M.I., Waheed, W., \& Husain, N. (2006). Self-harm in British South Asian women: Psychosocial correlates and strategies for prevention. Annals of General Psychiatry, 5:7. doi:10.1186/1744-859X-5-7

Jacobson, C.M., Muehlenkamp, J.J., Miller, A.L., \& Turner, J.B. (2008). Psychiatric impairment among adolescents engaging in different types of deliberate selfharm. Journal of Clinical Child and Adolescent Psychology, 37, 363-375. doi:10.1080/15374410801955771

Janis, I.B., \& Nock, M.K. (2009). Are self-injurers impulsive?: Results from two behavioral laboratory studies. Psychiatry Research, 169, 261-267. doi:10.1016/ j.psychres.2008.06.041

Joiner, T. (2005). Why people die by suicide. Cambridge, Mass.: Harvard University Press.

Jorm, A.F., Kelly, C.M., \& Morgan, A.J. (2007). Participant distress in psychiatric research: A systematic review. Psychological Medicine, 37, 917-926. doi:10.1017/S0033291706009779

Joyce, P.R., McKenzie, J.M., Mulder, R.T., Luty, S.E., Sullivan, P.F., Miller, A.L., \& Kennedy, M.A. (2006). Genetic, developmental and personality correlates of self-mutilation in depressed patients. Australian and New Zealand Journal of Psychiatry, 40, 225-229. doi:10.1111/j.1440-1614.2006.01778.x

Jutengren, G., Kerr, M., \& Stattin, H. (2011). Adolescents' deliberate self-harm, interpersonal stress, and the moderating effects of self-regulation: A twowave longitudinal analysis. Journal of School Psychology, 49, 249-264. doi:10.1016/j.jsp.2010.11.001

Kamphuis, J.H., Ruyling, S.B., \& Reijntjes, A.H. (2007). Testing the emotion regulation hypothesis among self-injuring females: Evidence for differences across mood states. Journal of Nervous and Mental Disease, 195, 912-918. doi:10.1097/NMD.0b013e3181593d89 
Kam-shing, Y., Mei-yuk, N., \& Lam, I. (2003). A qualitative study of parental influence on and response to adolescents' self-cutting in Hong Kong. Families in Society, 84, 405-416.

Kassam-Adams, N., \& Newman, E. (2002). The reactions to research participation questionnaires for children and for parents (RRPQ-C and RRPQ-P). General Hospital Psychiatry, 24, 336-342.

Kassam-Adams, N., \& Newman, E. (2005). Child and parent reactions to participation in clinical research. General Hospital Psychiatry, 27, 29-35. doi:10.1016/j.genhosppsych.2004.08.007

Kearney, A.J. (2008). Understanding applied behavior analysis: An introduction to ABA for parents, teachers, and other professionals. London: Jessica Kingsley Publishers.

Kettlewell, C. (1999). Skin Game. New York: St. Martin's Griffin.

Kemperman, I., Russ, M.J., \& Shearin, E. (1997). Self-injurious behavior and mood regulation in borderline patients. Journal of Personality Disorders, 11, 146-157.

Kendall, P.C., Howard, B.L., \& Hays, R.C. (1989). Self-referent speech and psychopathology: The balance of positive and negative thinking. Cognitive Therapy and Research, 13, 583-598. doi:10.1007/BF01176069

Kenny, D.A. (1975). Cross-lagged panel correlation: A test for spuriousness. Psychological Bulletin, 82, 887-903. doi:10.1037/0033-2909.82.6.887

Kliem, S., Kröger, C., \& Kosfelder, J. (2010). Dialectical behavior therapy for borderline personality disorder: A meta-analysis using mixed-effects modeling. Journal of Consulting and Clinical Psychology, 78, 936 -951. doi:10.1037/a0021015

Kleindienst, N., Bohus, M., Ludäscher, P., Limberger, M.F., Kuenkele, K., EbnerPriemer, U.W.,...Schmahl, C. (2008). Motives for nonsuicidal self-injury among women with borderline personality disorder. Journal of Nervous and Mental Disease, 196, 230-236.

Kleinman, A. (1988). The illness narratives: Suffering, healing, and the human condition. New York: Basic Books. 
Klonsky, E.D. (2007). The functions of deliberate self-injury: A review of the evidence. Clinical Psychology Review, 27, 226-239. doi:10.1016/j.cpr.2006.08.002

Klonsky, E.D. (2009). The functions of self-injury in young adults who cut themselves: Clarifying the evidence for affect-regulation. Psychiatry Research, 166, 260-268. doi:10.1016/j.psychres.2008.02.008

Klonsky, E.D. (2011). Non-suicidal self-injury in United States adults: Prevalence, sociodemographics, topography and functions. Psychological Medicine, 41, 1981-1986. doi:10.1017/S0033291710002497

Klonsky, E.D., \& Glenn, C.R. (2009). Assessing the functions of non-suicidal selfinjury: Psychometric properties of the Inventory of Statements about Selfinjury (ISAS). Journal of Psychopathology and Behavioral Assessment, 31, 215-219. doi:10.1007/s10862-008-9107-z

Klonsky, E.D., \& Moyer, A. (2008). Childhood sexual abuse and non-suicidal selfinjury: Meta-analysis. British Journal of Psychiatry, 192, 166-170. doi:10.1192/bjp.bp.106.030650

Klonsky, E.D., \& Muehlenkamp, J.J. (2007). Self-injury: A research review for the practitioner. Journal of Clinical Psychology, 63, 1045-1056. doi:10.1002/jclp.20412

Klonsky, E.D., \& Olino, T.M. (2008). Identifying clinically distinct subgroups of selfinjurers among young adults: A latent class analysis. Journal of Consulting and Clinical Psychology, 76, 22-27. doi:10.1037/0022-006X.76.1.22

Kohlenberg, R.J., Tsai, M., \& Dougher, M.J. (1993). The dimensions of clinical behavior analysis. The Behavior Analyst, 16, 271-282.

Kokaliari, E., \& Berzoff, J. (2008). Nonsuicidal self-injury among nonclinical college women: Lessons from Foucault. Affilia, 23, 259-269. doi:10.1177/08861099 08319120

Kooiman, C.G., Spinhoven, P., \& Trijsburg, R.W. (2002). The assessment of alexithymia: A critical review of the literature and a psychometric study of the Toronto Alexithymia Scale-20. Journal of Psychosomatic Research, 53, 1083-1090. doi:10.1016/S0022-3999(02)00348-3 
Kraut, R., Olson, J., Banaji, M., Bruckman, A., Cohen, J., \& Couper, M. (2004). Psychological research online: Report of board of scientific affairs' advisory group on the conduct of research on the Internet. American Psychologist, 59, 105-117. doi: 10.1037/0003-066X.59.2.105

Kuhn, T. (1977). The essential tension. Chicago: University of Chicago Press.

Kumar, G., Pepe, D., \& Steer, R.A. (2004). Adolescent psychiatric inpatients' selfreported reasons for cutting themselves. The Journal of Nervous and Mental Disease, 192, 830-836. doi:10.1097/01.nmd.0000146737.18053.d2

Laraway, S., Snycerski, S., Michael, J., \& Poling, A. (2003). Motivating operations and terms to describe them: Some further refinements. Journal of Applied Behavior Analysis, 36, 407-414. doi:10.1901/jaba.2003.36-407

Lawson-Te Aho, K., \& Liu, J.H. (2010). Indigenous suicide and colonization: The legacy of violence and the necessity of self-determination. International Journal of Conflict and Violence, 4, 124-133. urn: nbn:de:0070-ijcv-20101108

Laye-Gindhu, A., \& Schonert-Reichl, K.A. (2005). Nonsuicidal self-harm among community adolescents: Understanding the "whats" and "whys" of selfharm. Journal of Youth and Adolescence, 34, 447-457. doi:10.1007/s10964-005$7262-\mathrm{z}$

Leenaars, A.A., De Wilde, E.J., Wenckstern, S., \& Kral, M. (2001). Suicide notes of adolescents: A life-span comparison. Canadian Journal of Behavioural Science, 33, 47-57. doi:10.1037/h0087127

Lewinsohn, P.M., Mermelstein, R., Alexander, D., \& MacPhillamy, D. (1983). Manual for the unpleasant events schedule: A scale for the measurement of aversive events. Mimeo: University of Oregon.

Lieb, K., Zanarini, M.C., Schmahl, C., Linehan, M.M., \& Bohus, M. (2004). Borderline personality disorder. Lancet, 364, 453-461.

Linehan, M.M. (1993a). Skills training manual for treating borderline personality disorder. New York: The Guildford Press. 
Linehan, M.M. (1993b). Cognitive-behavioral treatment of borderline personality disorder. New York: The Guildford Press.

Linehan, M.M., Comtois, K.A., Brown, M.Z., Heard, H.L., \& Wagner, A. (2006). Suicide Attempt Self-Injury Interview (SASII): Development, reliability, and validity of a scale to assess suicide attempts and intentional self-injury. Psychological Assessment, 18, 303-312. doi:10.1037/1040-3590.18.3.303

Lloyd-Richardson, E.E., Perrine, N., Dierker, L., \& Kelley, M.L. (2007). Characteristics and functions of non-suicidal self-injury in a community sample of adolescents. Psychological Medicine, 37, 1183-1192. doi:10.1017/ S003329170700027X

Lovibond, S.H., \& Lovibond, P.F. (1995). Manual for the Depression Anxiety Stress Scales (2 ${ }^{\text {nd }}$ ed.). Sydney: Psychology Foundation.

Lucassen, M.F.G., Merry, S.N., Robinson, E.M., Denny, S., Clark, T., Ameratunga, S.,...Rossen, F.V. (2011). Sexual attraction, depression, self-harm, suicidality and help-seeking behaviour in New Zealand secondary school students. Australian and New Zealand Journal of Psychiatry, 45, 376-383. doi:10.3109/ 00048674.2011 .559635

Lundh, L.G., Karim, J., \& Quilisch, E. (2007). Deliberate self-harm in 15-year-old adolescents: A pilot study with a modified version of the Deliberate SelfHarm Inventory. Scandinavian Journal of Psychology, 48, 33-41. doi:10.1111/ j.1467-9450.2006.00567.x

Lynch, T.R., Chapman, A.L., Rosenthal, M.Z., Kuo, J.R., \& Linehan, M.M. (2006). Mechanisms of change in dialectical behavior therapy: Theoretical and empirical observations. Journal of Clinical Psychology, 62, 459-480. doi:10.1002/ jclp.20243

Mackinnon, D.P., \& Dwyer, J.H. (1993). Estimating mediated effects in prevention studies. Evaluation Review, 17, 144-158. doi:10.1177/0193841X9301700202 
MacLaren, V.V., \& Best, L.A. (2010). Nonsuicidal self-injury, potentially addictive behaviors, and the Five Factor Model in undergraduates. Personality and Individual Differences, 49, 521-525. doi:10.1016/j.paid.2010.05.019

Maniglio, R. (2011). The role of child sexual abuse in the etiology of suicide and nonsuicidal self-injury. Acta Psychiatrica Scandinavica, 124, 30-41. doi:10.1111/ j.1600-0447.2010.01612.x

Martens, M.P., \& Haase, R.F. (2006). Advanced applications of structural equation modeling in counseling psychology research. The Counseling Psychologist, 34, 878-911. doi:10.1177/0011000005283395

Matthews, P.C. (1968). Epidemic self-injury in an adolescent unit. International Journal of Social Psychiatry, 14, 125-133. doi:10.1177/002076406801400206

McGill, P. (1999). Establishing operations: Implications for the assessment, treatment, and prevention of problem behavior. Journal of Applied Behavior Analysis, 32, 393-418. doi:10.1901/jaba.1999.32-393

McMullin, E. (1983). Values in science. In P. D. Asquith \& T. Nickles (Eds.), Proceedings of the 1982 Philosophy of Science Association (Vol. 2, pp. 3-23). East Lansing, MI: Philosophy of Science Association.

Menninger, K.A. (1935). A psychoanalytic study of the significance of selfmutilations. Psychoanalytic Quarterly, 4, 408-466.

Messer, J.M., \& Fremouw, W.J. (2008). A critical review of explanatory models for self-mutilating behaviors in adolescents. Clinical Psychology Review, 28, 162178. doi:10.1016/j.cpr.2007.04.006

Michael, J. (1993). Establishing operations. The Behavior Analyst, 16, 191-206.

Miller, A.L., \& Smith, H.L. (2008). Adolescent non-suicidal self-injurious behavior: The latest epidemic to assess and treat. Applied and Preventive Psychology, 12, 178-188. doi:10.1016/j.appsy.2008.05.003

Miltenberger, R.G. (2004). Behavior modification: Principles and procedures ( $3^{\text {rd }}$ ed.). Pacific Grove, CA: Wadsworth. 
Miltenberger, R.G. (2005). The role of automatic negative reinforcement in clinical problems. International Journal of Behavioral Consultation and Therapy, 1, 1-11.

Ministry of Health. (2010). Suicide Facts: Deaths and intentional self-harm hospitalisations 2008. Wellington: Ministry of Health. Retrieved from http://www.moh.govt. nz/moh.nsf/pagesmh/10482/\$File/suicide-facts-2008-dec2010.pdf

Moffitt, T.E. (1993). Adolescence-limited and life-course-persistent antisocial behavior: A developmental taxonomy. Psychological Review, 100, 674-701. doi:10.1037/0033-295X.100.4.674

Muehlenkamp, J.J. (2005). Self-injurious behavior as a separate clinical syndrome. American Journal of Orthopsychiatry, 75, 324-333. doi:10.1037/0002-9432.75.2.324

Muehlenkamp, J.J., Engel, S.G., Wadeson, A., Crosby, R.D., Wonderlich, S.A., Simonich, H., \& Mitchell, J.E. (2009). Emotional states preceding and following acts of non-suicidal self-injury in bulimia nervosa patients. Behaviour Research and Therapy, 47, 83-87. doi:10.1016/j.brat.2008.10.011

Muehlenkamp, J.J., \& Gutierrez, P.M. (2004). An investigation of differences between self-injurious behavior and suicide attempts in a sample of adolescents. Suicide and Life-Threatening Behavior, 34, 12-23.

Muehlenkamp, J.J., \& Gutierrez, P.M. (2007). Risk for suicide attempts among adolescents who engage in non-suicidal self-injury. Archives of Suicide Research, 11, 69-82. doi:10.1080/13811110600992902

Muehlenkamp, J.J., Kerr, P.L., Bradley, A.R., \& Adams Larsen, M. (2010). Abuse subtypes and nonsuicidal self-injury: Preliminary evidence of complex emotion regulation patterns. Journal of Nervous and Mental Disease, 198, 258263. doi:10.1097/NMD.0b013e3181d612ab

Muehlenkamp, J.J., Williams, K.L., Gutierrez, P.M., \& Claes, L. (2009). Rates of nonsuicidal self-injury in high school students across five years. Archives of Suicide Research, 13, 317-329. doi:10.1080/13811110903266368 
Najmi, S., Wegner, D.M., \& Nock, M.K. (2007). Thought suppression and selfinjurious thoughts and behaviors. Behaviour Research and Therapy, 45, 19571965. doi:10.1016/j.brat.2006.09.014

National Institute for Health and Clinical Excellence. (2004). Self-harm: The short-term physical and psychological management and secondary prevention of self-harm in primary and secondary care (CG16). London: National Institute for Health and Clinical Excellence.

National Self-harm Network (2000). Cutting the risk: Self-harm, self-care $\mathcal{E}$ risk reduction. London: The National Self-harm Network.

Nelson-Gray, R.O., \& Farmer, R.F. (1999). Behavioral assessment of personality disorders. Behaviour Research and Therapy, 37, 347-368. doi:10.1016/S00057967(98)00142-9

Netemeyer, R.G., Williamson, D.A, Burton, S., Biswas, D., Jindal, S., Landreth, S.,...Primeaux, S. (2002). Psychometric properties of shortened versions of the Automatic Thoughts Questionnaire. Educational and Psychological Measurement, 62, 111-129. doi:10.1177/0013164402062001008

Niedtfeld, I., Schulze, L., Kirsch, P., Herpertz, S.C., Bohus, M., \& Schmahl, C. (2010). Affect regulation and pain in borderline personality disorder: A possible link to the understanding of self-injury. Biological Psychiatry, 68, 383-391. doi:10.1016/j.biopsych.2010.04.015

Nixon, M.K., Cloutier, P.F., \& Aggarwal, S. (2002). Affect regulation and addictive aspects of repetitive self-injury in hospitalized adolescents. Journal of the American Academy of Child \& Adolescent Psychiatry, 41, 1333-1341. doi:10.1097/01.CHI.0000024844.60748.C6

Nixon, M.K., Cloutier, P., \& Jansson, S.M. (2008). Nonsuicidal self-harm in youth: A population-based survey. Canadian Medical Association Journal, 178, 306-312. doi:10.1503/cmaj.061693 
Nock, M.K. (2008). Actions speak louder than words: An elaborated theoretical model of the social functions of self-injury and other harmful behaviors. Applied and Preventive Psychology, 12, 159-168. doi:10.1016/j.appsy.2008.05.002

Nock, M.K. (2009). Why do people hurt themselves? New insights into the nature and functions of self-injury. Current Directions in Psychological Science, 18, 7883. doi:10.1111/j.1467-8721.2009.01613.x

Nock, M.K. (2010). Self-injury. Annual Review of Clinical Psychology, 6, 339-363. doi:10.1146/annurev.clinpsy.121208.131258

Nock, M.K., Joiner, T.E., Gordon, K.H. Lloyd-Richardson, E., \& Prinstein, M.J. (2006). Non-suicidal self-injury among adolescents: Diagnostic correlates and relation to suicide attempts. Psychiatry Research, 144, 65-72. doi:10.1016/j.psychres.2006. 05.010

Nock, M.K., \& Mendes, W.B. (2008). Physiological arousal, distress tolerance, and social problem-solving deficits among adolescent self-injurers. Journal of Consulting and Clinical Psychology, 76, 28-38. doi: 10.1037/0022-006X.76.1.28

Nock, M.K., \& Prinstein, M.J. (2004). A functional approach to the assessment of selfmutilative behavior. Journal of Consulting and Clinical Psychology, 72, 885-890. doi:10.1037/0022-006X.72.5.885

Nock, M.K., \& Prinstein, M.J. (2005). Contextual features and behavioral functions of self-mutilation among adolescents. Journal of Abnormal Psychology, 114, 140146. doi:10.1037/0021-843X.114.1.140

Nock, M.K., Prinstein, M.J., \& Sterba, S.K. (2009). Revealing the form and function of self-injurious thoughts and behaviors: A real-time ecological assessment study among adolescents and young adults. Journal of Abnormal Psychology, 118, 816-827. doi:10.1037/a0016948

Nock, M.K., Wedig, M.M., Holmberg, E.B., \& Hooley, J.M. (2008). The emotion reactivity scale: Development, evaluation, and relation to self-injurious thoughts and behaviors. Behavior Therapy, 39, 107-116. doi:10.1016/ j.beth. 2007.05.005 
Norusis, M.J. (2010). PASW statistics 18 statistical procedures companion. Upper Saddle River, NJ: Prentice Hall.

O'Connor, R.C., Rasmussen, S., Miles, J., \& Hawton, K. (2009). Self-harm in adolescents: Self-report survey in schools in Scotland. The British Journal of Psychiatry, 194, 68-72. doi:10.1192/bjp.bp.107.047704

O'Connor, R.C., Sheehy, N.P., \& O'Connor, D.B. (1999). A thematic analysis of suicide notes. Crisis, 20, 106-114. doi:10.1027//0227-5910.20.3.106

Olson, C.L. (1974). Comparative robustness of six tests in multivariate analysis of variance. Journal of the American Statistical Association, 69, 894-908.

Osman, A., Bagge, C.L., Gutierrez, P.M., Konick, L.C., Kooper, B.A., \& Barrios, F.X. (2001). The Suicidal Behaviors Questionnaire-Revised (SBQ-R): Validation with clinical and nonclinical samples. Assessment, 8, 443-454. doi:10.1177/107319110100800409

Osuch, E.A., Noll, J.G., \& Putnam, F.W. (1999). The motivations for self-injury in psychiatric inpatients. Psychiatry, 62, 334-346.

Oyefeso, A., Brown, S., Chiang, Y., \& Clancy, C. (2008). Self-injurious behaviour, traumatic life events and alexithymia among treatment-seeking opiate addicts: Prevalence, pattern and correlates. Drug and Alcohol Dependence, 98, 227-234. doi:10.1016/j.drugalcdep.2008.06.004

Paivio, S.C., \& McCulloch, C.R. (2004). Alexithymia as a mediator between childhood trauma and self-injurious behaviors. Child Abuse \& Neglect, 28, 339354. doi:10.1016/j.chiabu.2003.11.018

Pattison, E.M., \& Kahan, J. (1983). The deliberate self-harm syndrome. American Journal of Psychiatry, 140, 867-872.

Pavlov, I. (1927). Conditioned reflexes. London: Oxford University Press.

Pembroke, L.R. (Ed.). (1996a). Self-harm: Perspectives from personal experience (Rev. ed.). London: Survivors Speak Out.

Pembroke, L.R. (1996b). Introduction. In L.R. Pembroke (Ed.), Self-harm: Perspectives from personal experience (Rev. ed., pp. 1-4). London: Survivors Speak Out. 
Pembroke, L. (1998). Self-harm: A personal story. Mental Health Practice, 2, 20-24.

Pembroke, L. (2000). Introduction. In the National Self-harm Network, Cutting the risk: Self-harm, self-care E risk reduction (pp. 5-7). London: The National Selfharm Network.

Penrose-Wall, J., Farris, Z., \& Berkery, P. (2005). Self-harm: New Zealand treatment guide for consumers and carers. Wellington: Royal Australian and New Zealand College of Psychiatrists. Retrieved from http://www.ranzcp.org/ images/stories/ranzcp-attachments/Resources/Publications/CPG/NZ_ Versions/NZ_Self_harm.pdf

Plener, P.L., Libal, G., Keller, F., Fegert, J.M., \& Muehlenkamp, J.J. (2009). An international comparison of adolescent non-suicidal self-injury (NSSI) and suicide attempts: Germany and the USA. Psychological Medicine, 39, 1549-1558. doi:10.1017/S0033291708005114

Polk, E., \& Liss, M. (2007). Psychological characteristics of self-injurious behavior. Personality and Individual Differences, 43, 567-577. doi:10.1016/j.paid.2007.01.003

Polk, E., \& Liss, M. (2009). Exploring the motivations behind self-injury. Counselling Psychology Quarterly, 22, 233-241. doi:10.1080/09515070903216911

Prinstein, M.J. (2008). Introduction to the special section on suicide and nonsuicidal self-injury: A review of unique challenges and important directions for selfinjury science. Journal of Consulting and Clinical Psychology, 76, 1-8. doi:10.1037/ 0022-006X.76.1.1

Rassin, E. (2003). The White Bear Suppression Inventory (WBSI) focuses on failing suppression attempts. European Journal of Personality, 17, 285-298. doi:10.1002/ per.478

Reynolds, S.K., Lindenboim, N., Comtois, K.A., Murray, A., \& Linehan, M.M. (2006). Risky assessments: Participant suicidality and distress associated with research assessments in a treatment study of suicidal behavior. Suicide and Life-threatening Behavior, 36, 19-34. doi:10.1521/suli.2006.36.1.19 
Rissanen, M., Kylmä, J., \& Laukkanen, E. (2008). Descriptions of self-mutilation among Finnish adolescents: A qualitative descriptive inquiry. Issues in Mental Health Nursing, 29, 145-163. doi:10.1080/01612840701792597

Robinson, J., Pan Yuen, H., Martin, C., Hughes, A., Baksheev, G.N., Dodd, S.,...Yung, A.R. (2011). Does screening high school students for psychological distress, deliberate self-harm, or suicidal ideation cause distress - and is it acceptable? An Australian-based study. Crisis, 32, 254-263. doi:10.1027/02275910/a000087

Rooney, P. (1992). On values in science: Is the epistemic/non-epistemic distinction useful? PSA: Proceedings of the Biennial Meeting of the Philosophy of Science Association (vol. 1, pp. 13-22). Chicago: The University of Chicago Press.

Rosen, P.M., \& Walsh, B.W. (1989). Patterns of contagion in self-mutilation epidemics. The American Journal of Psychiatry, 146, 656-658.

Rosenthal, M.Z., Gratz, K.L., Kosson, D.S., Cheavens, J.S., Lejuez, C.W., \& Lynch, T.R. (2008). Borderline personality disorder and emotional responding: A review of the research literature. Clinical Psychology Review, 28, 75-91. doi:10.1016/j.cpr.2007.04.001

Ross, M. (1996). Maggy Ross. In L.R. Pembroke (Ed.), Self-harm: Perspectives from personal experience (Rev. ed., pp. 13-15). London: Survivors Speak Out.

Ross, S., \& Heath, N. (2002). A study of the frequency of self-mutilation in a community sample of adolescents. Journal of Youth and Adolescence, 31, 67-77.

Ross, S., Heath, N.L., \& Toste, J.R. (2009). Non-suicidal self-injury and eating pathology in high school students. American Journal of Orthopsychiatry, 79, 8392. doi:10.1037/a0014826

Rowland, M. (2011). Acceptance and commitment therapy for non-suicidal self-injury among adolescents (Unpublished doctoral dissertation). The Chicago School of Professional Psychology, United States. 
Russ, M.J., Roth, S.D., Lerman, A., Kakuma, T., Harrison, K., Shindledecker, R.D.,...\& Mattis, S. (1992). Pain perception in self-injurious patients with borderline personality disorder. Biological Psychiatry, 32, 501-511.

Russell, G., Moss, D., \& Miller, J. (2010). Appalling and appealing: A qualitative study of the character of men's self-harm. Psychology and Psychotherapy, 83, 91109. doi:10.1348/147608309X466826

Savin-Williams, R.C., \& Ream, G.L. (2007). Prevalence and stability of sexual orientation components during adolescence and young adulthood. Archives of Sexual Behavior, 36, 385-394. doi:10.1007/s10508-006-9088-5

Schmidtke, A., Bille-Brahe, U., De Leo, D., Kerkhof, A., Bjerke, T., Crepef, P., Haring, C.,...Sampaio-Faria, J.G. (1996). Attempted suicide in Europe: Rates, trends and sociodemographic characteristics of suicide attempters during the period 1989-1992. Results of the WHO/EURO Multicentre Study on Parasuicide. Acta Psychiatrica Scandinavica, 93, 327-338. doi:10.1111/j.1600-0447.1996.tb10656.x

Schneidman, E.S. (1996). The suicidal mind. Oxford: Oxford University Press.

Schommer-Aikins, M. (2002). An evolving theoretical framework for an epistemological belief system. In B.K. Hofer, \& P.R. Pintrich (Eds.). Personal epistemology: The psychology of beliefs about knowledge and knowing (pp. 103-118). Mahwah, NJ: Lawrence Erlbaum Associates.

Schoppmann, S., Schröck, R., Schnepp, W., \& Büscher, A. (2007). ‘Then I just showed her my arms ...' Bodily sensations in moments of alienation related to selfinjurious behaviour. A hermeneutic phenomenological study. Journal of Psychiatric and Mental Health Nursing, 14, 587-597. doi:10.1111/j.1365-2850. 2007.01150.x

Shaw, S.N. (2002). Shifting conversations on girls' and women's self-injury: An analysis of the clinical literature in historical context. Feminism E Psychology, 12, 191-219. doi:10.1177/0959353502012002010 
Shearer, S.L. (1994). Phenomenology of self-injury among inpatient women with borderline personality disorder. Journal of Nervous and Mental Disease, 182, $524-526$.

Sheldon, M. (2001). Psychiatric assessment in remote Aboriginal communities. Australian and New Zealand Journal of Psychiatry, 35, 435-442.

Shenk, C.E., Noll, J.G., \& Cassarly, J.A. (2010). A multiple mediational test of the relationship between childhood maltreatment and non-suicidal self-injury. Journal of Youth and Adolescence, 39, 335-342. doi:10.1007/s10964-009-9456-2

Sher, L., \& Stanley, B.H. (2008). The role of endogenous opioids in the pathophysiology of self-injurious and suicidal behavior. Archives of Suicide Research, 12, 299-308. doi:10.1080/13811110802324748

Shorter, E. (1987). The first great increase in anorexia nervosa. Journal of Social History, 21, 69-96.

Skegg, K., Nada-Raja, S., Dickson, N., Paul, C., \& Williams, S. (2003). Sexual orientation and self-harm in men and women. American Journal of Psychiatry, 160, 541-546. doi: 10.1176/appi/ajp.160.3.541

Skinner, E.A., Edge, K., Altman, J., \& Sherwood, H. (2003). Searching for the structure of coping: A review and critique of category systems for classifying ways of coping. Psychological Bulletin, 129, 216-269. doi:10.1037/00332909.129.2.216

Skodol, A.E., \& Bender, D.S. (2003). Why are women diagnosed borderline more than men? Psychiatric Quarterly, 74, 349-360.

Stallman, H.M. (2010). Psychological distress in university students: A comparison with general population data. Australian Psychologist, 45, 249-257. doi:10.1080/ 00050067.2010.482109

Stanley, B., Brodsky, B., Nelson, J.D., \& Dulit, R. (2007). Brief dialectical behavior therapy (DBT-B) for suicidal behavior and non-suicidal self injury. Archives of Suicide Research, 11, 337-341. doi:10.1080/13811110701542069 
Stickney, M.I., \& Miltenberger, R.G. (1999). Evaluating direct and indirect measures for the functional assessment of binge eating. International Journal of Eating Disorders, 26, 195-204.

Strong, M. (2000). A bright red scream: Self-mutilation and the language of pain. London: Virago Press.

Sturmey, P., Ward-Horner, J., Marroquin, M., \& Doran., E. (2007a). Structural and functional approaches to psychopathology and case formulation. In P. Sturmey (Ed.), Functional analysis in clinical treatment (pp. 1-21). Burlington, MA: Academic Press.

Sturmey, P., Ward-Horner, J., Marroquin, M., \& Doran., E. (2007b). Operant and respondent behavior. In P. Sturmey (Ed.), Functional analysis in clinical treatment (pp. 23-50). Burlington, MA: Academic Press.

Sturmey, P., Ward-Horner, J., Marroquin, M., \& Doran, E. (2007c). Advanced concepts and methods of intervention in behavioral approaches to psychopathology. In P. Sturmey (Ed.), Functional analysis in clinical treatment (pp. 51-64). Burlington, MA: Academic Press.

Suyemoto, K.L. (1998). The functions of self-mutilation. Clinical Psychology Review, 18, 531-554. doi:10.1016/S0272-7358(97)00105-0

Swann, W.B., Hixon, J.G., Stein-Seroussi, A., \& Gilbert, D.T. (1990). The fleeting gleam of praise: Cognitive processes underlying behavioral reactions to selfrelevant feedback. Journal of Personality and Social Psychology, 59, 17-26. doi:10.1037/0022-3514.59.1.17

Swannell, S., Martin., G., Scott, J., Gibbons, M., \& Gifford, S. (2008). Motivations for self-injury in an adolescent inpatient population: Development of a self-report measure. Australasian Psychiatry, 16, 98-103. doi:10.1080./10398560701636955

Swenson, L.P., Spirito, A., Dyl, J., Kittler, J., \& Hunt, J.I. (2008). Psychiatric correlates of nonsuicidal cutting behaviors in an adolescent inpatient sample. Child Psychiatry and Human Development, 39, 427-438. doi:10.1007/s10578-008-0100-2 
Tabachnick, B.G., \& Fidell, L.S. (2007). Using multivariate statistics (5 ${ }^{\text {th }}$ ed.). Boston, MA: Pearson.

Taylor, B. (2003). Exploring the perspectives of men who self-harm. Learning in Health and Social Care, 2, 83-91. doi:10.1046/j.1473-6861.2003.00042.x

Tourangeau, R. (2000). Remembering what happened: Memory errors and survey reports. In A.A. Stone, J.S. Turkkan, C.A. Bachrach, J.B. Jobe, \& H.S. Kurtzman (Eds.), The science of self-report: Implications for research and practice (pp. 29-47). Mahwah, NJ: Lawrence Erlbaum Associates.

Turp, M. (2003). Hidden self-harm: Narratives from psychotherapy. London: Jessica Kingsley Publishers.

Vega, V. (2007). Comes the darkness, comes the light: A memoir of cutting, healing, and hope. New York: Amacon.

Walsh, B.W. (2006). Treating self-injury: A practical guide. New York: Guilford Press. Waternaux, C.M. (1976). Asymptotic distribution of the sample roots for a nonnormal population. Biometrika, 63, 639-645. doi:10.1093/biomet/63.3.639 Watson, D., \& Clark, L.A. (1994). The PANAS-X Manual for the Positive and Negative Affect Schedule: Expanded Form. Retrieved from www.psychology.uiowa.edu/ faculty/clark/panas-x.pdf.

Watson, D., \& Tellegen, A. (1985). Toward a consensual structure of mood. Psychological Bulletin, 98, 219-235. doi:10.1037/0033-2909.98.2.219

Watters, E. (2010). Crazy like us: The globalization of the American psyche. New York: Free Press.

Wegner, D.M., \& Zanakos, S. (1994). Chronic thought suppression. Journal of Personality, 62, 615-640. doi:10.1111/j.1467-6494.1994.tb00311.x

Weierich, M.R., \& Nock, M.K. (2008). Posttraumatic stress symptoms mediate the relation between childhood sexual abuse and nonsuicidal self-injury. Journal of Consulting and Clinical Psychology, 76, 39-44. doi:10.1037/0022-006X.76.1.39 
Weismoore, J.T., \& Esposito-Smythers, C. (2010). The role of cognitive distortion in the relationship between abuse, assault, and non-suicidal self-injury. Journal of Youth and Adolescence, 39, 281-290. doi:10.1007/s10964-009-9452-6

Welch, S.S., Linehan, M.M., Sylvers, P., Chittams, J., \& Rizvi, S.L. (2008). Emotional responses to self-injury imagery among adults with borderline personality disorder. Journal of Consulting and Clinical Psychology, 76, 45-51. doi:10.1037/0022-006X.76.1.45

Westbrook, D., Kennerley, H., \& Kirk, J. (2007). An introduction to cognitive behaviour therapy: Skills and applications. London: Sage.

Whitlock, J., Eckenrode, J., \& Silverman, D. (2006). Self-injurious behaviors in a college population. Pediatrics, 117, 1939-1948. doi:10.1542/peds.2005-2543

Whitlock, J., \& Knox, K.L. (2007). The relationship between self-injurious behavior and suicide in a young adult population. Archives of Pediatrics $\mathcal{E}$ Adolescent Medicine, 161, 634-640. doi:10.1001/archpedi.161.7.634

Whitlock, J., Lader, W., \& Conterio, K. (2007). The internet and self-injury: What psychotherapists should know. Journal of Clinical Psychology, 63, 1135-1143. doi:10.1002/jclp.20420

Whitlock, J., Muehlenkamp, J., \& Eckenrode, J. (2008). Variation in nonsuicidal selfinjury: Identification and features of latent classes in a college population of emerging adults. Journal of Clinical Child and Adolescent Psychology, 37, 725-735. doi:10.1080/15374410802359734

Wichstrøm, L. (2009). Predictors of non-suicidal self-injury versus attempted suicide: Similar or different? Archives of Suicide Research, 13, 105-122. doi:10.1080/ 13811110902834992

Wilson, C. (1999). Ethnicity and deliberate self-injury: A review of the literature. In N. Robertson (Ed.), Māori and psychology: Research and practice - The proceedings of a symposium sponsored by the Māori and Psychology Research Unit. Hamilton: Māori \& Psychology Research Unit. 
Wykes, T., \& Callard, F. (2010). Diagnosis, diagnosis, diagnosis: Towards DSM-5. Journal of Mental Health, 19, 301-304. doi:10.3109/09638237.2010.494189

Yates, T.M. (2004). The developmental psychopathology of self-injurious behavior: Compensatory regulation in posttraumatic adaptation. Clinical Psychology Review, 24, 35-74. doi:10.1016/j.cpr.2003.10.001

Yates, T.M., Carlson, E.A, \& Egeland, B. (2008). A prospective study of child maltreatment and self-injurious behavior in a community sample. Development and Psychopathology, 20, 651-671. doi:10.1017/S0954579408000321

Yip, K. (2005). A multi-dimensional perspective of adolescents' self-cutting. Child and Adolescent Mental Health, 10, 80-86. doi:10.1111/j.1475-3588.2005.00122.x

Zahl, D.L., \& Hawton, K. (2004). Repetition of deliberate self-harm and subsequent suicide risk: Long-term follow-up study of 11,583 patients. The British Journal of Psychiatry, 185, 70-75.

Zanarini, M.C., Laudate, C.S., Frankenburg, F.R., Reich, D.B., \& Fitzmaurice, G. (2011). Predictors of self-mutilation in patients with borderline personality disorder: A 10-year follow-up study. Journal of Psychiatric Research, 45, 823-828. doi:10.1016/j.jpsychires.2010.10.015

Zinck, A. (2008). Self-referential emotions. Consciousness and Cognition, 17, 496-505. doi:10.1016/j.concog.2008.03.014

Zlotnick, C., Shea, M.T., Pearlstein, T., Simpson, E., Costello, E., \& Begin, A. (1996). The relationship between dissociative symptoms, alexithymia, impulsivity, sexual abuse, and self-mutilation. Comprehensive Psychiatry, 37, 12-16. 


\title{
Exploring the experiences of adolescents and young adults who engage in non-suicidal deliberate self-harm behaviours
}

\author{
Principle Investigator: $\quad$ Robyn Langlands, PhD Student \\ School of Psychology \\ PO Box 600 \\ Victoria University of Wellington \\ Ph: (04) 4635233 extension 8605 \\ Email: robyn.langlands@vuw.ac.nz \\ Supervisor: \\ Dr Marc Wilson \\ School of Psychology \\ PO Box 600 \\ Victoria University of Wellington \\ Ph: (04) 4635225 \\ Email: marc.wilson@vuw.ac.nz
}

\section{Introduction}

You are invited to take part in the following study. The study will involve the following three phases:

1) Completing a questionnaire about the different types of non-suicidal deliberate self-harm behaviours you may have engaged in, as well as how often and severe these behaviours have been.

2) Potentially participating in one or two interviews to discuss deliberate self-harm behaviours, depending on the results of your questionnaire. The interview(s) will last approximately one to two hours.

3) Completing a questionnaire (if you have taken part in the interview(s)) about participating in this research study. This questionnaire will include the opportunity to provide suggestions for future research on the topic of deliberate self-harm.

Your participation is entirely voluntary (your choice). You do not have to take part in this study, and if you do choose not to take part, this will not affect any future care or treatment. If you do agree to take part, you are free to withdraw from the study at any time, without having to give a reason and this will in no way affect your continuing health care. If you do withdraw from the study before completing the questionnaire(s) or interview(s), all the data that has been collected in relation to you (e.g. completed questionnaires or interview transcripts) will be immediately destroyed. You do not have to answer all the questions, and you may stop the interview at any time. Participation in this study will be stopped should the Principal Investigator feel it is not in your best interest to continue. You may have support from a friend, family or whanau member to help you understand the risks and/or benefits of this study and any other explanation you may require. This study has received ethical approval from the Multiregion Ethics Committee which reviews National and Multi-regional studies. 
Deliberate self-harm is a seldom investigated topic in New Zealand and as a result, very little is known about New Zealanders' experiences of self-harm. The purpose of this study is to explore what kinds of thoughts, feelings and events may lead adolescents and young adults to engage in deliberate self-harm, and the purpose(s) that self-harm fulfils for them. This study is being conducted as part of a PhD thesis. The Principal Investigator is interested in learning about your experiences of deliberate self-harm from your perspective. The study will not involve you participating in any form of therapy. The ultimate goal of the thesis is to identify factors that trigger and maintain non-suicidal self-harm behaviours in order to improve mental health treatments for people who self-harm.

\section{Who is being asked to participate in this study?}

Individuals will be asked to participate in the study if they are between the ages of 16 and 34, can speak English fluently, and have engaged in deliberate self-harm within the past 12 months. Individuals will be excluded if they have an intellectual disability, have only engaged in deliberate self-harm during episodes of mania or psychosis, or if they are currently experiencing a manic or psychotic episode.

\section{How were participants selected for this study, and who selected them?}

There are two groups of participants involved in this study-individuals who are engaged in public mental health and counselling services, and individuals recruited from the community. In regards to individuals from mental health and counselling services, the Principal Investigator approached mental health clinicians to inform them about the study. These clinicians were asked to pass on information about the study to any of their clients, between the ages of 16 to 34 years old, who have engaged in deliberate self-harm behaviour(s) within the past 12 months and who may be interested in participating in the study. To recruit participants from the community, the Principal Investigator placed advertisements about the study in community newspapers, newsletters, and venues inviting individuals who have engaged in deliberate self-harm behaviour(s) to contact her if they would like to participate in the study.

\section{How many participants will be involved?}

The Principal Investigator intends to interview at least 12-15 participants.

\section{Where will the interviews be conducted?}

The interviews will take place either at the mental health clinic or counselling service that the participant attends (if appropriate), or at the School of Psychology at Victoria University's Kelburn Campus.

\section{What will happen during the study?}

If you agree to participate in this study, the following will take place:

1. Robyn Langlands, the Principal Investigator, will send you a questionnaire to complete about deliberate self-harm behaviour(s). 
2. Depending on the results of your questionnaire, you may be asked to participate in an interview to discuss deliberate self-harm behaviours. You will be given a list of questions that will be asked during the interview to enable you to decide whether you will be comfortable discussing such topics.

3. If you decide you would like to participate in the interview, Robyn will arrange a suitable time and place to meet you for the interview. You are welcome to bring a support person with you to the interview.

4. You will be asked to sign a consent form to show that you understand what the study is about and that you agree to participate. If you have a mental health clinician, by giving your consent you will also be giving permission for Robyn to contact your mental health clinician to inform them that you are taking part in the study and to confirm what particular mental health diagnoses you have been given. The information that you share with Robyn during the interview will not be discussed with your clinician unless she believes that you are danger of harming yourself or someone else, in which case she is legally required to break confidentiality.

5. During the interview, Robyn will ask you a number of questions about your self-harm behaviour(s). This interview will take approximately 1-2 hours and will be audio-taped for later transcription. If you or Robyn does not believe that you have covered all the necessary information within the interview, you will be given the opportunity to participate in a second interview. The tapes and transcripts from the interview(s) will be stored in a locked filing cabinet following the study.

6. Robyn will contact you a day or two after the interview(s) to ask whether the study has raised any questions for you that you would like answered.

7. Within 2 weeks of completing the interview(s), you will receive a questionnaire to fill in about your experiences of participating in a research study on deliberate self-harm. This questionnaire will give you the opportunity to provide suggestions about how this type of research can be improved.

\section{What about privacy?}

No material which could personally identify you will be used in any reports on this study. The information will be used as part of the researcher's $\mathrm{PhD}$ thesis and may be published in an academic journal or presented at conferences. In any publication or presentation, information will be provided in such a way that you cannot be identified. Some publications require that the data described is made available to competent professionals. If the data is requested by other professionals, it will be provided in such a way that no-one will be able to identify individual participants.

\section{Are there any risks?}

Deliberate self-harm can be a very difficult topic to talk about and there is a risk that some of the questions asked may bring up past memories or feelings that are unpleasant or distressing. If you do become distressed during the interview, you can stop the interview at any time. If you find that you feel upset after the interview, you are welcome to contact Robyn Langlands. Robyn will also explore options for further support with you at the end of the interview.

\section{What are the benefits?}

There is no guarantee that you will receive any benefits from this project. However, this project does have the potential to lead to better mental health care and support for people who engage in deliberate self-harm. 


\section{Will I be paid to participate in this project?}

You will be given a movie voucher to the value of one movie ticket for completing the initial questionnaire. If you participate in the interview, you will be given the choice of a petrol voucher, Farmer's voucher or The Warehouse voucher to the value of $\$ 30$ to reimburse you for your time and travel expenses. Finally, if you complete the questionnaire on research participation, you will be given a movie voucher to the value of one movie ticket.

\section{What will happen at the end of the study?}

You will be asked whether you would like to be sent a transcript of your interview(s) once the data has been transcribed. You will also be given the option of receiving a brief summary of the common themes that arose from the interviews once the data has been transcribed and analysed.

\section{Where can I get more information about the study?}

If you would like further information about this project, please contact Robyn Langlands, email: robyn.langlands@vuw.ac.nz, phone: (04) 4635233 extension 8605. If you do phone Robyn and you are directed through to an answer phone, please leave your name and number, and she will call you back as soon as possible. The answer phone is private and Robyn will be the only person who has access to the messages left on the phone.

\section{What if I have concerns about the project?}

If you have any questions or concerns about your rights as a participant in this research study, you can contact an independent health and disability advocate.

This is a free service provided under the Health and Disability Commissioner Act.

Telephone (NZwide):

Free Fax (NZ wide):

Email (NZ wide):
0800555050

080027877678 (0800 2 SUPPORT)

advocacy@hdc.org.nz

\section{Compensation}

In the unlikely event of a physical injury as a result of your participation in this study, you may be covered by ACC under the Injury Prevention, Rehabilitation and Compensation Act. ACC cover is not automatic and your case will need to be assessed by ACC according to the provisions of the 2002 Injury Prevention Rehabilitation and Compensation Act. If your claim is accepted by ACC, you still might not get any compensation. This depends on a number of factors such as whether you are an earner or nonearner. ACC usually provides only partial reimbursement of costs and expenses and there may be no lump sum compensation payable. There is no cover for mental injury unless it is a result of physical injury. If you have ACC cover, generally this will affect your right to sue the investigators. If you have any questions about ACC, contact your nearest ACC office or the Principal Investigator. 
Name:

Date of birth:

Gender (circle one): $\quad$ Female $\quad$ Male

Nationality:

Ethnicity:

Have you ever received a mental health diagnosis or diagnoses? (circle one):

Yes $\quad$ No

If yes, what was the diagnosis or diagnoses?

How old were you when received the diagnosis or diagnoses?

Phone number:

Postal Address:

Email Address:

This questionnaire asks about a number of different things that people sometimes do to hurt themselves. Please be sure to read each question carefully and respond honestly. Often, people who do these kinds of things to themselves keep it a secret, for a variety of reasons. However, honest responses to these questions will provide us with greater understanding and knowledge about these behaviours and the best way to help people. Please answer yes to a question only if you did the behaviour intentionally, or on purpose, to hurt yourself. Do not respond yes if you did something accidentally (e.g., you tripped and banged your head on accident). Also, please be assured that your responses are completely confidential. This questionnaire should take you approximately 15 minutes to complete. 
1. Have you ever intentionally (i.e., on purpose) cut your wrist, arms, or other area(s) of your body (without intending to kill yourself)? (circle one):

Yes No

If yes:

- How old were you when you first did this?

- How many times have you done this?

- When was the last time you did this?

- How many years have you been doing this? (If you are no longer doing this, how many years did you do this before you stopped?)

- Has this behaviour ever resulted in hospitalisation or injury severe enough to require medical treatment?

2. Have you ever intentionally (i.e., on purpose) burned yourself with a cigarette (circle one):

Yes No

If yes:

- How old were you when you first did this?

- How many times have you done this?

- When was the last time you did this?

- How many years have you been doing this? (If you are no longer doing this, how many years did you do this before you stopped?)

- Has this behaviour ever resulted in hospitalisation or injury severe enough to require medical treatment?

3. Have you ever intentionally (i.e., on purpose) burned yourself with a lighter or a match? (circle one):

Yes No

If yes:

- How old were you when you first did this?

- How many times have you done this?

- When was the last time you did this?

- How many years have you been doing this? (If you are no longer doing this, how many years did you do this before you stopped?)

- Has this behaviour ever resulted in hospitalisation or injury severe enough to require medical treatment? 
4. Have you ever intentionally (i.e., on purpose) carved words into your skin? (circle one):

Yes

No

If yes:

- How old were you when you first did this?

- How many times have you done this?

- When was the last time you did this?

- How many years have you been doing this? (If you are no longer doing this, how many years did you do this before you stopped?)

- Has this behaviour ever resulted in hospitalisation or injury severe enough to require medical treatment?

5. Have you ever intentionally (i.e., on purpose) carved pictures, designs, or other marks into your skin? (circle one):

Yes

No

If yes:

- How old were you when you first did this?

- How many times have you done this?

- When was the last time you did this?

How many years have you been doing this? (If you are no longer doing this, how many years did you do this before you stopped?)

- Has this behaviour ever resulted in hospitalisation or injury severe enough to require medical treatment?

6. Have you ever intentionally (i.e., on purpose) severely scratched yourself, to the extent that scarring or bleeding occurred? (circle one):

Yes

No

If yes:

- How old were you when you first did this?

- How many times have you done this?

- When was the last time you did this?

- How many years have you been doing this? (If you are no longer doing this, how many years did you do this before you stopped?)

- Has this behaviour ever resulted in hospitalisation or injury severe enough to require medical treatment? 
7. Have you ever intentionally (i.e., on purpose) bitten yourself, to the extent that you broke the skin? (circle one):

Yes

No

If yes:

- How old were you when you first did this?

- How many times have you done this?

- When was the last time you did this?

- How many years have you been doing this? (If you are no longer doing this, how many years did you do this before you stopped?)

- Has this behaviour ever resulted in hospitalisation or injury severe enough to require medical treatment?

8. Have you ever intentionally (i.e., on purpose) rubbed sandpaper on your body? (circle one):

Yes No

\section{If yes:}

- How old were you when you first did this?

- How many times have you done this?

- When was the last time you did this?

- How many years have you been doing this? (If you are no longer doing this, how many years did you do this before you stopped?)

- Has this behaviour ever resulted in hospitalisation or injury severe enough to require medical treatment?

9. Have you ever intentionally (i.e., on purpose) dropped acid onto your skin? (circle one):

Yes No

If yes:

- How old were you when you first did this?

- How many times have you done this?

- When was the last time you did this?

- How many years have you been doing this? (If you are no longer doing this, how many years did you do this before you stopped?)

- Has this behaviour ever resulted in hospitalisation or injury severe enough to require medical treatment? 
10. Have you ever intentionally (i.e., on purpose) used bleach or oven cleaner to scrub your skin? (circle one):

Yes

No

If yes:

- How old were you when you first did this?

- How many times have you done this?

- When was the last time you did this?

- How many years have you been doing this? (If you are no longer doing this, how many years did you do this before you stopped?)

- Has this behaviour ever resulted in hospitalisation or injury severe enough to require medical treatment?

11. Have you ever intentionally (i.e., on purpose) stuck sharp objects such as needles, pins, staples, etc. into your skin, not including tattoos, ear piercing, needles used for drug use, or body piercing? (circle one):

Yes

No

If yes:

- How old were you when you first did this?

- How many times have you done this?

- When was the last time you did this?

- How many years have you been doing this? (If you are no longer doing this, how many years did you do this before you stopped?)

- Has this behaviour ever resulted in hospitalisation or injury severe enough to require medical treatment?

12. Have you ever intentionally (i.e., on purpose) rubbed glass into your skin? (circle one):

Yes No

If yes:

- How old were you when you first did this?

- How many times have you done this?

- When was the last time you did this?

- How many years have you been doing this? (If you are no longer doing this, how many years did you do this before you stopped?)

- Has this behaviour ever resulted in hospitalisation or injury severe enough to require medical treatment? 
13. Have you ever intentionally (i.e., on purpose) broken your own bones? (circle one):

Yes

No

If yes:

- How old were you when you first did this?

- How many times have you done this?

- When was the last time you did this?

- How many years have you been doing this? (If you are no longer doing this, how many years did you do this before you stopped?)

- Has this behaviour ever resulted in hospitalisation or injury severe enough to require medical treatment?

14. Have you ever intentionally (i.e., on purpose) banged your head against something, to the extent that you caused a bruise to appear? (circle one):

Yes

No

If yes:

- How old were you when you first did this?

- How many times have you done this?

- When was the last time you did this?

- How many years have you been doing this? (If you are no longer doing this, how many years did you do this before you stopped?)

- Has this behaviour ever resulted in hospitalisation or injury severe enough to require medical treatment?

15. Have you ever intentionally (i.e., on purpose) punched yourself, to the extent that you caused a bruise to appear? (circle one):

Yes

No

If yes:

- How old were you when you first did this?

- How many times have you done this?

- When was the last time you did this?

- How many years have you been doing this? (If you are no longer doing this, how many years did you do this before you stopped?)

- Has this behaviour ever resulted in hospitalisation or injury severe enough to require medical treatment? 
16. Have you ever intentionally (i.e., on purpose) prevented wounds from healing? (circle one):

Yes No

If yes:

- How old were you when you first did this?

- How many times have you done this?

- When was the last time you did this?

- How many years have you been doing this? (If you are no longer doing this, how many years did you do this before you stopped?)

- Has this behaviour ever resulted in hospitalisation or injury severe enough to require medical treatment?

17. Have you ever intentionally (i.e., on purpose) done anything else to hurt yourself that was not asked about in this questionnaire? If yes, what did you do to hurt yourself?

- How old were you when you first did this?

- How many times have you done this?

- When was the last time you did this?

- How many years have you been doing this? (If you are no longer doing this, how many years did you do this before you stopped?)

- Has this behaviour ever resulted in hospitalisation or injury severe enough to require medical treatment?

Thank you for completing this questionnaire.

Your time and contribution to this research is much appreciated. 
Deliberate self-harm can be a very difficult topic to talk about and as a result, some of the questions you have been asked about today may have brought up past memories or feelings that are unpleasant or distressing. If this is the case, please feel free to contact me on (04) 4635233 extension 8605 or at robyn.langlands@vuw.ac.nz. Alternatively, you may wish to call, email or check out the website of one of the following support organisations:

\begin{tabular}{|c|c|}
\hline LifeLine & Youthline \\
\hline $\begin{array}{l}\text { Free telephone counselling } \\
\text { Available } 24 \text { hours a day, 7days a week } \\
\text { Ph: } 0800111777 \\
\text { Website: http://www.lifeline.org.nz/ }\end{array}$ & $\begin{array}{l}\text { Free telephone counselling } \\
\text { Available } 24 \text { hours a day, 7days a week } \\
\text { Ph: } 0800376633 \\
\text { Website: }\end{array}$ \\
\hline $\begin{array}{l}\text { Website: http://www.lifeline.org.nz/ } \\
\text { Free email counselling } \\
\text { Email: chris@lifeline.co.nz } \\
\text { Website: http://www.elifeline.co.nz/ }\end{array}$ & $\begin{array}{l}\text { Website: http://www.youthline.co.nz/ } \\
\text { Email or Text Support } \\
\text { Email: talk@youthline.co.nz } \\
\text { TXT: } 0274 \text { YOUTHS (027 } 4968 \text { 847) }\end{array}$ \\
\hline $\begin{array}{l}\text { Mensline } \\
\text { Free telephone Counselling } \\
\text { Available 5:30pm-11pm, } 7 \text { nights a week } \\
\text { Ph: 0800 MENSLINE (636 754) } \\
\text { Website: http://www.mensline.org.nz/ }\end{array}$ & $\begin{array}{l}\text { Alcohol and Drug Helpline } \\
\text { Available 10am-10pm daily } \\
\text { Ph: } 0800787797 \\
\text { Website: } \\
\text { http://www.adanz.org.nz/ADANZ/Home }\end{array}$ \\
\hline $\begin{array}{l}\text { Warmline } \\
\text { Free phone support service staffed by } \\
\text { volunteers specifically for people who use } \\
\text { mental health services. Volunteers are people } \\
\text { who have used mental health services } \\
\text { themselves. } \\
\text { Available } 7.00 \mathrm{pm}-1.00 \mathrm{am} \text {. Tuesday-Sunday } \\
\text { Ph: } 0800200207\end{array}$ & $\begin{array}{l}\text { Buddies } \\
\text { Buddies offers peer support to people who } \\
\text { self identify as experiencing a mental illness } \\
\text { or who have used mental health services. } \\
\text { Ph: (04) } 3852104 \text { or (021) } 960060 \\
\text { Email: buddies.wn@paradise.net.nz } \\
\text { Visit: Level } 6 \text { NZEI House } 178 \text { Willis } \\
\quad \text { Street, Wellington }\end{array}$ \\
\hline
\end{tabular}


VICTORIA UNIVERSITY OF WELLINGTON

Te Whare Wananga o te Upoko o te Ika a Maui

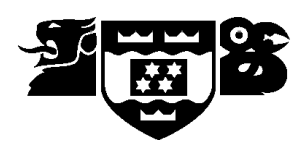

\section{Interview Questions}

Please read the following questions carefully. The purpose of this study is to understand how you experience self-harm from your perspective, while at the same time addressing the research questions listed below. As a result, Robyn cannot guarantee that she will ask you all of these questions or that these will be the only questions she will ask you during the interview(s). The questions do, however, provide you with an idea about what kinds of topics will be discussed during the interview. Please consider carefully if you would be comfortable answering these questions before you decide whether you would like to participate in an interview.

- Can you tell me about the last time that you deliberately harmed yourself without intending to kill yourself?

- Can you tell me as much as you can remember about what led up to this episode of self-harm?

- Can you describe for me how you were feeling prior to harming yourself?

- How did you feel during this particular episode of self-harm?

- How did you feel afterwards?

- Can you tell me what you were thinking about prior to harming yourself?

- What were you thinking about during this particular episode of self-harm?

- Did those thoughts change in any way after the episode of self-harm? [If yes...] What were you thinking about after you had harmed yourself?

- Did you have to deal with any particular consequences from that episode of self-harm? [If yes...] Can you tell me about them?

- Thinking about what we've talked about in relation to this particular episode of self-harm, would you consider this a typical episode of self-harm for you? [If no...] What was different about this episode compared to other times in the past when you've harmed yourself?

- Is there anything that you'd like to tell me about in relation to your self-harm that we haven't discussed? 


\section{VICTORIA UNIVERSITY OF WELLINGTON \\ Te Whare Wananga o te Upoko o te Ika a Maui}

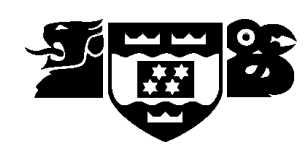

\section{Consent to Contact Clinician}

I give permission for the Principal Investigator to inform my mental health clinician that I am participating in this study.

I give permission for the Principal Investigator to confirm with my clinician any mental health diagnoses that I have if this is necessary.

Full name:

Signature

Date: 


\section{Exploring the experiences of adolescents and young adults who engage in non-suicidal deliberate self-harm behaviours}

\section{Consent Form}

- I have read and I understand the information sheet dated June 2008 for volunteers taking part in the study designed to explore the deliberate self-harm experiences of adolescents and young adults.

- I have read the list of interview questions for the study.

- I have had the opportunity to discuss this study. I am satisfied with the answers I have been given.

- I have had the opportunity to use whanau support or a friend to help me ask questions and understand the study.

- I understand that taking part in this study is voluntary (my choice) and that I may withdraw from the study at any time and this will in no way affect my continuing health care.

- I understand that my participation in this study is confidential and that no material which could identify me will be used in any reports on this study.

- I understand that my interview will be audio-taped.

- I understand the compensation provisions for this study.

- I have had time to consider whether to take part.

- I know who to contact if the study distresses me in any way.

- I know who to contact if I have any questions about the study. 


\section{Please complete the following:}

I would like to receive a copy of my interview transcript.

$\square$ Yes $\square$ No

I would like to receive a summary of the research results.

$\square$ Yes $\square$ No

I would like to be contacted by Robyn Langlands in the future with regards to

$\square$ Yes $\square$ No other studies she is conducting on deliberate self-harm behaviours for her PhD.

Please write your contact details below.

What is the best time of day to contact you?

(please circle one):
Morning
Afternoon
Evening

Is the form of contact you've chosen (please circle one):

Private

Shared

(e.g. personal

(e.g. your

email address

family's

or cell

home phone

number)

number)

I (full name) hereby consent to take part in this study.

Date:

Signature:

Full names of Principal Investigator:

Contact Phone Number for Principal Investigator:

Project explained by:

Project role:

Date:

Signature: 
DATE:

This questionnaire should take approximately 15 minutes to complete. Please note that when a question refers to 'the study', this includes both the first questionnaire you completed and the interview(s) you participated in. Please circle the response that best describes your experiences of taking part in this study:

1) The study was explained thoroughly to me before I took part.

1

Strongly disagree

\section{2}

Disagree

\section{3}

Not sure

\section{4}

Agree
5

Strongly agree

2) It was my choice to take part in the study (i.e., I could have said no even if other people wanted me to say yes).

1

Strongly disagree
2

Disagree
3

Not sure
4

Agree
5

Strongly agree

3) I knew I could skip questions or parts of the study if I wanted to.

1

Strongly disagree
2

Disagree
3

Not sure
4

Agree
5

Strongly agree

4) I knew I could stop being in the study at any time.

1

Strongly disagree
2

Disagree
3

Not sure
4

Agree
5

Strongly agree

5) During the interview, I knew that I could ask to take a break whenever I wanted.

1

Strongly disagree
2

Disagree
3

Not sure
4

Agree
5

Strongly agree 
6) The interviewer made me feel comfortable during the interview.

1

Strongly disagree
2

Disagree
3

Not sure
4

Agree
5

Strongly agree

7) The interviewer showed respect for my feelings and experiences during the interview.

1

Strongly disagree
2

Disagree
3

Not sure
4

Agree
5

Strongly agree

8) I am glad that I took part in this study.

1

Strongly disagree
2

Disagree
3

Not sure
4

Agree
5

Strongly agree

9) Being in this study made me feel distressed.

1

Strongly disagree
2

Disagree
3

Not sure
4

Agree
5

Strongly agree

10) Being in this study made me feel upset.

1

Strongly disagree
2

Disagree
3

Not sure
4

Agree
5

Strongly agree

11) Being in this study made me feel good about myself.

1

Strongly disagree
2

Disagree
3

Not sure
4

Agree
5

Strongly agree

12) I regret participating in this study.

1

Strongly disagree
2

Disagree
3

Not sure
4

Agree
5

Strongly agree

13) Being in this study made me feel sad.

1

Strongly disagree
2

Disagree
3

Not sure
4

Agree
5

Strongly agree

14) I believe that by being in the study I am helping other people who self-harm.

1

Strongly disagree
2

Disagree
3

Not sure
4

Agree
5

Strongly agree 
15) Knowing what I know now about participating in the study, I would still choose to take part in this research.

1

Strongly disagree
2

Disagree
3

Not sure
4

Agree
5

Strongly agree

16) Do you have any suggestions as to how to improve research studies about deliberate self-harm? If yes, please write them below.

17) Do you have any other comments about your experiences of participating in this study? If yes, please write them below. 
Thank you very much for completing this questionnaire. Your feedback and suggestions will be used to improve future studies about deliberate self-harm. 


\section{Exploring the functions of non-suicidal self-injury: Information sheet}

\section{Robyn Langlands}

PhD Student, School of Psychology

Victoria University of Wellington

Email: robyn.langlands@vuw.ac.nz

\section{Dr Marc Wilson}

Supervisor, School of Psychology

Victoria University of Wellington

Email: marc.wilson@vuw.ac.nz

Phone: (04) 4635225

\section{What is the purpose of this research?}

The purpose of this research is to explore the thoughts, feelings, and events that lead people to intentionally injure themselves. It is important to understand why people hurt themselves on purpose in order to develop the most appropriate treatment and prevention programmes.

\section{Who is conducting the research?}

Robyn Langlands is conducting the study as part of her $\mathrm{PhD}$ thesis. Dr Marc Wilson is supervising the research. This research has been approved by the University ethics committee.

Who is being asked to participate in this study?

You can participate in this study, if you...

- are 16 years of age or older,

- live in Aotearoa/New Zealand.

- have injured yourself on purpose, without intending to kill yourself, one or more times in the past 12 months, and

- were not experiencing psychosis (e.g., delusions or hallucinations) or mania when you most recently injured yourself on purpose.

What is involved if you agree to participate?

If you agree to participate in this study, you will complete a survey about non-suicidal self-injury (also called deliberate self-harm). This survey contains lots of questions about non-suicidal self-injury. For example, you will be asked to think back to the time when you most recently injured yourself on purpose, without intending to kill yourself, and to answer questions about how you felt before and after that episode. We expect that the survey will take you no more than 45 minutes to complete. You are free to withdraw from this study at any point before your survey has been completed. You also will be given the option of receiving a summary of the research results.

Privacy and Confidentiality

- We will keep your data for at least five years after publication.

- You will never be identified in this research project or in any other presentation or publication. The information you provide will be coded by number only.

- In accordance with the requirements of some scientific journals and organisations, your coded data may be shared with other competent researchers.

- Your coded data may be used in other, related studies.

- A copy of the coded data will remain in the custody of Robyn Langlands and Dr Wilson.

What happens to the information you provide?

The data you provide may be used for one or more of the following purposes:

- The overall findings may be submitted for publication in a scientific journal, or presented at scientific conferences.

- The overall findings may form part of a PhD thesis that will be submitted for assessment.

\section{Are there any risks?}

Non-suicidal self-injury can be a very difficult topic to answer questions about and there is a risk that some of the questions asked may bring up past memories or feelings that are unpleasant or distressing. If appropriate, you may wish to ask a support person to be available while you take the survey. If you do become distressed while completing the survey, you can stop filling it in at any time. Also, there will be a list of options for further support at the end of the survey. 


\section{Exploring the functions of non-suicidal self-injury: Information sheet}

What are the benefits?

There is no guarantee that you will receive any benefits from participating in this research. However, this study does have the potential to lead to better mental health care and support for people who engage in non-suicidal self-injury.

Will $I$ be paid to participate in this project?

You will be given the option of entering into a draw to win one of two iPod Shuffles (2GB) for completing the survey. 


\section{Exploring the functions of non-suicidal self-injury: Information sheet}

To take part in this study you must:

- be 16 years of age or older

- live in Aotearoa/New Zealand

- have injured yourself on purpose, WITHOUT intending to kill yourself, one or more times in the past 12 months

- NOT have experienced psychosis (e.g., delusions or hallucinations) or mania during your most recent episode of non-suicidal self-injury

\section{Are ALL FOUR of these statements true for you?}

$\bigcirc$ Yes

No 


\section{Exploring the functions of non-suicidal self-injury: Information sheet}

Thank you for your time. Unfortunately, you do not meet the criteria to participate in this survey. Please close your browser (e.g., Internet Explorer, Firefox) to exit this survey. 


\section{Exploring the functions of non-suicidal self-injury: Information sheet}

Thank you for your time. Unfortunately, you do not meet the criteria to participate in this survey. Please close your browser (e.g., Internet Explorer, Firefox) to exit this survey. 


\section{Exploring the functions of non-suicidal self-injury: Information sheet}

You meet the criteria to participate in this survey. If you would like to take part, please make sure you have 30-45 minutes of time available before starting the survey. If you close the survey before completing it you will not be able to log back in to carry on from where you left off.

Before you begin the survey, please type your email address in the space provided. Everyone who takes part in this study will be emailed a copy of the debriefing information which includes contact details for support organisations. Your email address will not be linked to your survey answers. Your survey answers will be anonymous.

Email Address: 


\section{Exploring the functions of non-suicidal self-injury}

\section{Section One}

In order to understand more about your responses to later parts of the survey, we would like to know a bit more about your background.

\section{Are youn=.}

Female

Male

Other (Please specify)

\section{How old are you?}

\section{Which ethnic group do you belong to? (Please select all that apply)}
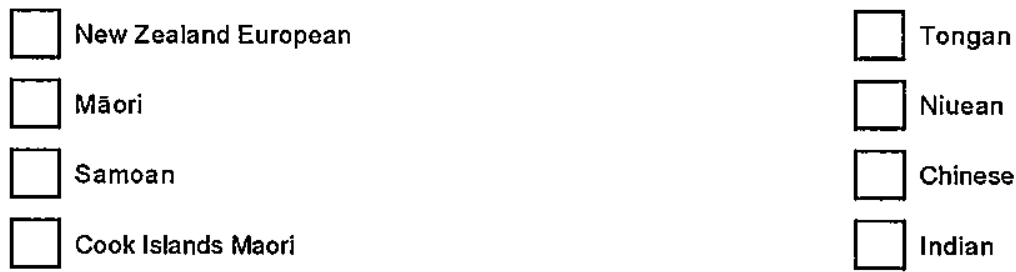

Other (Please state: e.g., Dutch, Japanese, Tokelauan)

\section{What region do you live in?}
Northland
Taranaki
West Coast
C Auckland
Manawatu-Wanganui
Canterbury
Caikato
$\bigcirc$ Wellington
Otago
( Bay of Plenty
(Tasman
Southland
East Cape
CNelson
O Hawke's Bay
(Marlborough
Other (Please specify)

\section{Please choose the description that best fits how you think about yourself:}

$100 \%$ heterosexual

Mostly heterosexual, but somewhat attracted to people of your own sex

Bisexual-that is, attracted to men and women equally

Mostly homosexual (gay/lesbian), but somewhat attracted to people of the opposite sex

(100\% homosexual (gay/lesbian)

Not sexually attracted to either males or females 


\section{Exploring the functions of non-suicidal self-injury}

\section{6 . What is your religion?}
No religion
Hindu
Christian
Buddhist
Muslim Jewish
Other (Please specify)

\section{Please select ONE answer that BEST describes your current occupation:}
Secondary school student
Full-time employment
Tertiary Student (e.g., university, polytechnic)
Unemployed
Part-time employment
Stay-at-home parent
Other (Please specify)

\section{What is your highest level of education? (Choose one only)}

No qualification

High school qualification

Trade or technical qualification (e.g., plumber, builder)

Degree or diploma

Postgraduate qualification

Other (Please specify)

9. Have you ever received a mental health diagnosis?
$\bigcirc$ Yes
No

10. If you answered YES, please select all that apply:
Depression Obsessive-Compulsive Disorder
Bipolar Disorder Post-Traumatic Stress Disorder
Anorexia Anxiety Disorder
$\square$ Bulimia Substance Use Disorder
Borderline Personality Disorder Schizophrenia

Other (Please specify) 


\section{Exploring the functions of non-suicidal self-injury}

11. Please choose the statement or phrase that best applies to you. Have you ever thought about or attempted to kill yourself? (Choose one only)
Never
It was just a brief passing thought
I have had a plan at least once to kill myself but did not try to do it
I have had a plan at least once to kill myself and really wanted to die
I have attempted to kill myself, but did not want to die
I have attempted to kill myself, and really hoped to die 


\section{Section Two}

The following section asks about a number of different things that people sometimes do to hurt themselves. Please be sure to read each question carefully and respond honestly. Often, people who do these kinds of things to themselves keep it a secret, for a variety of reasons. However, honest responses to these questions will provide us with greater understanding and knowledge about these behaviours and the best way to help people. Please answer yes to a question only if you did the behaviour intentionally, or on purpose, to hurt yourself. Do not respond yes if you did something accidentally (e.g., you tripped and banged your head on accident). Also, please be assured that your responses are completely confidential.

1. Have you ever intentionally (i.e., on purpose) cut your wrist, arms, or other area(s) of your body (without intending to kill yourself)? (Select one):

$\bigcirc$ Yes

No

If YES, how many times have you done this? (If you can't remember, please estimate the number of times (e.g., $5,10,100)$ you have done this)

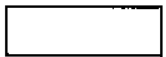

2. Have you ever intentionally (i.e., on purpose) burned yourself with a cigarette, lighter, or a match? (Select one):

$\bigcirc$ Yes

$\bigcirc$ No

If YES, how many times have you done this? (If you can't remember, please estimate the number of times (e.g., 5, 10, 100) you have done this)

3. Have you ever intentionally (i.e., on purpose) carved words, pictures, designs, or other marks into your skin? (Select one):

Ones
No

If YES, how many times have you done this? (If you can't remember, please estimate the number of times (e.g., 5, 10, 100) you have done this)

4. Have you ever intentionally (i.e., on purpose) severely scratched yourself, to the extent that scarring or bleeding occurred? (Select one):

Y Yes

No

if YES, how many times have you done this? (If you can't remember, please estimate the number of times (e.g., 5, 10, 100) you have done this) 


\section{Exploring the functions of non-suicidal self-injury}

5. Have you ever intentionally (i.e., on purpose) bitten yourself, to the extent that you broke the skin? (Select one):

$\bigcirc$ Yes

No

If YES, how many times have you done this? (If you can't remember, please estimate the number of times (e.g., 5, 10, 100) you have done this)

6. Have you ever intentionally (i.e., on purpose) rubbed sandpaper on your body? (Select one):
Y Yes
No

If YES, how many times have you done this? (If you can't remember, please estimate the number of times (e.g., 5, 10, 100) you have done this)

7. Have you ever intentionally (i.e., on purpose) dripped acid onto your skin? (Select one):

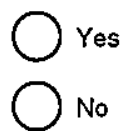

If YES, how many times have you done this? (If you can't remember, please estimate the number of times (e.g., 5, 10, 100) you have done this)

8. Have you ever intentionally (i.e., on purpose) used bleach or oven cleaner to scrub your skin? (Select one):

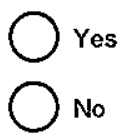

If YES, how many times have you done this? (If you can't remember, please estimate the number of times (e.g., 5, 10, 100) you have done this)

9. Have you ever intentionally (i.e., on purpose) stuck sharp objects such as needles, pins, staples, etc. into your skin, not including tattoos, ear piercing, needles used for drug use, or body piercing? (Select one):

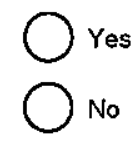

If YES, how many times have you done this? (If you can't remember, please estimate the number of times (e.g.. 5, 10, 100) you have done this) 


\section{Exploring the functions of non-suicidal self-injury}

10. Have you ever intentionally (i.e., on purpose) rubbed glass into your skin? (Select one):

$\bigcirc$ Yes

No

If YES, how many times have you done this? (If you can't remember, please estimate the number of times (e.g., 5, 10, 100) you have done this)

11. Have you ever intentionally (i.e., on purpose) broken your own bones? (Select one):

Yes

$\bigcirc$ No

If YES, how many times have you done this? (If you can't remember, please estimate the number of times (e.g., 5, 10, 100) you have done this)

12. Have you ever intentionally (i.e., on purpose) banged your head against something, to the extent that you caused a bruise to appear? (Select one):

Y Yes

No

If YES, how many times have you done this? (If you can't remember, please estimate the number of times (e.g., 5, 10, 100) you have done this)

13. Have you ever intentionally (i,ex, on purpose) punched yourself, to the extent that you caused a bruise to appear? (Select one):

Yes

No

If YES, how many times have you done this? (If you can't remember, please estimate the number of times (e.g., 5, 10, 100) you have done this)

14. Have you ever intentionally (i.e., on purpose) prevented wounds from healing? (Select one):

$\bigcirc$ Yes

No

If YES, how many times have you done this? (If you can't remember, please estimate the number of times (e.g., 5, 10, 100) you have done this) 


\section{Exploring the functions of non-suicidal self-injury}

15. Have you ever intentionally (i.e., on purpose) done anything else to hurt yourself that was not asked about in this questionnaire? (Select one):

$\bigcirc$ Yes

No

If YES, what did you do to hurt yourself? How many times have you done this? (If you can't remember, please estimate the number of times (e.g., 5, 10, 100) you have done this)

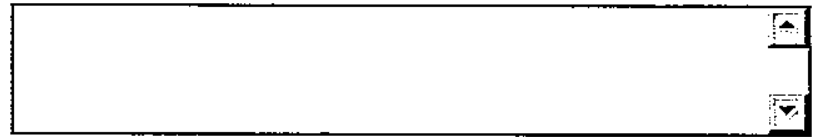

16. How old were you when you first hurt yourself on purpose? (If you can't remember, please estimate how old you were) 


\section{Exploring the functions of non-suicidal self-injury}

\section{Section Three}

This inventory was written to help us better understand the experience of non-suicidal self-injury. Below is a list of statements that may or may not be relevant to your experience of self-injury. Please select the option that indicates how relevant each statement is for you:

\section{1. "When I self-injure, I am ...}

... calming myself down

... creating a boundary between myself and others

... punishing myself

... giving myself a way to care for myself (by attending to the wound)

... causing pain so I will stop feeling numb

... avoiding the impulse to attempt suicide

... doing something to generate excitement or exhilaration

... bonding with peers

... letting others know the extent of my emotional pain

... seeing if I can stand the pain

... creating a physical sign that I feel awful

... getting back at someone

... ensuring that $I$ am self-sufficient

... releasing emotional pressure that has built up inside of me

... demonstrating that I am separate from other people

... expressing anger towards myself for being worthless or stupid

... creating a physical injury that is easier to care for than my emotional distress

... trying to feel something (as opposed to nothing) even if it is physical pain

... responding to suicidal thoughts without actually attempting suicide

... entertaining myself or others by doing something extreme

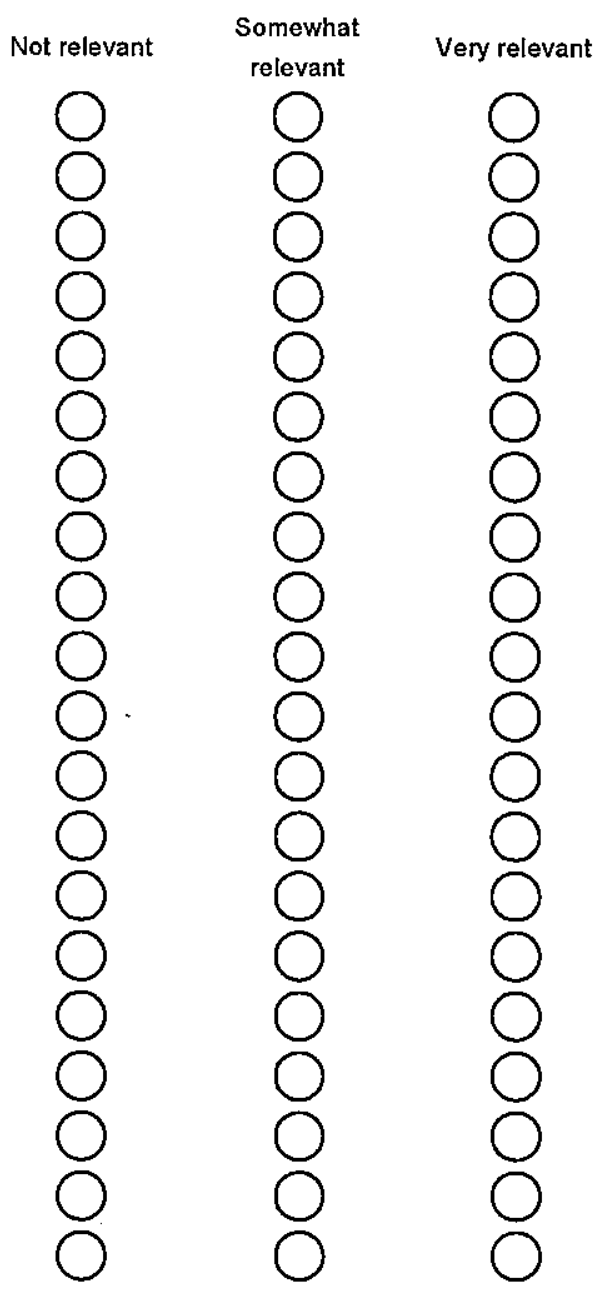




\section{Exploring the functions of non-suicidal self-injury}

\section{Continued...}

"When I self-injure, I am...

... fitting in with others

... seeking care or help from others

... demonstrating I am tough or strong

... proving to myself that my emotional pain is real

... getting revenge against others

... demonstrating that I do not need to rely on others for help

... reducing anxiety, frustration, anger, or other overwhelming emotions

... establishing a barrier between myself and others

... reacting to feeling unhappy with myself or disgusted with myself

... allowing myself to focus on treating the injury, which can be gratifying or satisfying

... making sure I am still alive when I don't feel real

... putting a stop to suicidal thoughts

... pushing my limits in a manner akin to skydiving or other extreme activities

... creating a sign of friendship or kinship with friends or loved ones

... keeping a loved one from leaving or abandoning me

... proving I can take the physical pain

... signifying the emotional distress I'm experiencing

... trying to hurt someone close to me

... establishing that I am autonomous/independent

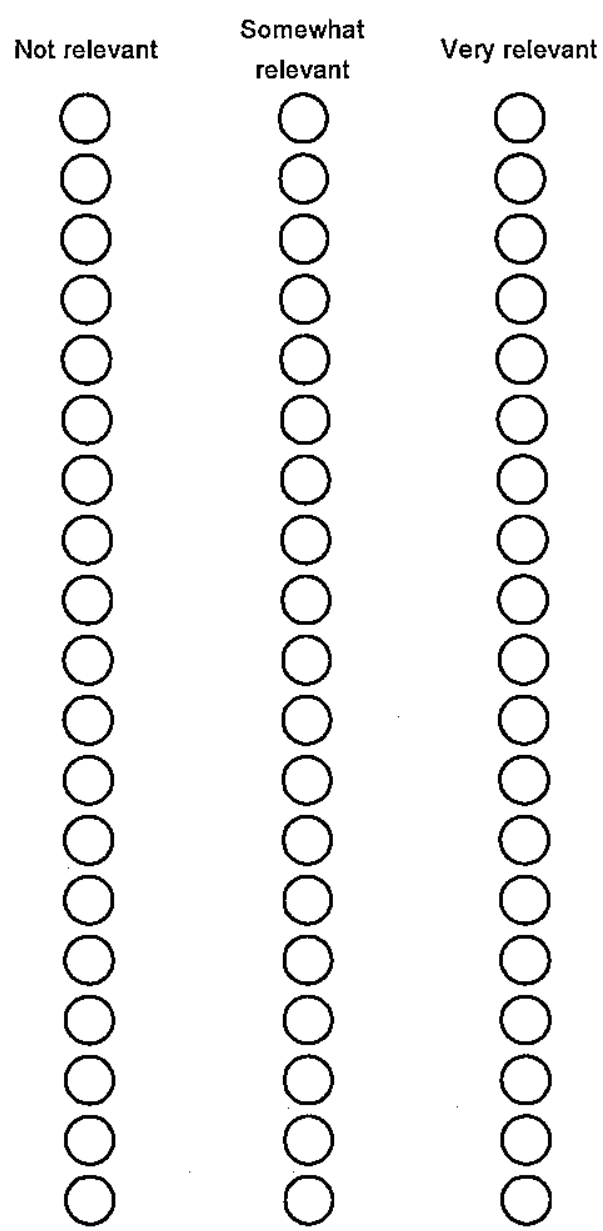




\section{Exploring the functions of non-suicidal self-injury}

\section{Section Four}

Please think back to your MOST RECENT episode of non-suicidal self-injury (i.e., the last time you hurt yourself on purpose without intending to kill yourself) and answer the following questions IN RELATION to that episode.

\section{When did you injure yourself (approximate date - day/month/year)?}

\section{How did you injure yourself? (Please select all that apply)}

$\square$ Cut yourself

Burned yourself

Carved words, pictures, designs, or other marks into your skin

Severely scratched yourself to the extent that scarring or bleeding occurred

Bit yourself to the extent that you broke the skin

Rubbed sandpaper on your body

Dripped acid onto your skin

$\square$ Used bleach or oven cleaner to scrub your skin

Stuck sharp objects (e.g., needles, pins, staples) into your skin (not including tattoos, ear piercing, needles used for drug use, or body piercing)

Rubbed glass into your skin

Broke your own bones

Banged your head against something to the extent that you caused a bruise to appear

Punched yourself to the extent that you caused a bruise to appear

Prevented wounds from healing

Other (Please specify)

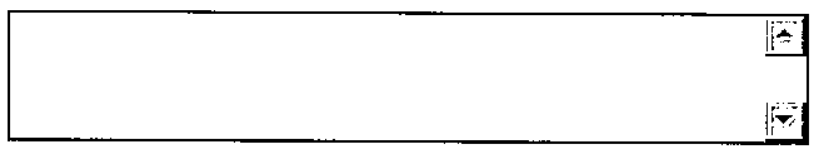

3. Was the injury severe enough to require medical treatment?

$\bigcirc$ Yes

ONo

\section{Did you tell anyone that you had injured yourself on purpose?}
$\bigcirc$ Yes
$\bigcirc$ No 


\section{Exploring the functions of non-suicidal self-injury}

5. If you answered YES, who did you tell? (Please select all that apply)

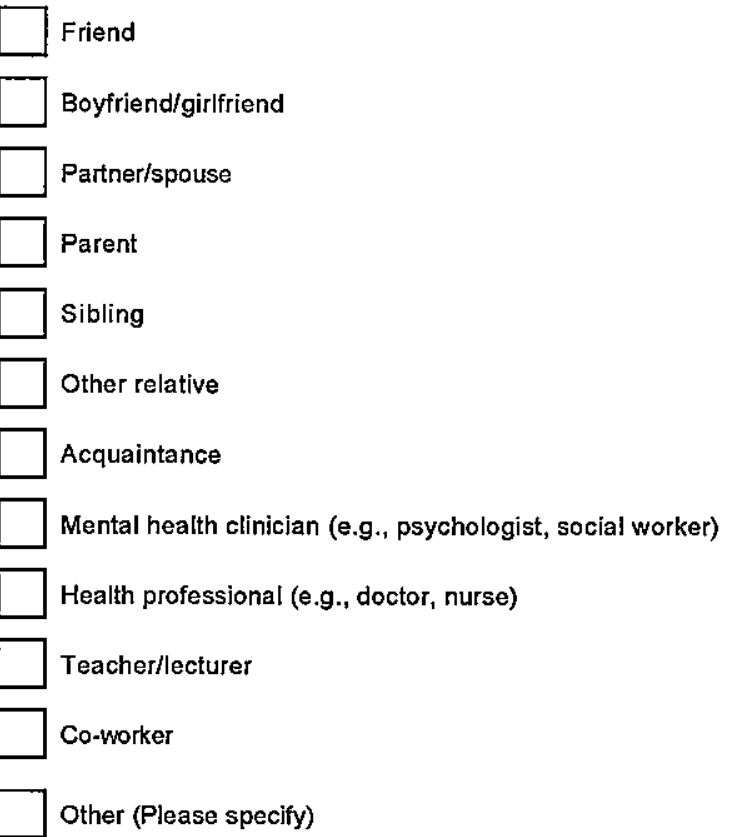

6. Did you drink more than 2 standard drinks of alcohol (i.e., 2 cans of beer $(330 \mathrm{ml}$ each) or $200 \mathrm{ml}$ of table wine or $60 \mathrm{ml}$ of straight spirits) before you injured yourself?
$\bigcirc$ Yes
No
Don't know

7. Did you take any legal drugs/medication (e.g, paracetamol), in excess of the recommended dosage, before you injured yourself?
$\bigcirc$ Yes
$\bigcirc$ No
Don't know

If you answered YES, what drugs/medication did you take?

\section{Did you use any illegal drugs before you injured yourself?}
$\bigcirc$ Yes
$\bigcirc$ No
Don't know

If you answered YES, what drugs did you use? 


\section{Exploring the functions of non-suicidal self-injury}

9. Did something specific happen that led to this most recent episode of self-injury?

$\bigcirc$ Yes

No

10. If you answered YES, please describe briefly what happened and when it happened.

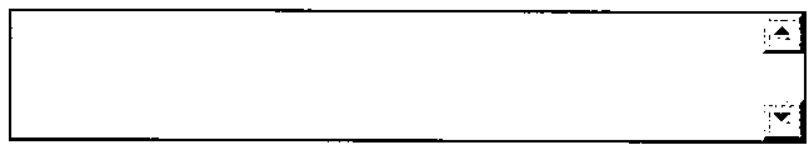

11. How did what happened make you feel?

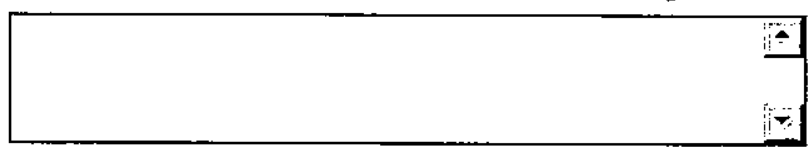




\section{Exploring the functions of non-suicidal self-injury}

\section{Section Five}

In this section, you will be asked to answer the following questions TWICE-once in relation to feelings you may or may not have had BEFORE you injured yourself most recently and once in relation to feelings you may or may not have had AFTER you injured yourself most recently.

\section{This scale consists of a number of words and phrases that} describe different feelings and emotions. Read each item and then mark the appropriate answer in the space next to that word. Indicate to what extent you felt this way BEFORE your MOST RECENT episode of non-suicidal self-injury (i.e., the last time you hurt yourself on purpose without intending to kill yourself). Use the following scale to record your answers:

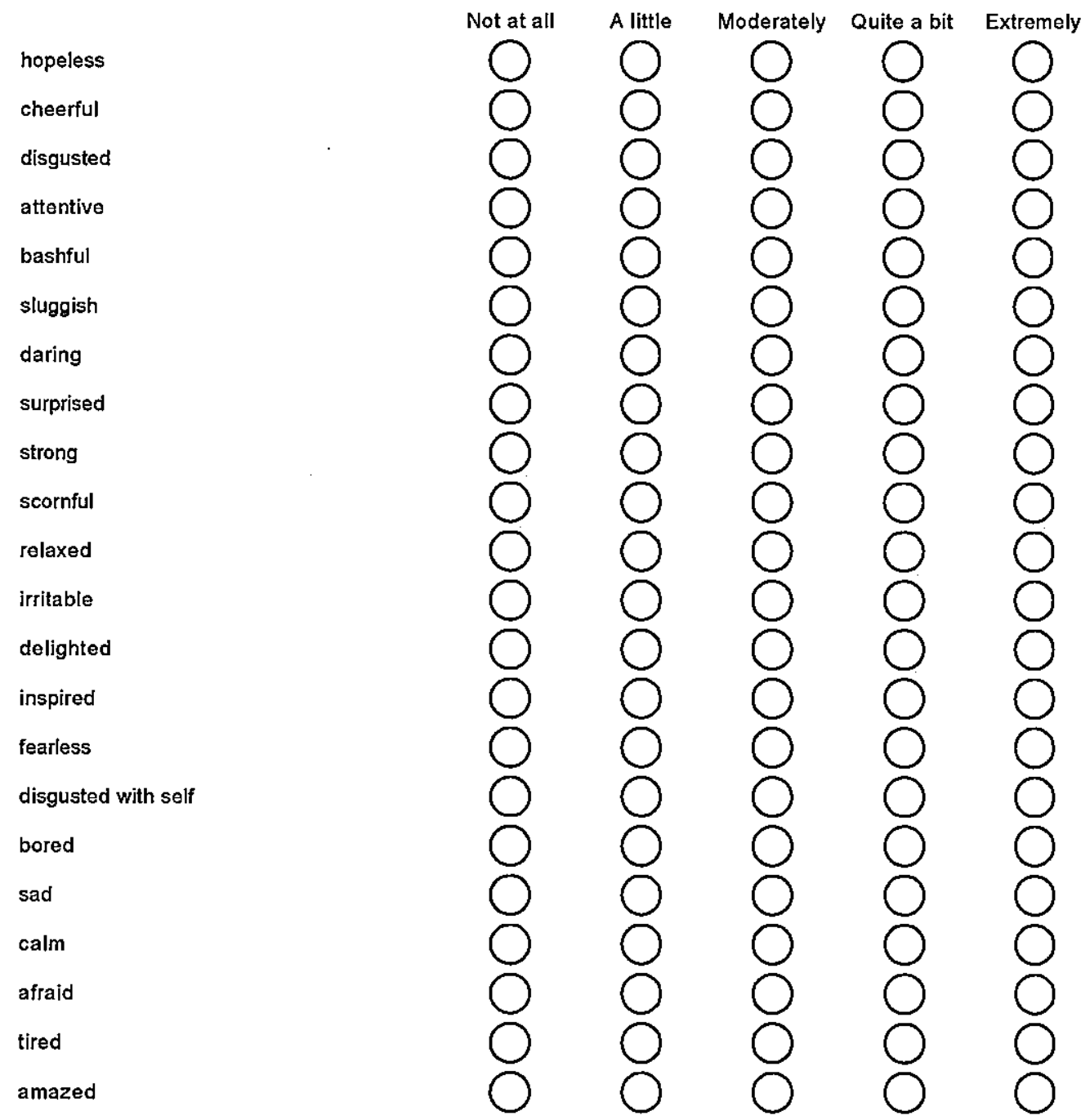




\section{Exploring the functions of non-suicidal self-injury}

\section{Continued...}

shaky

happy

timid

alone

alert

upset

angry

bold

blue

shy

disappointed with self

frustrated

active

guilty

joyful

nervous

Jonely

sleepy

excited

hostile

proud

jittery

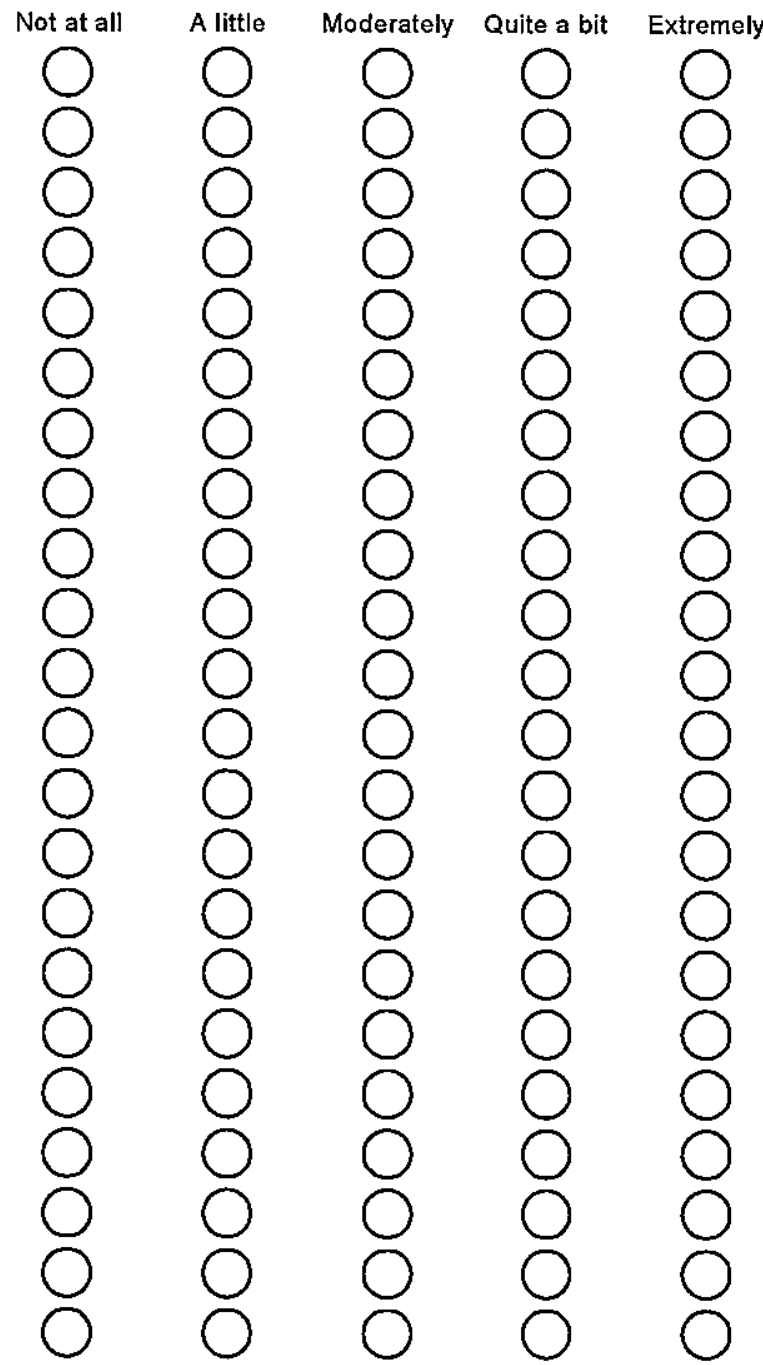




\section{Exploring the functions of non-suicidal self-injury}

\section{Continued...}

lively
ashamed
at ease
enthusiastic
sownhearted
sheepish
distressed
blameworthy
determined
frightened
astonished
interested
loathing
confident
energetic
concentrating
dissatisfied with self




\section{Exploring the functions of non-suicidal self-injury}

1. Read each item and then mark the appropriate answer in the space next to that word.

Indicate to what extent you felt this way AFTER your MOST RECENT episode of non-suicidal self-injury (i.e., the last time you hurt yourself on purpose without intending to kill yourself). Use the following scale to record your answers:

hopeless
cheerful
disgusted
attentive
bashful
surprised
stronging
scornful
relaxed
irritable
delighted
inspired
fearless
disgusted with self
bored
sad
calm
afraid
tired
amazed




\section{Exploring the functions of non-suicidal self-injury}

\section{Continued...}

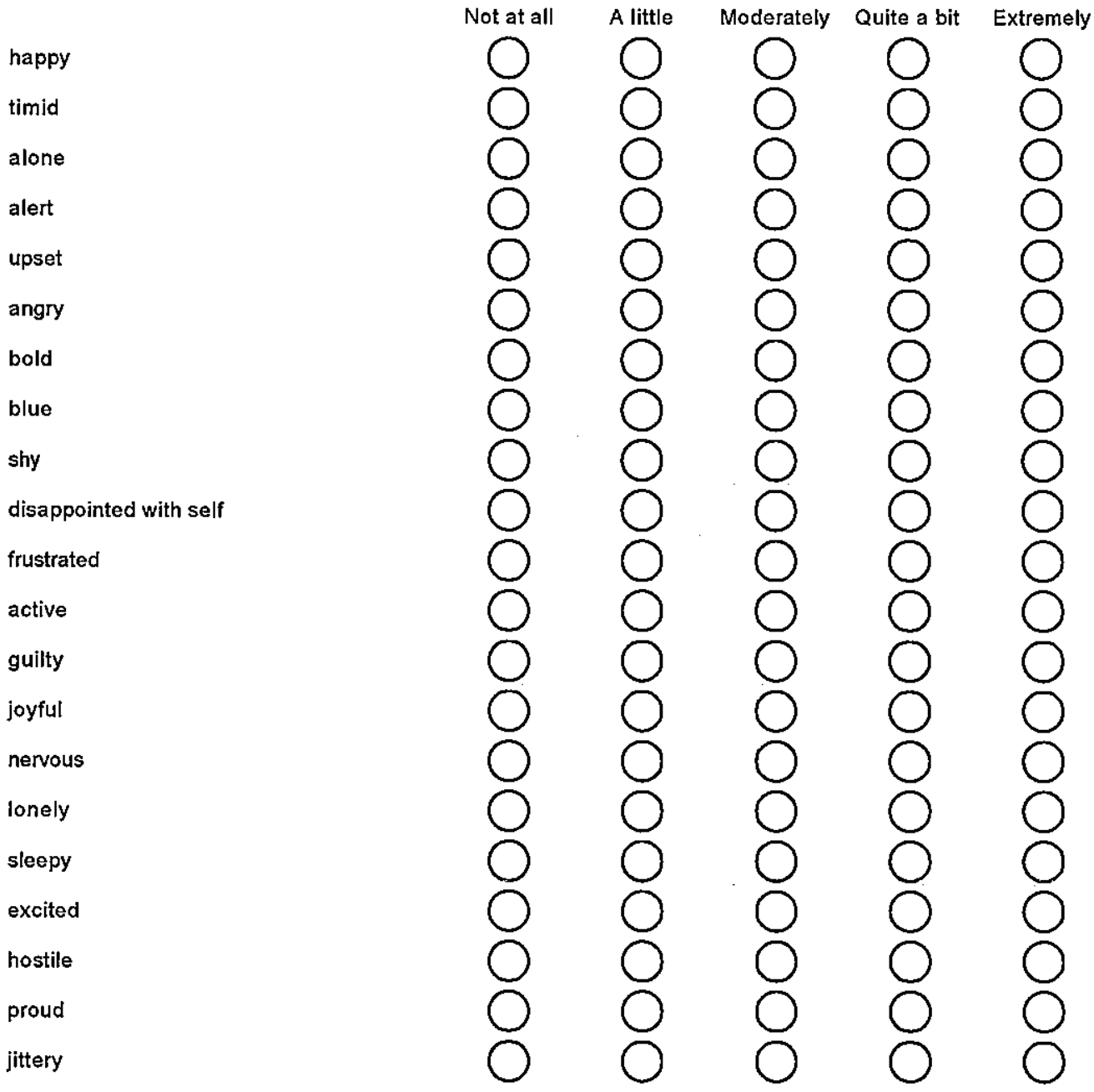




\section{Exploring the functions of non-suicidal self-injury}

\section{Continued...}

lively

ashamed

at ease

scared

drowsy

hopeful

empty inside

angry at self

enthusiastic

downhearted

sheepish

distressed

blameworthy

determined

frightened

astonished

interested

loathing

confident

energetic

concentrating

dissatisfied with self

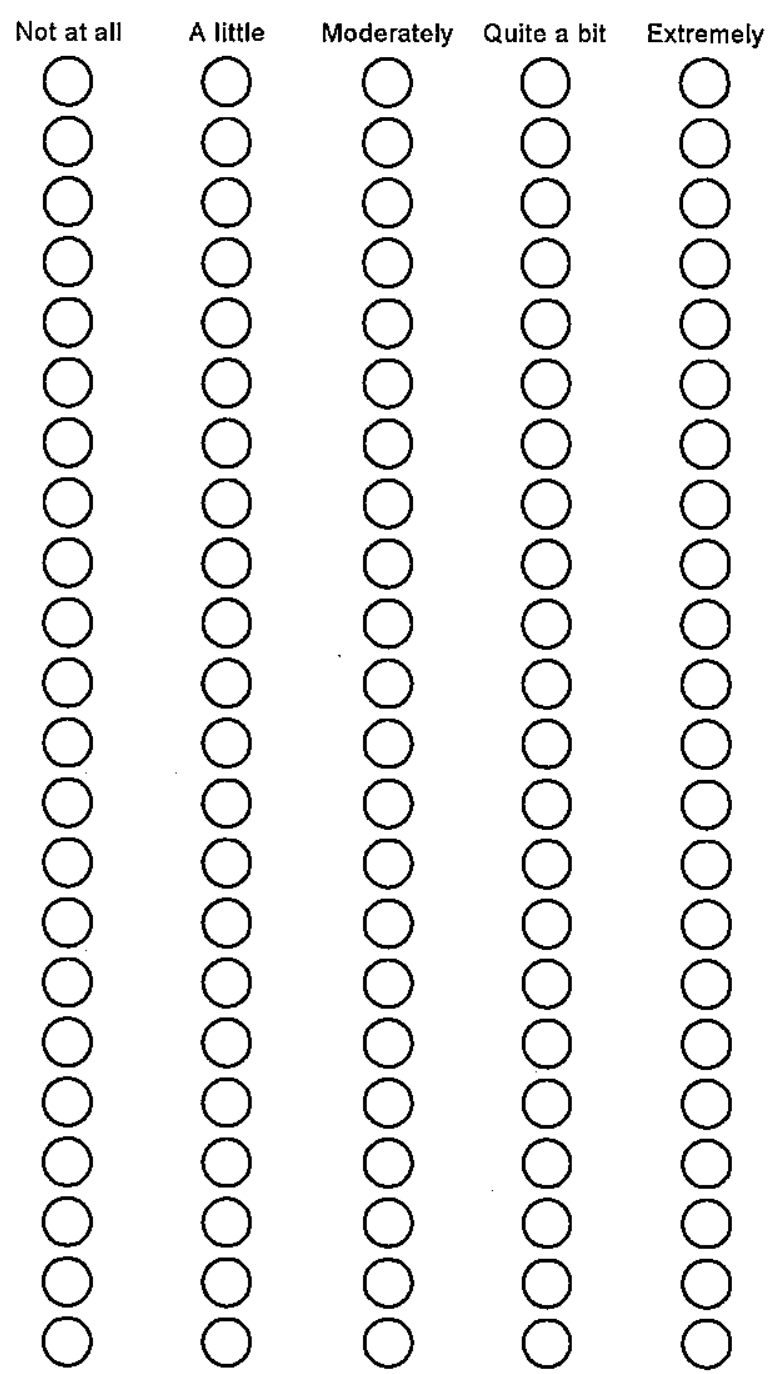




\section{Exploring the functions of non-suicidal self-injury}

\section{Section Six}

In this section, you will be asked to answer the following questions TWICE-once in relation to thoughts you may or may not have had BEFORE you injured yourself most recently and once in relation to thoughts you may or may not have had AFTER you injured yourself most recently.

Listed below are a variety of thoughts that pop into people's heads. Please read each thought and select YES if the thought occurred to you BEFORE your MOST RECENT episode of non-suicidal self-injury (i.e., the last time you hurt yourself on purpose without intending to kill yourself) or NO if the thought did not occur to you BEFORE your MOST RECENT episode of non-suicidal self-injury. Please select any or all that apply.

\section{BEFORE my MOST RECENT episode of non-suicidal self-injury, I}

\section{thought...}

I feel like l'm up against the world.

I'm no good.

I'm proud of myself.

Why can't I ever succeed?

No-one understands me.

I've let people down.

I feel fine.

I don't think I can go on.

I wish I were a better person.

No matter what happens, I know l'll make it.

I'm so weak.

My life's not going the way I want it to.

I can accomplish anything.

I'm so disappointed in myself.

Nothing feels good anymore.

1 feel good.

I can't stand this anymore.

I can't get started.

What's wrong with me?

l'm warm and comfortable.

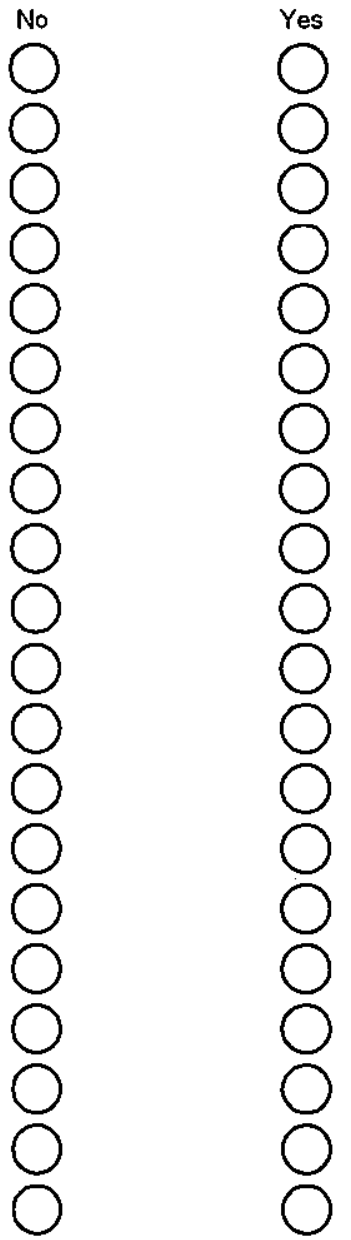


Exploring the functions of non-suicidal self-injury

2. Continued...

BEFORE my MOST RECENT episode of non-suicidal self-injury, I thought...

I wish I were somewhere else.

No

Yes

I can't get things together.

I hate myself.

I feel confident I can do anything I set my mind to.

l'm worthless.

Wish I could just disappear.

What's the matter with me?

I feel very happy.

I'm a loser.

My life is a mess.

l'm a failure.

This is cool.

I'll never make it.

I feel so helpless.

Something has to change.

There must be something wrong with me.

I'm luckier than most people.

My future is bleak.

It's just not worth it.

I can't finish anything.

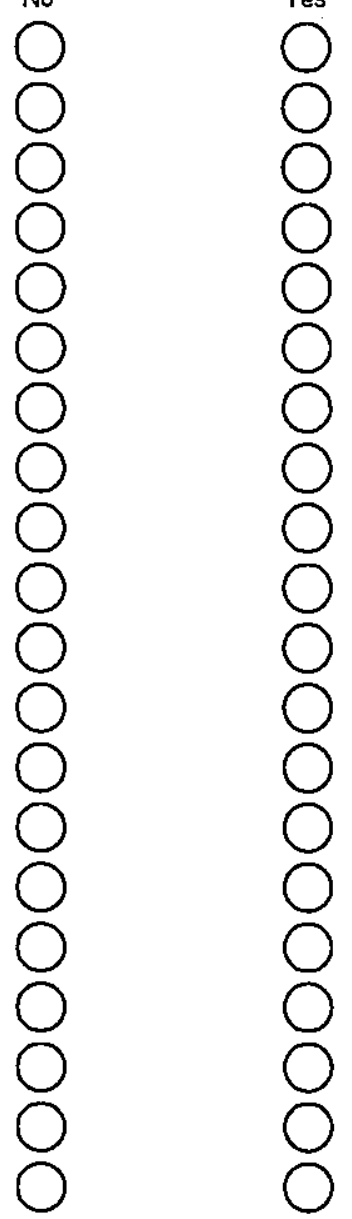




\title{
Exploring the functions of non-suicidal self-injury
}

Now please read each thought and select YES if the thought occurred to you AFTER your MOST RECENT episode of non-suicidal self-injury (i.e., the last time you hurt yourself on purpose without intending to kill yourself) or NO if the thought did not occur to you AFTER your MOST RECENT episode of non-suicidal self-injury. Please select any or all that apply.

\section{AFTER my MOST RECENT episode of non-suicidal self-injury, I} thought...

\author{
I feel like I'm up against the world. \\ I'm no good. \\ I'm proud of myself. \\ Why can't I ever succeed? \\ No-one understands me. \\ I've let people down. \\ I feel fine. \\ I don't think I can go on. \\ [ wish ] were a better person. \\ No matter what happens, I know I'll make it. \\ I'm so weak. \\ My life's not going the way / want it to. \\ I can accomplish anything. \\ I'm so disappointed in myself. \\ Nothing feels good anymore. \\ I feel good. \\ I can't stand this anymore. \\ I can't get started. \\ What's wrong with me? \\ I'm warm and comfortable.
}

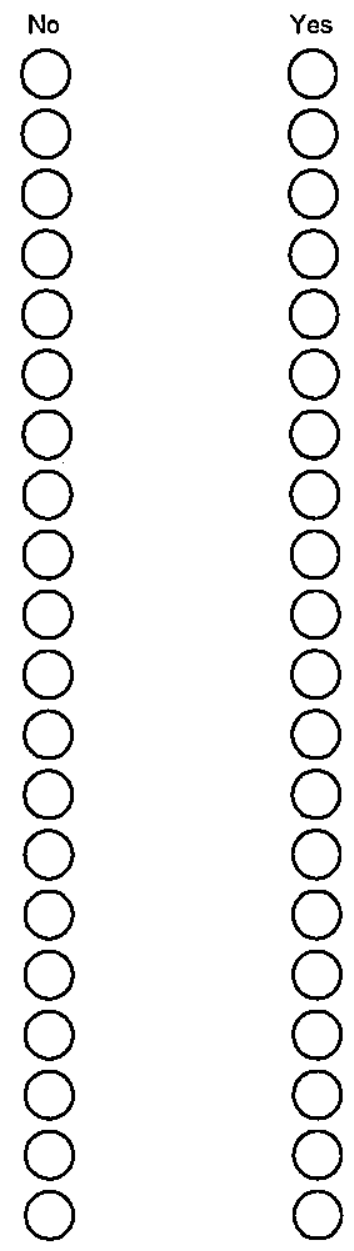




\section{Exploring the functions of non-suicidal self-injury}

\section{Continued...}

\section{AFTER my MOST RECENT episode of non-suicidal self-injury, I}

\section{thought...}

I wish I were somewhere else.

I can't get things together.

I hate myself.

I feel confident I can do anything I set my mind to.

l'm worthless.

Wish 1 could just disappear.

What's the matter with me?

I feel very happy.

I'm a loser.

My life is a mess.

I'm a failure.

This is cool.

I'll never make it.

I feel so helpless.

Something has to change.

There must be something wrong with me.

I'm luckier than most people.

My future is bleak.

It's just not worth it.

I can't finish anything.

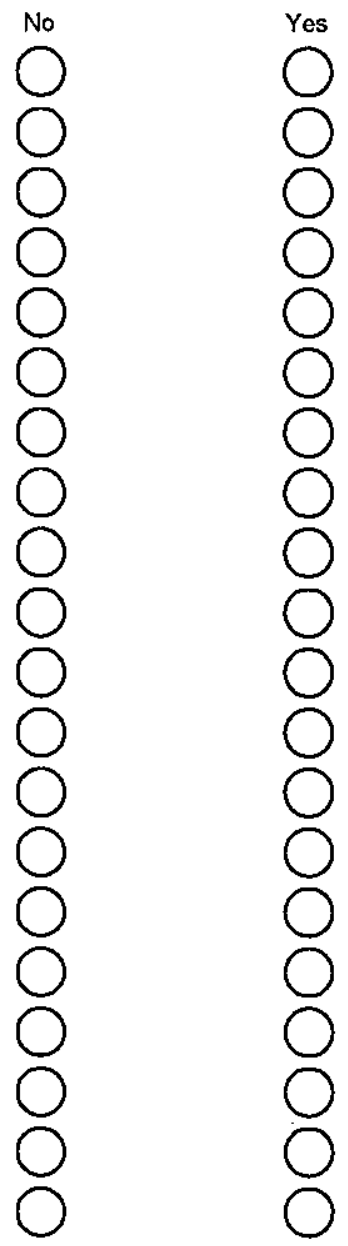




\section{Exploring the functions of non-suicidal self-injury}

\section{Section Seven}

Below is a list of statements that may or may not be relevant to your most recent experience of self-injury. Please select the option that indicates how relevant each statement was for you IN RELATION to your MOST RECENT episode of non-suicidal self-injury (i.e., the last time you hurt yourself on purpose without intending to kill yourself):

\section{1. 'When I self-injured, I was ...}

... calming myself down

... creating a boundary between myself and others

... punishing myself

... giving myself a way to care for myself (by attending to the wound)

... causing pain so 1 would stop feeling numb

... avoiding the impulse to attempt suicide

... doing something to generate excitement or exhilaration

... bonding with peers

... letting others know the extent of my emotional pain

... seeing if I could stand the pain

... creating a physical sign that I felt awful

... getting back at someone

... ensuring that I was self-sufficient

... releasing emotional pressure that had built up inside of me

... demonstrating that I was separate from other people

... expressing anger towards myself for being worthless or stupid

... creating a physical injury that was easier to care for than my emotional distress

... trying to feel something (as opposed to nothing) even if it was physical pain

... responding to suicidal thoughts without actually attempting suicide

... entertaining myself or others by doing something extreme
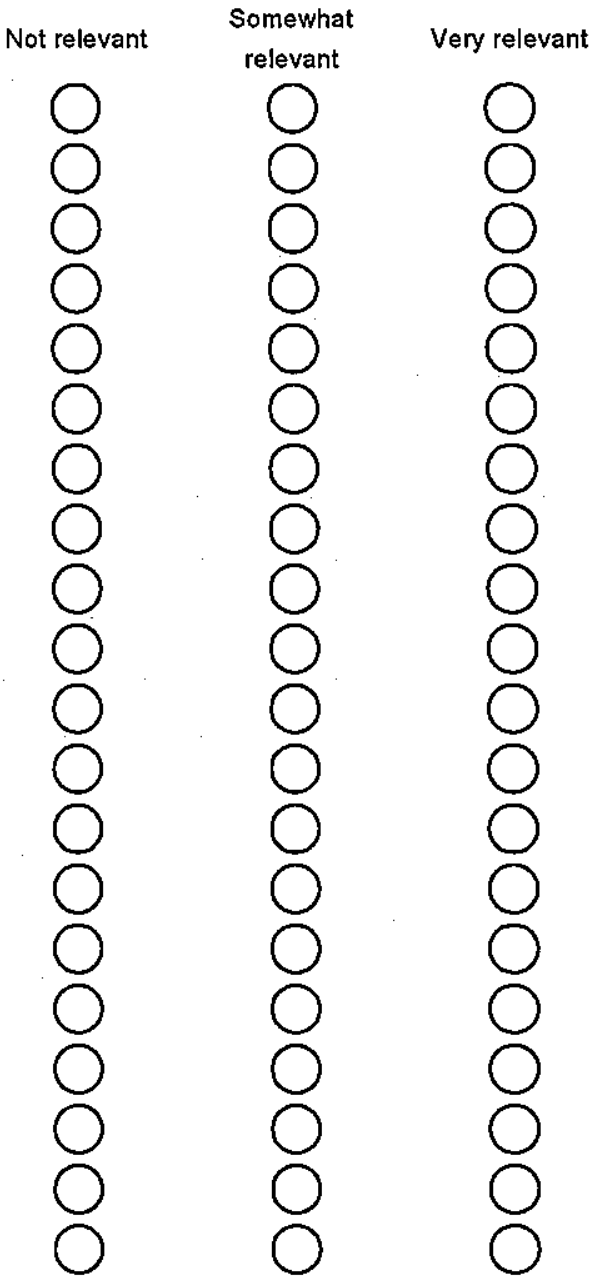


\section{Exploring the functions of non-suicidal self-injury}

\section{Continued...}

"When I self-injured, I was...

... fitting in with others

... seeking care or help from others

...demonstrating I was tough or strong

... proving to myself that my emotional pain was real

... getting revenge against others

... demonstrating that 1 did not need to rely on others for help

... reducing anxiety, frustration, anger, or other overwheiming emotions

... establishing a barrier between myself and others

... reacting to feeling unhappy with myself or disgusted with myself

... allowing myself to focus on treating the injury, which can be gratifying or satisfying

... making sure I was still alive when I didn't feel real

... putting a stop to suicidal thoughts

... pushing my limits in a manner akin to skydiving or other extreme activities

... creating a sign of friendship or kinship with friends or loved ones

... keeping a loved one from leaving or abandoning me

... proving I could take the physical pain

... signifying the emotional distress I was experiencing

... trying to hurt someone close to me

... establishing that I was autonomous/independent
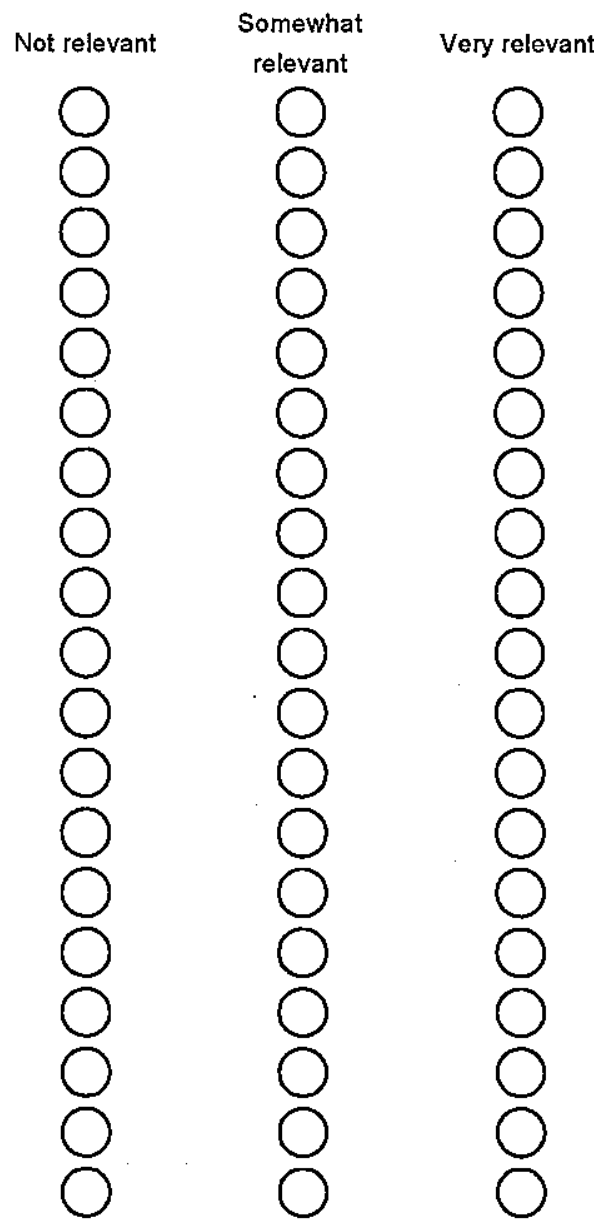


\section{Exploring the functions of non-suicidal self-injury}

9. A consequence I experienced following my most recent episode of non-sucidal selfinjury was:

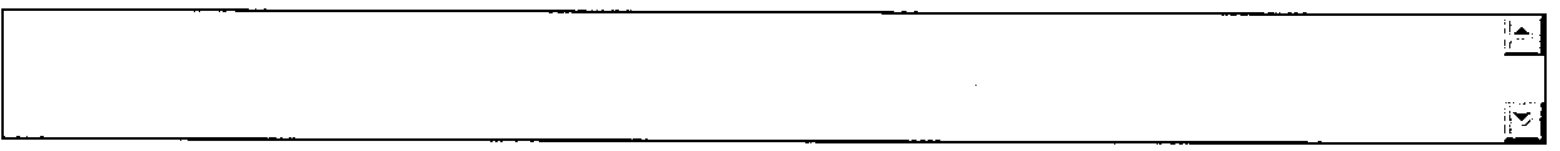

10. This consequence was...
Negative
Neutral
Positive 


\section{Exploring the functions of non-suicidal self-injury: Debriefing information}

Thank you for completing this survey. In this study, we were interested in the thoughts, feelings, and events that lead people to engage in non-suicidal self-injury. Researchers have identified that one of the main reasons why people injure themselves on purpose is to manage overwhelming, negative feelings such as sadness or anger. Other reasons people give for self-injury include: to punish themselves, to feel more in control, and to stop feeling numb.

Understanding why people self-injure is essential in order to effectively treat this behaviour. Very little research has been conducted in Aotearoa/New Zealand to explore non-suicidal self-injury. This research will help us to learn more about why people in Aotearoa/New Zealand injure themselves on purpose and, ultimately, may help us to identify the most effective treatments for non-suicidal self-injury.

Thank you once again for taking the time to participate in this study. If you know of anyone else who has injured themselves on purpose in the past 12 months, is at least 16 years of age, lives in Aotearoa/New Zealand, and you think they may be interested in completing this survey, please pass on the study details to them.

\section{Would you like to go into a draw to win one of two iPod Shuffies (2GB)?}

$\bigcirc$ Yes

$\bigcirc$ No

Would you like to receive a summary of the research results?

Yes

No

If you answered yes to either/both of the above questions, please enter your email address in the space provided:

Email Address:

Please note: Your contact details will not be linked to your survey answers. Your survey answers are anonymous. 


\section{Exploring the functions of non-suicidal self-injury: Debriefing information}

NEED TO TALK TO SOMEONE?

If you have found completing this survey distressing in any way, it may be helpful for you to talk to a friend, relative, or other support person (e.g., teacher, minister, counsellor) about how you are feeling. You could also call, email, or check out the websites of one of the following support organisations:

\section{Lifeline}

Free telephone counselling available 24 hours a day, 7 days a week Ph: (09) 5222999 (within Auckland); 0800543354 (outside Auckland) Website: http://www.lifeline.org.nz

Free email counselling

Email: chris@elifeline.co.nz

Website: http://www.elifeline.co.nz

\section{Youthline}

Free telephone counselling available 24 hours a day, 7 days a week

Ph: 0800376633

Website: http://www.youthline.co.nz

Email: talk@youthline.co.nz

Free TXT using your mobile phone to 234

\section{Warmline}

Warmline is a peer-run service, staffed by people who have all experienced mental illness in a real way. Warmline's service is free, and open to anyone who feels affected in some way by mental illness. Available $7.00 \mathrm{pm}-1.00 \mathrm{am}$, Tuesday-Sunday.

Ph: 0800200207

Website: http://www.wellink.org.nz/services/warmline.htm

\section{Rainbow Youth}

Rainbow Youth is an Auckland-based organisation providing support, information, advocacy and education for lesbian, gay, bisexual, transgender, intersex, fa'afafine, and takataapui young people (aged between 13 and 28 ) and their friends, family and whanau. Phone: (09) 3764155

Email: info@rainbowyouth.org.nz

Website: http://www.rainbowyouth.org.nz

\section{Alcohol Drug Association of New Zealand}

Helpline available 10am-10pm daily, 7 days a week

Ph: 0800787797

Website: http://www.alcoholdrughelp.org.nz 


\section{Coding scheme ${ }^{1}$}

"Unpleasant events" are defined as members of a set of events (e.g., death of a close relative, losing one's job), stimuli (e.g., strong electric shock), and behaviours (e.g., cleaning a mess) which are experienced by the individual to whom they occur as unpleasant, painful, noxious, or distressing.

1) First, code each response according to the following categories (responses may be given more than one code):

\begin{tabular}{|l|l|l|}
\hline Code & Category & Definition \\
\hline 01 & Health and well-being & $\begin{array}{l}\text { Events that involve injury, physical pain, and danger to the } \\
\text { self or to important others. These can be specific } \\
\text { psychopathological events (e.g., a binge) but do not include } \\
\text { ongoing mental health problems (e.g., bulimia). }\end{array}$ \\
\hline 02 & Achievement-Academic-Job & $\begin{array}{l}\text { Events that involve work, school, and other competitive } \\
\text { situations; achievement related failures, disappointments, } \\
\text { and difficulties. }\end{array}$ \\
\hline 03 & $\begin{array}{l}\text { Domestic, day-to-day } \\
\text { inconveniences }\end{array}$ & $\begin{array}{l}\text { Events that involve noise, crowding, minor physical } \\
\text { discomforts, mishaps, delays, and accidents. }\end{array}$ \\
\hline 04 & Interpersonal Relationships & $\begin{array}{l}\text { Events concerning rejection, separation, loss, and other } \\
\text { disappointing and painful interpersonal experiences. These } \\
\text { events can include memories of negative interpersonal } \\
\text { experiences. }\end{array}$ \\
\hline 05 & Legal & $\begin{array}{l}\text { Events involving encounters with the police, courts of law, } \\
\text { lawsuits, incarceration, and other legal problems. }\end{array}$ \\
\hline 06 & Material-Financial & Events involving financial losses and problems. \\
\hline 07 & Death Related & $\begin{array}{l}\text { The actual or remembered death of an important other or } \\
\text { events which remind the individual of the reality of death } \\
\text { (e.g., seeing a corpse). }\end{array}$ \\
\hline 08 & Other & $\begin{array}{l}\text { Events that could not be coded in any of the above } \\
\text { categories. }\end{array}$ \\
\hline 88 & No specific event & $\begin{array}{l}\text { General response that did not refer to a specific event } \\
\text { (e.g., I was feeling overwhelmed). }\end{array}$ \\
\hline Insufficient Information & $\begin{array}{l}\text { Response did not contain enough information about the } \\
\text { event to be coded. }\end{array}$ \\
\hline
\end{tabular}

2) Second, code each response again according to the following categories:

\begin{tabular}{|l|l|l|}
\hline Code & Category & Definition \\
\hline 13 & Self & $\begin{array}{l}\text { Self-related events that involve only the individual. These } \\
\text { can include memories of past events that involved others. }\end{array}$ \\
\hline 14 & Other & $\begin{array}{l}\text { Events which involve interacting with other people at the } \\
\text { time (i.e., not memories of past interactions). }\end{array}$ \\
\hline
\end{tabular}

\footnotetext{
${ }^{1}$ Adapted from the Unpleasant Events Schedule (Lewinsohn, Mermelstein, Alexander, \& MacPhillamy, 1983).
} 


\section{Debriefing information}

Thank you for completing this survey. In this study, we were interested in the thoughts, feelings, and events that lead people to engage in non-suicidal self-injury. Researchers have identified that one of the main reasons why people injure themselves on purpose is to manage overwhelming, negative feelings such as sadness or anger. Other reasons people give for self-injury include: to punish themselves, to feel more in control, and to stop feeling numb. Understanding why people self-injure is essential in order to effectively treat this behaviour. Very little research has been conducted in Aotearoa/New Zealand to explore non-suicidal self-injury. This research will help us to learn more about why people in Aotearoa/New Zealand injure themselves on purpose and, ultimately, may help us to identify the most effective treatments for non-suicidal selfinjury.

Thank you once again for taking the time to participate in this study. If you know of anyone else who has injured themselves on purpose in the past 12 months, is at least 16 years of age, lives in Aotearoa/New Zealand, and you think they may be interested in completing this survey, please pass on the study details to them.

\section{Need to talk to someone?}

If you have found completing this survey distressing in any way, it may be helpful for you to talk to a friend, relative, or other support person (e.g., teacher, minister, counsellor) about how you are feeling. You could also call, email, or check out the websites of one of the following support organisations:

\section{LifeLine}

Free telephone counselling available 24 hours a day, 7 days a week

Ph: (09) 5222999 (within Auckland)

0800543354 (outside Auckland)

Website: http://www.lifeline.org.nz

Free email counselling

Email: chris@elifeline.co.nz

Website: http://www.elifeline.co.nz

\section{Youthline}

Free telephone counselling available 24 hours a day, 7 days a week Ph: 0800376633

Website: http://www.youthline.co.nz

Email: talk@youthline.co.nz

Free TXT using your mobile phone to 234 


\section{Warmline}

Warmline is a peer-run service, staffed by people who have all experienced mental illness in a real way. Warmline's service is free, and open to anyone who feels affected in some way by mental illness. Available 7.00pm-1.00am, Tuesday-Sunday

Ph: 0800200207

Website: http://www.wellink.org.nz/services/warmline.htm

\section{Rainbow Youth}

Rainbow Youth is an Auckland-based organisation providing support, information, advocacy and education for lesbian, gay, bisexual, transgender, intersex, fa'afafine, and takataapui young people (aged between 13 and 28) and their friends, family and whanau. Phone: (09) 3764155

Email: info@rainbowyouth.org.nz

Website: http://www.rainbowyouth.org.nz

\section{Alcohol Drug Association of New Zealand}

Helpline available 10am-10pm daily, 7 days a week Ph: 0800787797

Website: http://www.alcoholdrughelp.org.nz 


\section{Emotions, Coping, and Non-suicidal Self-injury - Time 1}

\section{Information Sheet}

Principle Investigator:

Robyn Langlands, PhD Student

School of Psychology

PO Box 600

Victoria University of Wellington

Wellington

Email: robyn.langlands@vuw.ac.nz

Supervisor:

Dr Marc Wilson

School of Psychology

PO BOX 600

Victoria University of Wellington

Wellington

Email: marc.wilson@vuw.ac.nz

Ph: (04) 4635225

\section{Introduction}

You are invited to take part in a study designed to examine the relationships between emotions, coping strategies, and non-suicidal self-injury. Non-suicidal self-injury, also called deliberate self-harm, is hurting oneself on purpose without intending to kill oneself. The purpose of this research is to identify whether people with a history of non-suicidal selfinjury are more likely to experience negative emotions and thoughts than people who have never self-injured. We are also interested in examining whether people who have and have not self-injured use different coping strategies.

Who is conducting the research?

Robyn Langlands is conducting the study as part of her PhD thesls. Dr Marc Wilson is supervising the research.

\section{Who is being asked to participate in this study?}

At least 200 Psychology students are being invited to participate in this study. You are invited to participate in this study even if you have never self-injured. We are looking for two groups of people to take part: people who have a history of non-suicidal self-injury and people who have never injured themselves on purpose.

\section{What is involved if you agree to participate?}

If you agree to take part in this study, you will be asked to complete the same online survey at two different time points, two months apart. The survey contains questions about non-suicidal self-injury, coping strategies, emotions, and thoughts. We expect that each survey will take you no more than 30 minutes to complete. When you complete the first survey, you will be asked to enter your student ID and email address. In two months time, you will be sent an email containing a link to the same survey, which you will be asked to complete again. By completing the same survey twice, we will be able to identify whether your answers change over time. The survey will be hosted on a secure, password protected website and your answers will be completely confidential.

\section{What are the benefits?}

If you complete the first survey, you will receive 30 minutes worth of IPRP credits. If you complete the second survey, you will be sent a $\$ 10$ Motor Trade Assocation (MTA) voucher. Aside from these credits and the gift voucher, there are no guarantees that you will receive any other benefits from taking part in the study. However, the results of this research may be used to inform interventions for non-suicidal self-injury.

\section{Do I have to participate as part of my Psychology course requirements?}

No, your participation is entirely voluntary (your choice) and you do not have to take part in this study. There are a number of studies you can choose from in order to meet your course requirements. You are free to withdraw from the study at any time, without having to give a reason. 


\section{Emotions, Coping, and Non-suicidal Self-injury - Time 1}

\section{Are there any risks?}

Negative thoughts, emotions, and non-suicidal self-injury can be a very difficult topics to answer questions about and there is a risk that some of the questions asked may bring up past memories or feelings that are unpleasant or distressing. If appropriate, you may wish to ask a support person to be available while you complete the surveys. If you do become distressed while completing any of the questions, you can stop filling in the survey at any time. You do not have to answer all the questions. This study will not involve you receiving any form of psychological therapy. Every person who takes part in the study will be emailed a list of options for support. This list will also be available at the end of each survey. You are also welcome to contact Robyn Langlands or Dr Wilson if you have any concerns or questions about this study.

\section{What about confidentiality?}

No material that could personally identify you will be used in any reports on this study. We will keep your data for at least ten years after publication. You will never be identified in this research project or in any other presentation or publication. The information you provide will be coded by number only. In accordance with the requirements of some scientific journals and organisations, your coded data may be shared with other competent researchers. Your coded data may be used in other, related studies. A copy of the coded data will remain in the custody of Robyn Langlands and Dr Wilson.

What will happen at the end of the study?

You will be given the option of receiving a summary of the results once they have been analysed. There may be a delay between the end of the study and when the results are available.

Where can I get more information about the study?

If you have any questions about this study, please contact Robyn Langlands at robyn.langlands@vuw.ac.nz.

\section{What if I have concerns about the project?}

If you have any queries or concerns about your rights as a participant in this study, you may wish to contact an independent health and disability advocate:

Free phone: 0800555050

Free Fax: 08002 SUPPORT (0800 2787 7678)

Email: advocacy@hdc.org.nz

Statement of approval

This study has received ethical approval from the Multi-region Ethics Committee, ethics reference number (insert ethics reference number here).

\section{I hereby consent to participate in this study.}

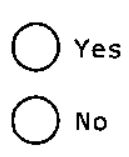

Investigating the relationships between emotions, coping, and non-suicidal self-injury, Version 1 


\section{Emotions, Coping, and Non-suicidal Self-injury - Time 1}

You did not consent to participate in this survey. Please close your browser window to exit. 


\section{Emotions, Coping, and Non-suicidal Self-injury - Time 1}

Please enter your student ID number and email address in the spaces provided below.

Student ID number:

Email address: 


\section{Emotions, Coping, and Non-suicidal Self-injury - Time 1}

\section{Section One}

In order to understand more about your responses to later parts of the survey, we would like to know a bit more about you.
Are you...
Oremale
Male
Other (Please specify)

\section{How old are you?}

Which ethnic group do you belong to? (Please select all that apply)

$\square$ New Zealand European

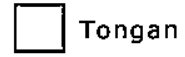

$\square$ Mãori

$\square$ Nivean

Samoan

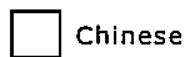

Cook Islands Maori Indian Other (Please state: e.g., Dutch, Japanese, Tokelauan)

Have you ever received a mental health diagnosis?

Yes

@o

If you answered YES, please select all that apply:

$\square$ Depression

Bipolar Disorder

Anorexia

Bulimia
Borderline Personality Disorder

Anxiety Disorder

Substance Use Disorder

Schizophrenia

Other (Please specify) 


\section{Emotions, Coping, and Non-suicidal Self-injury - Time 1}

Have you ever received therapy or counselling from a qualified practitioner (e.g., psychologist, counsellor)?

$\bigcirc$ Yes

No

If you answered YES, are you currently receiving therapy or counselling from a qualified practitioner (e.g., psychologist, counsellor)?

Ores

$\bigcirc N_{0}$ 


\section{Emotions, Coping, and Non-suicidal Self-injury - Time 1}

\section{Section Two}

The following section asks about a number of different things that people sometimes do to hurt themselves. Please be sure to read each question carefully and respond honestly. Often, people who do these kinds of things to themselves keep it a secret, for a variety of reasons. However, honest responses to these questions will provide us with greater understanding and knowledge about these behaviours and the best way to help people. Please answer yes to a question only if you did the behaviour intentionally, or on purpose, to hurt yourself. Do not respond yes if you did something accidentally (e.g., you tripped and banged your head on accident). Also, please be assured that your responses are completely confidential.

Have you ever intentionally (i.e., on purpose) cut your wrist, arms, or other area(s) of your body (without intending to kill yourself)? (Select one):

Yes

No

If YES, how many times have you done this? (If you can't remember, please estimate the number of times (e.g., 5, $10,100)$ you have done this)

\section{Have you ever intentionally (i.e., on purpose) burned yourself with a} cigarette, lighter, or a match? (Select one):

Yes

No

If YES, how many times have you done this? (If you can't remember, please estimate the number of times (e.g., 5 , 10,100 ) you have done this)

\section{Have you ever intentionally (i.e, on purpose) carved words, pictures, designs, or other marks into your skin? (Select one): \\ Yes \\ No}

If YES, how many times have you done this? (If you can't remember, please estimate the number of times (e.g., 5 , 10,100 ) you have done this) 


\section{Emotions, Coping, and Non-suicidal Self-injury - Time 1}

Have you ever intentionally (i.e., on purpose) severely scratched yourself, to the extent that scarring or bleeding occurred? (Select one):
Y Yes
No

If YES, how many times have you done this? (If you can't remember, please estimate the number of times (e.g., 5 , $10,100)$ you have done this)

Have you ever intentionally (i.e., on purpose) bitten yourself, to the extent that you broke the skin? (Select one):
Yes
No

If YES, how many times have you done this? (If you can't remember, please estimate the number of times (e.g., 5 , 10,100 ) you have done this)

Have you ever intentionally (i.e., on purpose) rubbed sandpaper on your body? (Select one):
Y Yes
No

If YES, how many times have you done this? (If you can't remember, please estimate the number of times (e.g., 5 , 10,100 ) you have done this)

Have you ever intentionally (i.e., on purpose) dripped acid onto your skin? (Select one):

Yes

No

If YES, how many times have you done this? (If you can't remember, please estimate the number of times (e.g., 5 , 10,100 ) you have done this) 


\section{Emotions, Coping, and Non-suicidal Self-injury - Time 1}

Have you ever intentionally (i.e., on purpose) used bleach or oven cleaner to scrub your skin? (Select one):

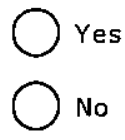

If YES, how many times have you done this? (If you can't remember, please estimate the number of times (e.g., 5 , 10,100 ) you have done this)

Have you ever intentionally ( $i_{n} e_{,}$, on purpose) stuck sharp objects such as needles, pins, staples, etc. into your skin, not including tattoos, ear piercing, needles used for drug use, or body piercing? (Select one):

Yes

$\bigcirc$ No

If YES, how many times have you done this? (If you can't remember, please estimate the number of times (e.g., 5 , 10,100 ) you have done this)

Have you ever intentionally (i.e., on purpose) rubbed glass into your skin? (Select one):

Y Yes

No

If YES, how many times have you done this? (If you can't remember, please estimate the number of times (e.g., 5 , 10,100 ) you have done this)

Have you ever intentionally (i.e., on purpose) broken your own bones? (Select one):

Yes

No

If YES, how many times have you done this? (If you can't remember, please estimate the number of times (e.g., 5 , $10,100)$ you have done this) 


\section{Emotions, Coping, and Non-suicidal Self-injury - Time 1}

Have you ever intentionally (i.e., on purpose) banged your head against something, to the extent that you caused a bruise to appear? (Select one):

Yres

No

If YES, how many times have you done this? (If you can't remember, please estimate the number of times (e.g., 5 , 10,100 ) you have done this)

Have you ever intentionally (i.e., on purpose) punched yourself, to the extent that you caused a bruise to appear? (Select one):

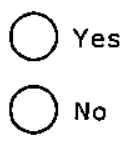

If YES, how many times have you done this? (If you can't remember, please estimate the number of times (e.g., 5 , 10,100 ) you have done this)

Have you ever intentionally (i.e., on purpose) prevented wounds from healing? (Select one):
Yes
No

If YES, how many times have you done this? (If you can't remember, please estimate the number of times (e.g., 5 , 10,100 ) you have done this)

Have you ever intentionally (i.e., on purpose) done anything else to hurt yourself that was not asked about in this questionnaire? (Select one):

Yes

No

If YES, what did you do to hurt yourself? How many times have you done this? (If you can't remember, please estimate the number of times (e.g., 5, 10, 100) you have done this)

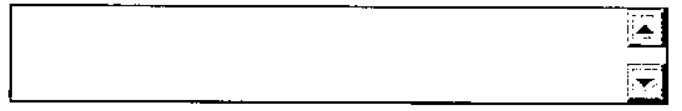

Did you answer YES to any of the questions on this page?
Yyes
No 


\section{Emotions, Coping, and Non-suicidal Self-injury - Time 1}

How old were you when you first hurt yourself on purpose without intending to kill yourself? (If you can't remember, please estimate how old you were)

Please estimate, using numbers, how many times you have hurt yourself on purpose without intending to kill yourself in the past two months.

When did you last hurt yourself on purpose without intending to kill yourself? (Select one)

Less than 1 week ago

More than 1 week but less than 1 month ago

More than 1 month but less than 6 months ago

More than 6 months but less than 1 year ago

More than 1 year ago

If you hurt yourself on purpose without intending to kill yourself more than 1 year ago, please indicate when this occurred: (Select one)

More than 1 year but less than 2 years ago

More than 2 years but less than 3 years ago

More than 3 years but less than 4 years ago

More than 4 years but less than 5 years ago

More than 5 years ago 


\section{Emotions, Coping, and Non-suicidal Self-injury - Time 1}

\section{Section Three}

Below you will find a list of statements. Please rate the truth of each statement as it applies to you. Use the following scale to make your choice.

I am able to take action on a problem even if I am uncertain what is the right thing to do.

I often catch myself daydreaming about things I've done and what I would do differently next time.

When I feel depressed or anxious, I am unable to take care of my responsibilities.

I rarely worry about getting my anxieties, worries, and feelings under control.

I'm not afraid of my feelings.

When I evaluate something negatively, I usually recognize that this is just a reaction, not an objective fact.

When I compare myself to other people, it seems that most of them are handling their lives better than I do.

Anxiety is bad.

If I could magically remove all the painful experiences I've had in my life, I would do so.

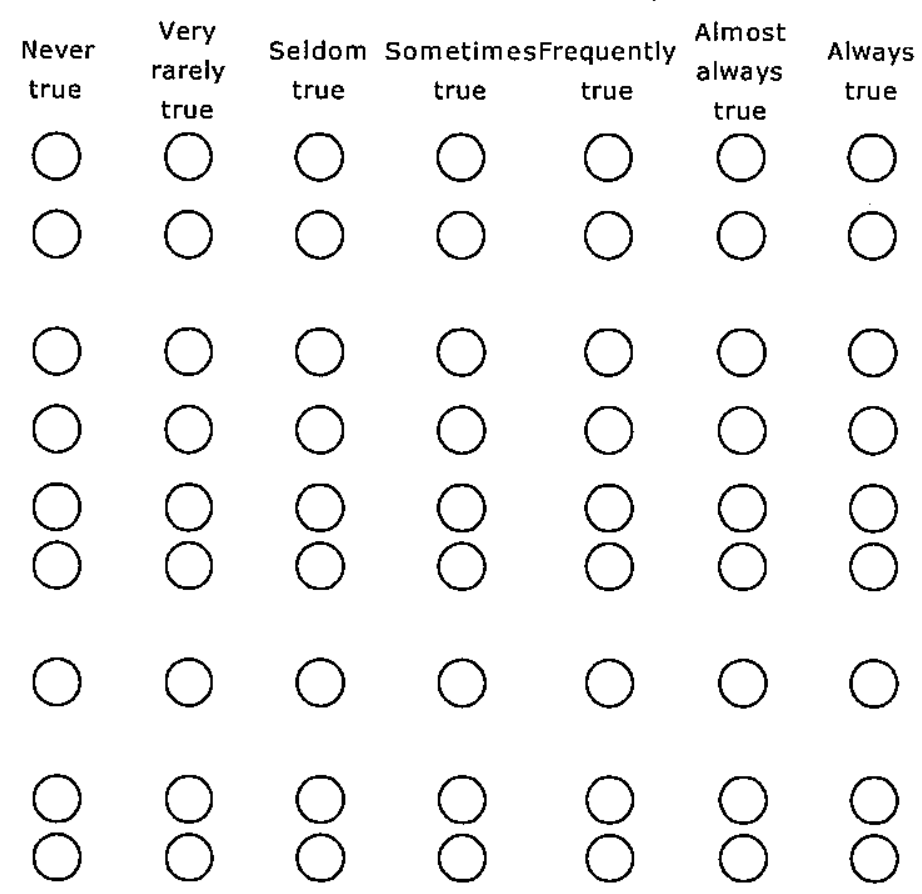




\section{Emotions, Coping, and Non-suicidal Self-injury - Time 1}

\section{Section Four}

This section consists of a number of words and phrases that describe different feelings and emotions. Read each item and then mark the appropriate answer in the space next to that word. Indicate to what extent you feel this way in general, that is, on the average. Use the following scale to record your answers.

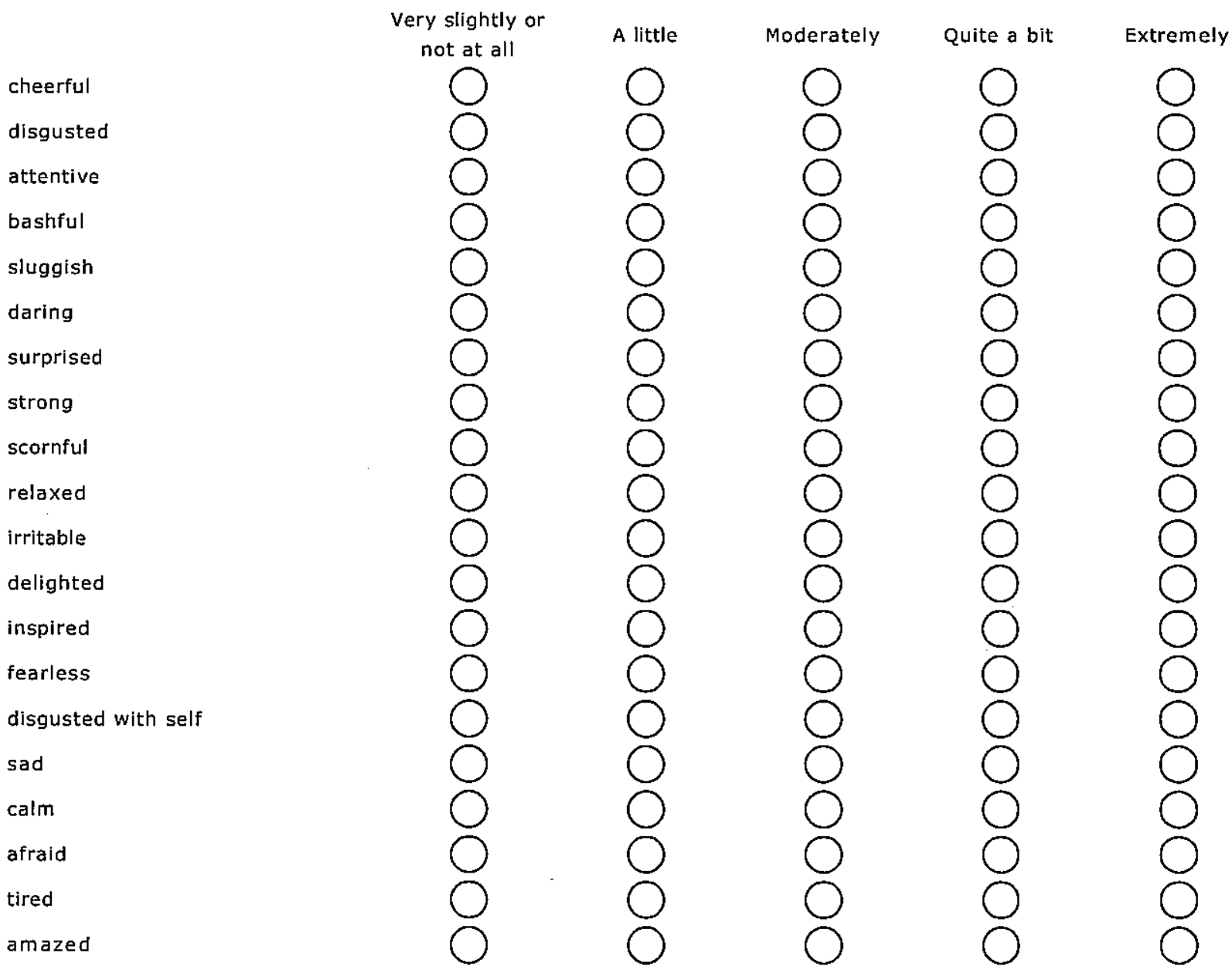




\section{Emotions, Coping, and Non-suicidal Self-injury - Time 1}

Continued...

$\begin{array}{lll}\text { shaky } & \text { happy } \\ \text { timid } \\ \text { alone } \\ \text { alert } \\ \text { upset } \\ \text { angry } \\ \text { bold } \\ \text { blue } \\ \text { shy } \\ \text { active } \\ \text { guilty } \\ \text { joyful } \\ \text { nervous } \\ \text { lonely } \\ \text { sleepy } \\ \text { excited } \\ \text { hostile } \\ \text { proud } \\ \text { jittery }\end{array}$




\section{Emotions, Coping, and Non-suicidal Self-injury - Time 1}

\section{Continued...}

lively
ashamed
at ease
scared
drowsy
angry at self
enthusiastic
downhearted
distressed
blameworthy
determined
frightened
astonished
interested
loathing
confissatisfied with self
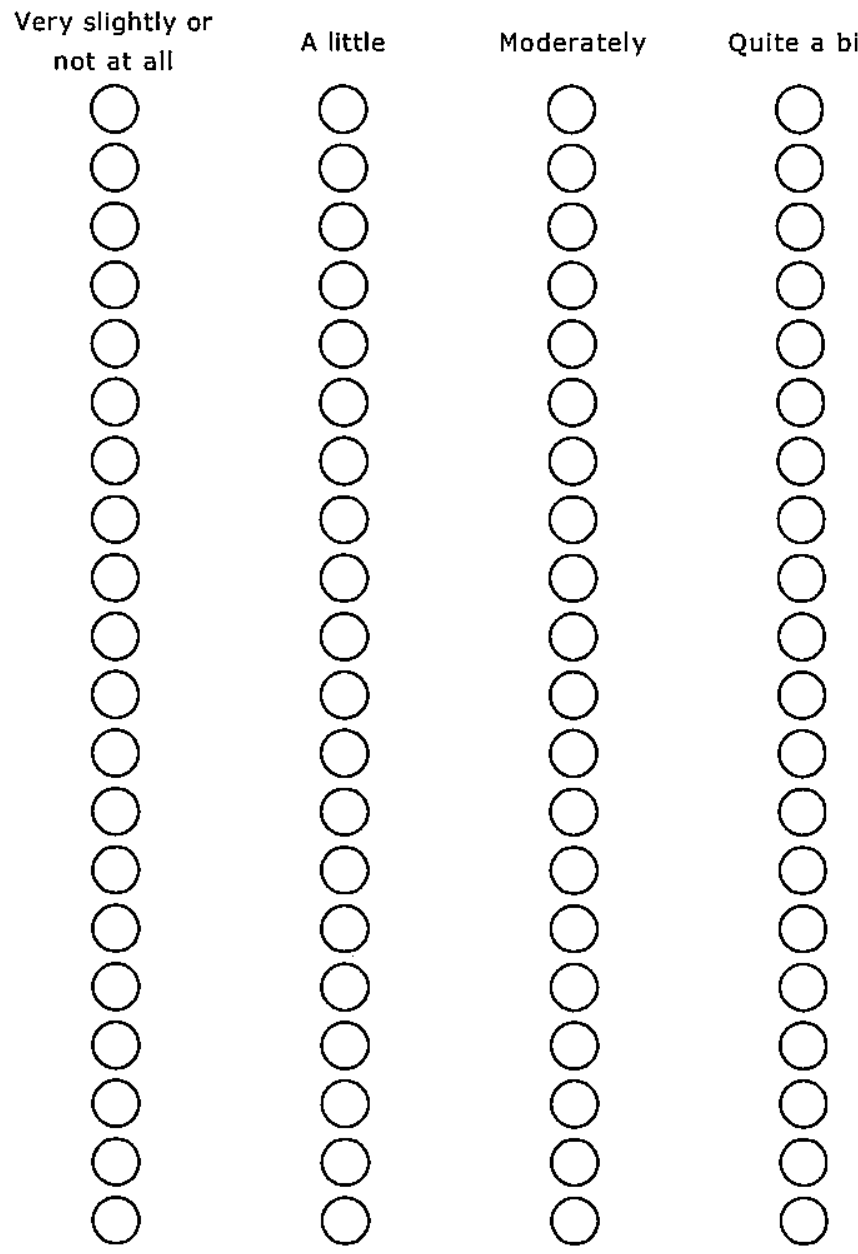

Extremely

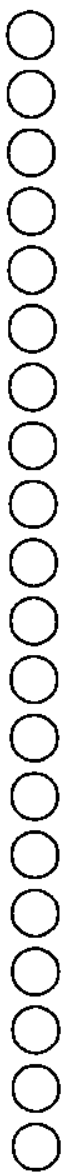




\section{Emotions, Coping, and Non-suicidal Self-injury - Time 1}

\section{Section Five}

This section is about thoughts. There are no right or wrong answers, so please respond honestly to each of the items below. Be sure to answer every item by selecting the appropriate response beside each.

There are things I prefer not to think about. Sometimes I wonder why I have the thoughts I do.
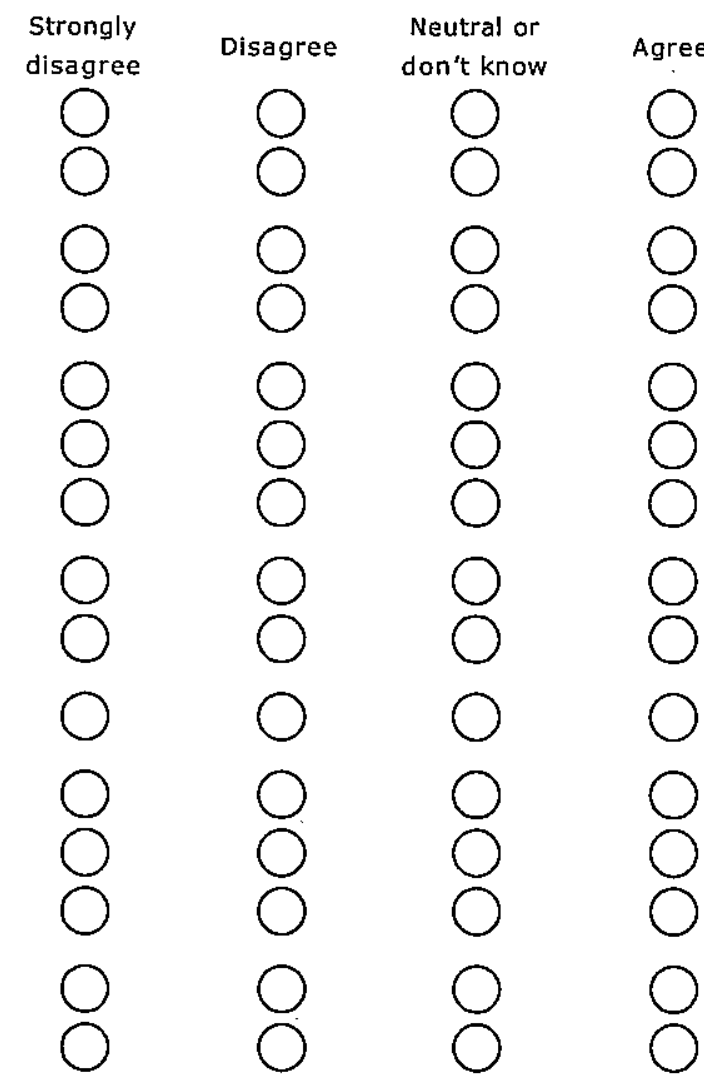

Strongly agree

I have thoughts that I cannot stop.

There are images that come to mind that I cannot erase.

My thoughts frequently return to one idea.

I wish I could stop thinking of certain things. Sometimes my mind races so fast I wish I could stop it.

I always try to put problems out of mind.

There are thoughts that keep jumping into my head.

Sometimes I stay busy just to keep thoughts from intruding on my mind.

There are things that I try not to think about.

Sometimes I really wish I could stop thinking. I often do things to distract myself from my thoughts.

I have thoughts that I try to avoid.

There are many thoughts that I have that I don't tell anyone.

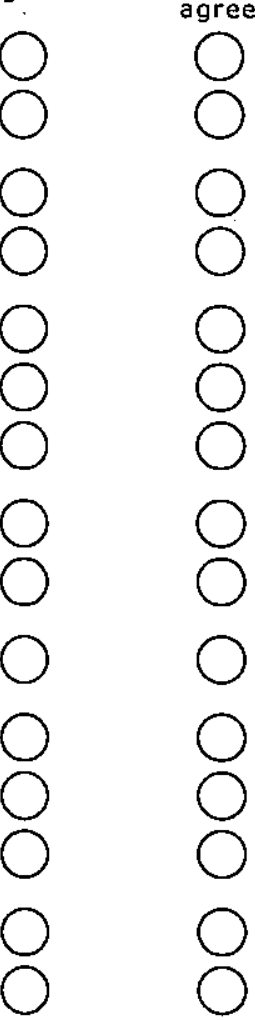




\section{Emotions, Coping, and Non-suicidal Self-injury - Time 1}

\section{Section Six}

Please read each statement and select the response which best indicates how much the statement applied to you over the past week. There are no right or wrong answers. Do not spend too much time on any statement. The rating scale is as follows:

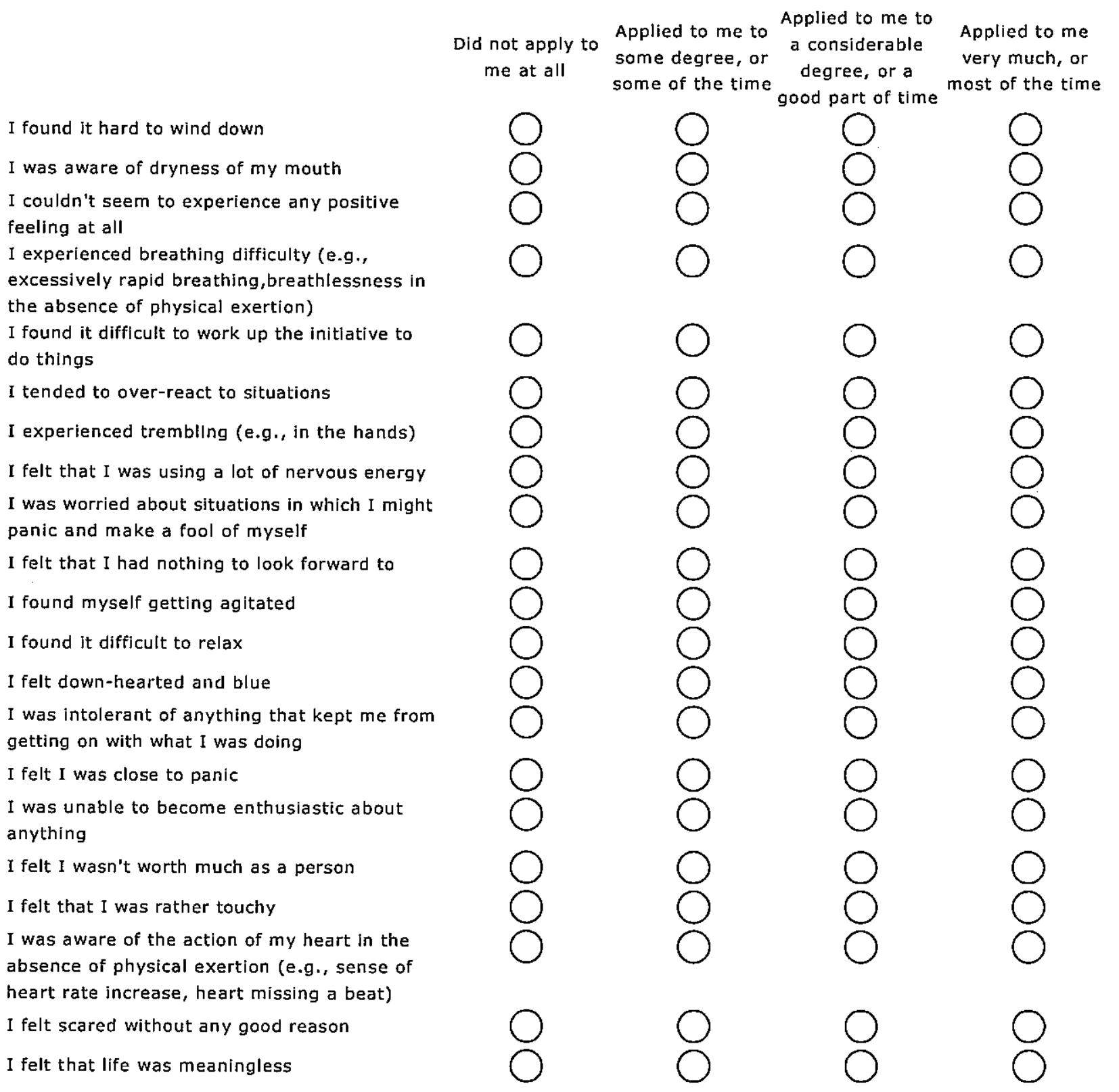




\section{Emotions, Coping, and Non-suicidal Self-injury - Time 1}

\section{Section Seven}

Listed below are a variety of thoughts that pop into people's heads. Please read each thought and indicate how frequently, if at all, the thought occurred to you over the last week. Please read each item carefully and select the appropriate response according to the following scale:

I'm no good.

I don't think I can go on.

I'm so disappointed in myself.

Nothing feels good anymore.

I can't stand this anymore.

I can't get started.

What's wrong with me?

I'm worthless.

I'll never make it.

I feel so helpless.

Something has to change.

There must be something wrong with me.

Not at all

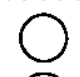

Sometimes Moderately often
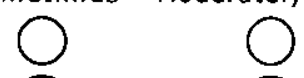

Often

All the time

My future is bleak.

It's just not worth it.

I can't finish anything.
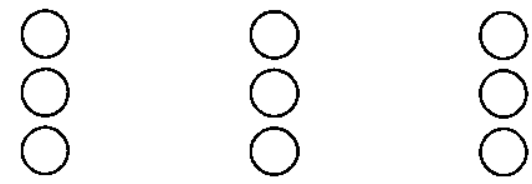


\section{Emotions, Coping, and Non-suicidal Self-injury - Time 1}

\section{Section Eight}

In this section, we are interested in how people respond when they confront difficult or stressful events in their lives. There are lots of ways to try to deal with stress. This questionnaire asks you to indicate what you generally do and feel when you experience stressful events. Obviously, different events bring out somewhat different responses, but think about what you usually do when you are under a lot of stress.

Then respond to each of the following items by selecting one response for each, using the choices listed just below. Please try to respond to each item separately in your mind from each other item. Choose your answers thoughtfully, and make your answers as true FOR YOU as you can. Please answer every item. There are no "right" or "wrong" answers, so choose the most accurate answer for YOU-not what you think "most people" would say or do. Indicate what YOU usually do when YOU experience a stressful event.

I turn to work or other activities to take my mind off things.

I concentrate my efforts on doing something about the situation I'm in.

I say to myself "this isn't real."

I use alcohol or other drugs to make myself feel better.

I get emotional support from others.

I give up trying to deal with it.

I take action to try to make the situation better.

I refuse to believe that it has happened. I say things to let my unpleasant feelings escape.

I get help and advice from other people. I use alcohol or other drugs to help me get through it.

I try to see it in a different light, to make it seem more positive.

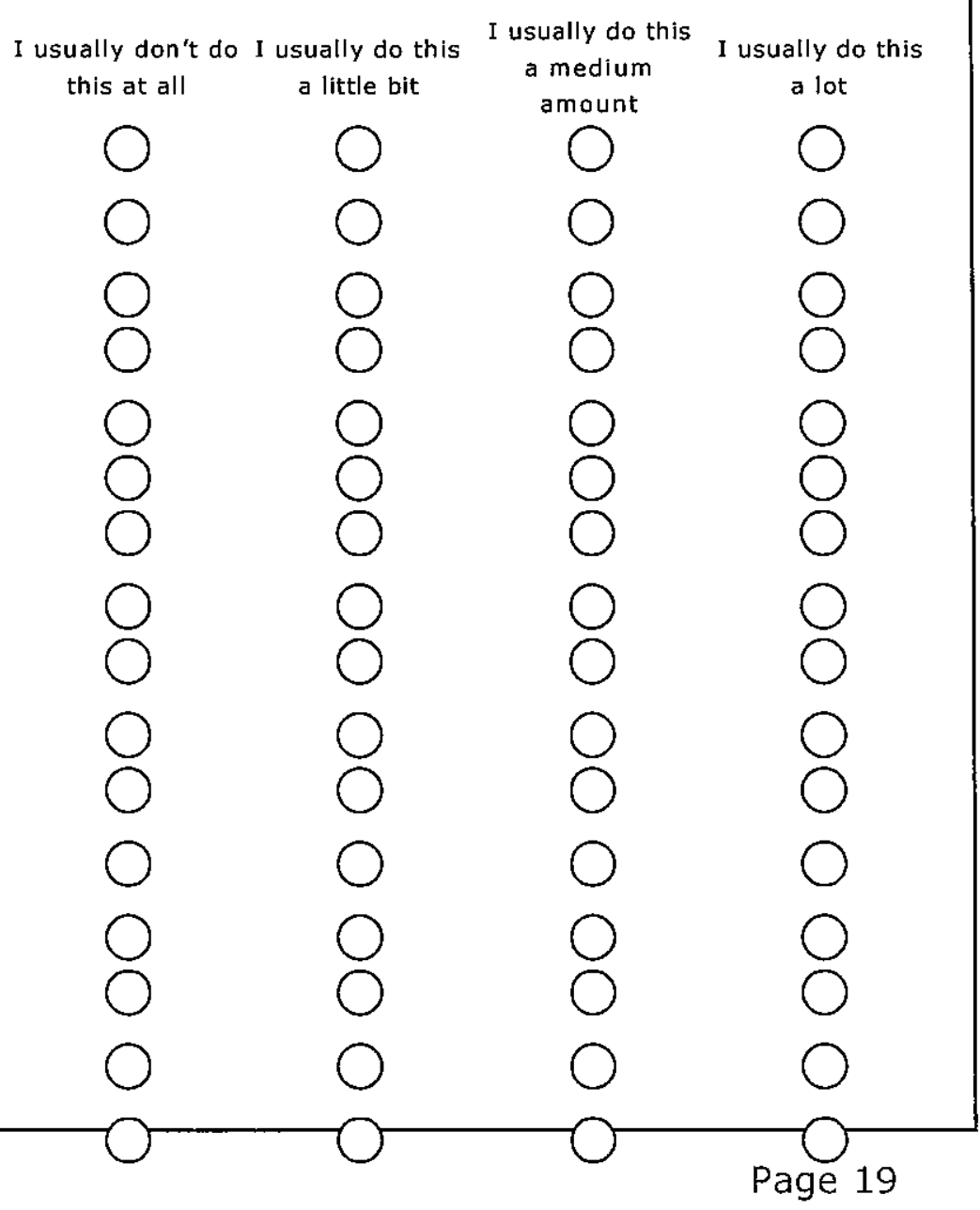

criticise myself.

I try to come up with a strategy about what to do.

I get comfort and understanding from someone. 


\section{Emotions, Coping, and Non-suicidal Self-injury - Time 1}

I give up the attempt to cope.

I look for something good in what is

happening.

I make jokes about it.

I do something to think about it less, such as going to movies, watching TV, reading, daydreaming, sleeping, or shopping.

I accept the reality of the fact that it has happened.

0

I try to find comfort in my religion or spiritual beliefs.

I try to get advice or help from other people about what to do.

I learn to live with it.

I think hard about what steps to take.

I blame myself for things that happened.

I pray or meditate.

I make fun of the situation.
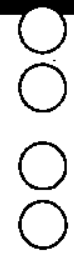

$\bigcirc$

$\bigcirc$<smiles>O</smiles><smiles>O</smiles>

0<smiles>O</smiles> 


\section{Emotions, Coping, and Non-suicidal Self-injury - Time 1}

\section{Options for Support}

Thank you very much for your time. It is much appreciated. You will be emailed a link to the second survey in two months time.

\section{Do you need support?}

If you are feeling distressed or upset, it may be helpful for you to talk to a friend, relative, or other support person (e.g., teacher, minister, counsellor) about how you are feeling. Alternatively, you could contact one of the following.

\section{In crisis?}

If you need urgent mental health support, please phone 111 or one of the following Crisis and Assessment Treatment Teams (CATT):

- If you live in the greater Wellington region (i.e., Wellington, Porirua, or Kapiti) phone 4949169.

. If you live in the Hutt Valley, phone 5666999 and ask for the CAT team.

\section{Need to talk to someone?}

If you need to talk to someone, please contact one of the following:

- Lifeline 0800543354

- Depression Helpline 0800111757

- Youthline 0800376633

- Warmline 0800200207

Need a referral or counselling?

Angelique $O^{\prime}$ Connell is a registered Clinical Psychologist who works in the Victoria Psychology Clinic, located on the 5th floor of the Easterfield Building. Phone 4636400 or email: psychclinic@vuw.ac.nz to book an appointment.

Victoria University has a free, confidential Counselling Service for students. Phone 4635310 or email: counsellingservice@vuw.ac.nz to book an appointment. 



\section{Emotions, Coping, and Non-suicidal Self-injury - Time 2}

Thank you for taking the time to participate in the second survey.

Please type your student ID number and email address in the spaces provided below.

\section{Student ID number:}

\section{Email address:}




\section{Emotions, Coping, and Non-suicidal Self-injury - Time 2}

\section{Section One}

The following section asks about a number of different things that people sometimes do to hurt themselves. Please be sure to read each question carefully and respond honestly. Often, people who do these kinds of things to themselves keep it a secret, for a variety of reasons. However, honest responses to these questions will provide us with greater understanding and knowledge about these behaviours and the best way to help people. Please answer yes to a question only if you did the behaviour intentionally, or on purpose, to hurt yourself. Do not respond yes if you did something accidentally (e.g., you tripped and banged your head on accident). Also, please be assured that your responses are completely confidential.

Have you ever intentionally (i.e, on purpose) cut your wrist, arms, or other area(s) of your body (without intending to kill yourself)? (Select one):

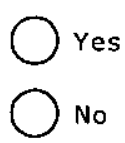

If YES, how many times have you done this? (If you can't remember, please estimate the number of times (e.g., 5 , 10,100 ) you have done this)

\section{Have you ever intentionally (i.e., on purpose) burned yourself with a cigarette, lighter, or a match? (Select one):

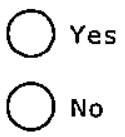

If YES, how many times have you done this? (If you can't remember, please estimate the number of times (e.g., 5 , 10,100 ) you have done this)

\section{Have you ever intentionally (i.e., on purpose) carved words, pictures, designs, or other marks into your skin? (Select one): \\ Yes \\ No}

If YES, how many times have you done this? (If you can't remember, please estimate the number of times (e.g., 5 , $10,100)$ you have done this) 
Emotions, Coping, and Non-suicidal Self-injury - Time 2

Have you ever intentionally (i.e., on purpose) severely scratched yourself, to the extent that scarring or bleeding occurred? (Select one):

Oyes
No

If YES, how many times have you done this? (If you can't remember, please estimate the number of times (e.g., 5, 10,100 ) you have done this)

Have you ever intentionally (i.e., on purpose) bitten yourself, to the extent that you broke the skin? (Select one):

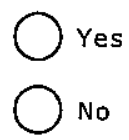

If YES, how many times have you done this? (If you can't remember, please estimate the number of times (e.g., 5 , $10,100)$ you have done this)

Have you ever intentionally (i.e., on purpose) rubbed sandpaper on your body? (Select one):

Yes

No

If YES, how many times have you done this? (If you can't remember, please estimate the number of times (e.g., 5 , 10,100 ) you have done this)

Have you ever intentionally (i.e., on purpose) dripped acid onto your skin? (Select one):

Yes

No

If YES, how many times have you done this? (If you can't remember, please estimate the number of times (e.g., 5 , 10,100 ) you have done this) 


\section{Emotions, Coping, and Non-suicidal Self-injury - Time 2}

Have you ever intentionally (i.e., on purpose) used bleach or oven cleaner to scrub your skin? (Select one):

OYes
No

If YES, how many times have you done this? (If you can't remember, please estimate the number of times (e.g., 5 , $10,100)$ you have done this)

Have you ever intentionally (i.e., on purpose) stuck sharp objects such as needles, pins, staples, etc. into your skin, not including tattoos, ear piercing, needles used for drug use, or body piercing? (Select one):
Y Yes
No

If YES, how many times have you done this? (If you can't remember, please estimate the number of times (e.g., 5, $10,100)$ you have done this)

Have you ever intentionally (i.e., on purpose) rubbed glass into your skin? (Select one):

Yes

No

If YES, how many times have you done this? (If you can't remember, please estimate the number of times (e.g., 5 , 10,100 ) you have done this)

Have you ever intentionally (i.e., on purpose) broken your own bones? (Select one):

$\bigcirc$ Yes

No

If YES, how many times have you done this? (If you can't remember, please estimate the number of times (e.g., 5, $10,100)$ you have done this) 


\section{Emotions, Coping, and Non-suicidal Self-injury - Time 2}

Have you ever intentionally (i.e., on purpose) banged your head against something, to the extent that you caused a bruise to appear? (Select one):

$\bigcirc$ Yes

ONo

If YES, how many times have you done this? (If you can't remember, please estimate the number of times (e.g., 5, 10,100 ) you have done this)

Have you ever intentionally (i.e., on purpose) punched yourself, to the extent that you caused a bruise to appear? (Select one):

OYes

No

If YES, how many times have you done this? (If you can't remember, please estimate the number of times (e.g., 5, 10,100 ) you have done this)

Have you ever intentionally (i.e., on purpose) prevented wounds from healing? (Select one):

Yes

No

If YES, how many times have you done this? (If you can't remember, please estimate the number of times (e.g., 5 , 10,100 ) you have done this)

Have you ever intentionally (i.e., on purpose) done anything else to hurt yourself that was not asked about in this questionnaire? (Select one):

Ores

No

If YES, what did you do to hurt yourself? How many times have you done this? (If you can't remember, please estimate the number of times (e.g., 5, 10, 100) you have done this)

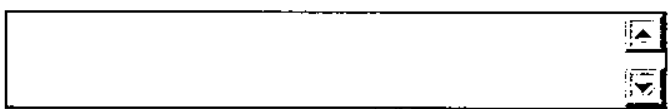

Did you answer YES to any of the questions on this page?

$\bigcirc$ Yes

No 


\section{Emotions, Coping, and Non-suicidal Self-injury - Time 2}

How old were you when you first hurt yourself on purpose without intending to kill yourself? (If you can't remember, please estimate how old you were)

Please estimate, using numbers, how many times you have hurt yourself on purpose without intending to kill yourself in the past two months.

When did you last hurt yourself on purpose without intending to kill yourself? (Select one)

Less than 1 week ago

More than 1 week but less than 1 month ago

More than 1 month but less than 6 months ago

More than 6 months but less than 1 year ago

More than 1 year ago

If you hurt yourself on purpose without intending to kill yourself more than 1 year ago, please indicate when this occurred: (Select one)

More than 1 year but less than 2 years ago

More than 2 years but less than 3 years ago

More than 3 years but less than 4 years ago

More than 4 years but less than 5 years ago

More than 5 years ago 


\section{Emotions, Coping, and Non-suicidal Self-injury - Time 2}

\section{Section Two}

Below you will find a list of statements. Please rate the truth of each statement as it applies to you. Use the following scale to make your choice.

am able to take action on a problem even if $\mathrm{I}$
$\mathrm{m}$ uncertain what is the right thing to do.
often catch myself daydreaming about things
ve done and what I would do differently next
Whe.
wen I feel depressed or anxious, I am unable
rarely worry about getting my anxieties, worries,
'm feelings under control.
When I evaluate something negatively, I usually
ecognize that this is just a reaction, not an
bjective fact.
When I compare myself to other people, it
eems that most of them are handling their lives
etter than I do.
Anxiety is bad.
f I could magically remove all the painful
xperiences I've had in my life, I would do so.


Emotions, Coping, and Non-suicidal Self-injury - Time 2

\section{Section Three}

This section consists of a number of words and phrases that describe different feelings and emotions. Read each item and then mark the appropriate answer in the space next to that word. Indicate to what extent you feel this way in general, that is, on the average. Use the following scale to record your answers.

\begin{tabular}{|c|c|c|c|c|c|}
\hline & $\begin{array}{l}\text { Very slightly or } \\
\text { not at all }\end{array}$ & A little & Moderately & Quite a bit & Extremely \\
\hline cheerful & & & & ) & \\
\hline disgusted & & & & $J$ & \\
\hline attentive & & & & D & \\
\hline bashful & & & & D & \\
\hline sluggish & & & & & \\
\hline daring & & & & ) & \\
\hline surprised & & & ) & D & \\
\hline strong & & & ) & 0 & \\
\hline scornful & & & & $D$ & \\
\hline relaxed & & & & & \\
\hline irritable & & & & 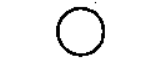 & \\
\hline delighted & & & ) & D & \\
\hline inspired & & & ) & D & \\
\hline fearless & & & & 5 & \\
\hline disgusted with self & & & & & \\
\hline sad & & & ) & & \\
\hline calm & & & & & \\
\hline afraid & & & & & \\
\hline tired & & & & & \\
\hline amazed & & & & & \\
\hline
\end{tabular}




\section{Emotions, Coping, and Non-suicidal Self-injury - Time 2}

\section{Continued...}

shaky
happy
timid
alone
alert
upset
angry
bold
blue
shy
active
guilty
joyful
nervous
lonely
sleepy
excited
hostile
proud
jittery

Very slightly or

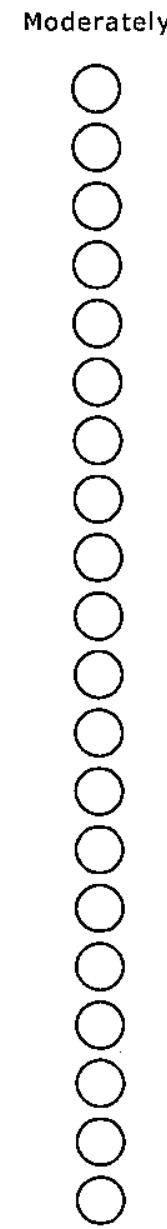

Quite a bit

Extremely

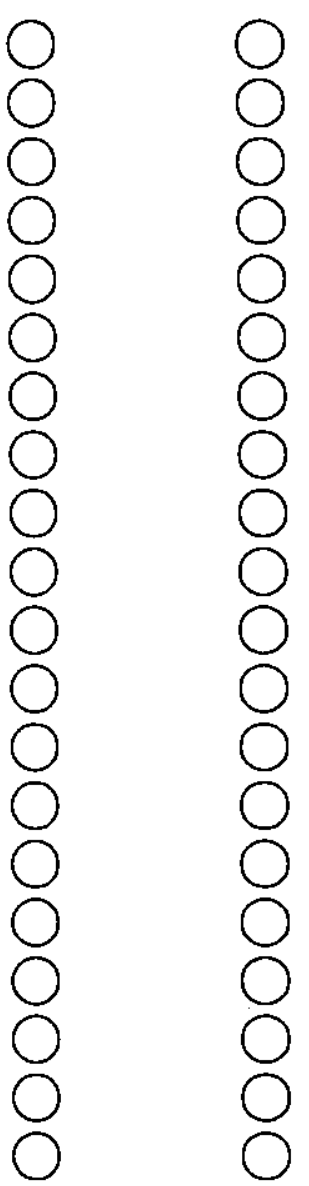




\section{Emotions, Coping, and Non-suicidal Self-injury - Time 2}

Continued...

lively

ashamed

at ease

scared

drowsy

angry at self

enthusiastic

downhearted

sheepish

distressed

blameworthy

determined

frightened

astonished

interested

loathing

confident

energetic

concentrating

dissatisfied with self
Very slightly or not at all

0

0

0

0

0

0

0

0

0

0

0

0

0

0

0

0

0

0

0

0
A little

0

0

0

0

0

$\bigcirc$

0

$\bigcirc$

0

0

0

0

0

0

0

0

0

0

0

$\bigcirc$
Moderately

0

0

0

0

$\bigcirc$

0

0

0

0

0

0

0

0

0

0

0

0

0

$\bigcirc$
Quite a bit

Extremely

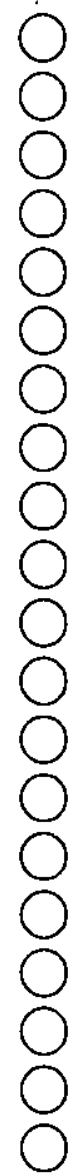

0<smiles></smiles><smiles>c1ccccc1</smiles><smiles></smiles><smiles>O</smiles><smiles></smiles> 


\section{Emotions, Coping, and Non-suicidal Self-injury - Time 2}

\section{Section Four}

This section is about thoughts. There are no right or wrong answers, so please respond honestly to each of the items below. Be sure to answer every item by selecting the appropriate response beside each.

There are things I prefer not to think about.
Sometimes I wonder why I have the thoughts I
do.
I have thoughts that I cannot stop.
There are images that come to mind that I
cannot erase.
My thoughts frequently return to one idea.
I wish I could stop thinking of certain things.
Sometimes my mind races so fast I wish I
could stop it.
I always try to put problems out of mind.
There are thoughts that keep jumping into my
head.
Sometimes I stay busy just to keep thoughts
Them intruding on my mind.
Sometimes I really wish I could stop thinking.
I often do things to distract myself from my
thoughts.
I have thoughts that I try to avoid.
There are many thoughts that I have that I
don't tell anyone.




\section{Emotions, Coping, and Non-suicidal Self-injury - Time 2}

\section{Section Five}

Please read each statement and select the response which best indicates how much the statement applied to you over the past week. There are no right or wrong answers. Do not spend too much time on any statement. The rating scale is as follows:

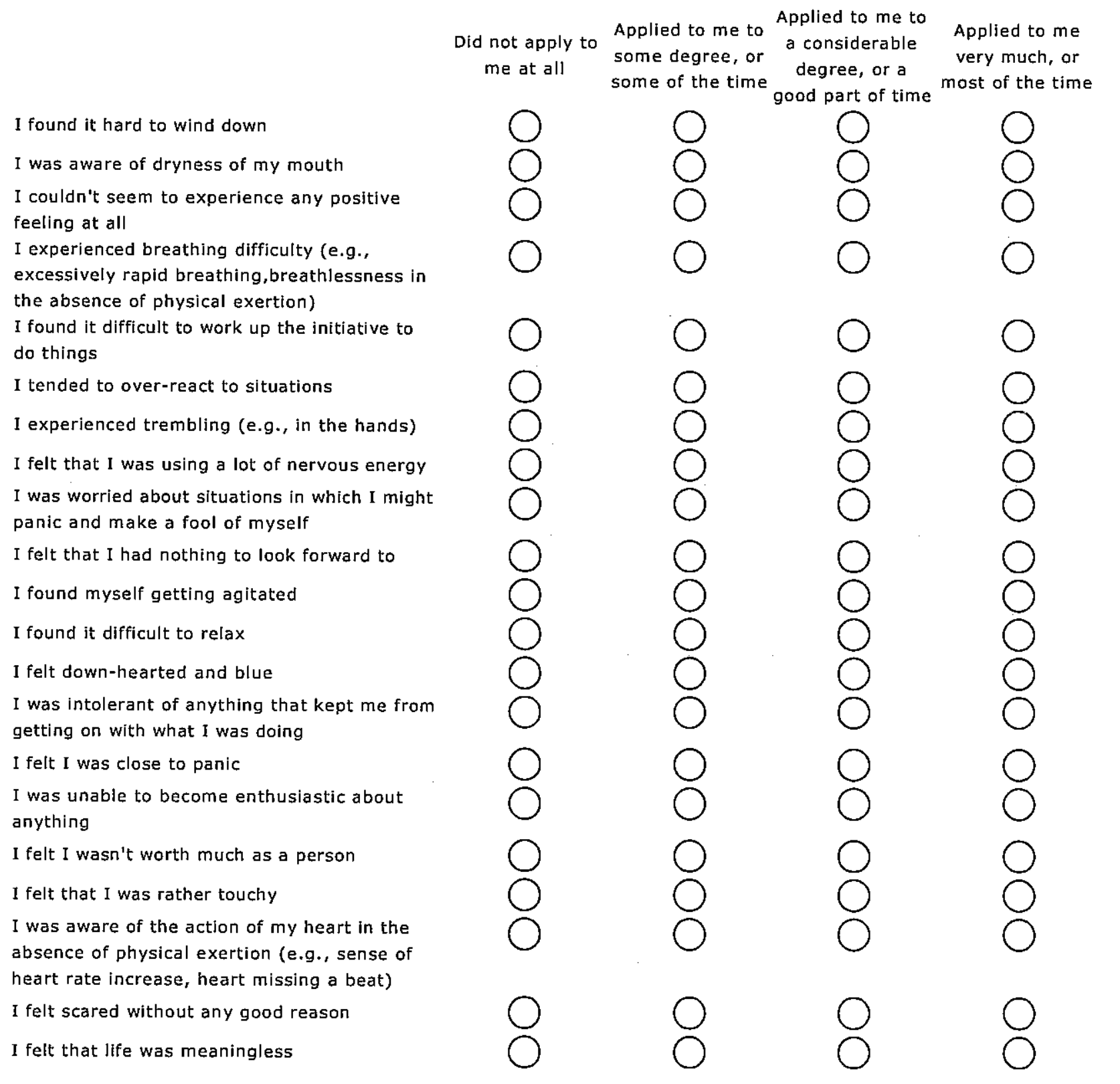




\section{Emotions, Coping, and Non-suicidal Self-injury - Time 2}

\section{Section Six}

Listed below are a variety of thoughts that pop into people's heads. Please read each thought and indicate how frequently, if at all, the thought occurred to you over the last week. Please read each item carefully and select the appropriate response according to the following scale:

I'm no good.

I don't think I can go on.

I'm so disappointed in myself. Nothing feels good anymore.

I can't stand this anymore.

1 can't get started.

What's wrong with me?

I'm worthless.

I'll never make it.

I feel so helpless.

Something has to change.

There must be something wrong with me.

Not at all

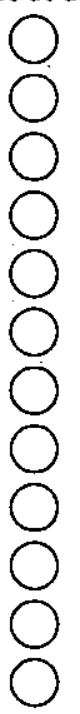

My future is bleak.

It's just not worth it.

I can't finish anything.
Sometimes Moderately often
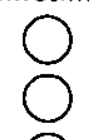

$\bigcirc$

0

$\bigcirc$

$\bigcirc$

O

O

0

$\bigcirc$

0

$\bigcirc$

0
All the time

Often

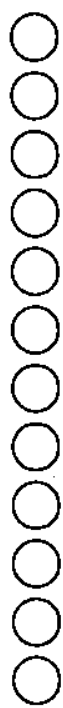

0
0
0
0
0
0
0
0
0
0
0
0 


\section{Emotions, Coping, and Non-suicidal Self-injury - Time 2}

\section{Section Seven}

In this section, we are interested in how people respond when they confront difficult or stressful events in their lives. There are lots of ways to try to deal with stress. This questionnaire asks you to indicate what you generally do and feel when you experience stressful events. Obviously, different events bring out somewhat different responses, but think about what you usually do when you are under a lot of stress.

Then respond to each of the following items by selecting one response for each, using the choices listed just below. Please try to respond to each item separately in your mind from each other item. Choose your answers thoughtfully, and make your answers as true FOR YOU as you can. Please answer every item. There are no "right" or "wrong" answers, so choose the most accurate answer for YOU-not what you think "most people" would say or do. Indicate what YOU usually do when YOU experience a stressful event.

I turn to work or other activities to take my mind off things.

I concentrate my efforts on doing something about the situation I'm in.

I say to myself "this isn't real."

I use alcohol or other drugs to make myself feel better.

I get emotional support from others.

I give up trying to deal with it.

I take action to try to make the situation better.

I refuse to believe that it has happened. I say things to let my unpleasant feelings escape.

I get help and advice from other people. I use alcohol or other drugs to help me get through it.

I try to see it in a different light, to make it seem more positive.

I usually don't do I usually do this this at all

$\bigcirc$

I criticise myself.

I try to come up with a strategy about what to do.

I get comfort and understanding from someone.

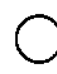

0

$\bigcirc$

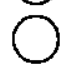

O

$\bigcirc$

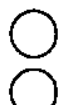

$\bigcirc$

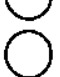

0

I usually do this I usually do this a medium a lot amount

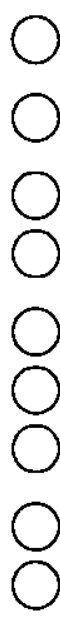

O

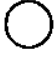

O

0

O

$\bigcirc$

$\bigcirc$ 


\section{Emotions, Coping, and Non-suicidal Self-injury - Time 2}
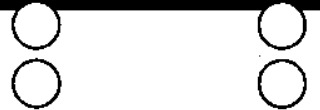

$\bigcirc$

I give up the attempt to cope.

I look for something good in what is

happening.

I make jokes about it.

I do something to think about it less, such as going to movies, watching TV, reading,

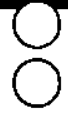
daydreaming, sleeping, or shopping.

I accept the reality of the fact that it has happened.

I express my negative feelings.

I try to find comfort in my religion or spiritual beliefs.

I try to get advice or help from other people about what to do.

I learn to live with it.

I think hard about what steps to take.

I blame myself for things that happened.

I pray or meditate.

I make fun of the situation. $\bigcirc$

$\bigcirc$

0

0

0

0

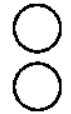

0

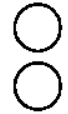

0

0

0

0

0

()

0

0

0

0

0 


\section{Emotions, Coping, and Non-suicidal Self-injury - Time 2}

\section{Debriefing Information}

Thank you for completing this study. Your time and contribution to this research is much appreciated. In this study, we were interested in how people with and without a history of non-suicidal self-injury experience emotions and how they cope with stress.

A recent theoretical model of non-suicidal self-injury called the Experiential Avoidance Model (Chapman, Gratz, \& Brown, 2006) proposes that when people with a history of non-suicidal self-injury experience stressful events that trigger negative, distressing emotions, they will feel the urge to escape from those emotions. As a result, they will use particular strategies to deal with their distress such as trying not to think about the event, drinking or using substances, or hurting themselves on purpose. In this study, we wanted to test this model to determine whether it is a valid explanation for why people injure themselves on purpose. If it is valid, then it can be used to inform clinical interventions for self-injurious behaviours.

\section{Would you like to receive a summary of the research results?}

Y Yes

No

\section{Please type your postal address in the space provided so we can send you a \$10 MTA voucher for completing this survey:}

\section{Do you need support?}

If you are feeling distressed or upset, it may be helpful for you to talk to a friend, relative, or other support person (e.g., teacher, minister, counsellor) about how you are feeling. Alternatively, you could contact one of the following.

In crisis?

If you need urgent mental health support, please phone 111 or one of the following Crisis and Assessment Treatment Teams (CATT):

- If you live in the greater Wellington region (i.e., Wellington, Porirua, or Kapiti) phone 4949169.

- If you live in the Hutt Valley, phone 5666999 and ask for the CAT team.

\section{Need to talk to someone?}

If you need to talk to someone, please contact one of the following:

- Lifeline 0800543354

- Depression Helpline 0800111757

- Youthline 0800376633

- Warmline 0800200207

Need a referral or counselling?

Angelique $O^{\prime} C o n n e l l$ is a registered Clinical Psychologist who works in the Victoria Psychology Clinic, located on the 5th floor of the Easterfield Building. Phone 4636400 or email: psychelinic@vuw.ac.nz to book an appointment.

Victoria University has a free, confidential Counselling Service for students. Phone 4635310 or email: counsellingservice@vuw.ac.nz to book an appointment. 


\section{Options for Support}

\section{Do you need support?}

If you are feeling distressed or upset, it may be helpful for you to talk to a friend, relative, or other support person (e.g., teacher, minister, counsellor) about how you are feeling. Alternatively, you could contact one of the following.

\section{In crisis?}

If you need urgent mental health support, please phone 111 or one of the following Crisis and Assessment Treatment Teams (CATT):

- If you live in the greater Wellington region (i.e., Wellington, Porirua, or Kapiti) phone 4949169.

- If you live in the Hutt Valley, phone 5666999 and ask for the CAT team.

\section{Need to talk to someone?}

If you need to talk to someone, please contact one of the following:

- Lifeline 0800543354

- Depression Helpline 0800111757

- Youthline 0800376633

- Warmline 0800200207

\section{Need a referral or counselling?}

Angelique O'Connell is a registered Clinical Psychologist who works in the Victoria Psychology Clinic, located on the 5th floor of the Easterfield Building. Phone 4636400 or email: psychclinic@vuw.ac.nz to book an appointment.

Victoria University has a free, confidential Counselling Service for students. Phone 4635310 or email: counselling-service@vuw.ac.nz to book an appointment. 